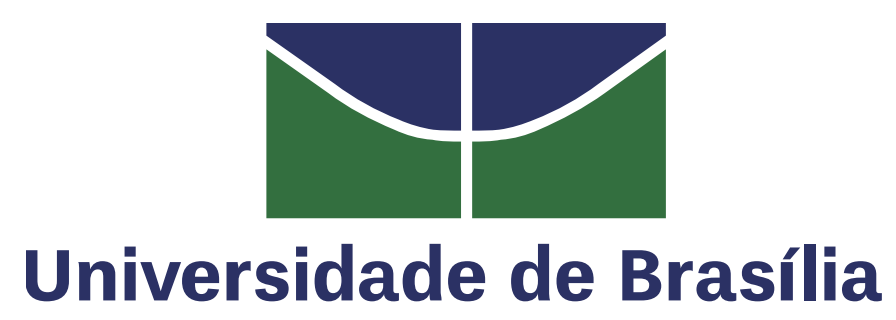

INSTITUTO DE GEOCIÊNCIAS

Pós-gRaduação em Geologia

\title{
ANÁLISE ESTRATIGRÁFICA EM ALTA RESOLUÇÃO: \\ EXEMPLO EM RAMPA CARBONÁTICA DOMINADA POR \\ MICROBIALITOS dA FORMAÇÃo SALITRE, BACIA dO \\ IRECÊ, BAHIA.
}

Ana Virgínia Alves de Santana

TESE DE Doutorado $n^{\circ} 130$

Orientador: Prof. Dr. Farid Chemale Júnior

BRASÍLIA, DF 


\title{
$\Psi$ \\ Universidade de Brasília
}

INSTITUTO DE GEOCIÊNCIAS

PóS-GRAdUAÇÃo EM GEOLOGIA

\section{ANÁLISE ESTRATIGRÁFICA EM ALTA RESOLUÇÃO:}

\section{EXEMPLO EM RAMPA CARBONÁTICA DOMINADA POR}

MICROBIALITOS dA FORMAÇÃo SALITRE, BACIA dO

\author{
IRECÊ, BAHIA.
}

TESE DE DOUTORADO

ANA VIRGÍNIA ALVES DE SANTANA

TESE DE DOUTORADO ELABORADA JUNTO AO Programa de Pós-GRADUAÇÃo em GEologia (ÁREA dE CONCENTRAÇÃo GEOLOGIA REGIONAL), DO INSTITUTO DE GEOCIÊNCIAS (IG) DA UNIVERSIDADE DE BRASÍLIA (UNB) PARA obTENÇÃo do TítULO DE DOUTORA EM GEOLOGIA.

\footnotetext{
Orientador: Professor Dr. Farid Chemale JúNior

EXAMINADORES:

Professor Dr. Aroldo Misi (UFBA)

Professor Dr. Carlos Jose Souza de Alvarenga (UnB)

Professor Dr. José Eloi Guimarães Campos (UnB)

Professora Dra. Marly Babinski (USP)
}

BRASÍLIA, DF 
À minha família espiritual, universal. À minha família terrena - às rochas, em especial. Com amor, respeito e gratidão.

“Uma educação pela pedra: por lições; para aprender da pedra, frequentá-la; captar sua voz inenfática, impessoal (pela de dicção ela começa as aulas).

(...) lições da pedra (de fora para dentro, cartilha muda), para quem soletrá-la." João Cabral de Melo Neto 


\section{AGRADECIMENTOS}

Concluo a presente pesquisa e sinto sincera gratidão!

\section{Meus guias espirituais. AMOR, AMOR, AMOR!}

* Minha família terrena. Suporte e amor!

* Meu querido orientador, Farid Chemale Júnior, por me "pegar nos braços"...E pelos cabelos! Você é especial!

* Antônio Jorge Magalhães, Cícero Paixão Pereira, Claiton Scherer, Ednilson Bento Freire, Felipe Guadagnin e Guilherme Raja Gabaglia Carinho e incientivo constantes!

* Membros da banca.

* Meus amigos do Cerrado - meu canto de dormir e sonhar! - e do meu outro país, RS - canto meu, da minha alma. E também aqueles da minha saudosa Bahia, canto da delícia e do gozo.

* Aos, à época, estudantes de graduação UnB, UFRGS, UFBA: Ana Spohr, Andrei Vechini, Hemeli Ligabue, Hugo Vidal, Marina Duarte, Ricardo Maahs, Tiago Morais e Viviane Araújo - ajuda fundamental!

* Zelo durante os trabalhos de campo: Henrique Lima, Luan Krug, Staff da Fora da Trilha, Faz. Rio Preto, Lapa Doce, Pratinha, Torrinhas. E toda comunidade local.

* Pesquisadores e administradores da Rede Temática de Caracterização e Modelagem de Reservatórios (CARMOD) da PETROBRAS.

* Geólogos dos cursos de campo da Petrobras na Chapada Diamantina, especialmente Daniel Galvão, Júlio Sanglard e Rodrigo Bunevich, pela solicitude sempre demonstrada.

* Programa de Pós-graduação em Geologia da UnB - Secretaria, professores e colegas. Especialmente à coordenadora Adalene Silva, por toda ajuda, e pós-graduando Rafael Santana, pelo auxílio em campo.

* Programa de Pós-graduação em Geologia da Universidade Federal do Rio Grande do Sul - Secretaria, professores e colegas. Principalmente aos que auxiliaram nos trabalhos de campo e discussões geológicas: Carlos Feijó, pós-graduandos Eduardo Espíndola, Ezequiel Souza, João Formolo Ferronatto, e professores De Ros, Evandro Lima e Karin Goldenberg.

* Ministério do Meio Ambiente - MMA e Instituto Chico Mendes de Conservação da Biodiversidade - ICMBio.

* Conselho Nacional de Desenvolvimento Científico e Tecnológico (CNPq).

* Governo do Brasil - Pelo Ensino laico, gratuito e de qualidade. 


\section{CAMINHOS DO CORAÇÃO}

\section{Gonzaguinha}

Há muito tempo que eu saí de casa

Há muito tempo que eu caí na estrada

Há muito tempo que eu estou na vida

Foi assim que eu quis, e assim eu sou feliz

Principalmente por poder voltar

A todos os lugares aonde já cheguei

Pois lá deixei um prato de comida

Um abraço amigo, um canto prá dormir e sonhar

E aprendi que se depende sempre

De tanta, muita, diferente gente

Toda pessoa sempre é as marcas

Das lições diárias de outras tantas pessoas

E é tão bonito quando a gente entende

Que a gente é tanta gente onde quer que a gente vá

E é tão bonito quando a gente sente

Que nunca está sozinho por mais que pense estar

É tão bonito quando a gente pisa firme

Nessas linhas que estão nas palmas de nossas mãos

É tão bonito quando a gente vai à vida

Nos caminhos onde bate bem mais forte o coração. 


\section{Resumo}

Santana, A.V.A. Análise estratigráfica em alta resolução: exemplo em rampa carbonática dominada por microbialitos da Formação Salitre, Bacia do Irecê, Bahia. Tese (Doutorado em Geologia) - Instituto de Geociências, Universidade de Brasília, Brasília, 2016.

A Formação Salitre registra a sequência carbonática neoproterozoica que recobre o cráton do São Francisco na região central da Bahia, nordeste do Brasil. O presente estudo objetivou fornecer um inédito arcabouço de estratigrafia de sequências em alta resolução para o trecho superior da Unidade B, sul do sinclinal de Irecê, na bacia homônima. Dez fácies e oito subfácies foram caracterizadas. A sucessão vertical de fácies mostra diferentes associações faciológicas: rampa intermediária com influência microbial (FA1); rampa interna rasa/rampa intermediária proximal (FA2); rampa interna dominada por microbialitos (FA3); rampa subaérea/sabkha (FA4). Estas FAs compõem um típico sistema carbonático marinho desenvolvido em uma rampa homoclinal. A análise sequencial em alta resolução distinguiu sequências elementares e de pequena escala em intervalos com a predominância das FA3 e FA4. Sequências elementares possuem espessura de algumas dezenas de centímetros. Estas sequências foram delineadas em campo a partir de uma série de fatores ordenados que pela sucessão vertical demonstrasse uma periodicidade/variabilidade no tipo do crescimento microbial. Sequências de pequena escala são constituídas a partir das sequências elementares. Sua demarcação ocorre a partir de critérios sedimentológicos e com o auxílio de perfis gama espectral. Sequências de média escala foram delineadas a partir de mudanças paleoambientais, como mudanças de associações faciológicas. Uma sequência de ampla escala, dezenas de metros de espessura, sugere a mais significativa mudança nas condições ambientais. Análises isotópicas de $\mathrm{C} / \mathrm{O}$ foram realizadas em amostras da porção basal da unidade $B$. Valores pouco negativos de $\delta^{13} C$, próximos de $0.0 \%$, sugerem uma assinatura isotópica típica de oceano. Análises de $\mathrm{U} / \mathrm{Pb}$ em SHRIMP, foram realizadas em siltito tufáceo (tufito) e em rocha com significativa contribuição epiclástica. A idade de deposição máxima da Formação Salitre é restringida pela idade do zircão mais novo, próxima a $670 \mathrm{Ma}$, obtida na amostra de tufito. As regiões fonte possíveis para os zircões da Formação Salitre, de acordo com as idades geocronológicas publicadas são (1) Faixa Riacho do Pontal, relacionada ao magmatismo distal em ambiente tectônico de margem passiva; (2) Faixa AraçuaíCongo Oeste, relacionada ao processo extensional de grande escala, bem documentado na contraparte africana da paleoplaca São Francisco-Congo (vulcanismo La Loiula); e (3) Faixa Brasília, relacionado com os eventos orogênicos. As idades mais antigas dos zircões detríticos são provenientes principalmente de fontes locais, como rochas granito-gnáissicas e de greenstone belts, associadas ao Bloco do Gavião ( $>2,0$ Ga; embasamento do CSF na área de estudo), e rochas associadas a eventos vulcânicos preservados no Grupo Rio dos Remédios e formações Bomba e Tombador (aprox. 1,75 Ga, 1,5 e 1,38, respectivamente). Os resultados indicam que durante a deposição da Unidade B, a Bacia de Irecê possuía uma configuração condizente com uma bacia sag, intracratônica, possivelmente correspondendo a um golfo vinculado ao desenvolvimento da margem passiva que se implantou nas faixas Riacho do Pontal e Sergipana, a NNE.

Palavras-chave: Neoproterozoico, estratigrafia de alta resolução, datação de zircão pelo método U-Pb, plataforma carbonática, Bacia do Irecê, Cráton São Francisco 


\section{Abstract}

Santana, A.V.A. High resolution stratigraphic analysis: example in a carbonate ramp microbialite-dominated, Salitre Formation, Irecê basin, Bahia. Thesis (PhD in Geology) - Geosciences Institute, University of Brasilia, Brasília, 2016.

The Salitre Formation records a Neoproterozoic carbonate sequence overlying the São Francisco into the central region of Bahia, northeastern Brazil. This study provides an unprecedented high-resolution stratigraphic framework from partly Unit B, south syncline Irecê, into homonymous basin. Ten facies and 8 subfacies were characterized. The vertical succession show different facies associations: middle ramp with microbial influence (FA1); shallow inner to the proximal middle ramp (FA2); inner ramp microbial-dominated(FA3); subaerial ramp/sabkha (FA4), that shows a typical framework of shallow marine carbonate developed in a homoclinal carbonate ramp. Sequential analysis at high resolution distinguished elementary and small-scale sequence within intervals with a predominance of the FA3 and FA4. Elementary sequences (A-type) show typical thicknesses of the few tens of centimeters. These sequences were delineated in the field mainly from the identification of orderly factors series, such a facies or subfacies that by vertical succession demonstrated a periodicity/variability of the microbial growth. Small-scale sequences (B-type) are constituted by elementary sequences. Their demarcation occurred from sedimentological criteria and aid of the gamma ray log. Medium-scale sequences were delineated from the paleoenvironments changes. One incomplete large-scale sequence was inferred and shown tens of meters thick. This sequence suggests a more significant cyclical change of environmental conditions. Isotopic analyses of $\mathrm{C} / \mathrm{O}$ were performed on samples of lower Unit B. Slightly negative values $\delta^{13} \mathrm{C}$, close to $0.0 \%$, suggest a typical isotopic signature ocean. U/Pb SHRIMP zircon analyses were carried out in tuffacceous siltstone (tuffite) and rock with significant epiclastic contribution. The age of maximum deposition of Salitre formation is restricted by age of the youngest zircon, close to $670 \mathrm{Ma}$, obtained from the tuffite. The possible source for zircons of that age, according to the geochronological ages, are (1) Riacho do Pontal Belt, related to the distal magmatism in tectonic environment of passive margin; (2) Araçuaí-West Congo Belt, related to extensional process of large-scale, well documented in African counterpart San Francisco-Congo paloeplate (volcanism La Loiula); and (3) Brasilia Belt, related to the orogenic events. Older zircon detrital ages are mainly from local sources such as granite-gneiss rocks and greenstone belts associated with the Gavião Block (>2.0 Ga; CSF basement in the study area), and rocks associated with volcanic events preserved in the Rio Group of Remedios and Bomba and Tombador fromations (approx. $1.75 \mathrm{Ga}, 1.5$ and 1.38, respectively). The zircon age distribution of the Unit B and sedimentary record indicate that the Irecê Basin were a gulf-like basin formed in intracratonic conditions (sag basin) which it was connected with the passive margin of Riacho do Pontal and Sergipana Belts, situated to NNE.

Key-words: Neoproterozoic, high resolution stratigraphy, U-Pb zircon dating, carbonate platform, Irecê Basin, São Franscico Craton 


\section{Lista de figuras}

Figura 1. Localização da área de pesquisa e principais vias de acesso........................................ 6

Figura 2. Utilização de técnicas verticais para descrever perfis litoestratigráficos em locais com

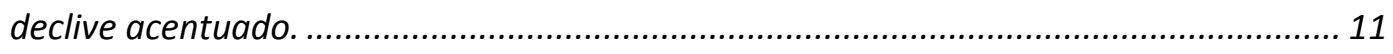

Figura 3. Modelo utilizado para descrição dos perfis litoestratigráficos................................... 13

Figura 4. Utilização de técnicas verticais para acesso à rocha e aquisição de dados para construção dos perfis gama espectral. Pedreira Rio Preto, Palmeiras, BA. 16

Figura 5. Contexto geológico simplificado do aulacógeno do Paramirim. Figura adaptada a partir de Alkmim et al (2006), Souza et al. (2003) e Guimarães et al. (2012). .28

Figura 6. Coluna estratigráfica com as sequências do Supergrupo Espinhaço na região da Chapada Diamantina. Extraída de Guadagnin et al. (2015b). 30

Figura 7. Coluna estratigráfica simplificada do Supergrupo São Francisco na região da Chapada Diamantina. Dados geocronológicos obtidos em Santana et al. (submetido)* e Figueiredo (2008)**, e significam idades máximas de deposição.

Figura 8. A trafogênese Toniana proporcionou a reativação de estruturas pré-existentes e gerou sítios deposicionais para a implantação do Supergrupo São Francisco (SSF). A Direção geral do avanço de geleiras responsáveis pela sedimentação glácio-marinha da base do SSF. B - Destaque para as importantes suturas que margeam o cráton São Francisco e principais trechos com implantação de bacias de antepaís (modificado de DOMINGUEZ, 1993). A Bacia de Irecê, seta vermelha, representa um cinturão epidérmico de antepais (REIS et al., 2013). 36

Figura 9. Unidades informais definidas para Formação Salitre na Bacia de Irecê com ciclos de raseamento ascedente identificados, de acordo com Misi (1979) e Misi e Kyle (1994). Figura adaptada a partir de Misi e Silva (1996)...

Figura 10. Unidades informais propostas para a Formação Salitre por Bomfim et al. (1985) e Pedreira et al. (1987). Figura adaptada a partir de Lagoeiro (1990). 45

Figura 11. Ciclos identificados na Formação Salitre, Bacia de Irecê, sinclinal de Irecê (SOUZA et al., 1993). A seção estudada está inserida nas subunidades Nova América Inferior e Nova América Superior, Ciclo Regressivo I, destaque.

Figura 12. Geological context. (A) Sketch map showing the location of the study area in the scenario of São Francisco Craton. (B) Simplified geological map of the Paramirim Aulacogen and their main structures. The synclines in which the Salitre Formation crops 
out are highlighted in the legend by a yellow stripe (modified from Souza et al., 2003; Guimarães et al., 2012).

Figura 13. Stratigraphic column of the São Francisco Supergroup in Irecê Basin. The Salitre Formation was subdivided into five units and organized into two shallowing-upward regional cycles (hundreds of meters thick; Misi, 1979; Misi and Kyle, 1994). The red bar indicates localization of the section studied. Others references: (1) Barbosa and Sabaté, 2004; (2) Guadagnin et al., 2015; (3) Guimarães, 1996; (4) Torquato and Misi, 1977; (5) Figueiredo (2008); (6) Santana et al., in press; (7) Misi and Veizer, 1998. Square indicates maximum depositional age for Bebedouro Formation (Figueiredo et al., 2009), and star indicates maximum depositional age for Salitre Formation (Santana, 2016). 59

Figura 14. Facies recognized in the windows study. (A) Stratiform stromatolite, finely laminated (F1a subfacies); (B) Thin section of the F1a subfacies, N//; (C) Note an increase of the porosity within finely laminated subfacies (yellow arrow) and convex microbial mats within of the laminated subfacies (F1b subfacies; white arrow); (D) Coarsely laminated (F1c subfacies) with more convex microbial mats (white arrow); (E) Stratiform stromatolite whit laminae convex (F1d subfacies). Coin for scale is $2 \mathrm{~cm}$ in diameter; (F) Stromatolite with large columns (F2b subfacies). Highlights for the loaf-shaped-type (dashed).

Figura 15. Facies recognized in the windows study (continuation). (A) Pseudobreccia with tepees (F3a subfacies); (B) Silicified pseudobreccia (F3a subfacies). Note an increase of the porosity (yellow arrow); (C) Laminite (F4 facies); (D) Cross-laminated wackestone (F6 facies); (E) Massive peloidal-ooidal packstone/wackestone (F7 facies); (F) Massive intraclastic peloidal grainstone/packstone (F8 facies). Highlights for the load (flame) structure at bed base (black arrow); (F) and (H) Intraclastic floatstone/rudstone (F9 facies). 68

Figura 16. Different elementary sequences recognized in the study area. 77

Figura 17. Visualization of a maximum flooding zone of the sequence elementary (mfz A-type). Sequence $A$ is easily recognizable in the field because its limits commonly mark discontinuities in outcrops. 78

Figura 18. Vertical arrangement of the different sequences $A$ allows observed tendency of shallowing in a sequence $B$, normally associated with the occurrence of facies indicative of less accommodation availability of a sequence $A$, where the thickness of this facies is anomalous, often with abundant structures that suggest subaerial exposure. See Table 1 for facies and subfacies descriptions and Fig. 16 to legend. 79

Figura 19. Cross-sections correlates the medium-scale sequences described. 81 
Figura 20. General ramp model and facies associations' distributions. Subdivision in accordance with Burchette and Wright, 1992.

Figura 21. Geological context. (A) São Francisco Craton in the tectonic scenario of West Gondwana (modified from Alkmim et al., 2006). (B) Detail of the central portion of the São Francisco Craton and external mobile belts. (C) Simplified geological map of the Paramirin Aulacogen with localization of the studied area (star; modified from Souza et al., 2003; Guimarães et al., 2012). 107

Figura 22. Stratigraphic column of the São Francisco Supergroup in Irecê Basin showing shallowing-upward carbonate cycles (hundreds of meters thick) identified in the Salitre Formation (Misi,1979; Misi and Kyle,1994). The red bar indicates location of the investigated section; star the analyzed sample location in this study; square indicates maximum depositional age for Bebedouro Formation (Figueiredo et al., 2009).. 110

Figura 23. Facies, $C$ and $O$ profiles in the studied section. The tuffaceous interval what has geochronological analysis it's highlighted by a yellow stripe. The outcrop photographs mentioned in the article is also displayed.

Figura 24. Characteristic examples of facies described in the Salitre Formation in the Rio Preto Quarry. (A) General view showing tabular geometry and extensive of the bodies. (B) Inset of (A), show the contacts irregular-to sharp of the different facies. (C) Horizontally laminated claystone- to siltstone, in the facies FI. (D) and (E) Levels exhibit wavy bedding (facies He). 120

Figura 25. Characteristic examples of facies described in the Salitre Formation in the Rio Preto Quarry (continuation). (A) and (B) Horizontally laminated in the tuffaceous siltstone- to mudstone, facies Fl(v). (C) Main petrographic characteristics of the facies Fl(v) are abundant crystals clast immersed in a very-fine-grained matrix. (D) Crinkly laminite facies highlight to occurrence the karst topography (arrow). (E) Fine-to very fine-grained carbonate levels arranged in irregular to wrinkled lamination with $\mathrm{mm}$-scale, facies $\mathrm{Lm}$.

Figura 26. Geochemical plots for the tuffite layer. A - A volcanic rocks discrimination diagram (Winchester and Floyd, 1977) with tuffite sample plotted as signature of the magma with a trachyandesitic composition. Taylor and McLennan (1985) and Formoso et al. (1999) show the data used for the comparison. B - Data plotted in the specific multielement diagram normalized to chondrite (Thompson, 1982). C - Spider diagram of the REE normalized to the chondrite (Boynton, 1984) which indicates the tuffite layer and reference values from different rocks of the Proterozoic obtained in Condie (1993) and

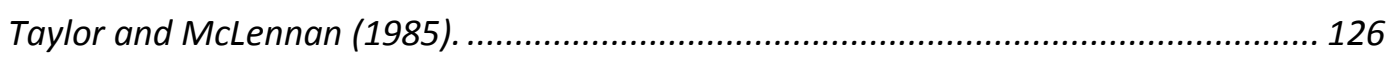

Figura 27. Cathodoluminescence images of the zircon grains from the tuffite sample..... 128 
Figura 28. Histogram with U-Pb ages from detrital zircons of Salitre Fm., Irecê Basin, Bahia 131

Figura 29. Normalized age probability histogram of the detrital zircon U-Pb ages from

Figura 30. Schematic chart illustrating the evolution of the Irecê Basin from 720 Ma to > 580 Ma showing the A. rifting (glacial deposits of the Bebedouro Fm.) associated with fragmentation of Paleoplate São Franscico-Congo; B. restricted sea with deposition of Cap dolomite of the basal Salitre Fm. (Unit C) and deposition of deep to middle ramp in open sea connected to the passive margin (Unit B). This sequence is covered by the foreland deposits (Unit A, A1, see figure 22), which it is not represented in this sketch (see text for explanation).

142 


\section{Lista de quadros e tabelas}

Quadro 1. Síntese com as unidades litoestratigráficas propostas para Formação Salitre49

Table 1. Summary off the main characteristic features of the facies types identified in the Salitre Formation in this study area (Article 1)

Table 2. Summary off the main characteristic features of the facies types identified in the Salitre Formation in this study area (Article 2)

Table 3. $\mathrm{C}$ and $\mathrm{O}$ isotopes values, $\mathrm{Mn}$ and Sr contents (ppm) and geochemical ratios obtained on analyzed carbonates. Error $\delta^{13} \mathrm{C}=0,05 \% ; \delta^{18} \mathrm{O}=0,10 \%$ 123

Table 4. Summary of SHRIMP U-Pb zircon data for samples PRP-16 (Rio Preto Quarry) and PEC-01 (Pecuária Cave)..... 


\section{SUMÁRIO}

1 INTRODUÇÃO ............................................................................ 1

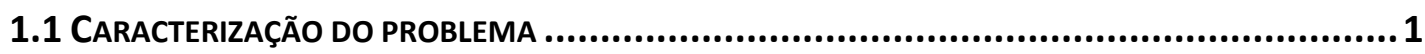

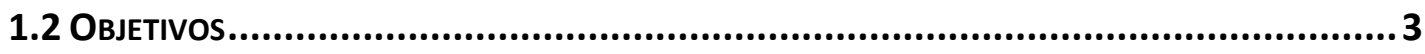

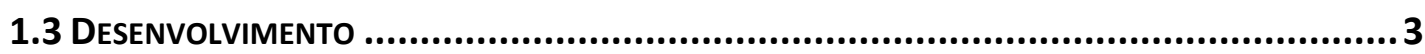

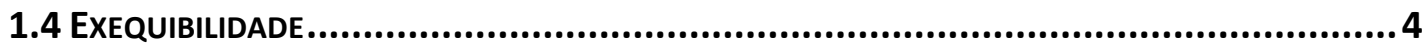

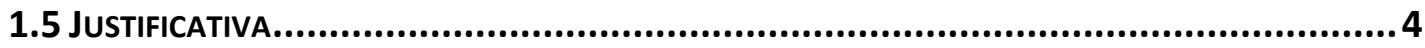

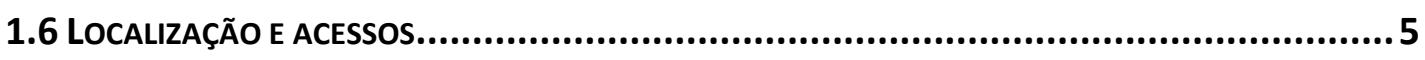

1.7 MATERIAIS E MÉTOdOS ........................................................................ 7

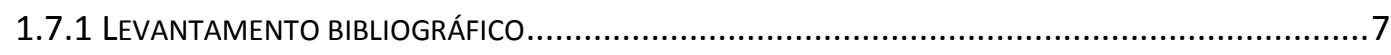

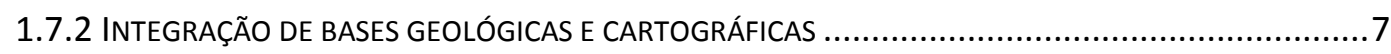

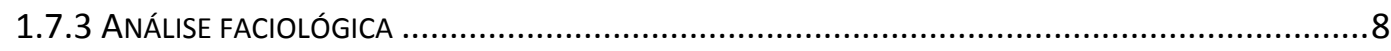

1.7.4 LEVANTAMENTO DE PERFIS GAMA ESPECTRAL ...........................................................13

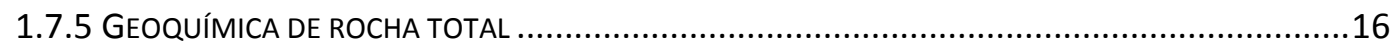

1.7.6 ESTRATIGRAFIA DE SEQUÊNCIAS DE ALTA RESOLUÇÃO ...................................................17

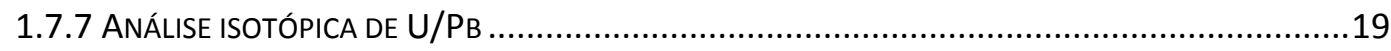

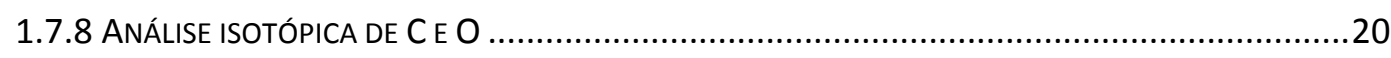

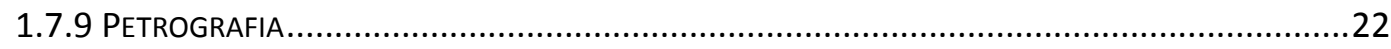

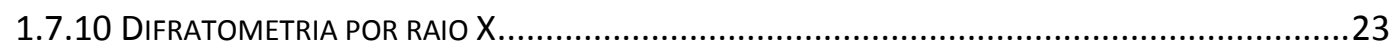

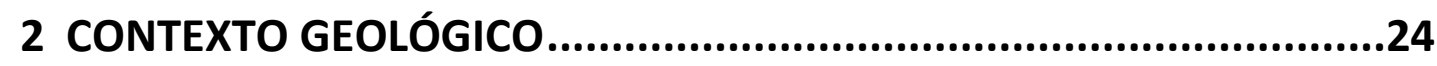

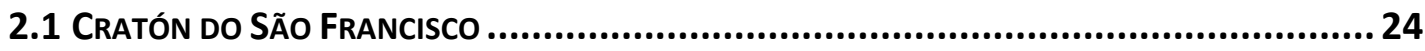

2.2 Coberturas do Cratón do São Francisco .............................................. 26

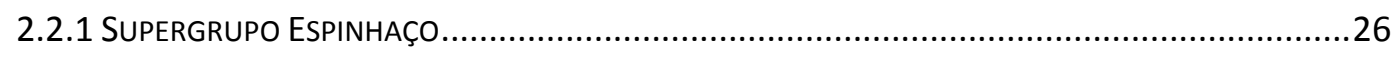


3 FORMAÇÃO SALITRE - EVOLUÇÃO DO

CONHECIMENTO

\section{CRITERIA TO HIGH-RESOLUTION STRATIGRAPHY OF A}

MICROBIAL-DOMINATED CARBONATE RAMP SYSTEM, NEOPROTEROZOIC SALITRE FORMATION, IRECÊ BASIN, NORTHEASTERN BRAZIL. .50

4.1 INTRODUCTION 53

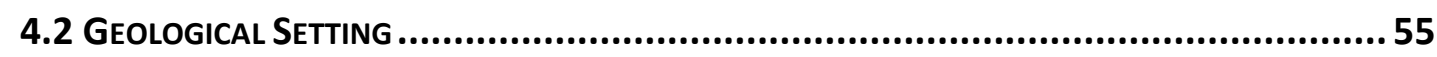

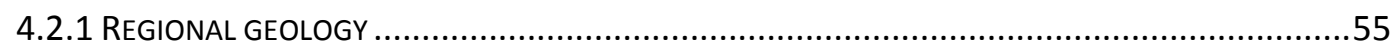

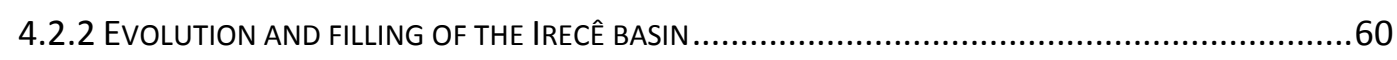

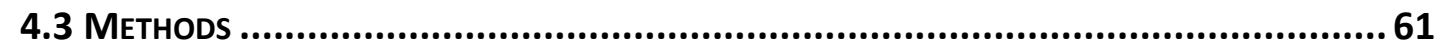

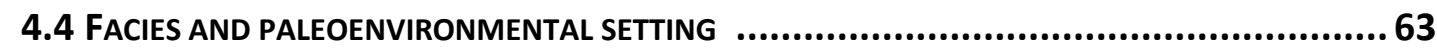

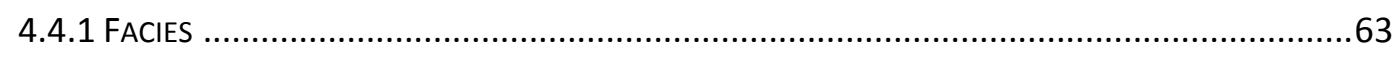

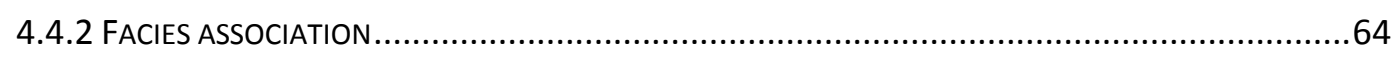

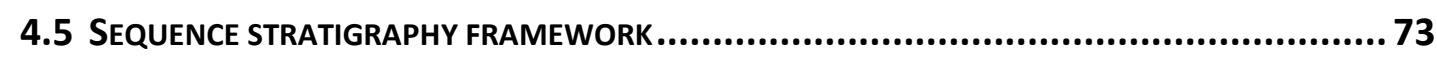

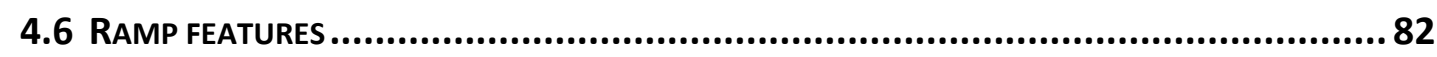

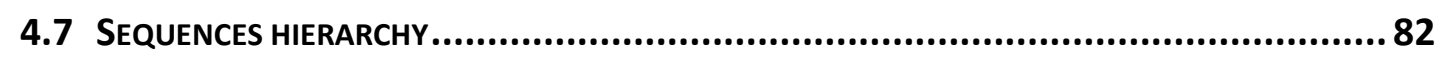

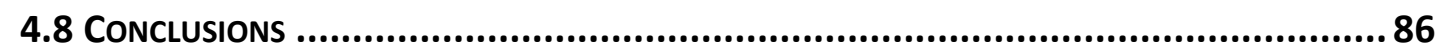




\section{NEW INSIGHTS OF GULF CARBONATE}

\section{SEDIMENTATION OF THE IRECÊ BASIN, SÃO}

FRANCISCO CRATON, BASED ON STRATIGRAPHIC

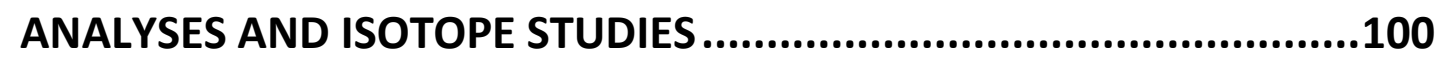

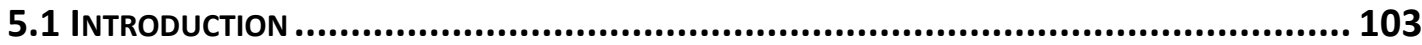

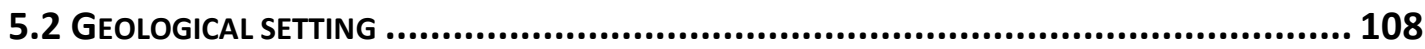

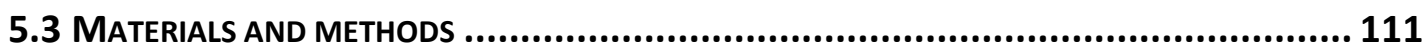

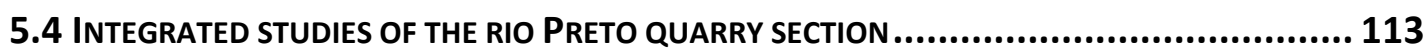

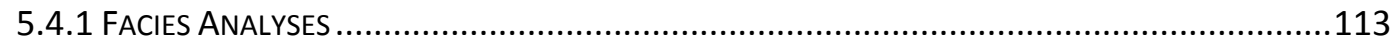

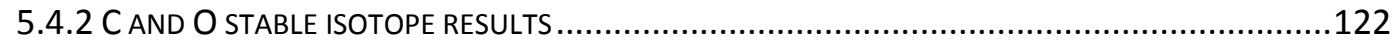

5.4 .3 GEOCHEMISTRY OF THE TUFFITE LAYER ........................................................ 124

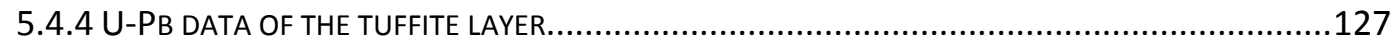

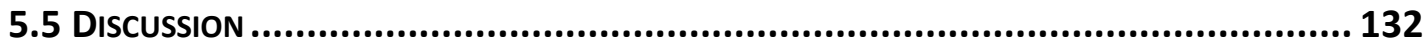

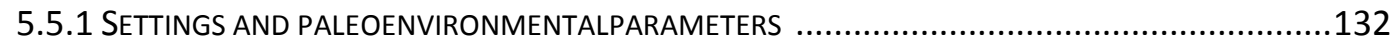

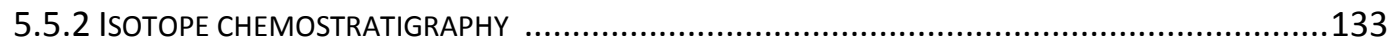

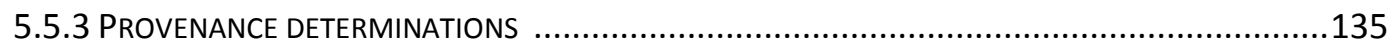

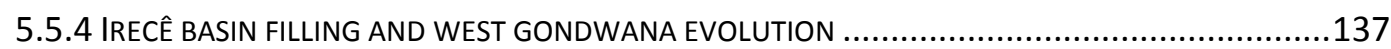

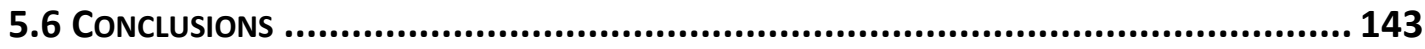

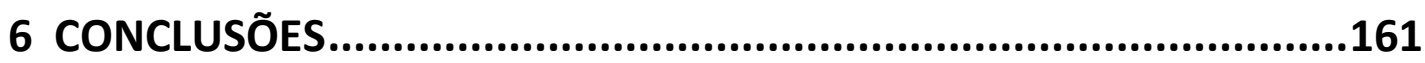

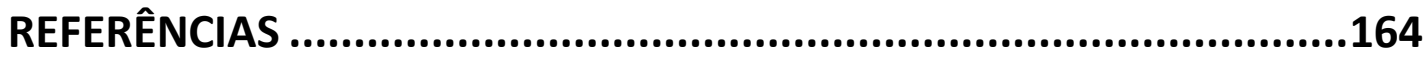

\section{APÊNDICES}




\section{TEXTO EXPLICATIVO- ESTRUTURAÇÃO DA TESE}

A presente tese está estruturada em capítulos. O primeiro tem caráter introdutório e fornece um panorama geral da pesquisa. Contêm itens sobre a caracterização do problema abordado, objetivos, desenvolvimeto e exequibilidade da pesquisa, e também discorre sobre as justificativas para execução do trabalho e municia o leitor de informações sobre a localização da área pesquisada. Além destes aspectos, o primeiro capítulo elenca, de maneira sumária, os métodos empregados para aquisição e tratamento dos dados. O segundo capítulo traz uma breve revisão sobre o contexto geológico regional. A evolução sobre o conhecimento litoestratigráfico da Formação Salitre é abordada no terceiro capítulo. Os resultados da pesquisa podem ser verificados nos capítulos 4 e 5 . Estes capítulos compreendem dois artigos científicos submetidos a periódicos internacionais. O Capítulo 4 "Criteria to high-resolution stratigraphy of a microbial-dominated carbonate ramp system, Neoproterozoic Salitre Formation, Irecê Basin, northeastern Brazil" versa sobre critérios estabelecidos para delimitação de sequências em alta resolução em um contexto de rampa carbonática dominada por microbialitos. O Capítulo 5 - "New insights of gulf carbonate sedimentation of the Irecê Basin, São Francisco Craton, based on stratigraphic analyses and isotope studies" - reporta dados inéditos de datação U-Pb SHRIMP. À exceção daquelas contidas nos manuscritos submetidos (cap. 4 e 5), todas as citações bibliográficas e as suas referências estão conforme a Norma Técnica NBR 10520 da Associação Brasileira de Normas Técnicas (ABNT). O presente documento constitui um dos requisitos para obtenção do título de doutora em Geologia e está estruturado em conformidade com o regulamento do Programa de Pós-graduação em Geologia da Universidade de Brasília. 


\section{INTRODUÇÃO}

\subsection{CARACTERIZAÇÃo dO PROBLEMA}

A Bacia do Irecê ocupa expressiva área na região centro-norte do estado da Bahia, nordeste do Brasil, perfazendo cerca de $31.000 \mathrm{~km}^{2}$. Ali afloram rochas siliciclásticas depositadas em um contexto de transição continente - plataforma, associadas aos depósitos glácio-marinhos da Formação Bebedouro (OLIVEIRA e LEONARDOS, 1943; GUIMARÃES, 1996), e rochas essencialmente carbonáticas, depositadas em ambiente marinho e mapeadas como Formação Salitre (BRANNER, 1911). Estas formações compõem o Grupo Una que, na região citada, representa o Supergrupo São Francisco (Neoproterozoico). A Formação Salitre, objeto da tese, tem arcabouço litoestratigráfico construído a partir da identificação de unidades informais que, dispostas verticalmente, compõem "ciclos" da ordem de centenas de metros de espessura, correlacionáveis em escala de bacia (SOUTO e MISI, 1975; MISI, 1979; BOMFIM et al., 1985; SOUZA et al., 1993).

Em um primeiro momento, e até a presente data, as investidas para uma melhor caracterização e entendimento estratigráfico dessa formação ocorreram principalmente devido aos bens minerais nela contidos, fato que implicou em pesquisas com abordagem regional e focadas em correlacionar metalotectos interbacias (por exemplo, MISI et al., 2007) e em estudos realizados a fim de entender o metalotecto (por exemplo, KYLE e MISI, 1997). Estes estudos se restringiram às áreas próximas de ocorrências e/ou depósitos de substâncias como $\mathrm{Zn}, \mathrm{Pb}, \mathrm{Ba}$ e $\mathrm{P}$, áreas que comumente têm uma intrigante componente estrutural, fator por vezes impeditivo para realização de estudos estratigráficos mais 
acurados, pois acarreta em imprecisão nas espessuras das unidades e falta de continuidade de afloramentos.

A partir do cenário acima delineado, as provocações iniciais para executar a pesquisa foram duas, (1) a não existência de trabalhos em escala de detalhe, executados em áreas pouco deformadas da bacia e construídos sob a ótica de estratigrafia de sequência em alta resolução (ESAR), método utilizado na tese, e (2) carência de estudos com tais características em rochas carbonáticas no Brasil. Durante o desenvolvimento dos estudos, mais uma lacuna ficou evidente: a ausência de um arcabouço geocronológico robusto para a Formação Salitre. E esse foi um objetivo acrescentado, já com o avançar da pesquisa.

Optar por construir um arcabouço estratigráfico utilizando o método ESAR significa admitir que o mesmo possui um significado genético e temporal, absoluto ou relativo. Este arcabouço terá diferentes intervalos, referidos como "sequência" e delimitados por superfícies-chave. O conceito de sequência adotado na pesquisa é aquele verificado em Catuneanu et al. (2009): "uma sucessão de camadas depositadas durante um ciclo completo aonde há mudança na acomodação ou no suprimento sedimentar". Esta conceituação é genérica e abrange qualquer escala espacial ou temporal (CATUNEANU et al., 2011).

Quanto aos dados geocronológicos, uma breve revisão na bibliografia revela que por muito tempo toda menção de idade deposicional para a Formação Salitre esteve vinculada ao que se conhecia para o Grupo Bambuí, em Minas Gerais e no oeste da Bahia, pois, por muito tempo, não houve dissociação entre essa e aquele. Com efeito, as primeiras referências 
diretamente associadas à Formação Salitre são do início dos anos 1980 - Srivastava (1982, 1986) - e têm a bioestratigrafia como suporte. Baseados em estromatólitos que ocorrem no registro, estes estudos limitaram a deposição da formação ao Neoproterozoico. Datações mais restritivas foram verificadas somente após aplicações de métodos radiométricos com a execução dos trabalhos de Toulkeridis et al., 1999 (700 Ma; Rb/Sr em clorita autigênica), Santos et al., 2011 (1.705 Ma; U/Pb em zircão detrítico).

\subsection{OBjetivos}

Os principais objetivos da pesquisa foram (1) fornecer um arcabouço de estratigrafia em alta resolução para as unidades informais da Formação Salitre, mais especificamente aquelas que afloram na região sul da sinclinal de Irecê, e (2) estabelecer as idades máximas de deposição e possíveis áreas-fonte para a Unidade $B$.

\subsection{DeSENVOLVIMENTO}

O desenvolvimento da tese ocorreu ao longo de 8 semestres letivos e foi dividido em diferentes etapas. A primeira etapa abrangeu uma pesquisa nas principais referências bibliográficas sobre geologia regional e evolução tectônica de bacias, e sobre sedimentologia e estratigrafia em rochas carbonáticas do Pré-cambriano. Nesta etapa também houve a aquisição e integração de diferentes bases geológicas, cartográficas e de imagens geradas por sensores remotos. Após, foi estabelecida a base metodológica para aquisição dos dados em campo. A etapa seguinte constou de estudos petrográficos. Na etapa final houve a integração e interpretação dos resultados, elaboração de manuscritos e redação da tese. 


\subsection{EXEQUIBILIDADE}

A exequibilidade da pesquisa foi possível devido ao apoio técnico e financeiro dos projetos "Análise estratigráfica de alta resolução em sistemas carbonáticos microbiais do Neoproterozoico - Exemplo da Formação Salitre, BA" e "Estratigrafia de sequências de alta resolução em rochas calcárias do Neoproterozoico - desenvolvimento de método aplicável ao zoneamento de campos do Pré-sal", ambos executados pela Universidade Federal do Rio Grande do Sul, em parceria com a empresa Petróleo Brasileiro S.A (PETROBRAS), com verba da Lei de Participação Especial 9.478/1997 da Agência Nacional do Petróleo, Gás Natural e Biocombustíveis (ANP). A autora da tese atua como coordenadora técnica destes projetos. 0 Conselho Nacional de Desenvolvimento Científico e Tecnológico (CNPq) disponibilizou a verba empregada para as análises geoquímicas em rocha-total, realizadas no Acme Analytical Laboratories Ltd, e para as análises isotópicas de U/Pb com o equipamento SHRIMP II, realizadas na Western Australia University e na Universidade de São Paulo. O CNPq também forneceu auxílio na forma de bolsa de pesquisa e taxa de bancada (Processo $160391 / 2011-3)$

\subsection{JUSTIFICATIVA}

A tese contribui para o aprimoramento de métodos de ESAR em sistema carbonático microbial, fato que deve impactar em melhor entendimento sobre a deposição da unidade estudada, Formação Salitre, e em melhor compreensão sobre os fatores que regem o desenvolvimento de formações carbonáticas análogas ou similares, de diferentes idades. Os dados inéditos de isótopos irão auxiliar para melhor compreensão da evolução geotectônica da bacia. 


\subsection{LOCALIZAÇÃO E ACESSOS}

A área de estudo está situada na região centro-oriental da Chapada Diamantina, estado da Bahia. Estende-se por aproximadamente $120 \mathrm{~km}^{2}$ englobando parte dos municípios de Iraquara, Palmeiras e Seabra, segundo as coordenadas geográficas 465'ㄷ' W - 4651'7,2" W / 16059'20,4" S - 172'27,6" S (Figura 1). Está inserida parcialmente nas folhas topográficas Seabra (SD. 24-V-A-I), Utinga (SD. 24-V-A-II) e Palmeiras (SD. 24-V-A-IV). O principal acesso rodoviário à região, a partir de Salvador, é feito ao longo das rodovias federais BR-324, BR-116, BR-242 e BR-122, em um percurso total de aproximadamente 450 km. Há também acesso aéreo com voos regulares da capital baiana para Lençóis, cidade que dista cerca de $60 \mathrm{~km}$ da área de estudo. 0 ingresso às seções verticais é feito por estradas vicinais, trilhas e caminhos cujos acessos são realizados principalmente a partir da BR-122. 

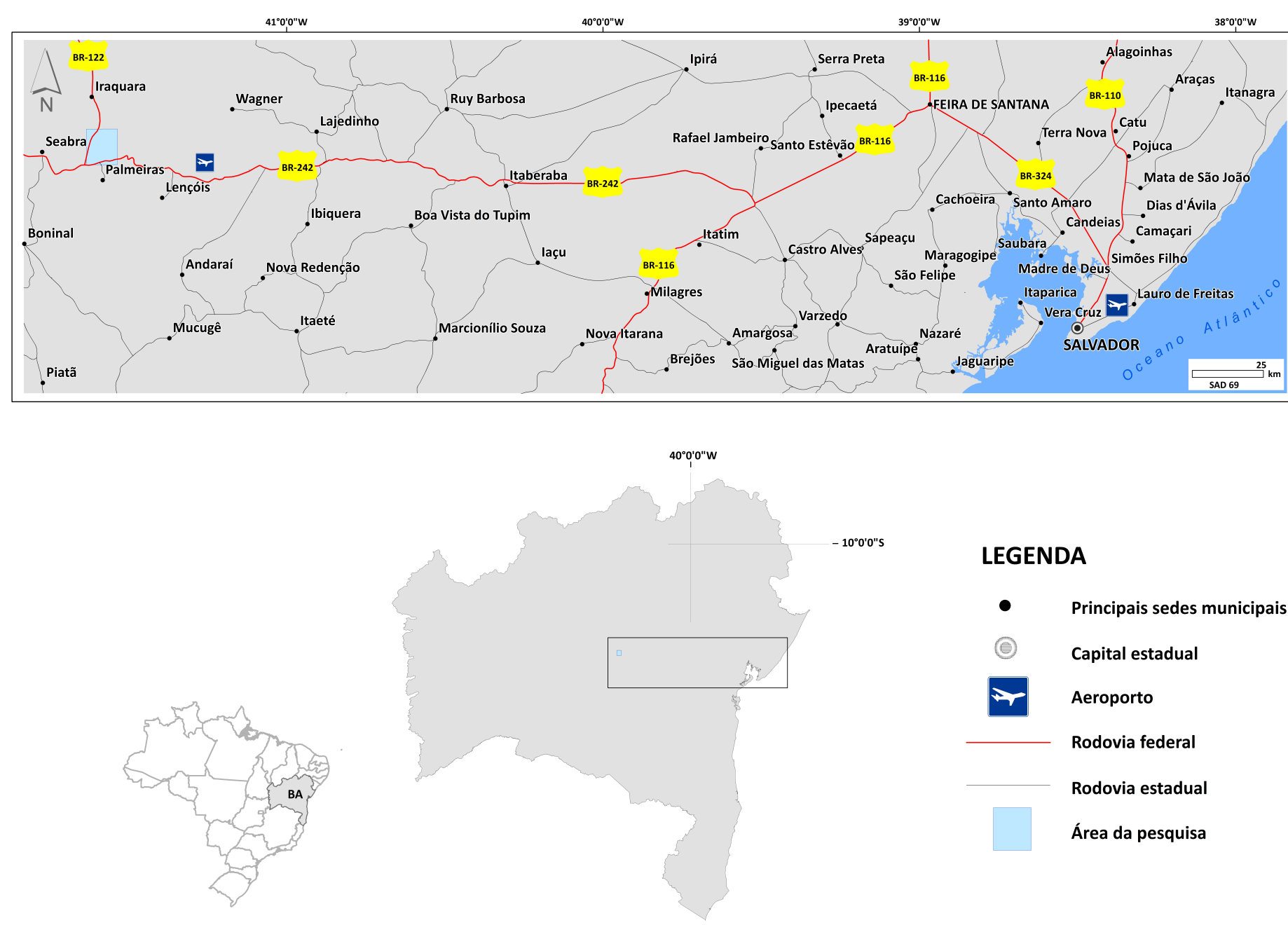

LEGENDA

- $\quad$ Principais sedes municipais

(P) Capital estadual

Aeroporto

Rodovia federal

Rodovia estadual

Área da pesquisa

Figura 1. Localização da área de pesquisa e principais vias de acesso. 


\subsection{MATERIAIS E MÉTODOS}

Para aquisição, integração e interpretação dos dados foram utilizados diversas ferramentas e métodos, descritos a seguir, de forma sucinta.

\subsubsection{LeVANTAMENTO BIBLIOGRÁFICO}

Análise bibliográfica foi realizada em periódicos e livros, nacionais e internacionais, com vistas à seleção dos trabalhos considerados fundamentais sobre o tema da pesquisa e os aspectos da geologia regional. O material selecionado foi devidamente compilado, catalogado e retido em formato Portable Document Format (.pdf) com auxílio do software Mendeley ${ }^{\circledR}$ - Mendeley (2011).

\subsubsection{INTEGRAÇÃO DE BASES GEOLÓGICAS E CARTOGRÁFICAS, SENSORES REMOTOS E FOTOGRAFIAS AÉREAS}

Para definir potenciais afloramentos foram utilizadas diversas bases de dados: (i) Imagens de interferometria de radar SRTM (Shuttle Radar Topography Mission - JPL, 2011), de satélite LANDSAT 5 (Land Remote Sensing Satellite - INPE, 2010) e imagem aérea ortorretificada na escala 1:10.000; (ii) mapas planialtimétricos e geológico, adquiridos em sites de órgãos governamentais e (iii) fotografias aéreas. A seleção dos afloramentos foi baseada no estudo do arcabouço estratigráfico regional e na identificação de áreas mais propícias às boas exposições rochosas, normalmente relacionadas ao relevo cárstico - dolinas e vales cegos -, e às escarpas próximas aos rios. Programas específicos, como o $\operatorname{ArcGIS} 10^{\circledR}$ (ESRI, 2010) e Global Mapper $12^{\circledR}$, serviram para integrar as diferentes bases. 


\subsubsection{ANÁLISE FACIOLÓGICA}

A análise faciológica ocorreu a partir do levantamento em campo de perfis colunares em escala de detalhe (1:20), posteriormente correlacionados através do software PETREL ${ }^{\circledR}$. As fácies foram descritas, macro e mesoscopicamente, conforme método proposto em Walker e James (1992). A definição para "fácies" adotada na pesquisa é aquela verificada em Reading (1996): fácies é um corpo de rocha (camada ou múltiplas camadas) com características específicas representadas por parâmetros diagnósticos como cor, tamanho do grão, composição, estruturas internas, geometria deposicional, espessura, fósseis e paleocorrentes. A correta descrição desses parâmetros, o estado de conservação das rochas analisadas e o volume de dados disponíveis conferem maior ou menor confiabilidade à análise sedimentológica-estratigráfica.

No presente estudo, além dos parâmetros acima mencionados, foram estabelecidos critérios adicionais para melhor caracterizar as fácies identificadas. Um desses critérios, de fundamental importância, foi identificar na ocorrência de formas bioconstruídas Microbialito sensu Burne e Moore (1987) - a morfologia e a proporção dos seus diferentes tipos/fábrica. Esta divisão tornou-se necessária para compreensão dos ciclos básicos na análise sequencial de alta resolução e será detalhada no Capítulo 4.

O código adotado durante a descrição das fácies carbonáticas, retrabalhadas ou bioconstruídas, está disposto em Terra et al. (2010), trabalho que reúne, adapta e modifica alguns termos das principais propostas para classificação de carbonatos vigentes (FOLK, 
1962; DUNHAM, 1962; EMBRY e KLOVAN, 1971; RIDING 2000), bem como introduz novas denominações. No entanto, à semelhança de classificações faciológicas para rochas siliciclásticas, como o código proposto por Miall (1977) para sistemas fluviais, no presente estudo será destacada a textura dominante da fácies em letras maiúsculas acrescida de letra minúscula para designar a principal estrutura presente. Feições modificadoras, como dolomitização ou silicificação, também são inclusas no código, antes das maiúsculas, com a letra inicial em minúsculo. Assim, por exemplo, o código para um dolarenito com textura grainstone e estratificação cruzada planar (planar cross-bedding) será "dGSTp".

Para as raras ocorrências de fácies siliclásticas/impuras, adotou-se o código de Miall (1977) e, quando há presença de contribuição vulcânica, esse código foi acrescido com a letra "v". Fácies tectônica, formadas a partir de fraturamento hidraúlico devido à presença de veios e vênulas de calcita, são mencionadas nos perfis, mas não foram inseridas no desenho dos logs. Além desses códigos, são propostos códigos operacionais para subdivisão das formas microbiais (subfacies), descritos no Capítulo 4.

As seções colunares foram adquiridas com o auxílio de trena e régua estratigráfica e, em perfis com elevados gradientes de declividade, foram utilizadas técnicas verticais - rapel e escalada - para acesso à rocha (Figura 2). A descrição das fácies foi acompanhada pelo imageamento fotográfico convencional utilizando máquina digital NIKON 5100 e lente SIGMA NIKON 50/500 mm. Durante o levantamento dos perfis realizou-se amostragem sistemática para estudos petrográficos e procedimentos analíticos. 
Para a construção do perfil colunar em campo, foi utilizada uma ficha de descrição adaptada de um modelo empregado para descrição de carbonatos disponibilizado pela PETROBRAS (Figura 3). A fim de representar conjuntamente as fácies essencialmente carbonáticas e as siliciclásticas, o eixo horizontal consta de variação da granulação, de acordo com Wentworth (1922), que abrange o tamanho dos componentes das diferentes fácies. Há também indicação do local de preenchimento para algumas fácies carbonáticas, de acordo com textura predominante. Os microbialitos, por exemplo, foram plotados na granulação silte. No eixo vertical é representada a espessura de cada estrato.

No modelo adotado, o cabeçalho apresenta espaços para o preenchimento de informações básicas de cada afloramento. O restante da folha é utilizado para o preenchimento da coluna estratigráfica, conforme visto anteriormente, além de informações associadas com o código e descrição das fácies, desenhos, medidas estruturais, como o acamamento sedimentar $\left(\mathrm{S}_{0}\right)$, estruturas sedimentares, geometria, amostras e fotos. Neste modelo também há campos específicos para a indicação da ciclicidade de alta frequência visualizada em campo. Esta ciclicidade é assinalada a partir de quebras significativas no registro, geralmente abruptas e com desenvolvimento de estruturas de dissolução ou exposição, e/ou a partir da repetição cíclica de fácies. Também foram incluídas as interpretações das associações de fácies e superfícies limítrofes, visando à reconstrução da arquitetura deposicional das unidades estratigráficas estudadas. 


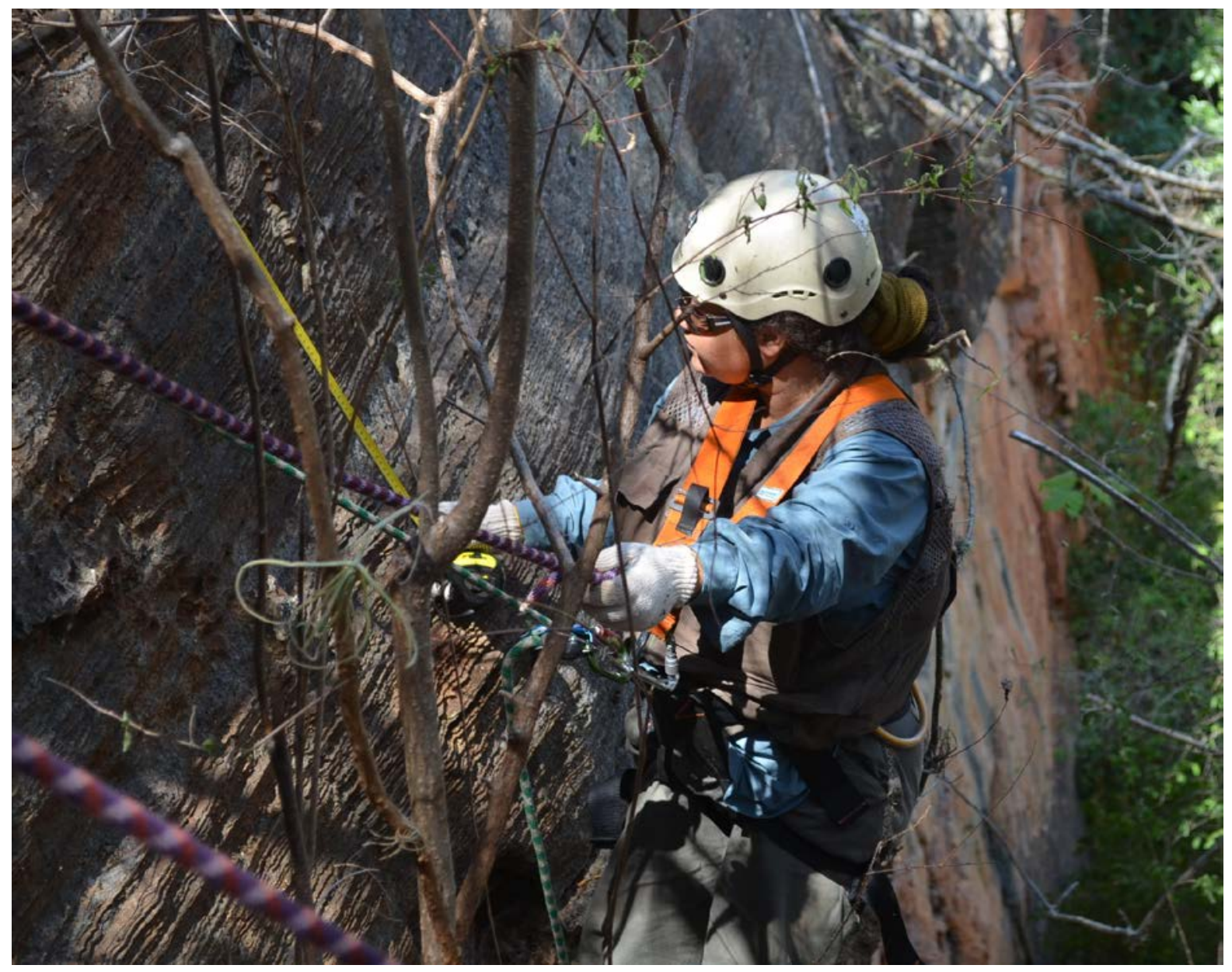

Figura 2. Utilização de técnicas verticais para descrever perfis litoestratigráficos em locais com declive acentuado.

Os perfis foram digitalizados, utilizando o software de análise sequencial, ANASETE ${ }^{\circ}$, desenvolvido pela PETROBRAS, e depois foram inseridos no software PETREL ${ }^{\circ}$. Alguns foram redesenhandos com o software Adobe Illustrator ${ }^{\bullet}$, que permite uma representação gráfica mais fiel dos dados verificados em campo.

Definidas as fácies, e entendidos os processos responsáveis por sua formação, foi estabelecido um agrupamento de fácies cogenéticas, ou seja, uma associação de fácies com correlação lateral e/ou vertical. A proposição de uma associação faciológica implica na 
admissão que as condições ambientais durante a deposição das fácies que a compõem guardam semelhanças entre si, sejam elas físicas, químicas, ou ambas. O conceito de associação de fácies foi originalmente discutido no trabalho de Collinson (1969 in WALKER e JAMES 1992) e, desde então, tem sido largamente empregado em estudos sedimentológicos/estratigráficos.

A inferência do modelo deposicional conceitual, por sua vez, resultou da síntese de todas as relações e análises genéticas e tridimensionais das fácies. Estas relações foram baseadas em dados do Recente e registros sedimentares antigos. O modelo fornece um arcabouço geral, na forma de bloco-diagrama, e permite a observação da relação entre os diferentes paleoambientes/subsistemas, elecandos com a denominação de associação de fácies. Fotomosaicos foram construídos e interpretados para destacarem, especialmente, a geometria dos principais ciclos e associações de fácies descritas, bem como para ressaltar superfícies-chave. A construção seguiu o método proposto por Wizevich (1991): fotografias obtidas com distância e ângulos constantes em relação ao afloramento e com um recobrimento de aproximadamente $40 \%$ entre cada fotografia, e visou preservar a escala e minimizar as distorções angulares entre as fotografias. Para a confecção e interpretação dos fotomosaicos foram utilizados os softwares Adobe Photoshop ${ }^{\circledR}$ e Adobe Illustrator ${ }^{\circledR}$, respectivamente.

\subsubsection{LEVANTAMENTOS DE PERFIS GAMA ESPECTRAL}

Perfis gama espectral são amplamente utilizados na indústria de hidrocarbonetos para interpretar litologias e efetuar correlações em subsuperfície e, mesmo existindo diferenças entre levantamentos em subsuperfície e em afloramentos, a possível discrepância não 
invalida a interpretação rocha-perfil possibilitada pelo método (DAVIES e ELLIOTT, 1996). O príncipio de funcionamento deste método consiste em, a partir da detecção da radiação natural emitida pelos principais elementos radioativos - isótopo de potássio $\left(\mathrm{K}^{40}\right)$ e elementos das séries do urânio (U) e do tório (Th) -, gerar perfis em que sejam visualizadas as assinaturas/tendências dos radioelementos citados, além do parâmetro contagem total. Estes perfis, aliados a dados litológicos, permitem inferir sobre argilosidade, teores de matéria orgânica e qualificar a presença de degradação oxidativa (ULBRICH et al., 2009).

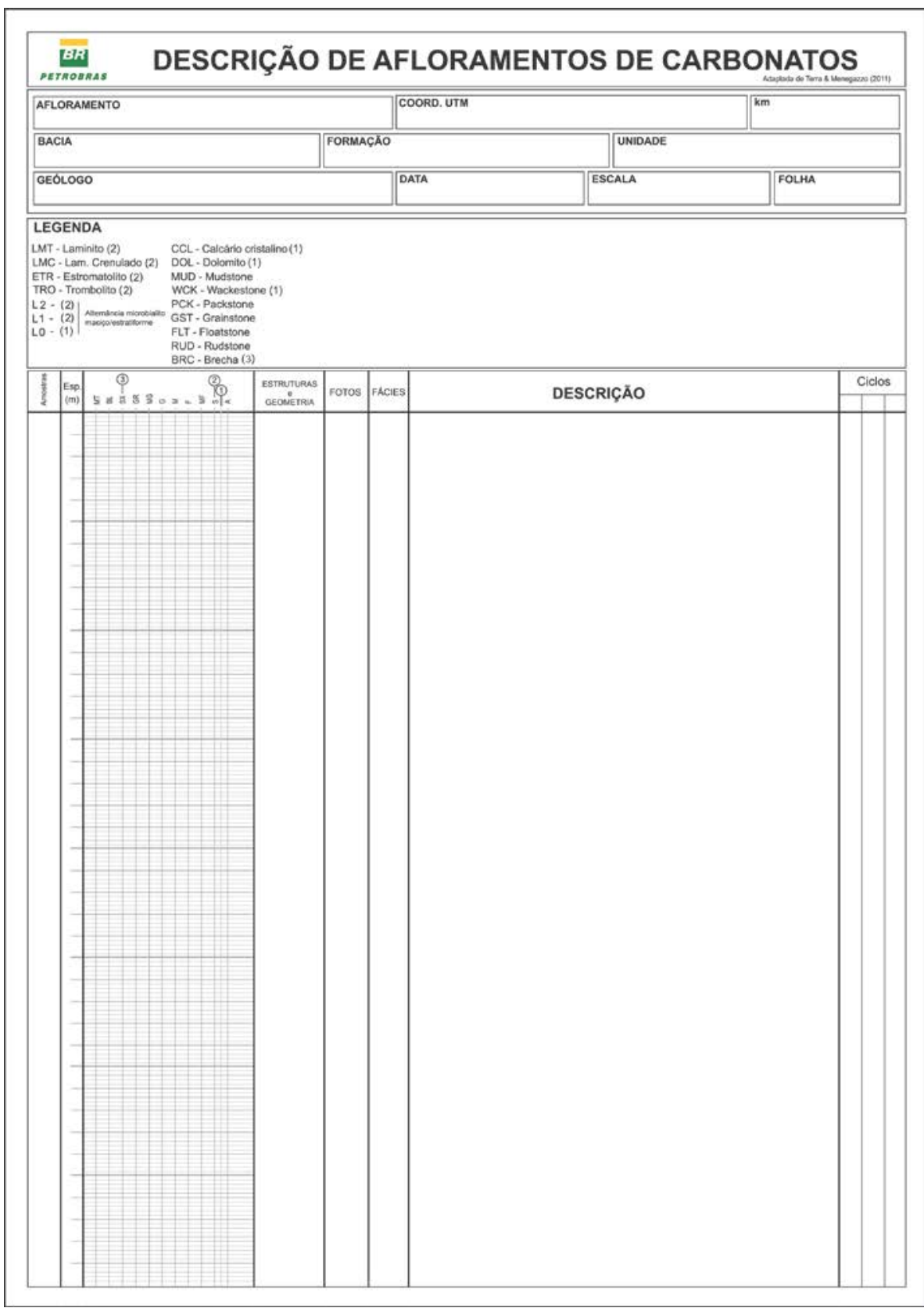

Figura 3. Modelo utilizado para descrição dos perfis litoestratigráficos. 
Na natureza a concentração de cada um desses elementos reflete uma condição específica: K está presente em K-felsdpatos, micas e argilominerais do grupo da ilita, Th se concentra em minerais pesados, como zircão e monazita, ou em associação com certos argilominerais e com fosfatos autigênicos (HURST, 1990; HERRON e MATTERSON, 1993) e U, por sua vez, faz parte da constituição de minerais pesados comuns em sedimentos anóxicos (LOVLEY et al., 1991). Em rochas calcárias/dolomíticas, K e Th normalmente estão associados às concentrações de minerais insolúveis residuais, ou aos minerais autigênicos, e U representa, principalmente, o teor de matéria orgânica e a concentração de alguns minerais autigênicos. Os radioelementos Th e K são utilizados na diferenciação da fábrica carbonática, se dominada por grãos ou por lama, e são utilizados em correlações laterais de eventos deposicionais. $U$ é fortemente afetado por processos diagéneticos e deve ser analisado com parcimônia, já que pode não representar a fábrica/fácies deposicional. Normalmente são realizadas análises a fim de verificar a concentração de $U$ em relação aos demais elementos para averiguar se há uma correlação positiva e, assim, utilizar o elemento com maior confiabilidade nas correlaçães estratigráficas (LUCIA, 2007).

Os levantamentos de perfis gama espectral foram realizados de maneira sistemática, em intervalos com espaçamentos regulares de $20 \mathrm{~cm}$, ou próximos, seguindo uma linha ortogonal ao acamamento primário da rocha, a fim de obter a espessura real do intervalo, e com o aparelho disposto de forma paralela a este acamamento, configuração que capta melhor a radiação emitida pela rocha. O equipamento utilizado foi do tipo portátil, modelo RS-230BGO Super-Spec ${ }^{\circledR}$ (Radiation Solutions Inc.), emprestado pela Universidade PETROBRAS, por intermédio da Rede de Caracterização e Modelagem de Reservatórios (CARMOD), ou pela Universidade Federal de Santa Catarina (UFSC), por intermédio do 
professor Breno Waichel (Projeto Rifte II - PETROBRAS/UFSC e UFRGS). Este equipamento possui um detector de óxido de bismuto germanato de $103 \mathrm{~cm}^{3}$ que confere um ganho significativo na sensibilidade de aquisição, quando comparado aos detectores mais comuns.

As aquisições foram realizadas, sempre que possível, em locais com pouca ação de intemperismo e cada medida ocorreu com um tempo de 60 segundos. Além do dado de gama total $\left(n g V h^{-1}\right)$, foram obtidas medidas de $K(\%), U(p p m)$ e Th (ppm). A aquisição das medidas foi feita quase sempre com suporte de técnicas verticais para acesso à rocha (Figura 4). As leituras foram anotadas em planilhas confeccionadas para este fim e, ainda em campo, correlacionadas com o perfil litológico correspondente e visualizados com o uso do software RSAnalyst $^{\circledR}$. No escritório, os dados foram tabulados em planilhas do aplicativo EXCELL e armazenados nos softwares ANASETE $^{\circledR}$ e PETREL ${ }^{\circledR}$.

A resposta do parâmetro contagem total de radiação gama foi utilizada qualitativamente e, somada ao perfil litológico, forneceu informações valiosas para a interpretação da ciclicidade em alta frequência e posicionamento de superfícies estratigráficas. Perfis gama espectral têm sido empregados com sucesso em estudos de análagos de reservatório, inclusive com aplicação em potenciais análogos para seção "Pré-Sal", como o intervalo paleocênico da Formação Yacoraite, Sequência Balbuena IV, Salta, AR. Trabalhos recentes aliam dados de perfis gama espectral e litológicos em calcários microbiais lacustres e utilizam as assinaturas dos radioelementos para correlacionar e calibrar importantes superfícies estratigráficas por distâncias superiores a 40 km (RAJA GABAGLIA et al., 2011; BENTO FREIRE et al., 2011; BENTO FREIRE, 2012). 


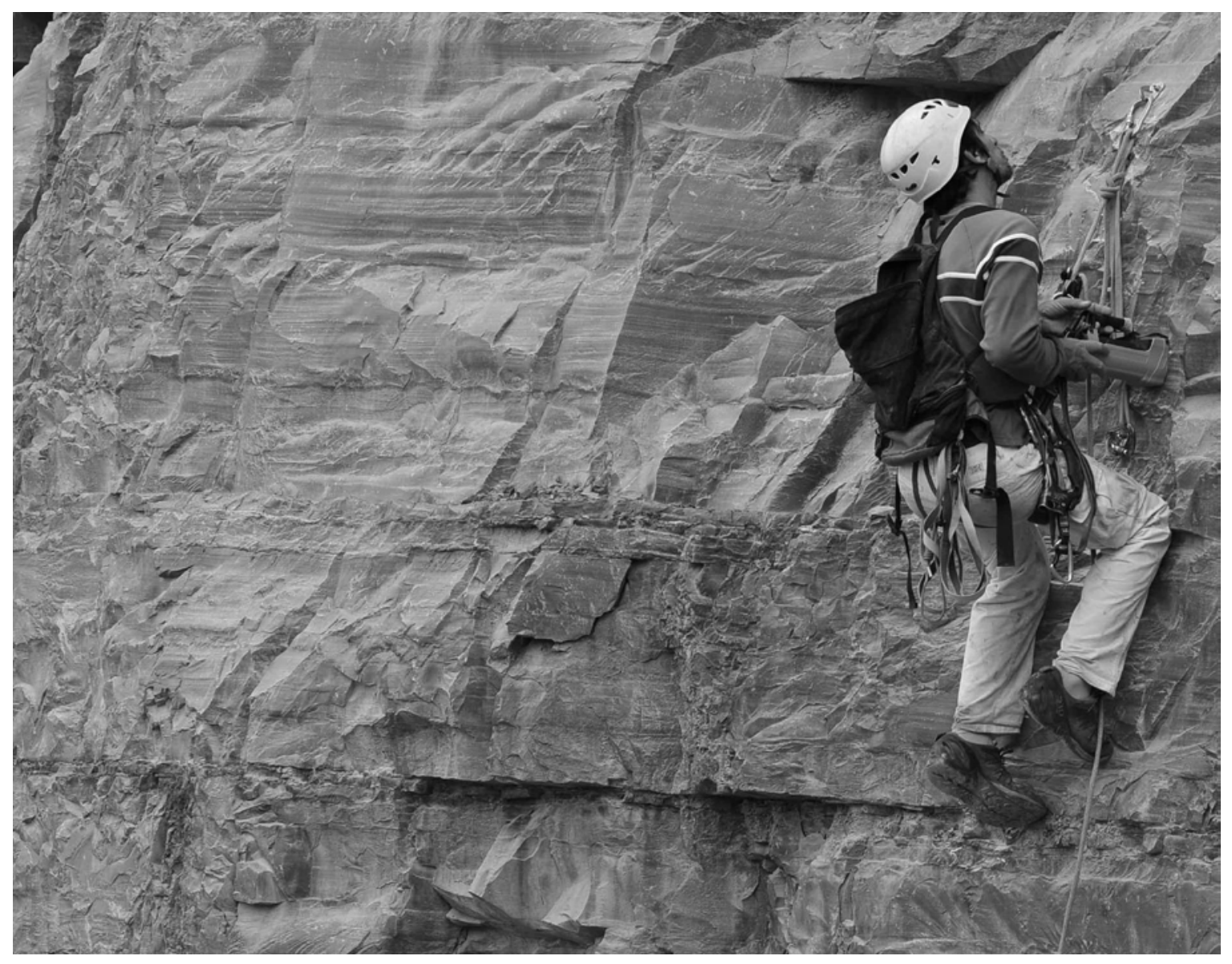

Figura 4. Utilização de técnicas verticais para acesso à rocha e aquisição de dados para construção dos perfis gama espectral. Pedreira Rio Preto, Palmeiras, BA.

\subsubsection{GEOQUÍMICA DE ROCHA TOTAL}

Análises geoquímicas quantitativas em rocha total foram obtidas através dos métodos laboratoriais convencionais ICP-ES (Inductively Coupled Plasma - Emission Spectroscopy) e ICP-MS (Inductively Coupled Plasma - Mass Sspectroscopy). Para quantificação do teor de carbono orgânico total (TOC - Total Organic Carbon), devido ao baixo limite de detecção, utilizou-se o método 2A LECO. 


\subsubsection{ESTRATIGRAFIA DE SEQUÊNCIAS DE ALTA RESOLUÇÃO}

O método Estratigrafia de Sequências de Alta Resolução (ESAR) consiste na aplicação dos conceitos de Estratigrafia de Sequência em afloramentos e testemunhos (AITKEN e HOWELL, 1996), sobretudo os que concernem à delimitação de sequências (sensu CATUNEANU et al., 2009; CATUNEANU et al., 2011). O método proposto em Strasser et al. (1999) norteou a hierarquização das diferentes sequências, descritas a seguir.

\section{SEquÊNCIA ELEMENTAR OU CICLO BÁSICO}

Corresponde à sequência com a menor espessura reconhecível em campo. Uma sequência elementar completa apresenta uma sucessão vertical de fácies que demonstra uma periodicidade dos fatores controladores da deposição e/ou crescimento microbial. Portanto, idealmente esta sucessão irá indicar um "raseamento" seguido de um "aprofundamento" da lâmina d'água. Estas tendências são verificadas em campo e delineadas a partir da representação gráfica dos Ciclos de Karagodin (KARAGODIN, 1975). Nesta representação, a base de um triângulo vermelho indica o ponto em que culmina a tendência do raseamento, por exemplo, no topo de uma fácies com estruturas que evidenciem exposição subaérea. 0 vértice de um triângulo azul indica o trecho associado ao "afogamento", momento de maior disponibilidade para o crescimento de determinado tipo de microbialito, por exemplo. Tipicamente a espessura de uma sequência elementar apresenta poucas dezenas de centímetros, raramente ultrapassa 1 metro, e o seu limite é marcado por superfícies de descontinuidade bem pronunciadas nos afloramentos. 


\section{SEquênCia de PEQUENA ESCALA}

Sequências de pequena escala são constituídas por sequências elementares. Em geral, a sua delimitação se dá a partir da identificação de trechos com maior espessura de determinada fácies que indique menor ou maior disponibilidade para implantação da fábrica carbonática, raseamento e afogamento, respectivamente, que foram estabelecidas anteriormente, durante a identificação da sequência elementar. Assim, o raseamento nestas sequências está vinculado ao trecho em que há espessura anômala da fácies definida anteriormente, durante a construção da sequência elementar, como indicadora de condições deposicionais com a menor lâmina d'água ou à ocorrência de fácies indicativas de exposição subaérea/feições diagenéticas. Em todos os casos, a descontinuidade verificada em afloramento é bastante pronunciada e por vezes mais nítida do que aquelas associadas às sequências elementares. O afogamento comumente está vinculado ao trecho com maior espessura da fácies definida na sequência elementar como indicativa de deposição sob condições com maior acomodação. Em campo, a representação de raseamento e afogamento foram realizadas com a utilização da simbologia dos Ciclos de Karagodin. A espessura de uma sequência de pequena escala normalmente varia de um a poucos metros, mas, raras vezes, pode apresentar algumas dezenas de centímetros.

\section{SEQUÊNCIA DE MÉdIA ESCALA}

Uma sequência de média escala é constituída por trechos com a ocorrência dominante de fácies de determinada associação de fácies em detrimento de outra, antagônicas em proximidade/distalidade, em relação à linha de costa. Esta sucessão geralmente se dá de forma brusca. Para as sequências de média escala, raseamento e afogamento foram melhor 
definidos a partir da integração dos dados litoestratigráficos e geofísicos, realizada no escritório. No campo as tendências de "rasear" ou "afogar" foram inferidas e representadas com a utilização da simbologia dos Ciclos de Karagodin. A espessura de uma sequência de média escala é métrica.

\subsubsection{ANÁLISES ISOTÓPICAS U/PB}

Análises isotópicas $\mathrm{U} / \mathrm{Pb}$ foram executadas com o equipamento SHRIMP II da Western Australia University e Universidade de São Paulo. Dois delgados níveis pelíticos, um com contribuição vulcanoclástica, foram coletados na base e trecho intermediário da Unidade B da Formação Salitre nas proximidades da Pedreira Rio Preto, município de Palmeiras. Zircões detríticos e magmáticos foram separados por meio de cominuição da rocha com britador e moinho de disco, separação de minerais pesados com líquidos densos (bromofórmio densidade $=2.85$ e diodo de metano - densidade $=3.32$ ), separação dos minerais menos magnéticos com separador isodinâmico e selecionados individualmente. Os grãos de zircões das amostras foram montados em seções com resina epóxi com padrões Temora e SL3 e polidas até exposição do interior dos grãos, os quais foram fotomicrografados com microscópio de luz refletida e transmitida e posteriromente imageados com uso de microscopia eletrônica de varredura (MEV) e catodoluminescência (CL). As seções com zircão foram limpas e cobertas com uma película fina de ouro para posterior análise dos isótopos de U-Pb com SHRIMP (Sensitive High-Resolution Ion MicroProbe).

Os métodos analíticos e tratamento de dados seguem aqueles propostos por Williams (1998) e Sato et al. (2014). Os grãos de zircão foram analisados com um feixe primário $\mathrm{O}_{2}$ com 2- 
3nA, $10 \mathrm{kV}$ e focalizado a um local (spot) de $\sim 30 \mu \mathrm{m}$ de diâmetro, com densidade do feixe de íons em torno de 2,5-7 nA (dependente da abertura de luminosidade). Todas as interferências dos isótopos de $\mathrm{Pb}$, Th e $\mathrm{U}$ foram retiradas por meio de análises com uma resolução de massa de 5000 a 5500. Redução dos dados e cálculos de idades foram realizadas com Squid 1.06 e Isoplot-Ex (LUDWIG, 2008). Concentrações foram determinadas por meio das medidas sistemáticas dos padrões SL 3 e idade de ${ }^{206} \mathrm{~Pb} / \mathrm{U}^{238}$ de referência foi determinada com uso do Temora 2.

\subsubsection{ANÁLISES ISOTÓPICAS DE C E O}

Técnicas isotópicas, sobretudo aquelas referentes à variação de isótopos estáveis como ${ }^{13} \mathrm{C}$, têm se mostrado ferramenta importante em pesquisas sobre paleoambientes e paleoclimas. De uso comum em sucessões carbonáticas do Neoproterozoico, estudos dessa natureza têm servido sobretudo para embasar discussões sobre as condições ambientais, à época da deposição, e para mensurar qualitativamente processos de alteração, diagenéticos ou não. $\mathrm{Na}$ pesquisa em curso, o resultado das análises foi utilizado para avaliar a assinatura isotópica e, tentativamente, associar a um intervalo da litoestratigrafia, já estabelecido a partir da assinatura isotópica (por exemplo, MISI et al., 2007).

As amostras foram coletadas a partir de espaçamentos quase sempre regulares, à exceção dos locais em que as condições de acesso e da rocha não permitiram coleta, e submetidas para análises após prévia triagem macroscópica, quando foram selecionadas apenas aquelas ais homogêneas - isentas de fraturas, alterações e/ou vênulas - e de composição essencialmente carbonática, sem a presença de terrígenos. 
Em fase posterior, nas dependências da UnB e no laboratório de preparação de amostras do Centro de Estudos em Petrologia e Geoquímica da UFRGS, o material pulverizado por amostra, cerca de 300 microgramas, foi obtido com auxílio de uma furadeira de bancada com broca $\varnothing 0,8 \mathrm{~mm}$, técnica de microdrilling, e submetido para aquisição das razões isotópicas. As análises foram realizadas no Laboratório de Isótopos Estáveis, no setor de Gases Nobres do Laboratório de Geocronologia da UnB. As razões isotópicas foram geradas a partir da reação entre a mistura gasosa de dióxido de carbono $\left(\mathrm{CO}_{2}\right)$ - proveniente da amostra - e ácido fosfórico $\left(\mathrm{H}_{3} \mathrm{PO}_{4}\right)$ concentrado a temperatura de $72^{\circ} \mathrm{C}$, em recipientes devidamente livres de gases atmosféricos.

O equipamento utilizado para obtenção desses dados foi o espectrômetro de massa Delta Plus Advange acoplado a Gas Bench II, com auto-sampler, projetado especialmente para receber amostras sob a forma de gás. As medidas das relações isotópicas são efetuadas em relação a um padrão secundário, adotado pelo laboratório, e posteriormente ajustado para estar conforme com um padrão internacional definido pela Agência Internacional de Energia Atômica (International Atomic Energy Agency - IAEA). Foram adotadas as diretrizes do padrão Vienna Pee Dee Belemnites (V-PDB), que admite um erro analítico de $\pm 0,1 \%$, para ambos os gases. Para obter as razões $\delta^{13} \mathrm{C}$ e $\delta^{18} \mathrm{O}$, o V-PDB (razão isotópica de uma concha Belemnite - Formação Pee Dee, Cretáceo, EUA) é comparado com as razões isotópicas de C $\left({ }^{13} \mathrm{C} /{ }^{12} \mathrm{C}\right)$ e $\mathrm{O}\left({ }^{18} \mathrm{O} /{ }^{16} \mathrm{O}\right)$, conforme as equações abaixo.

$$
\delta^{13} C=\left(\frac{{ }^{13} C /{ }^{12} C^{(\text {amostra })}}{{ }^{13} C /{ }^{12} C^{(\text {padrão })}}-1\right) 10^{3} \quad \text { e } \quad \delta^{18} O=\left(\frac{{ }^{18} O /{ }^{16} O^{(\text {amostra })}}{{ }^{18} O /{ }^{16} O^{(\text {padrão })}}-1\right) 10^{3}
$$




\subsubsection{Petrografia}

Estudos petrográficos foram empregados para determinar e caracterizar os constituintes das fácies e, quando possível, para qualificar os processos diagenéticos, e as suas relações temporais, e também para identificar o tipo de microporosidade. As lâminas delgadas foram confeccionadas no Laboratório de Laminação (LABLAM) do Instituto de Geociências da UnB e na Unidade de Operações de E\&P - Bacia de Santos, PETROBRAS.

As descrições das lâminas foram realizadas no Laboratório de Microscópia do Instituto de Geociências da Universidade Federal da Bahia (UFBA), com microscópio petrográfico modelo Olympus BX60, e no prédio da Pós-graduação em Estratigrafia da Universidade Federal do Rio Grande do Sul (UFRGS), com o microscópio petrográfico modelo Axio Imager 2 (Zeiss). As fotomicrografias digitais foram obtidas a partir dos microscópios citados - com câmera acoplada, apropriada para captura e processamento de imagens - e utilizando um estereomicroscópio modelo SteREO Discovery V12, acessado na PETROBRAS Unidade Operacional Bahia (UO/BA).

Dentre os procedimentos utilizados durante as descrições petrográficas, cabe mencionar que a identificação da dolomita foi realizada através do teste de coloração com Vermelho de Alizarina (Alizarina Red-S), em $\mathrm{HCl}$ a $2 \%$, e que e a porcentagem de cristais, aloquímicos e ortoquímicos foi estimada semiquantitativamente, utilizando gráficos visuais de comparação para estimar abundâncias dos diferentes constituintes (BACCELLE e BOSELLINI, 1965). A classificação de porosidade adotada é baseada em Choquette e Pray (1970) e em Tucker e Wright (1990) e o tamanho dos cristais de carbonato é citado conforme a tabela de 
cristalinidade disponibilizada em Folk (1980). Nas amostras com alto grau de neomorfismo utilizou-se a técnica do cartão branco - descrita em Folk (1987) - para detectar possíveis vestígios de aloquímicos. A classificação da rocha foi efetuada a partir das propostas de Folk (1959, 1962) e Dunham (1962), para rochas retrabalhadas, e Embry e Klovan (1971), Riding (2000), Riding (2002) e Terra et al. (2010), para rochas bioconstruídas. As principais informações obtidas por lâmina foram sintetizadas em folha de descrição apropriada.

\subsubsection{DIFRATOMETRIA POR RAIO X}

Análises por difração de Raio $X$ (DRX) foram realizadas em 6 amostras de rocha total coletadas em diferentes perfis. As análises DRX foram executadas no laboratório de DRX do Instituto de Geociências da UFRGS no difratômetro Siemens D5000. A preparação das amostras constou de moagem prévia e prensa em lâmina apropriada. Os softwares DECOMPXR $^{\circledR}$ e NEWMOD ${ }^{\circledR}$ (REYNOLDS, 1985) foram utilizados para promover uma caracterização mais aperfeiçoada dos componentes de argilominerais presentes nas amostras. 


\section{CONTEXTO GEOLÓGICO}

\subsection{Cratón do São Francisco}

A área de pesquisa está inserida na Província Estrutural do São Francisco, parte integrante da Plataforma Sul-americana (ALMEIDA et al., 1981). Esta província é composta essencialmente por uma região cratônica, denominada por Almeida (1967) de Cratón do São Francisco (CSF). A formação das principais unidades tectônicas que compõem o CSF ocorreu desde o Arqueano em resposta à aglutinação de uma imensa massa continental que amalgamou núcleos continentais distintos e gerou cinturões juvenis, retrabalhados especialmente no Paleoproterozoico (BARBOSA e SABATÉ, 2004).

Sucessivos ciclos tafrogenéticos e orogenéticos se sucederam e, durante a Orogênese Brasiliana (Ciclo Brasiliano 0.9 - 0.52 Ga; BRITO NEVES et al., 2014) -, o CSF é estabelecido nos moldes atuais, delimitado por terrenos criados ou deformados durante o Brasiliano, as "faixas móveis" brasilianas (Figura 5 A). Estes terrenos foram edificados em resposta aos esforços compressivos do referido ciclo orogenético que culminou com a formação do supercontinente Gondwana Ocidental (BRITO NEVES et al., 1999; ALKMIN et al., 2004).

Em área, o CSF abrange principalmente os estados de Minas Gerais e Bahia, e as "faixas móveis" que o circundam são assim denominadas: Brasília, limites sul e oeste, Rio Preto, noroeste, Sergipana e Riacho do Pontal, no trecho mais setentorial, e Araçuaí, limite sudeste (ALMEIDA et al., 1981) - Figura 5 B. Bacias sedimentares cretácicas, parcialmente submersas, compõem o limite leste, e têm criação associada à evolução da margem passiva que possibilitou a implantação do oceano Atlântico. 
A configuração regional do CSF é definida pelos domínios leste e oeste, separados pelo o domínio central, Aulácogeno de Paramirim (ALKMIM e MARTINS-NETO, 2012). O domínio central, onde está inserido o presente estudo (Figuras 5 B e C), constitui em um sistema de riftes paleo/meso e neoproterozoico parcialmente invertido e representa, na Bahia, o sítio deposicional dos supergrupos Espinhaço e São Francisco nas regiões da serra do Espinhaço Setentrional, vales dos rios Paramirim e São Francisco e na Chapada Diamantina (CRUZ, 2004; CRUZ e ALCKMIN, 2006).

Os supergrupos acima mencionados compreendem rochas metassedimentares e vulcânicas, depositadas entre o final do Paleoproterozoico e Neoproterozoico e, juntamente com rochas sedimentares depositadas durante o Fanerozoico, são designadas como "coberturas" do CSF. As "coberturas" estão sobre o embasamento cristalino, representado por granitóides do tipo TTG (tonalito-trondjemito-granodiorito) e sequências greenstone belts que constituem terrenos ígneos e metamórficos de grau médio a alto e têm idade $>1.8 \mathrm{Ga}$. Estes terrenos são mapeados como blocos Gavião, Jequié, Serrinha e Itabuna-Salvador-Curaçá (ALMEIDA, 1977; BARBOSA et al., 2004; CRUZ e ALKMIM, 2006; ALKMIM e MARTINS-NETO, 2012).

É possível ainda, na porção ocidental do Aulacógeno do Paramirim, individualizar uma zona de máxima inversão de orientação NNW-SSE denominada de "Corredor de Deformação do Paramirim" (ALKMIN et al., 1993), que é delimitada pela Falha de Santo Onofre, a oeste, e pelo lineamento João Correia - Barra do Mendes, a leste. Esta zona engloba parte do embasamento do CSF, além das unidades litoestratigráficas da cobertura, e apresenta uma deformação do tipo endodérmica. Falhas, inversas e de empurrão, e dobras são comuns, 
com dobras apresentando orientação preferencial para NNW e vergência para ENE. No trecho SW deste domínio a deformação é mais pronunciada e há o desenvolvimento de metamorfismo (GUIMARÃES et al., 2012).

A área do presente estudo está localizada a leste desse corredor de deformação, na região da Chapada Diamantina. Ali ocorrem principalmente as unidades pertencentes aos supergrupos Espinhaço e São Francisco e as estruturas mais evidentes são dobras abertas com orientação geral NNW-SSE a N-S (Figura 5 C). O embasamento do CSF não está envolvido na deformação, caracterizando assim uma deformação do tipo epidérmica (GUIMARÃES et al., 2012).

$\mathrm{Na}$ área de estudo o embasamento do CSF é representado por unidades do Bloco Gavião terrenos granito-gnáissicos e greesntone belts associados paleoarqueanos, neoarqueanos e paleoproterozoicos (MARINHO 1991; PEUCAT et al. 2002, BASTOS LEAL et al., 2003, BARBOSA E SABATÉ, 2004, BARBOSA et al., 2004, CRUZ et al., 2012, SANTOS-PINTO et al., 2012).

\subsection{Coberturas do Cratón do São Francisco}

\subsubsection{SUPERGRUPO ESPINHAÇO}

Na região da Chapada Diamantina, o Supergrupo Espinhaço é representado pelos grupos Rio dos Remédios, Paraguaçu e Chapada Diamantina que correspondem a uma sequência dominantemente siliciclástica, depositadas em condições intracratônicas durante o período compreendido entre $1.8 \mathrm{Ga}$ e $0.9 \mathrm{Ga}$. Recentemente estas unidades foram subdivididas em três sequências cronoestratigráficas por Guadagnin et al. (2015), correlacionáveis à outras, 
identificadas em diferentes regiões do CSF por Chemale et al. (2012); Santos et al. (2013) e Guadagnin e Chemale (2015).

\section{SEQUÊNCIA Estateriana (1,8-1,68 Ga; Espinhaço INfERIOR)}

A sequência Estateriana compreende o Grupo Rio dos Remédios. Este grupo é composto pelas formações Novo Horizonte, Lagoa de Dentro e Ouricuri do Ouro, que têm expressivos afloramentos na região sul da Chapada Diamantina. A primeira é constituída por litotipos vulcânicos de composição ácida e as duas últimas formações representam depósitos sedimentares relacionados a sistemas lacustres (Formação Lagoa de Dentro) e aluviais (Formação Ouricuri do Ouro). Vulcanismo de ca. 1,75 Ga data a deposição desta unidade. 


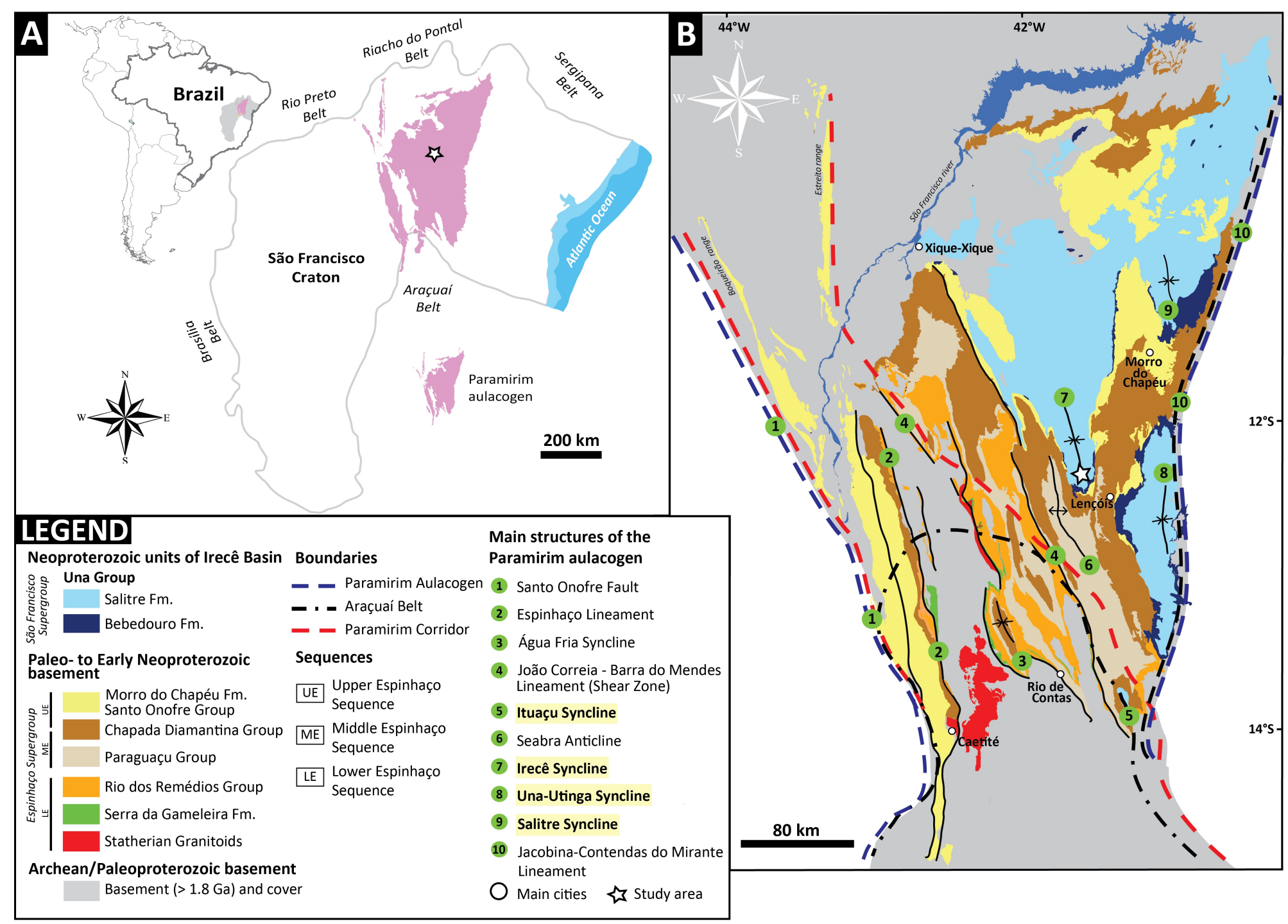

Figura 5. Contexto geológico simplificado do aulacógeno do Paramirim. Figura adaptada a partir de Alkmim et al (2006), Souza et al. (2003) e Guimarães et al. (2012). 


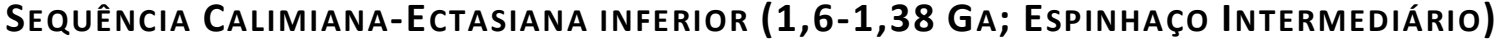

A sequência Calimiana-Ectasiana inferior (Espinhaço Intermediário) compreende o Grupo Paraguaçú (intrudido por diques de ca. 1,5 Ga) e a Formação Tombador, que possui zircões vulcânicos com idade de ca. 1,43 Ga. O Grupo Paraguaçu (DERBY, 1906), atualmente é dividido em duas unidades litoestratigráficas: Formação Mangabeira e Formação Açuruá Guimarães et al. $(2005,2008)$. A base do grupo é constituída por depósitos dominantemente eólicos, com alguma incursão fluvial, depositados em um sistema de erg costeiro, Formação Mangabeira, sucedidos por depósitos deltaicos e marinhos, no topo, Formação Açuruá.

A Formação Tombador consiste dominantemente em depósitos eólicos e fluviais efêmeros assentados diretamente sobre o embasamento cristalino na região norte da Chapada Diamantina. Ao sul, é representada por depósitos transicionais/continentais (flúvioestuarinos a aluvial/eólico) e o limite basal, que ocorre sobre a Formação Açuruá, é expresso por uma discordância angular regional (DERBY, 1906; PEDREIRA, 1994).

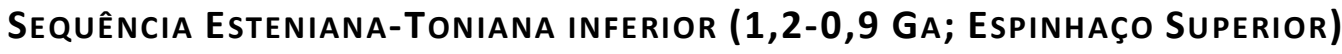

A sequência Esteniana-Toniana inferior compreende as formações Caboclo e Morro do Chapéu. A primeira equivale litoestratificamente a arenitos e pelitos, com conglomerados e carbonatos subordinados, interpretados como depósitos flúvio-estuarinos que passam, em direção ao topo, gradativamente para depósitos marinhos rasos. A Formação Morro do Chapéu é interpretada como depósitos estuarinos, com ação fluvial e de maré, e eólicos (GUIMARÃES et al., 2012). 


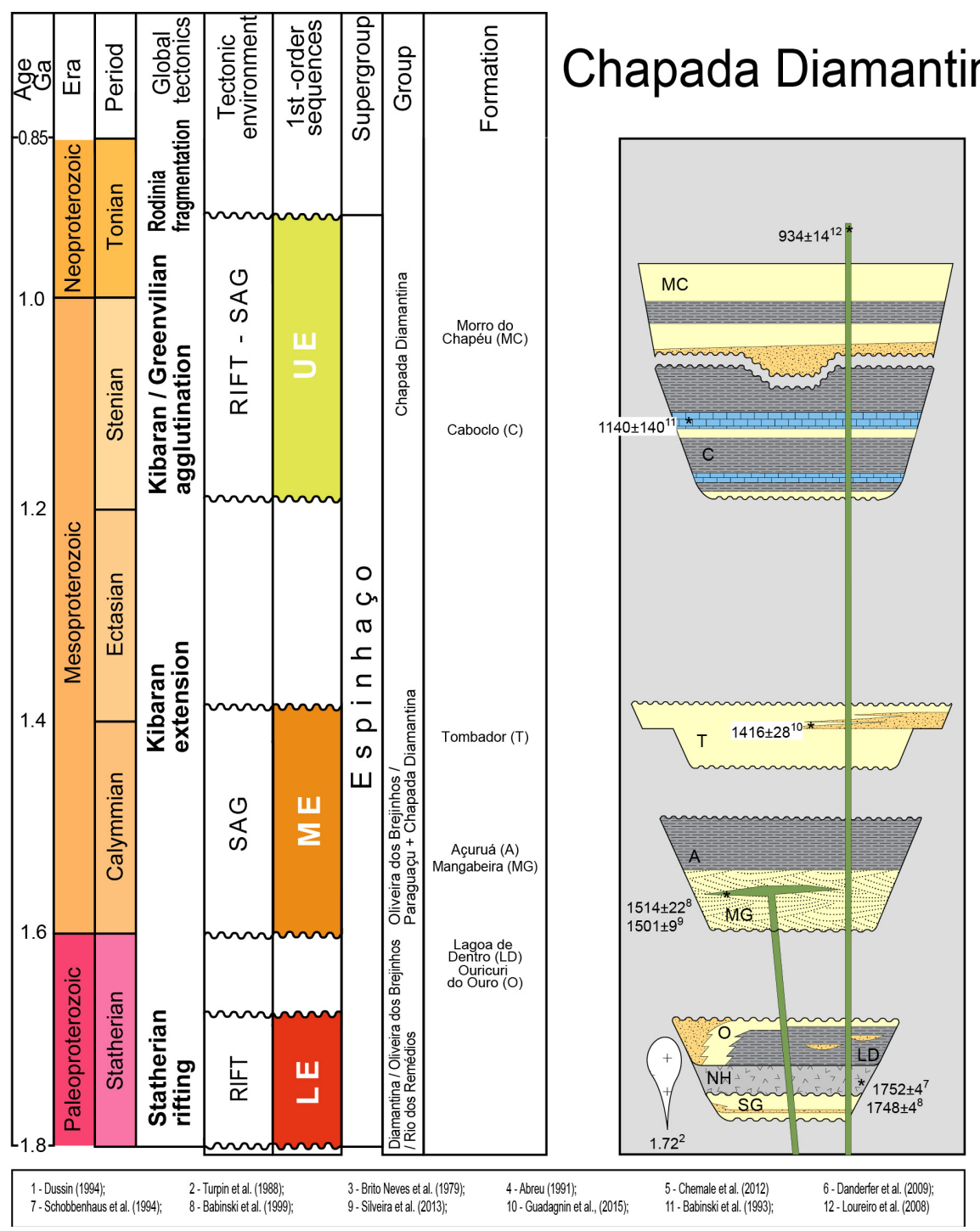

Figura 6. Coluna estratigráfica com as sequências do Supergrupo Espinhaço na região da Chapada Diamantina. Extraída de Guadagnin et al. (2015b). 


\subsubsection{SUPERGRUPO SÃO FRANCISCO}

O Supergrupo São Francisco, na região de estudo, é constituído pelas formações Bebedouro e Salitre. Está em discordância erosiva de caráter regional sobre as rochas do embasamento do CSF e do Supergrupo Espinhaço. Por haver um hiato deposicional (discordância erosiva e significativa lacuna temporal), além de uma diferença significativa entre seus litotipos devido à variação paleoclimática ocorrida entre a deposição das diferentes formações, Guimarães et al. (2012) propuseram tratar as formações de forma individual e não em única unidade litoestratigráfica (Grupo Una; PEDREIRA et al. 1985). Além das justificativas acima, os autores se basearam na separação já efetuada em unidades litoestratigráficas cronocorrelatas, no estado de Minas Gerais e na região oeste da Bahia.

Em termos cronoestratigráficos, as formações Bebedouro e Salitre equivalem, respectivamente, às sequências Macaúbas e Bambuí (ALKMIN e MARTINS-NETO, 2012). As exposições destas formações ocorrem exclusivamente na região da Chapada Diamantina em sinclinais denominadas de Irecê, Ituaçu, Salitre e Una-Utinga, comumente referidas na literatura como "bacias" ou "sub-bacias". Na presente tese, Bacia de Irecê irá se referir à exposição do Supergrupo São Francisco na sinclinal homônima.

A coluna estratigráfica simplificada do Supergrupo São Francisco na região da Chapada Diamantina pode ser visualizada na Figura 7. 


\begin{tabular}{|c|c|c|c|c|c|c|c|c|}
\hline \multirow{2}{*}{ 을 } & \multicolumn{3}{|c|}{$\begin{array}{c}\text { Cronoestratigrafia } \\
\text { (Cohen et al., 2013) }\end{array}$} & \multicolumn{5}{|c|}{ Carta Estratigráfica Simplificada - Supergrupo São Francisco - Bacia de Irecê, BA } \\
\hline & ÉON & ERA & PERÍODO & $\begin{array}{c}\text { Supergrupo/ } \\
\text { Grupo }\end{array}$ & Formação & $\begin{array}{c}\text { Dados } \\
\text { Geocronológicos }\end{array}$ & $\begin{array}{l}\text { Seção } \\
\text { Estratigráfica } \\
\text { Simplificada }\end{array}$ & $\begin{array}{c}\text { Sistemas } \\
\text { Deposicionais }\end{array}$ \\
\hline 0.6 & \multirow{3}{*}{ 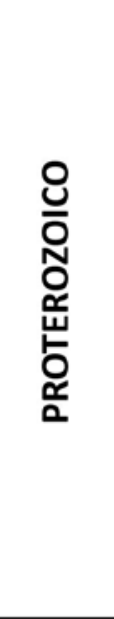 } & \multirow{3}{*}{ 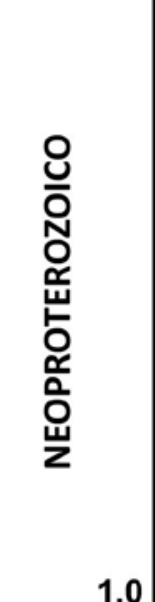 } & $\begin{array}{ll}\text { Ediacarano } & \\
& 0,63 \\
\end{array}$ & & & \multirow{3}{*}{$\begin{array}{l}\text { ca. } 669 \mathrm{Ma}(\mathrm{U} / \mathrm{Pb})^{*} \\
\text { ca. } 874 \mathrm{Ma}(\mathrm{U} / \mathrm{Pb})^{* *}\end{array}$} & \multirow{3}{*}{ 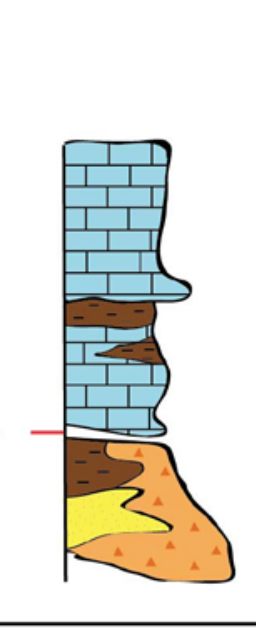 } & \\
\hline $\begin{array}{l}0.7- \\
0.8-\end{array}$ & & & Criogeniano & \multirow{2}{*}{ 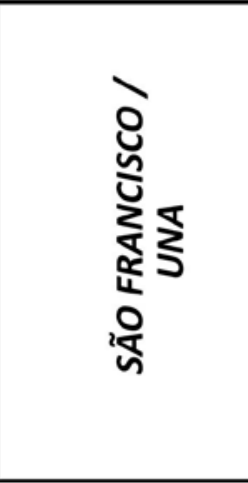 } & Salitre & & & $\begin{array}{l}\text { Carbonático } \\
\text { Marinho }\end{array}$ \\
\hline 0.9 & & & Toniano & & Bebedouro & & & $\begin{array}{l}\text { Glacial- } \\
\text { marinho }\end{array}$ \\
\hline
\end{tabular}

Figura 7. Coluna estratigráfica simplificada do Supergrupo São Francisco na região da Chapada Diamantina. Dados geocronológicos obtidos em Santana et al. (submetido)* e Figueiredo (2008)**, e significam idades máximas de deposição. 


\section{SEQUÊNCIA MaCaúbas (CRIOGENIANo INFERIOR)}

$\mathrm{Na}$ Chapada Diamantina, esta sequência equivale à Formação Bebedouro (OLIVEIRA e LEONARDOS, 1943). De acordo com Guimarães (1996) e Figueiredo (2008), esta formação fora depositada em um contexto glacio-marinho proximal, ou proglacial, associado ao derretimento de icebergs, e em contexto continental extraglacial. Diamictitos, arenitos e pelitos representam os principais depósitos dessa formação, sendo que os primeiros são os litotipos com maior expressão areal. Nos níveis pelíticos são relatados seixos e clastos deformando a laminação (dropstones). Arenitos têm ocorrência mais subordinada, compreendendo corpos, em geral, pouco espessos e de composição diversificada (subarcóseos, arcóseos, grauvacas e quartzo-arenitos). A Formação Bebedouro possui espessura máxima de 100 m. Zircões detríticos de ca. 874 Ma indicam a idade máxima de deposição (FIGUEIREDO, 2008).

\section{Sequência bambuí (CrIogeniano médio a superior)}

Esta sequência é representada pela Formação Salitre, composta por depósitos de ambiente marinho plataformal (MISI, 1979; BOMFIM et al., 1985; SOUZA et al., 1993). É constituída por rochas carbonáticas, calcíticas e dolomíticas, com pequenas intercalações de rochas terrígenas, e tem como principais litotipos calcilutitos, calcissiltitos, dolo/calcarenitos, além de rochas biocontruídas. Formação Salitre foi subdividada em unidades informais, e as proposições dos diferentes autores estão descritas no próximo capítulo. A base da Formação Salitre é marcada por discordância erosiva e angular no contato com rochas do embasamento, do Grupo Chapada Diamantina ou, mais comumente, com as rochas da Fm. Bebedouro. No topo, é recoberta por formações superficiais cenozoicas. Possui espessura de, no máximo, $500 \mathrm{~m}$. Quanto à idade de deposição, dados recentes obtidos em zircões de 
rocha com contribuição vulcanoclástica pela técnica SHRIMP de análises isotópicas U/Pb indicam uma idade máxima de $669 \mathrm{Ma}$ (+/- $14 \mathrm{Ma}$ ) - Santana et al. (submetido). Estes dados são inéditos e resultam da presente pesquisa.

\subsection{SíNTESE dA EVOLUÇÃo TECTÔNICA}

Uma síntese sobre a evolução tectônica do CSF é realizada com ênfase na implantação das bacias sucessoras que ocorrem na região da Chapada Diamantina. De acordo com Barbosa e Sabaté (2004), os dados mais antigos do CSF sugerem que no Paleoproterozoico, por volta de 2.8-2.6 Ga, ocorreu a aglutinação de uma imensa massa continental arqueana. Esta aglutinação envolveu núcleos continentais distintos, com idade de até 3.4 Ga. No Sideriano há registros de uma trafogênese responsável por romper essa aglutinação, em torno de 2.5 Ga. Uma nova junção ocorre com a orogênese Transamazônica (2.3-2.15 Ga), responsável por unir os blocos Gavião, Jequié, Serrinha e Itabuna-Salvador-Curaçá. Um significativo evento de rompimento acontece no Estateriano (1.8 -1.6 Ga) e proporciona a criação do sistema de riftes em que se desenvolve o vulcanismo Rio dos Remédios. A fase sinéclise é implantada no Calimiano, quando há a deposição do Supergrupo Espinhaço em uma bacia do tipo rifte-sag. A fase sag é representada pelos depósitos eólicos da Formação Mangabeira e marinhos rasos da Formação Açuruá, Grupo Paraguaçu (GUIMARÃES et al., 2008).

Após a trafogênese Estateriana, outros eventos se sucederam. O primeiro destes eventos, há cerca de 1.6 Ga, foi responsável por formar uma bacia do tipo sinéclise que extravasou os limites do rifte. Esta subsidência decorreu de um regime termo-flexural e possibilitou a criação de espaço para deposição das rochas que compõem o Grupo Chapada Diamantina (Sequência Tombador-Caboclo; Dominguez, 1993; Scherer et al., 2013). Entre os períodos 
Toniano e Criogeniano ocorre um importante evento distensional, trafogênese Toniana, que proporcionou a reativação de estruturas riftes/sinéclises pré-existentes. O supergrupo São Francisco é depositado nestas "calhas". A orogênese Brasiliana é caracterizada por importantes suturas que margeam o CSF, registros das colisões - margem ativa - e fechamento da margem passiva, que unificaram o trecho oeste do supercontinente Gondwana (Figura 13).

Conforme sugerido por Dominguez (1993), a acumulação dos sedimentos carbonáticos do CSF ocorre em uma ampla sinéclise intracratônica que, nas proximidades dos orógenos brasilianos, como na região sul do CSF, torna-se uma bacia de ante-país e atua como sítio receptor de sedimentos provenientes das faixas de dobramentos que delimitam o CSF. 


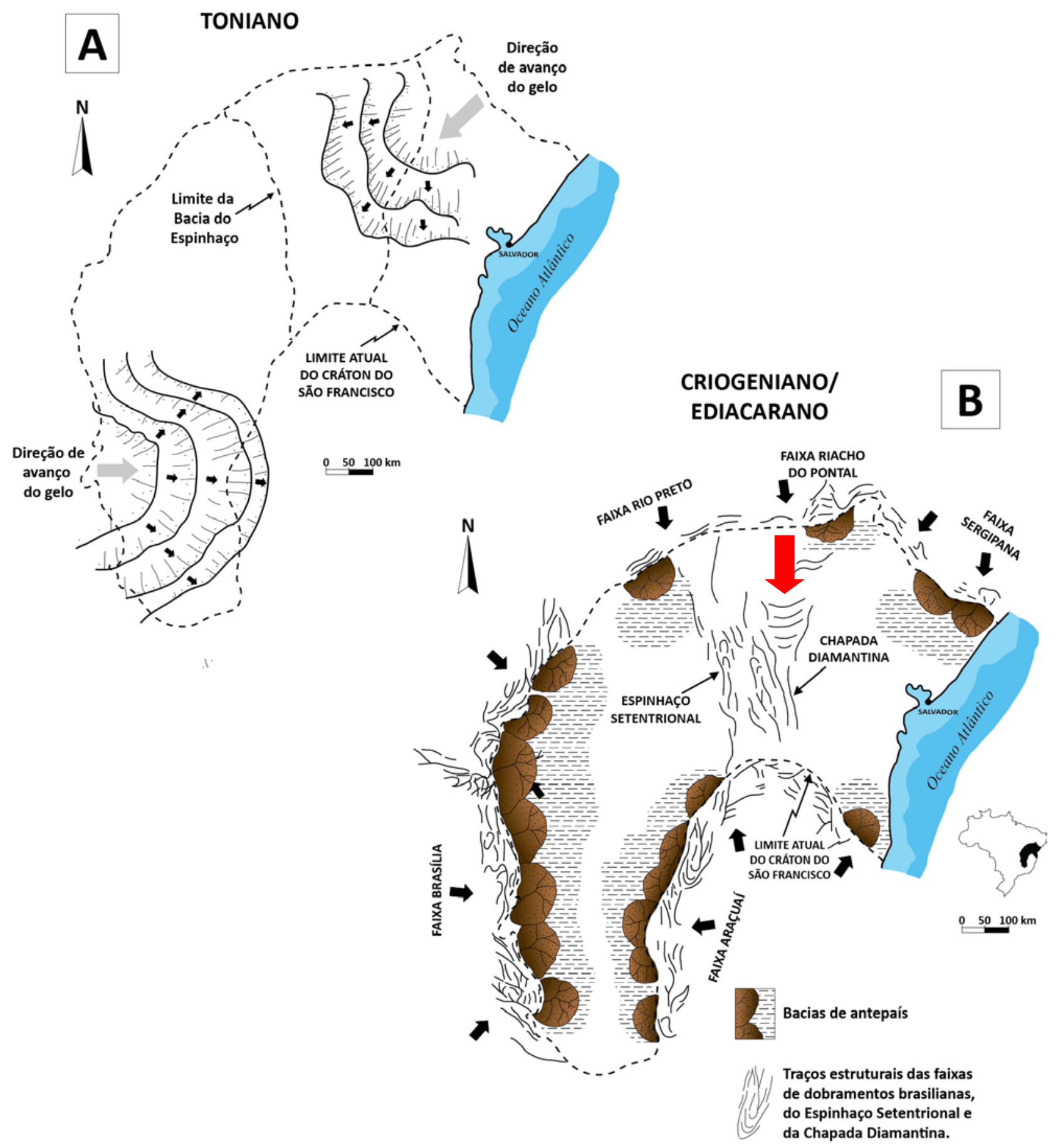

Figura 8. A trafogênese Toniana proporcionou a reativação de estruturas pré-existentes e gerou sítios deposicionais para a implantação do Supergrupo São Francisco (SSF). A - Direção geral do avanço de geleiras responsáveis pela sedimentação glácio-marinha da base do SSF. B - Destaque para as importantes suturas que margeam o cráton São Francisco e principais trechos com implantação de bacias de antepaís (modificado de DOMINGUEZ, 1993). A Bacia de Irecê, seta vermelha, representa um cinturão epidérmico de antepaís (REIS et al., 2013). 


\section{FORMAÇÃO SALITRE - EVOLUÇÃO DO CONHECIMENTO}

As primeiras expedições científicas ao interior da Bahia, século XIX, descrevem e reconhecem a importância das rochas carbonáticas na constituição geológica do estado. Relatos de viagem, como o da emblemática Expedição Thayer (1865-66), são reportados no livro Physical Geography and Geology of Brazil (HARTT, 1870) e reúnem as primeiras descrições geológicas realizadas na área da tese e cercanias.

Entre as diversas narrativas comuns à época, destacam-se o relato de viagem do naturalista Joel Asaph Allen, realizado em 1868 e referido em Hartt (1870) e em Derby (1906), e o relatório de viagem de Theodoro Sampaio - Sampaio (1880) apud Derby (1880) -, por apresentarem os principais aspectos litológicos e estruturais do que mais tarde seria denominado por Branner (1911) de "Série Calcário Salitre". Branner (1911) reconhece uma importante discordância, angular e erosiva, entre essa série e as rochas subjacentes.

A extensão em área de ocorrência e a exuberância dos afloramentos associados à "série" constam em diversas publicações do início do Século XX (DERBY 1905, 1906; BRANNER, 1911). Derby (1906), por exemplo, descreve "magnífica escarpa de calcário plaqueado azul claro, com cerca de $40 \mathrm{~m}$ de altura. Algumas das camadas estão cheias de nódulos de sílex que, com o intemperismo, garantem uma maneira de acompanhá-las..." em referência às escarpas do rio Una, Chapada Diamantina centro-oriental. 
Consta em Branner (1911) a primeira alusão à origem orgânica e ao possível contexto paleoambiental para formação dos grãos que compõem o "Calcário Salitre". A partir da opinião de consultores, Branner (1911) sugere que a deposição da rocha ocorreu em ambiente marinho raso, devido à predominância de grão aloquímico do tipo oólito, simples e composto, nas amostras analisadas.

Estudos sobre as rochas carbonáticas que afloram na região centro-norte da Bahia prosseguem com forte caráter descritivo (por exemplo, WILLIAMS, 1930) e um melhor entendimento sobre o processo de geração dessas rochas advém somente nos primeiros anos da década de 1970, a partir dos trabalhos de empresas públicas e privadas, muitas vezes associadas com universidades. Estes trabalhos tiveram como foco a metalogênese das ocorrências e depósitos de fosfato e sulfetos de metais-base, e serão mencionados mais adiante.

Oliveira e Leonardos (1943) adotam o nome "Série São Francisco Bambuí" e Formação Una para designar as rochas carbonáticas da região central da Bahia, modificado do termo "Série Bambuí" originalmente adotado em Rimann (1917) para englobar as rochas pelíticas e carbonáticas que afloram nas cercanias do município de Bambuí, Minas Gerais. Devido à similitude das fácies descritas em ambos estados, Oliveira e Leonardos (1943) sugerem uma possível ligação entre os sítios deposicionais das diferentes áreas.

Os trabalhos sucessivos têm entendimento análogo; adotam na área de estudo os mesmo termos definidos em Minas Gerais - divisão litoestratigráfica sugerida por Costa e Branco (1961), adaptada e uniformizada em Dardenne (1978). Somente em Renger (1973) há uma 
quebra na hegemonia desta abordagem. Este autor sugere que o termo "Grupo Bambuí" seja utilizado apenas na Bacia do São Francisco, Minas Gerais e oeste da Bahia, e que nas demais, com sequências carbonáticas cronocorrelatas, seja utilizado o termo "Supergrupo São Francisco".

Entre os trabalhos de caráter regional realizados na década de 70 , destacam-se os projetos Chumbo-Zinco no Bambuí (MISI 1970, 1973), Depósitos Minerais da Chapada Diamantina (MISI, 1975) e Bahia (PEDREIRA et al., 1975). O Projeto Bahia adota o termo "Supergrupo São Francisco", conforme sugerido por Renger (1973) e, nas bacias dos rios Verde, Jacaré e Una, insere o termo Grupo Una, subdividindo-o em duas formações: Salitre - que corresponde às fácies carbonáticas anteriormente referidas como Formação Sete Lagoas - , e Bebedouro - que corresponde às fácies glaciogênicas.

Para Pedreira et al. (1975), a adoção do termo Grupo Una ratifica a ideia que houve um total isolamento entre as bacias do São Francisco e Irecê. Para a Formação Salitre, no entorno da Fazenda Boa Vista, vale do rio Jacaré, esses autores individualizam 6 níveis litoestratigráficos (a, b, c, d, e e f) baseados sobretudo na diferença composicional e ocorrências minerais dos diferentes níveis. Devido à ausência de caracteres distinguíveis em imagens e fotografias aéreas, e também à escala do trabalho, estes níveis não foram representados em mapa. A presença de estromatólito na unidade superior, f, sugere deposição em ambiente com águas rasas e temperatura amena.

Consta em Misi e Souto (1975) a primeira subdivisão litoestratigráfica que delimita unidades informais mapeáveis em escala regional. Entretanto, coube à Misi (1979) congregar o quê, à 
sua época, havia de referências sobre sedimentologia e estratigrafia do Grupo Bambuí na Bahia - trabalhos de Misi (1970, 1973, 1975, 1976, 1978), Misi e Souto (1975) e Torquato e Misi (1977). Este significativo trabalho de síntese estabelece a primeira coluna estratigráfica com ciclos associados. As unidades informais foram nomeadas por letras C, B, B1, A e A1 e são descritas abaixo, de forma resumida, a partir dos trabalhos de Misi (1979), Misi e Kyle (1994) e Misi e Silva (1996).

\section{UNIDADE C}

A unidade C é constituída por dolomitos e calcários dolomíticos argilosos, vermelhos, comumente intercalados com níveis milimétricos a centimétricos de metargilito, verde ou vermelho. Eventualmente há presença de laminações microbiais, estromatólitos e gretas de ressecamento, sobretudo na base da unidade. Há ocorrência de minerais oxidados de cobre, disseminados ou preenchendo microfraturas. A deposição dessa unidade primeiramente foi associada à sedimentação em ambiente lacustre (TORQUATO e MISI, 1977). Entretanto, atualmente é consensual interpretar essa unidade como resultado de deposição em um contexto marinho restrito, implantado pós-glaciação da Formação Bebedouro (por exemplo, GUIMARÃES et al., 2012). A unidade C tem expressiva e contínua área de ocorrência na borda leste da Bacia de Irecê. Na borda oeste, a mesma ocorre de forma descontínua. A espessura é bastante variável, sendo a máxima registrada de $60 \mathrm{~m}$.

\section{UNIDADE B}

A unidade B é composta por calcários, por vezes dolomíticos, cinza-claros, laminados e/ou interestratratificado com leitos de metargilito, podendo também ocorrer intercalações com 
calcário preto, oolítico, e pseudomorfos de gipsita preenchidos por quartzo microcristalino, sobretudo no topo.

Essa unidade é interpretada como registro de uma sedimentação relativamente profunda, na base, com evidências de que, para o topo, há uma tendência regressiva (intermaré), constituindo assim uma sequência marinha do tipo raseamento para o topo (shallowing upward). A unidade B apresenta boas exposições em toda bacia, com espessura variando entre 100 a $200 \mathrm{~m}$.

\section{UNIDADE B1}

A unidade B1 é representada por dolomitos silicosos, dolarenitos (silicosos e oolíticos) e dololutitos, cinza-claros, com nódulos e lentes de sílica e de calcita. Ocorrem estruturas sedimentares como tepee e brechas intraformacionais lamelares, que caracterizam exposição subaérea em clima árido, além de laminações estromatolíticas. Podem ocorrer também corpos lenticulares e de espessuras métricas de calcários pretos, ricos em matéria orgânica, oolíticos, e parcialmente dolomitizados. A unidade B1 contém as principais ocorrências de $\mathrm{Pb}$ - Zn e fosfato da Bacia Irecê. Barita e fluorita também ocorrem, de forma subordinada.

De acordo com os trabalhos compilados, Misi (1979), Misi e Kyle (1994) e Misi e Silva (1996), a deposição dessa unidade ocorreu em provável ambiente árido, com exposição subaérea, em um contexto de planície de maré. A unidade B1 aflora em toda bacia, sobretudo na borda oeste e na região sul, sempre de forma descontínua e com as exposições apresentando espessura métrica. 


\section{UNIDADE A}

A unidade A é caracterizada por siltitos, argilitos calcíticos e margas, cinza-escuros - quando não alterados -, cinza-claros ou avermelhados, quando alterados. Estes litotipos exibem pronunciada laminação. Agregados de cristais cúbicos de pirita podem ocorrer. Misi e Silva (1996) sugerem que a deposição dessa unidade ocorreu em ambiente marinho, com lâmina d'água mais profunda, em relação às demais unidades. As melhores exposições ocorrem na borda oeste da bacia. A espessura dessa unidade varia de poucos metros a $100 \mathrm{~m}$.

\section{UNIDADE A1}

A unidade A1 é composta por calcilutitos e calcários oolíticos e pisolíticos, ricos em matéria orgânica. Estratificação cruzada e níveis ricos em intraclastos são comuns e interpretados como produtos de processos verificados em ambiente com lâmina d' água rasa, agitado. Esta unidade está muito bem representada na Bacia de Irecê, principalmente a norte da cidade homônima. A espessura é estimada em mais de $150 \mathrm{~m}$.

Com exceção da unidade informal C, as demais dispostas verticalmente compõem 2 ciclos de raseamento ascedente (Shallowing-upward carbonate cycles) da ordem de centenas de metros de espessura, correlacionáveis em escala bacial - Figura 9. Flint (1979) também identifica dois grandes ciclos de sedimentação, associando-os à correlação litoestratigráfica proposta em Dardenne (1978) para o Supergrupo São Francisco. 
Nova subdivisão litoestratigráfica para Formação Salitre é proposta no relatório final do Projeto Irecê/CPRM (BOMFIM et al. 1985) baseada em mapeamento geológico na escala 1:60.000 na região de entorno da cidade homônima - Figura 10. Outros projetos mapearam diferentes regiões da bacia - Pedreira et al. (1987); Monteiro et al. (1987) e Souza et al. (1988) - e foram responsáveis por subdividir a Formação Salitre em diferentes unidades e subunidades a partir de características faciológicas.

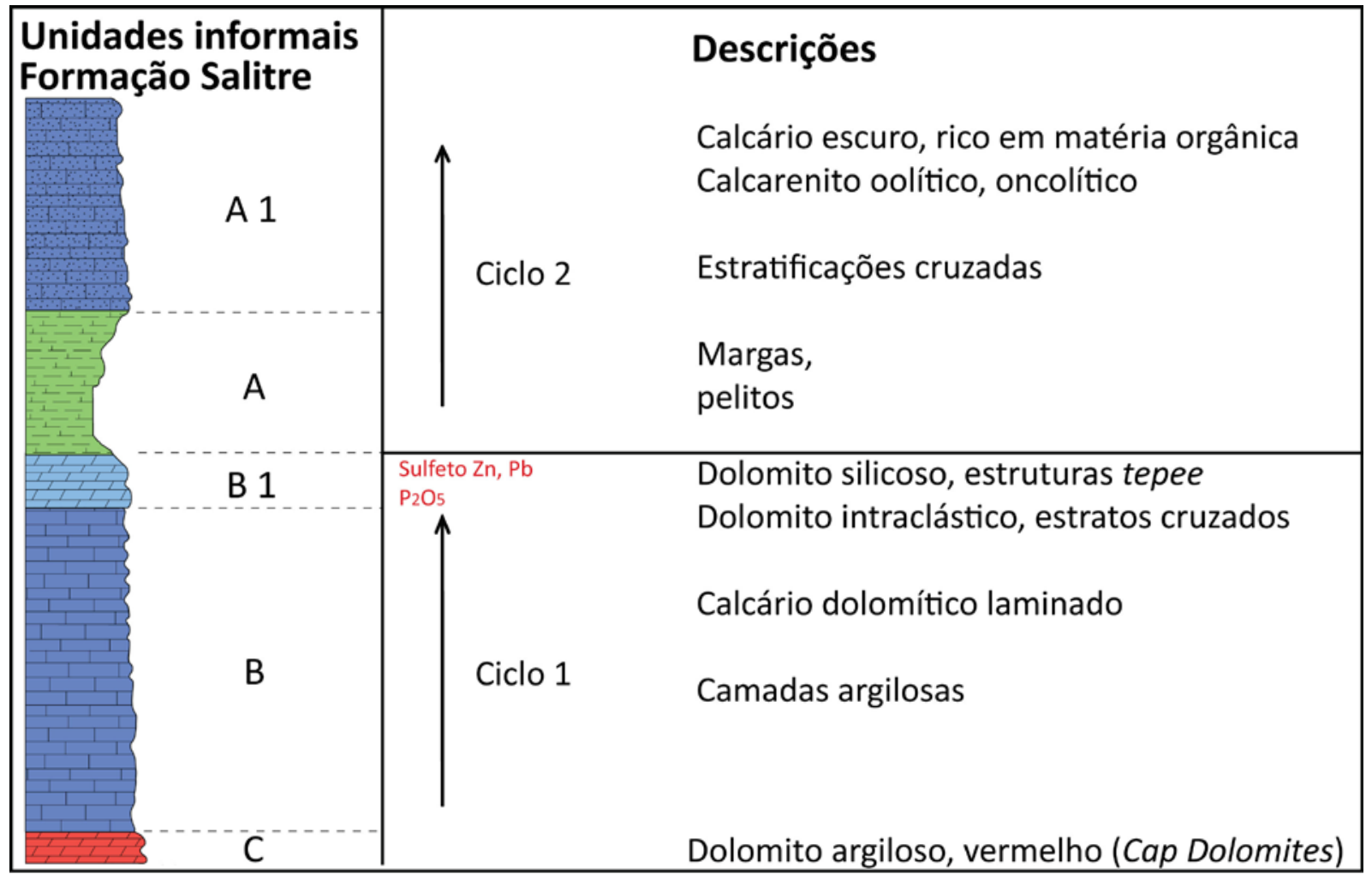

Figura 9. Unidades informais definidas para Formação Salitre na Bacia de Irecê com ciclos de raseamento ascedente identificados, de acordo com Misi (1979) e Misi e Kyle (1994). Figura adaptada a partir de Misi e Silva (1996). 
Souza et al. (1993), estudando a Bacia de Irecê na sinclinal homônima, revisaram a litoestratigrafia e subdividiram a Formação Salitre em unidades e subunidades informais (Figura 11), em parte anteriormente propostas em Bomfim et al. (1985), descritas a seguir.

\section{UNIDADE NOVA AMÉRICA}

A unidade Nova América tem ampla distribuição areal na Bacia de Irecê. Equivale às unidades Nova América e Gabriel, além da Fácies Lapão, discriminadas em Bomfim et al. (1985) e em Pedreira et al. (1987), e às unidades Cafarnaum, América Dourada e Soares, descritas em Souza et al. (1988). É subdividida em Nova América Inferior e Nova América Superior. A primeira é formada por dois litotipos principais: calcissiltitos, por vezes com estratificação ondulada e truncada, e laminitos algais, francamente ondulados e, por vezes, com estruturas que evidenciam exposição subaérea, tais como tepees associados a nódulos de calcita, além de bird's eyes. Localmente nota-se a presença de calcário dolomítico rosaavermelhado e estromatólitos, sobretudo na base da unidade. Também são descritos calcarenitos finos, dolarenitos e brechas.

A subunidade Nova América Superior é caracterizada por uma alternância de bancos carbonáticos. Da base para o topo, esses bancos correspondem a dolarenitos finos a dolossiltitos, com estratificação cruzada, dolarenitos oolíticos e oncolítos, por vezes com estratificação gradacional e ondulações truncadas (hummocky cross-stratification), e dolorruditos. São relatadas ocorrências de fosfato, bem como gossans com altos teores de chumbo e de zinco. 
A subunidade Nova América Inferior tem sido associada a depósitos característicos de ambiente de inter a supramaré. Os nódulos de calcita associados aos tepees são interpretados como pseudomorfos de gipsita, o que indica a prevalência de clima hiperárido durante a deposição - Souza et al. (1993). Para a subunidade Nova América Superior, as estruturas descritas sugerem deposição em ambiente com mais alta energia.

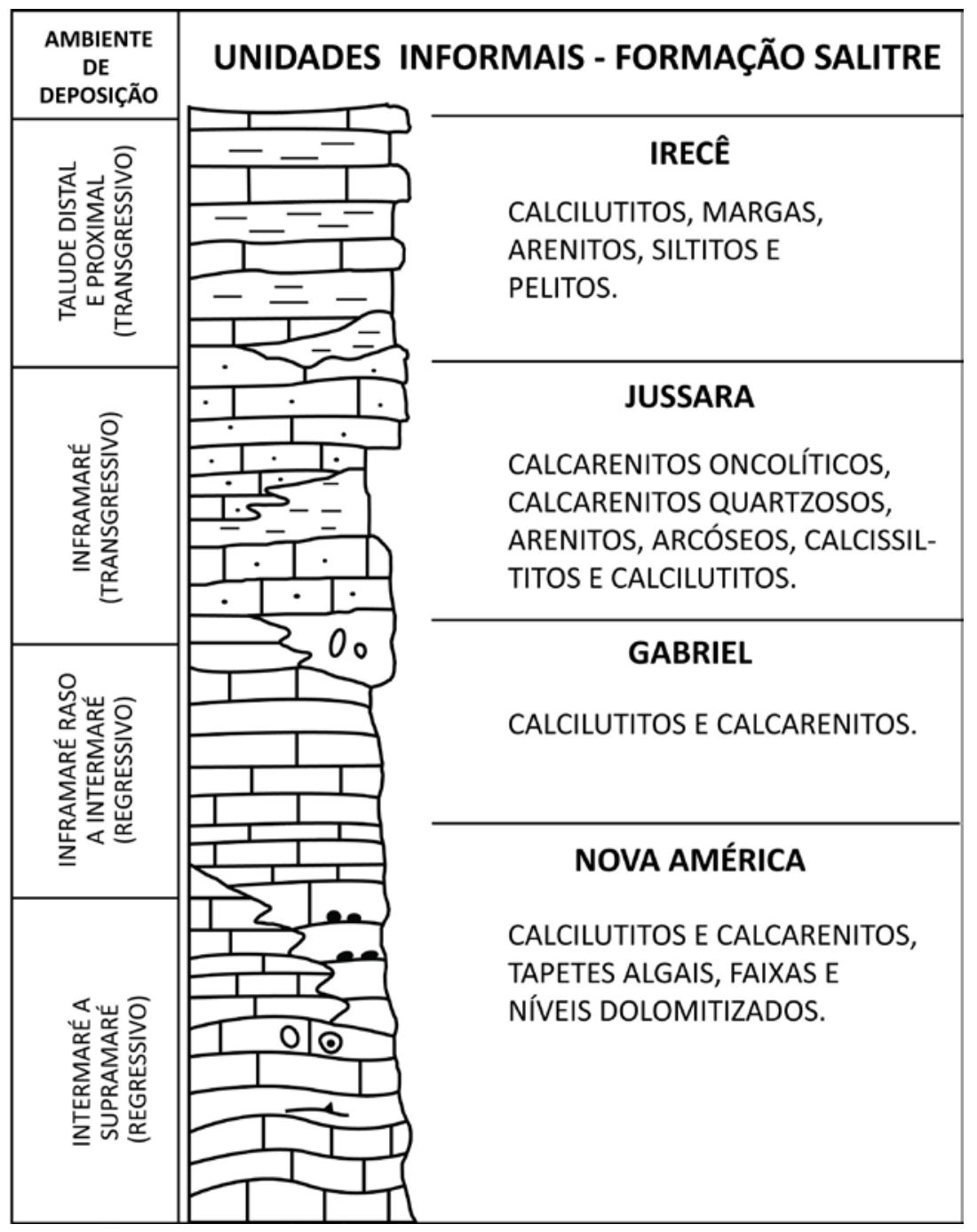

Figura 10. Unidades informais propostas para a Formação Salitre por Bomfim et al. (1985) e Pedreira et al. (1987). Figura adaptada a partir de Lagoeiro (1990). 


\section{SUBUNIDADE SARANDI}

A subunidade Sarandi (BOMFIM et al., 1985; PEDREIRA et al., 1987) tem ocorrência restrita à parte central da bacia. É composta pela alternância entre calcissiltitos e calcarenitos peloidais, por vezes contendo oncólitos e intraclastos. Apresenta dolomitização incipiente e estratificações do tipo cruzada e plano-paralela, além de marcas de carga. É interpretada como depósitos em ambiente de inframaré. Souza et al. (1993) sugerem que esta subunidade seja o correspondente distal da subunidade Nova América Superior ou o proximal da Unidade Jussara, descrita a seguir.

\section{UNIDADE JUSSARA}

A unidade Jussara ocorre em áreas restritas à porção mais central da Bacia de Irecê. As diferentes fácies que a caracterizam foram descritas por Bomfim et al. (1985), Pedreira et al. 1987 e Souza et al. (1988). Souza et al. (1993) subdividiram esta unidade em Jussara Inferior e Superior. A subunidade Jussara Inferior é formada por calcissiltitos, calcilutitos, calcarenitos - oolíticos e oncolíticos - e por calcarenitos com concreções esferoidais, além de calcarenitos quartzosos e arenitos arcosianos. A subunidade Jussara Superior é caracterizada por calcarenitos oolíticos e/ou oncolítico, com granulação que varia de fina a grossa. Estes calcarenitos geralmente são maciços, têm forte odor característico e estão dispostos em forma de bancos, sendo por vezes intercalados com calcissiltitos. Localmente há ocorrência de estromatólitos colunares e de trombólitos. A unidade é interpretada como depósitos de inter a inframaré (SOUZA et al., 1993). 


\section{UNIDADE IRECÊ}

A unidade Irecê apresenta maior concentração de afloramentos na região centro-oeste da bacia. É composta pela alternância entre litotipos carbonáticos (calcarenitos finos e calcilutitos, laminados) e terrígenos (margas, siltitos e arenitos imaturos). Bomfim et al. (1985) interpretam que esta unidade consiste em depósitos de turbiditos distais ou proximais e que fora depositada em irregularidades topográficas.

Souza et al. (1993) reconhecem que o empilhamento e a repetição dessas unidades informais sugerem que há uma natureza cíclica no registro, marcada por quatro tendências de sedimentação alternadas, denominados por ele de "Ciclos", dois regressivos e dois transgressivos (Figura 11). O Quadro 1 apresenta uma síntese com unidades litoestratigráficas propostas para Formação Salitre. 


\begin{tabular}{|c|c|c|}
\hline & CICLOS & Unidades informais \\
\hline \multirow{3}{*}{ IV } & \multirow{3}{*}{ TRANSGRESSIVO } & $z$ \\
\hline & & $\begin{array}{l}\text { JUSSARA } \\
\text { Superior }\end{array}$ \\
\hline & & JUSSARA Inferior \\
\hline \multirow{3}{*}{ III } & \multirow{3}{*}{ REGRESSIVO } & $\sum_{Z} Z$ SARANDI \\
\hline & & \\
\hline & & NOVA AMÉRICA ${ }_{\text {Inferior }}$ \\
\hline \multirow{2}{*}{ II } & \multirow{2}{*}{ TRANSGRESSIVO } & 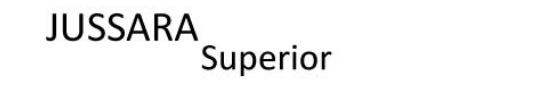 \\
\hline & & $\longrightarrow$ \\
\hline & \multirow{4}{*}{ REGRESSIVO } & $z<z^{\operatorname{IRECE}}$ \\
\hline & & NOVA AMERICA Superior \\
\hline & & $22 z 22$ \\
\hline & & $\begin{array}{l}\text { NOVA AMERICA } \\
\text { Inferior }\end{array}$ \\
\hline
\end{tabular}

Figura 11. Ciclos identificados na Formação Salitre, Bacia de Irecê, sinclinal de Irecê (SOUZA et al., 1993). A seção estudada está inserida nas subunidades Nova América Inferior e Nova América Superior, Ciclo Regressivo I, destaque. 
Quadro 1. Síntese com as unidades litoestratigráficas propostas para Formação Salitre.

\begin{tabular}{|c|c|c|c|c|c|c|c|c|c|c|c|c|c|c|c|}
\hline $\begin{array}{l}\text { Branner } \\
\text { (1911) }\end{array}$ & \multicolumn{2}{|c|}{$\begin{array}{l}\text { Oliveira e } \\
\text { Leonardos } \\
(1943)\end{array}$} & \multicolumn{2}{|c|}{$\begin{array}{l}\text { Brito- } \\
\text { Neves } \\
(1967)\end{array}$} & \multicolumn{2}{|c|}{$\begin{array}{l}\text { Pedreira } \\
\text { et al. } \\
(1975)\end{array}$} & \multicolumn{3}{|c|}{$\begin{array}{l}\text { Misi e Souto (1975) com } \\
\text { modificações de Misi } \\
\text { (1979) }\end{array}$} & \multicolumn{3}{|c|}{ Bomfim et al. (1985) } & \multicolumn{3}{|c|}{ Souza et al. (1993) } \\
\hline Série & Série & Fm. & Grupo & Fm. & Grupo & Fm. & Grupo & Fm. & Unidades & Fm. & Unidade & ubunidades & $\mathrm{Fm}$ & \multicolumn{2}{|c|}{$\begin{array}{l}\text { Unidades } \\
\text { Subunidades }\end{array}$} \\
\hline \multirow{5}{*}{ 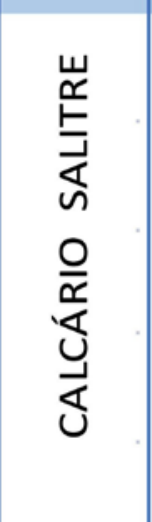 } & \multirow{5}{*}{ 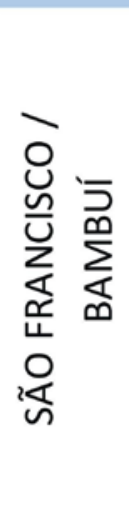 } & \multirow{5}{*}{ 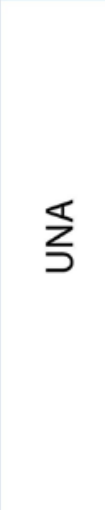 } & \multirow{5}{*}{$\sum_{\substack{\infty \\
\infty}}^{\infty}$} & \multirow{5}{*}{ 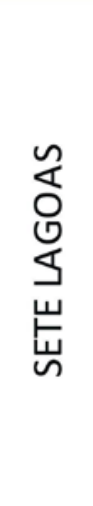 } & \multirow{5}{*}{ § } & \multirow{5}{*}{ 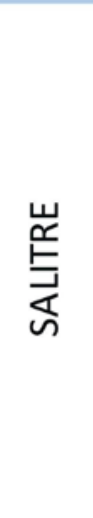 } & \multirow{5}{*}{ 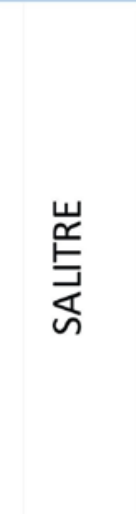 } & \multirow{5}{*}{ 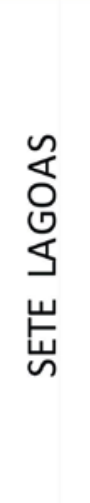 } & A1 & \multirow{5}{*}{ 崖 } & Jussara & $\begin{array}{l}\text { Superior } \\
\text { Médio } \\
\text { Inferior }\end{array}$ & \multirow{5}{*}{ 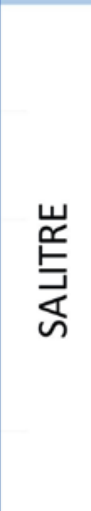 } & Jussara & $\begin{array}{c}\text { Superior } \\
\text { Inferior }\end{array}$ \\
\hline & & & & & & & & & A & & \multicolumn{2}{|c|}{ Irecê } & & \multicolumn{2}{|c|}{ Irecê } \\
\hline & & & & & & & & & B1 & & \multicolumn{2}{|c|}{$\begin{array}{c}\text { Nova América e } \\
\text { Gabriel }\end{array}$} & & \multicolumn{2}{|c|}{$\begin{array}{l}\text { Nova América } \\
\text { Superior** }\end{array}$} \\
\hline & & & & & & & & & B & & \multicolumn{2}{|c|}{ Sarandi } & & \multirow{2}{*}{\multicolumn{2}{|c|}{$\begin{array}{l}\text { Nova América } \\
\text { Sarandi } \\
\text { Nova América } \\
\text { Inferior }\end{array}$}} \\
\hline & & & & & & & & & $C$ & & \multicolumn{2}{|l|}{-} & & & \\
\hline
\end{tabular}




\section{CRITERIA TO HIGH-RESOLUTION STRATIGRAPHY OF A}

MICROBIAL-DOMINATED CARBONATE RAMP SYSTEM, NEOPROTEROZOIC SALITRE FORMATION, IRECÊ BASIN, NORTHEASTERN BRAZIL

Ana Virgínia Alves de Santana ${ }^{\text {a, b }}$; Farid Chemale Júnior ${ }^{\text {a, c }}$

a Instituto de Geociências, Universidade de Brasília, Campus Universitário, Asa Norte, 70910-900, Brasilia, DF, Brazil; ana.santana.unb@gmail.com

b Petroleum Geology Research Group, Instituto de Geociências, Universidade Federal do Rio Grande do Sul, Campus do Vale, Av. Bento Gonçalves 9500, 91509-900, Porto Alegre, RS, Brazil.

Programa de Pós-graduação em Geologia, Universidade do Vale do Rio dos Sinos, Avenida Unisinos, 950, CEP 93022-000, São Leopoldo, RS, Brazil. 
1/03/2017 Acknowledgement of receipt of your submitted article faridchemale@gmail.com - Gmail

Sedimentary Geology <eesserver@eesmail.elsevier.com>

para faridcj, mim

Dear Dr. Chemale Junior,

Your submission entitled "Criteria to high-resolution stratigraphy of a microbial-dominated carbonate ramp system, Neoproterozoic Salitre Formation, Irecê Basin, northeastern Brazil" has been received by Sedimentary Geology.

Your paper will be considered as belonging to the category Research Paper. Please contact us if this is not correct. Please note that submission of an article is understood to imply that the article is original and is not being considered for publication elsewhere. Submission also implies that all authors have approved the paper for release and are in agreement with its content. You will be able to check on the progress of your paper by logging on to https://ees.elsevier.com/sedgeo/ as Author. Your manuscript will be given a reference number in due course.

Thank you for submitting your work to this journal. Kind regards,

Elsevier Editorial System

4 CRITERIA TO HIGH-RESOLUTION STRATIGRAPHYOF A MICROBIALDOMINATED CARBONATE RAMP SYSTEM PÁGINA 51 


\section{Abstract}

The Salitre Formation records a Neoproterozoic carbonate sequence overlying the São Francisco into the central region of Bahia, northeastern Brazil. This study provides a unprecedented high-resolution stratigraphic framework of basal carbonate ramp from the Irecê Basin. The vertical succession shows different facies associations: middle ramp with microbial influence (FA1); shallow inner to the proximal middle ramp (FA2); inner ramp microbial-dominated (FA3); subaerial ramp/sabkha (FA4), that shows a typical framework of shallow marine carbonate developed in a homoclinal carbonate ramp. Sequential analysis at high resolution distinguished elementary and small scale sequence within intervals with a predominance of the FA3 and FA4. Elementary sequences (A-type) show typical thicknesses of the few tens of centimeters. These sequences were delineated in the field mainly from the identification of orderly factors series, such a facies or subfacies that by vertical succession demonstrated a periodicity/variability of the microbial growth. Small-scale sequences (B-type) are constituted by elementary sequences. Their demarcation occurred from sedimentological criteria and aid of the gamma ray log. Medium-scale sequences were delineated from the facies associations changes. One incomplete large-scale sequence was inferred and shown tens of meters thick. This sequence suggests a more significant cyclical change of environmental conditions. The high-resolution stratigraphic analysis method in Neoproterozoic marine microbial carbonate systems provided criteria that contribute to the development of predictive models in Neoproterozoic microbial carbonates which can be applied in areas of mineral exploration/exploitation as well as at the petroleum industry, for a better understanding of the flow lines in reservoirs in microbialite domain, or even in other reservoirs where there is a clear cyclic control, establishing the generation of different facies/diagenetic features, linked from variation in the base level.

Keywords: Microbialite; Sequence stratigraphy; Carbonate platform; Neoproterozoic; Irecê Basin; São Francisco Craton 


\subsection{INTRODUCTION}

The stratigraphic record of marine and lacustrine limestone successions show that their vertical facies tend to repeat regularly (e.g., Tucker and Wright, 1999; Dalrymple, 2010). This can be verified in diverse geological settings and scales, and according to Catuneanu et al. (2009; 2011) builds stratigraphic sequences. This inexorable pattern is found in carbonates worldwide at any depositional age (e.g., Grotzinger, 1986; Argenio et al., 1997; Strasser et al., 1999; Bádenas et al., 2010; Bialik et al., 2012; Al-Awwad and Collins, 2013; and others). These repeatedly vertical facies are commonly built due to periodic variations of the sea level (or lake level) changes. It normally occurs in response to cyclicity of the parameters astronomically controlled ("Milankovich Cycles"), implying a eustatic control on sedimentation (e.g., Montañez and Read, 1992). However, some authors point to a dominant tectonic signal as the main controlling factor for the deposition of cyclic carbonate successions (e.g., Bosence et al., 2009).

Recognition of metric scale carbonate sequences (high-frequency cycles) is needed both to unravel depositional controls for hydrocarbon exploitation (e.g., Lucia, 2007) as well as for barite, phosphate, zinc, and other economic mineral commonly found within this successions (e.g., Pawellek and Aigner, 2004). On the other hand, the highly fragmentary record prevents the precise reconstruction of these high-frequency cycles. This is due to some factors including low accommodation rates, which lead to non-deposition or erosion, and reworking of these sequences and commonly leading to condensation in carbonate successions (Strasser, 2015). For these reasons, methods aimed to improve recognition and 
correlations of high-frequency cycles in carbonate successions have been strongly encouraged.

The Salitre Formation consists of Neoproterozoic aged carbonate sequences deposited in the Irecê Basin within São Francisco Craton (Fig. 12A, B). These carbonate successions contain important mineral occurrences and deposits, including phosphate, zinc, and barite (Misi and Kyle, 1994). Irecê basin was deposited in an intracratonic setting (sag basin) after the breakup of the Rodinia supercontinent (ca. $900 \mathrm{Ma}$ ) and was later deformed during the Neoproterozoic Brasiliano/Pan-African orogeny (Chemale et al., 1993; Alkmim et al., 1996). Salitre Formation is exposed in the Paramirim Aulacogen at the central portion of the São Francisco Craton, within the Irecê, Una-Utinga, Salitre and Ituaçu synclines (Fig. 12B). Some of these sectors are marked by intense deformation, whereas in the study area, the S-SE segment of the Irecê syncline (Fig. 12B), sequences are deformed mainly by open to gentle folds and present well preserved sedimentary structures. Previous studies have recognized shallowing-upward cycles (Misi and Souto, 1975; Misi, 1979) and others indicate the existence of hundreds of meters thick transgressive-regressive cycles within Salitre Formation carbonates (Bomfim et al., 1985; Souza et al., 1993), but they were carried out on a regional scale. There is no high-resolution stratigraphic analysis available for this unit.

In this work, a high-frequency stratigraphic analysis of a microbial- dominated carbonate ramp in the Salitre Formation is presented with the definition of a high-order stratigraphic hierarchy. The main goal is to better understand the stratigraphic evolution and define 
practical criteria to assign a sequence hierarchy. In addition, criteria can be used to understand the primary mineral deposits that occur in the basin and to offers outcrop analogs of microbial- dominated sequences in Precambrian and Phanerozoic basins deposited on similar tectonic settings. Studies were conducted at detailed scale (1:20 scale) and derived from field investigations at escarpments generated by a complex of sinkholes, named Lapa Doce-Torrinha Cave, in the Diamantina Plateau (Chapada Diamantina), northeastern Brazil. In each doline is possible to measure detailed facies profiles, similar to borehole logging. Lapa Doce-Torrinha Cave is located in the S-SE domain of the western flank of Irecê Syncline. Strata dip $10^{\circ}$ to ENE in the study area (mean dip; Fig. 12B) and deformation is restricted to fracturing. This study was conducted in an area of ca. $2.8 \mathrm{~km}$ wide and $90 \mathrm{~m}$ high, oriented in the NNW to SSE direction, corresponding approximately to the depositional strike direction. Exposed units correspond to part of Unit B of Salitre Formation (Fig. 13).

\subsection{Geological Setting}

\subsubsection{REgional GEOLOGY}

The Irecê Basin crops out in an area of more than 31,000 square $\mathrm{Km}$, in a significant area of Bahia State, Northeastern Brazil. Expositions occur in the Paramirim Aulacogen (sensu Cruz and Alkmim, 2006; Fig. 12A, B), in a well-preserved portion of the São Francisco Craton. The Irecê Basin is correlative to the Bambuí Group in the São Francisco Basin, which crops out in the São Francisco Craton western portions (e.g., Dardenne, 1978; Misi, 1979; Misi et al., 2007). The Irecê Basin is deposited both in non-conformity over the cratonic basement 
(older than 1.8 Ga; Gavião Block; Barbosa and Sabaté, 2004) and in angular unconformity over the Paleoproterozoic to Neoproterozoic sequences of the Espinhaço Supergroup (deposited from 1.8 to 0.93 Ga; e.g., Guadagnin et al., 2015).

Irecê Basin includes Neoproterozoic units of Una Group/São Francisco Supergroup, and come into sight in five synclines located in the easternmost part of the Paramirim Aulacogen (Fig. 12B). It is formed by Bebedouro and Salitre formations (Pedreira et al., 1975; Misi and Veizer, 1998). Bebedouro Formation consists of glacial-marine deposits (Karfunkel and Hoppe, 1988; Guimarães, 1996; Figueiredo et al., 2009), and Salitre Formation comprises marine carbonate deposits with subordinate siliciclastic rock (e.g., Misi and Veizer, 1998; and reference therein), and occurs after an unconformity surface over glacial deposits and it is overlain by continental carbonate of Tertiary-Quaternary deposits from the Caatinga Formation (Branner, 1911). 


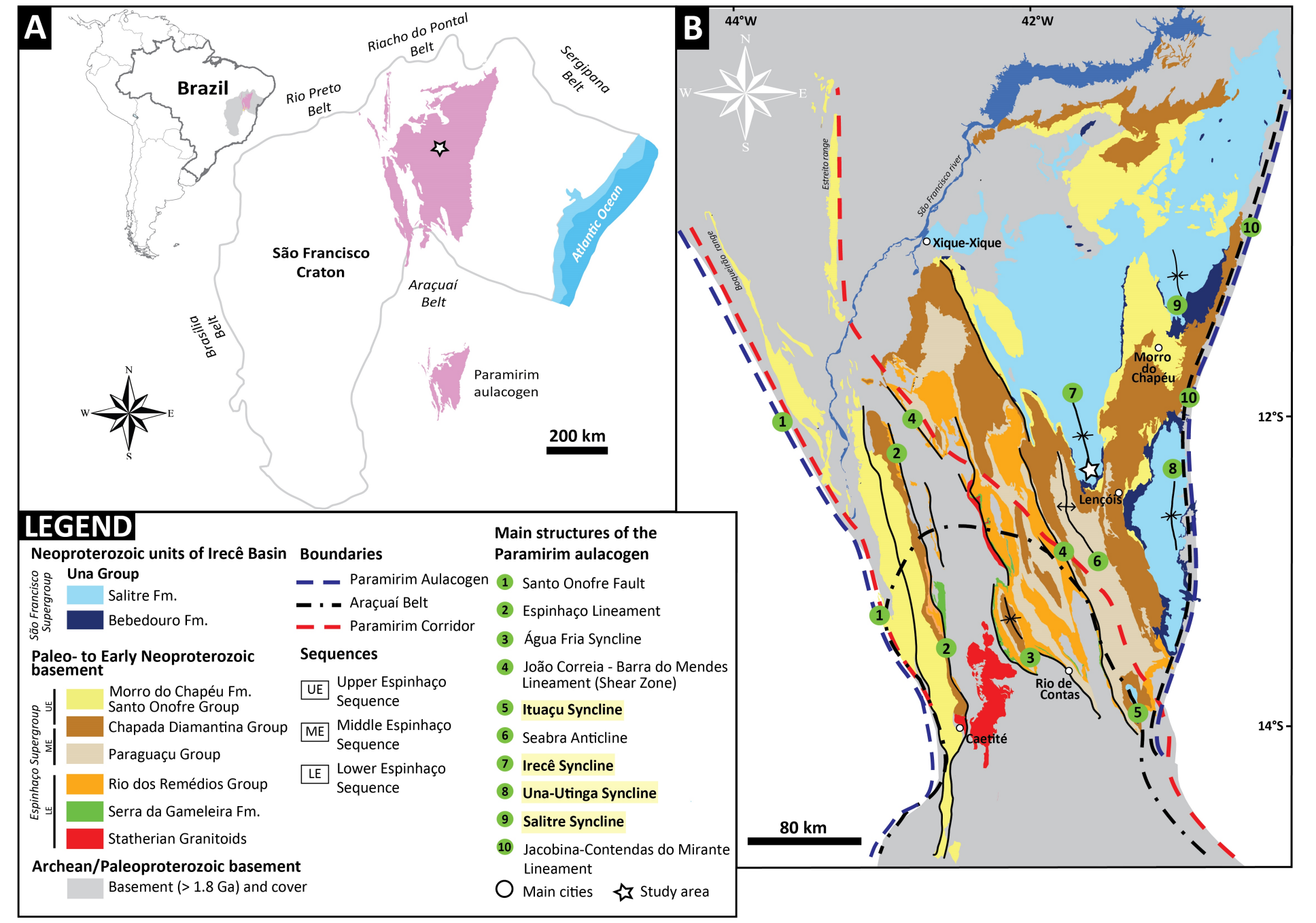

Figura 12. Geological context. (A) Sketch map showing the location of the study area in the scenario of São Francisco Craton. (B) Simplified geological map of the Paramirim Aulacogen and their main structures. The synclines in which the Salitre Formation crops out are highlighted in the legend by a yellow stripe (modified from Souza et al., 2003; Guimarães et al., 2012). 
The majority of published works for the basin assumes that Salitre Formation contains five informal units. Bottom to the top they are the C, B, B1, A and A1 units (Fig. 2; Misi, 1979; Misi and Kyle, 1994, Misi and Veizer, 1998; Misi et al., 2007; 2011). Unit C is formed by red argillaceous dolomite and stromatolites deposited in a restricted basin setting. This unit has been associated with cap dolomites, due to $\delta{ }^{13} \mathrm{C}$ values close to $-6.04 \%$ and aragonite crystal fans (Torquato and Misi, 1977; Misi and Kyle, 1994; Figueiredo, 2008). Unit B contains deep ramp and subtidal- to intertidal deposits (Misi and Kyle, 1994; Santana, 2016) and unit B1 is formed predominantly by supratidal deposits (Misi and Kyle, 1994). The vertical succession of Units B and B1 represent one typical shallowing-upward carbonate cycle (hundreds of meters thick), called Cycle I (Fig. 2; Misi, 1979). Units A and A1 comprise respectively deep platform and subtidal deposits. These units are interpreted as a second shallowing-upward carbonate cycle (Cycle II; Fig. 2; Misi, 1979). Units C, B and B1 are partially or totally equivalent to the Nova América, while the others, A and A1, are equivalent to the Irecê and Jussara units, respectively (Bomfim et al., 1985; Souza et al., 1993). 


\section{Stratigraphic column - Irecê Basin}

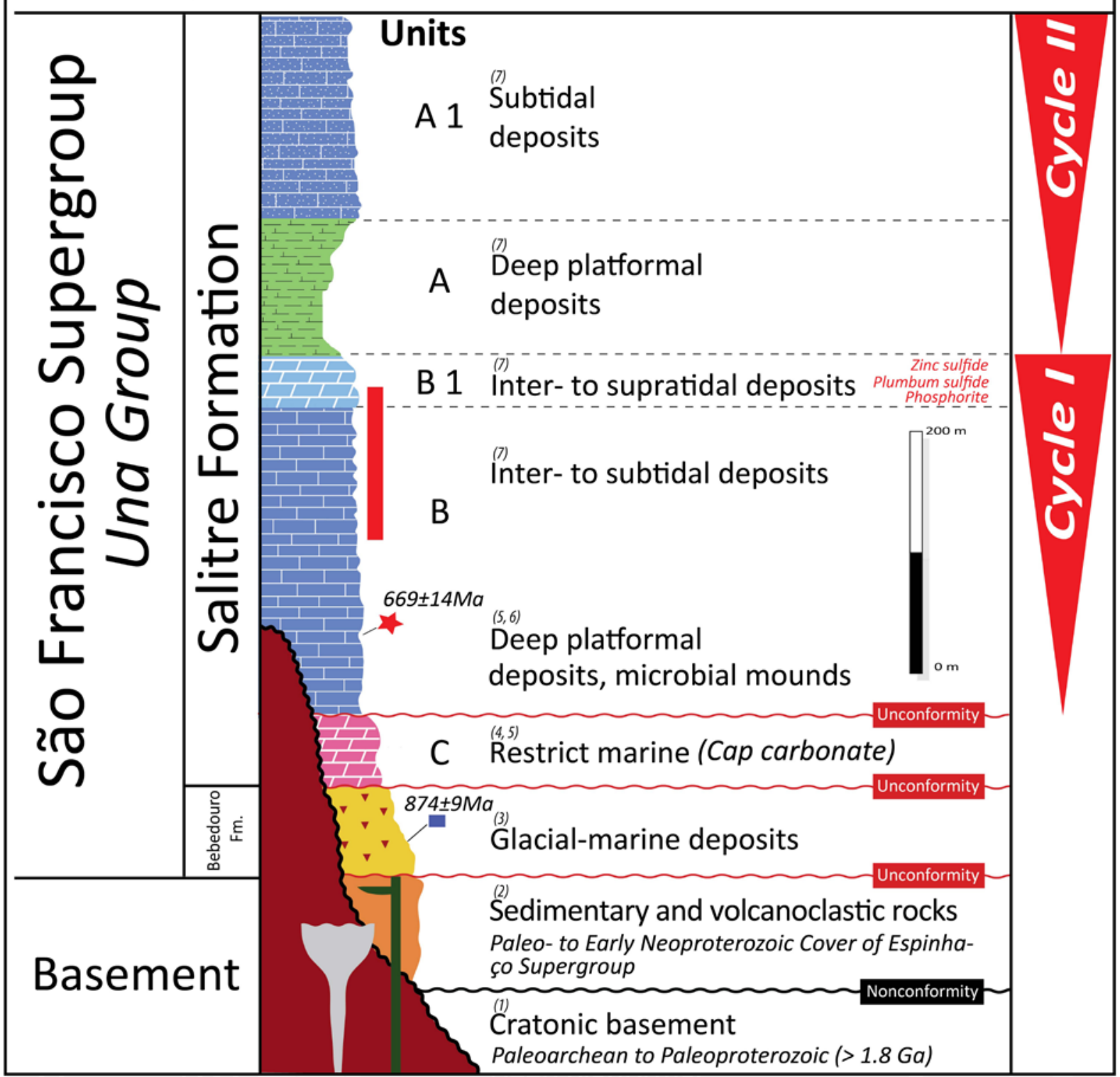

Figura 13. Stratigraphic column of the São Francisco Supergroup in Irecê Basin. The Salitre Formation was subdivided into five units and organized into two shallowing-upward regional cycles (hundreds of meters thick; Misi, 1979; Misi and Kyle, 1994). The red bar indicates localization of the section studied. Others references: (1) Barbosa and Sabaté, 2004; (2) Guadagnin et al., 2015; (3) Guimarães, 1996; (4) Torquato and Misi, 1977; (5) Figueiredo (2008); (6) Santana et al., in press; (7) Misi and Veizer, 1998. Square indicates maximum depositional age for Bebedouro Formation (Figueiredo et al., 2009), and star indicates maximum depositional age for Salitre Formation (Santana, 2016). 


\subsubsection{EVOLUTION AND FILLING OF THE IRECÊ BASIN}

The Irecê Basin filling and evolution are strongly linked with the development of West Gondwana paleocontinent. This depositional locus was generated by an initial fragmentation process associated with the break-up of the supercontinent Rodinia (ca. $900 \mathrm{Ma}$; Tonian rift stage; Silveira et al., 2013; and others). At study area, this record is represented by dike swarms dated at $934 \pm 14 \mathrm{Ma}$ (Loureiro et al., 2008), which intruded the entire Espinhaço Supergroup, and does not cut the São Francisco Supergroup units.

The Neoproterozoic Brasiliano/Pan-African orogeny has generated important structural features. At least two large-scale superimposed non-coaxial deformation patterns are distinguished, (1) NNW-SSE trending double plunging open folds, reverse faults and thrusts with western vergence, and (2) E-W striking thrusts faults verging to the south and flexural to shear folds (Chemale et al., 1993; Alkmim et al., 1996). Deformation decreases towards the south so that the southern segment of the N-S trending Irecê syncline records preserved sedimentary strata (Fig. 12B). However, the framework in which the basin was implanted is poorly known. In Irecê Syncline, structural and geophysical data suggest that the Irecê Basin was located on a faulted and tilted substrate with thickest sedimentary packages in the NW region, which it is characterized by the negative Bouguer anomaly (Duarte et al., 2012). Paleocurrent measurements of the Unit B1 indicate source area were located to the northeast/east of the study area (Leão and Dominguez, 1992).

4 CRITERIA TO HIGH-RESOLUTION STRATIGRAPHYOF A MICROBIAL- 
Sedimentological and provenance studies from glacial-marine deposits of Bebedouro Formation indicate a paleo-landscape feature controlled sedimentation (Guimarães, 1996; Figueiredo et al., 2009), which was possibly inherited from previous intracratonic extensional stages. Youngest detrital zircon grains dated at $874 \pm 9$ Ma sets the maximum depositional age for the Bebedouro Formation (Figueiredo et al., 2009). Misi et al. (2011), and reference therein, linked this unit to a Sturtian glacial event ( 717-660 Ma; Rooney et al., 2015), however, Caxito et al. (2012) based on ${ }^{87} \mathrm{Sr} /{ }^{86} \mathrm{Sr}$ signatures and other feature pointed that Bebedouro Formation was deposited during the end of the Cryogenian period, or in the Marinoan glacial event (ca. $635 \mathrm{Ma}$; Dunn et al., 1971; Halverson and Zhou, 2011). According to Santana (2016), basal portion of the Salitre Formation corresponds to sag setting such a gulf-like connected to a rift-related passive margin. The last depositional record in the Salitre Formation has been suggested to relate to a distal foreland context formed during the late to the post-orogenic stage of the Brasiliano Cycle. However, it is unclear due to lack of geochronological data and/or other evidence. The maximum depositional age of the Salitre Fm. has been constrained by the age of the volcanic zircon grain at $669 \pm 14 \mathrm{Ma}$, obtained from the tuffite at a lower Unit B (Fig. 13; Santana et al., in prep.).

\subsection{Methods}

Sedimentary logs were measured in outcrops along escarpments, at a 1:20 scale. Textures and main constituents were defined with aid petrographic analysis of the thin sections; however, parameters obtained from the fieldwork were the best used. Facies were described and

4 CRITERIA TO HIGH-RESOLUTION STRATIGRAPHYOF A MICROBIAL- 
interpreted on the basis of texture, variations in microbialite morphology, sedimentary structures, geometry, strata dimensions, and visual estimation of porosity type, as recorded in Dunham (1962), Logan et al. (1964), Embry and Klovan (1971), Choquette and Pray (1980), Demmico and Hardie (1994), Flügel (2010), Terra et al. (2010), and others. Different vertical facies successions allowed the definition of facies associations, or sub-environments, in accordance on the established in Dalrymple (2010). This enabled us to outline of a paleoenvironment setting.

Spectral gamma-ray logging of outcrops was done with a handheld gamma-ray spectrometer RS-230 BGO Super-SPEC model which provides samples concentration analysis with direct data display of $\mathrm{K}(\%), \mathrm{U}$ and Th (ppm). The samples were obtained from the bottom to the top with $20 \mathrm{~cm}$ regular space between them and orthogonal to the bedding, with one- minute counting times. Vertical techniques such as climbing and rappel were often needed to access to exposures in the escarpments. Total count of vertical gamma ray profile was plotted together with description facies and used qualitatively to indicate cycle stacking trends.

For sequence analysis, we assumed that a sequence corresponds to the geological record formed during a time interval when there is a change in accommodation or sediment supply. This concept enables the application of sequence stratigraphic studies at any spatial or temporal scale (Catuneanu et al. 2009; 2011). The method proposed in Strasser et al. (1999) enabled a hierarchy of the different documented sequences. Their rule is based on the recognition of 
diagnostic surface, or intervals, and their relative importance in the sedimentary record. The result of hierarchizing allowed correlation of the key surfaces and intervals of sequences of different thickness, i.e., different hierarchies. Transgressive-regressive sequence stratigraphic model (T-R sequence; Johnson and Murphy, 1984; Embry and Johannessen, 1992) was provided the conceptual approach and general nomenclature used. ANASETE ${ }^{\text {TM }}$ (Trademark of PETROBRAS) and PETREL ${ }^{\text {TM }}$ (Trademark of Schlumberger) software were used to analyze, interpret, and sharing of information. Lastly, photomosaic was used to illustrate the twodimensional (2D) sequences geometries.

\subsection{FACIES AND PALEOENVIRONMENTAL SETtIING}

\subsubsection{FACIES}

A total of $300 \mathrm{~m}$ of rock successions is described from 9 vertical sections. Ten facies and 8 subfacies have been described and interpreted (Table 1; Figs. 14, 15). They were categorized into three different groups, according to the depositional or post-depositional process (Dunham, 1962; Embry and Klovan, 1971; Flügel, 2010). Autochthonous carbonate facies represent the Group 1. They facies are formed in situ due to the interaction between benthic microbial communities and sediments (Microbialites; Burne and Moore, 1987). Therefore, the deposition reflects the direct or indirect action of microorganisms through metabolic processes in specific physicochemical conditions (Riding, 2000; Noffke et al., 2001, 2003; Dupraz et al., 2009). The morphology of the organosedimentary constructions and the proportions of automicrite were

4 CRITERIA TO HIGH-RESOLUTION STRATIGRAPHYOF A MICROBIAL- 
the criteria used to differentiate subfacies. Group 2 (Modified facies) congregates the facies formed by diagenetic process. Features indicating subaerial exposure are particularly diagnostic of the group (Tucker and Wright, 1990; Flügel, 2010). Allochthonous carbonate facies characterize the Group 3. The facies of this group have been deposited by hydrodynamics or gravitational processes. Microbial mats may be present.

\subsubsection{FACIES ASSOCIATION}

The windows study contains four depositional environments/facies associations, hereafter referred to as FA, distributed as the more distal position to the more landward setting as follows: (FA1) Middle ramp with microbial influence; (FA2) Shallow inner to proximal middle ramp; (FA3) Inner ramp microbial-dominated; and (FA4) Subaerial ramp/Sabkha. FAs are given briefly below.

4 CRITERIA TO HIGH-RESOLUTION STRATIGRAPHYOF A MICROBIALDOMINATED CARBONATE RAMP SYSTEMI PÁGINA 64 
Table 1. Summary of the main characteristic features of the facies types identified in the Salitre Formation in this study area (Article 1)

\begin{tabular}{|c|c|c|c|c|c|c|c|}
\hline Facies (Code) & $\begin{array}{l}\begin{array}{l}\text { Sub-facies } \\
\text { (Code) }\end{array} \\
\end{array}$ & Colour & Description & Geometry and extent & Depositional processes & Interpretation and occurrences & References \\
\hline \multicolumn{8}{|c|}{$\begin{array}{l}\text { AUTOCHTHONOUS CARBONATE FACIES GROUP } \\
\end{array}$} \\
\hline \multirow{4}{*}{$\begin{array}{l}\text { Stratiform } \\
\text { stromatolite (F1) }\end{array}$} & $\begin{array}{l}\text { Finely laminated } \\
\text { (F1a) }\end{array}$ & $\begin{array}{l}\text { Gray to light } \\
\text { gray in color, } \\
\text { and light brown } \\
\text { to ochre in } \\
\text { lamination color }\end{array}$ & $\begin{array}{l}\text { Fine-grained limestone; sub millimeter to millimeter thick, } \\
\text { alternating with irregular or crinkly laminations; often } \\
\text { discontinuous and broken lamina; hardgrounds; fenestral } \\
\text { porosity; pseudomorphs after evaporitic minerals; sometimes, } \\
\text { dolomitized; lamina with little or no synoptic relief; centimeter } \\
\text { to decimeter scale bed thickness. }\end{array}$ & $\begin{array}{l}\text { Stratiform and tabular forms } \\
\text { (biostromes). Extends to } \\
\text { hundreds of meters. }\end{array}$ & $\begin{array}{l}\text { Grain trapping and binding and direct } \\
\text { precipitation of automicrite in interaction with benthic microbial } \\
\text { communities; eventual subaerial exposure. }\end{array}$ & $\begin{array}{l}\text { Low energy settings; intertidal to } \\
\text { supratidal zones; inner ramp. }\end{array}$ & $\begin{array}{l}\text { Awramik and Semikhatov } \\
\text { (1979); Demmico and } \\
\text { Hardie (1994); Riding } \\
\text { (2000) }\end{array}$ \\
\hline & Laminated (F1b) & As above & $\begin{array}{l}\text { Fine-grained limestone, alternating massive until } 1 \mathrm{~cm} \text { thick } \\
\text { and irregular or crinkly continuous laminations; fenestral } \\
\text { porosity and pseudomorphs of evaporite minerals may occur; } \\
\text { sometimes, dolomitized; planar lamination prevails; centimeter } \\
\text { to decimeter scale bed thickness. }\end{array}$ & As above. & Trapping and binding, altermating with automicrite precipitation. & $\begin{array}{l}\text { Low energy settings; mainly intertidal } \\
\text { zones; inner ramp. }\end{array}$ & $\begin{array}{l}\text { 11 A wramik and Semikhatov } \\
\text { (1979); Demmico and } \\
\text { Hardie (1994); Riding } \\
\text { (2000) }\end{array}$ \\
\hline & $\begin{array}{l}\text { Coarsely } \\
\text { laminated (F1c) }\end{array}$ & $\begin{array}{l}\text { Massive: gray to } \\
\text { dark gray. } \\
\text { Laminae: dark to } \\
\text { brown }\end{array}$ & $\begin{array}{l}\text { Alternating massive layers (mudstone to wackstone) and } \\
\text { laminated intervals (flat to undulate/iriregular laminination); } \\
\text { massive layers: } 2 \text { to } 5 \text { centimeter thick; laminated layers: up to } \\
2 \text { centimeter thick; rare fenestral porosity associated with } \\
\text { lamina; increases in lamination convexity; autigenic euhedral } \\
\text { feldspar and gypsum crystals; centimeter to decimeter scale } \\
\text { bed thickness. }\end{array}$ & As above. & $\begin{array}{l}\text { As above, but with clear predominance of microbial action, or } \\
\text { physicochemical interactions, mediated } \\
\text { precipitation processes. }\end{array}$ & $\begin{array}{l}\text { Low energy settings; intertidal to } \\
\text { subtidal zones; supratidal pool; inner } \\
\text { ramp. }\end{array}$ & $\begin{array}{l}\text { Riding (2000); Flugel } \\
\text { (2010) }\end{array}$ \\
\hline & $\begin{array}{l}\text { Laminae convex } \\
\text { (F1d) }\end{array}$ & $\begin{array}{l}\text { Brown to ochre } \\
\text { in lamination } \\
\text { color }\end{array}$ & $\begin{array}{l}\text { Laminated with expressive convex form; laterally-linked } \\
\text { hemispheric (LLH); centimeter scale bed thickness. }\end{array}$ & As above. & $\begin{array}{l}\text { Trapping and binding dominated, and mineral precipitation by benthonic } \\
\text { microbiotas. }\end{array}$ & $\begin{array}{l}\text { Low energy settings; intertidal to } \\
\text { subtidal zones; inner ramp. }\end{array}$ & Logan et al. (1964) \\
\hline \multirow[b]{2}{*}{ Stromatolite (F2) } & $\begin{array}{l}\text { Small domes } \\
\text { (F2a) }\end{array}$ & $\begin{array}{l}\text { Brown to ochre } \\
\text { in lamination } \\
\text { color }\end{array}$ & Domal lamina; type-SH prevails; few centimeters thick beds. & Bioherms. & $\begin{array}{l}\text { Trapping and binding dominated, and mineral precipitation by benthonic } \\
\text { microbiotas. }\end{array}$ & $\begin{array}{l}\text { Low to moderate energy settings; } \\
\text { mainly subtidal zones; inner to } \\
\text { shallow inner ramp. }\end{array}$ & $\begin{array}{l}\text { Logan et al. (1964); Riding } \\
\text { (2000) }\end{array}$ \\
\hline & $\begin{array}{l}\text { Large columns } \\
\text { (F2b) }\end{array}$ & Gray & $\begin{array}{l}\text { Convex- to parabolic forms; finely spaced lamination, sub } \\
\text { millimeter to millimeter in thickness; laterally continuous (type- } \\
\text { LLH) or isolate bodies (type-SH); distinct loaf-shaped; up to } \\
50 \text { cm thick. }\end{array}$ & Biostromes and bioherms. & Trapping and binding dominated. & $\begin{array}{l}\text { Low to moderate energy settings; } \\
\text { mainly subtidal zones; inner to } \\
\text { shallow inner ramp. }\end{array}$ & Ridding (2011) \\
\hline
\end{tabular}

4 CRITERIA TO HIGH-RESOLUTION STRATIGRAPHYOF A MICROBIAL-DOMINATED CARBONATE RAMP 


\begin{tabular}{|c|c|c|c|c|c|c|c|}
\hline Facies (Code) & $\begin{array}{l}\begin{array}{l}\text { Sub-facies } \\
\text { (Code) }\end{array} \\
\end{array}$ & Colour & Description & Geometry and extent & Depositional processes & Interpretation and occurrences & References \\
\hline \multicolumn{8}{|c|}{ MODIFIED FACIES GROUP } \\
\hline \multirow{2}{*}{$\begin{array}{l}\text { Diagenetic } \\
\text { breccias (F3) }\end{array}$} & $\begin{array}{l}\text { Pseudobreccia } \\
\text { with tepees } \\
\text { (F3a) }\end{array}$ & Gray & $\begin{array}{l}\text { Breccia-like; "intraclastics" with mud to grain packstone; } \\
\text { partly dolomitized; tepees and desiccation cracks; } \\
\text { pseudomorphs after evaponitic minerals; f fenestral voids; } \\
\text { microbial mats; decimeter scale bedding thickness. }\end{array}$ & $\begin{array}{l}\text { Tabular. Extends to hundreds of } \\
\text { meters. }\end{array}$ & $\begin{array}{l}\text { E Early diagenetic processes; subaerial exposure; syndepositional } \\
\text { precipititation of evaporitit minerals; lime mud derived from } \\
\text { physicocheminica precipitation and/or microbial action. Grains, evidenced } \\
\text { by grainstone to packstones layers, may occurs due to the storm influence. }\end{array}$ & $\begin{array}{l}\text { Low energy settings; supratidal to } \\
\text { intertidal environments; arid climate; } \\
\text { inner ramp. }\end{array}$ & $\begin{array}{l}\text { Supratidal breccia of } \\
\text { Demmico and Hardie } \\
\text { (1994); Flügel (2010) }\end{array}$ \\
\hline & $\begin{array}{l}\text { Silicified } \\
\text { pseudobreccia } \\
\text { (F3b) }\end{array}$ & Dark & $\begin{array}{l}\text { Silicified microbialites and superposed brecciation; tepees and } \\
\text { desiccation cracks; locally disrupted or unclear; decimeter } \\
\text { scale bedding thickness. }\end{array}$ & $\begin{array}{l}\text { Tabular (replacement into } \\
\text { biostrome type) or irregular. } \\
\text { Extends to hundreds of meters. }\end{array}$ & $\begin{array}{l}\text { Evaporitic process, such a syndepositional precipitation and/or } \\
\text { replacement by evaporitic minerals. It may relate to hidrotermal. }\end{array}$ & $\begin{array}{l}\text { Low to moderate settings; mainly } \\
\text { supratidal to proximal subtidal } \\
\text { environments; arid climate; inner } \\
\text { ramp. }\end{array}$ & $\begin{array}{l}\text { Solution-evaporite- } \\
\text { collapse breccias of } \\
\text { Flügel (2010) }\end{array}$ \\
\hline \multicolumn{8}{|c|}{ ALLOCHTHONOUS CARBONATE FACIES GROUP } \\
\hline Laminite (F4) & & Gray & $\begin{array}{l}\text { Fine-grained limestone; thin lamina ( } 1 \text { to } 2 \mathrm{~mm} \text { thick), regularly } \\
\text { spaced and usually ymooth; crinkly lamina also occur; } \\
\text { undulose to low-angle cross-lamina; contorted beds, roll-up } \\
\text { structures, without prefered orientation; microbial mats; } \\
\text { terrigenous grains, silt-to very fine sand in size; decimeter to } \\
\text { meter scale bed thickness. }\end{array}$ & $\begin{array}{l}\text { Tabular. Extends to hundreds of } \\
\text { meters. }\end{array}$ & f Stom-influenced deposits; trapping and binding also occurs. & $\begin{array}{l}\text { Low to moderate energy settings; } \\
\text { intermediate ramp. }\end{array}$ & $\begin{array}{l}\text { Dumas and Amott (2006); } \\
\text { Muniz and Bosence (2016) }\end{array}$ \\
\hline $\begin{array}{l}\text { Massive mudstone } \\
\text { to wackestone (F5) }\end{array}$ & & Dark gray & $\begin{array}{l}\text { Lime mudstone to peloidal wackestone; massive, apparently } \\
\text { structureless or rare faint lamination; typical fine grain size } \\
\text { from silt to very fine sand; clotted peloidal microfabrics may } \\
\text { occur; bed sets with up to } 20 \mathrm{~cm} \text { thick. }\end{array}$ & As above. & $\begin{array}{l}\text { Suspension settling of carbonate mud and/or automicrite production (e.g., } \\
\text { microbial mediated). }\end{array}$ & $\begin{array}{l}\text { Low energy settings. Subtidal } \\
\text { environment. }\end{array}$ & Flügel (2010) \\
\hline $\begin{array}{l}\text { Cross-laminated } \\
\text { wackestone (F6) }\end{array}$ & & $\begin{array}{l}\text { Gray to dark } \\
\text { gray }\end{array}$ & $\begin{array}{l}\text { Lime mudstone to wackestone; wave ripples; fine to coarse } \\
\text { grains (peloids, intraclasts and terrigenous). Beds may be } \\
\text { separated by thin marble layers. }\end{array}$ & As above. & & $\begin{array}{l}\text { Low to moderate energy settings. } \\
\text { Distal intermediate ramp. }\end{array}$ & $\begin{array}{l}\text { Demicco (1985); Palma et } \\
\text { al. (2009); Labaj and Pratt } \\
\text { (2016) }\end{array}$ \\
\hline $\begin{array}{l}\text { Massive peloidal- } \\
\text { ooidal } \\
\text { packstone/wackest } \\
\text { one (F7) }\end{array}$ & & $\begin{array}{l}\text { Gra y to light } \\
\text { gray }\end{array}$ & $\begin{array}{l}\text { Packestone to wackstone; massive; peloids, microbialite } \\
\text { intraclasts, ooids and rare oncoids; grain size varies from fine } \\
\text { to very coarse sand; partly pyritized and dolomitized; } \\
\text { bituminous material occur; centimeter to decimeter scale bed } \\
\text { thickness. }\end{array}$ & $\begin{array}{l}\text { Tabular. Extension of few tens } \\
\text { of meters. }\end{array}$ & Ressedimentation (textural inversion). & $\begin{array}{l}\text { Moderate energy settings. Foreshoal } \\
\text { context on a shallow inner to proximal } \\
\text { intermediate ramp. }\end{array}$ & Flügel (2010) \\
\hline $\begin{array}{c}\text { Massive } \\
\text { intraclastic } \\
\text { peloidal } \\
\text { grainstone/packst } \\
\text { one (F8) }\end{array}$ & & Light gray & $\begin{array}{l}\text { Grainstone to packstone; massive; load (flame) structure at } \\
\text { bed base; intraclasts and peloids, fine to medium sand grains; } \\
\text { phosphatic grains and rip-up clasts occur, up to } 40 \mathrm{~cm} \text { thick. }\end{array}$ & Tabular. & $\begin{array}{l}\text { Hyperpycnal flows developed in response to with post-storm surge; } \\
\text { primary bedding structures may be full obliterated by fluidization or } \\
\text { diagenetic process. }\end{array}$ & $\begin{array}{l}\text { Moderate to high energy settings. } \\
\text { Deposition below fair-weather wave } \\
\text { base and above storm wave base. } \\
\text { Proximal intermediate ramp. }\end{array}$ & Flügel (2010) \\
\hline $\begin{array}{l}\text { Intraclastic } \\
\text { floatstone/rudston } \\
\mathrm{e}(\mathrm{Fg})\end{array}$ & & Gray & $\begin{array}{l}\text { Floatstone to rudstone; random internal fabric (i.e. intact, } \\
\text { thrusted, edgewise and disorganized); grains can range from } \\
\text { gravel to boulder size; sharp to few erosional base; poorly } \\
\text { sorted; bed amalgamation pronounced; bed sets of up to } 4 \mathrm{~m} \\
\text { thick; limited lateral continuity, sometime only few meters. }\end{array}$ & $\begin{array}{l}\text { Lenticular, wedge-shaped. } \\
\text { Short extent. }\end{array}$ & Reworked of the early cement lime mud by storms. & $\begin{array}{l}\text { High energy due to storm surge } \\
\text { influence; windward settings. } \\
\text { Common in proximal intermediate } \\
\text { ramp, but also occurs in inner ramp. }\end{array}$ & $\begin{array}{l}\text { Lithoclast limestone of } \\
\text { Flügel (2010) }\end{array}$ \\
\hline $\begin{array}{l}\text { Edgewise } \\
\text { conglomerates } \\
\text { (F10) }\end{array}$ & & $\begin{array}{l}\text { Clasts and the } \\
\text { fine-grained } \\
\text { matrix are gray in } \\
\text { colour }\end{array}$ & $\begin{array}{l}\text { Clast supported; clasts are flat to undulose, sheet-like or } \\
\text { irregular in shape, and edgewise, imbricated or disorganized in } \\
\text { fabric; grains range from gravel to couble in size; locally } \\
\text { shows scoured bases; stylolite, moldic porosity and } \\
\text { pseudomorphs after evaporitic minerals are common; } \\
\text { dolomitization; few centimeters up to } 30 \mathrm{~cm} \text { thick. }\end{array}$ & $\begin{array}{l}\text { Lenticular, rarely tabular, with } \\
\text { stratiform stromatolites } \\
\text { delimiting bed base and top. } \\
\text { Short extent. }\end{array}$ & $\begin{array}{l}\text { Fragments of desiccated tidal flat locally reworked by wave and/or current } \\
\text { as "wash deposits". }\end{array}$ & $\begin{array}{l}\text { Moderate energy. Common in } \\
\text { intertidal zone. }\end{array}$ & $\begin{array}{l}\text { Type A flakestone of } \\
\text { Tucker (1977); Shin (1983) }\end{array}$ \\
\hline
\end{tabular}

4 CRITERIA TO HIGH-RESOLUTION STRATIGRAPHYOF A MICROBIAL-DOMINATED CARBONATE RAMP 

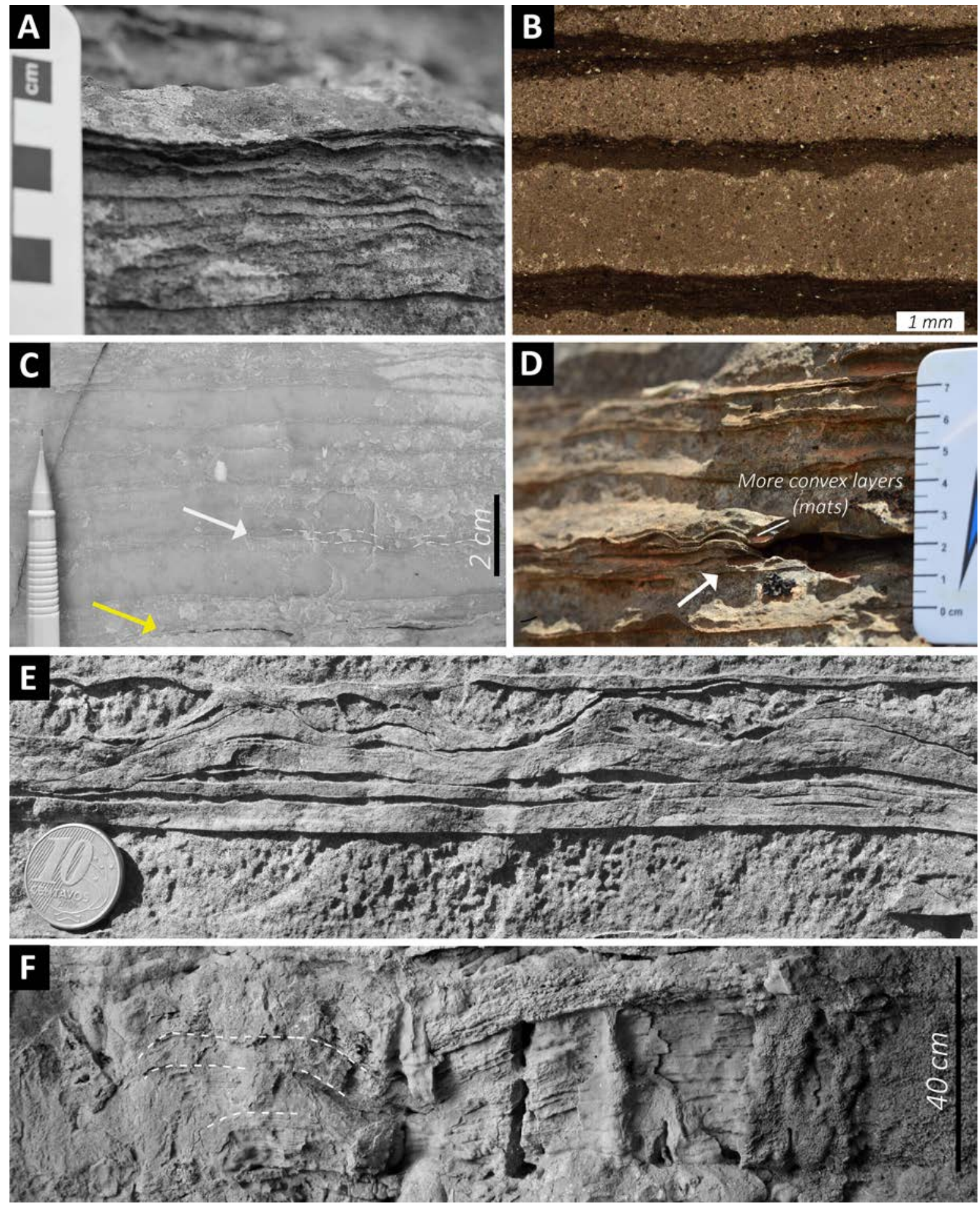

Figura 14. Facies recognized in the windows study. (A) Stratiform stromatolite, finely laminated (F1a subfacies); (B) Thin section of the F1a subfacies, $\mathrm{N} / /$; (C) Note an increase of the porosity within finely laminated subfacies (yellow arrow) and convex microbial mats within of the laminated subfacies (F1b subfacies; white arrow); (D) Coarsely laminated (F1c subfacies) with more convex microbial mats (white arrow); (E) Stratiform stromatolite whit laminae convex (F1d subfacies). Coin for scale is $2 \mathrm{~cm}$ in diameter; (F) Stromatolite with large columns (F2b subfacies). Highlights for the loaf-shaped-type (dashed).

4 CRITERIA TO HIGH-RESOLUTION STRATIGRAPHYOF A MICROBIALDOMINATED CARBONATE RAMP SYSTEMI PÁGINA 67 

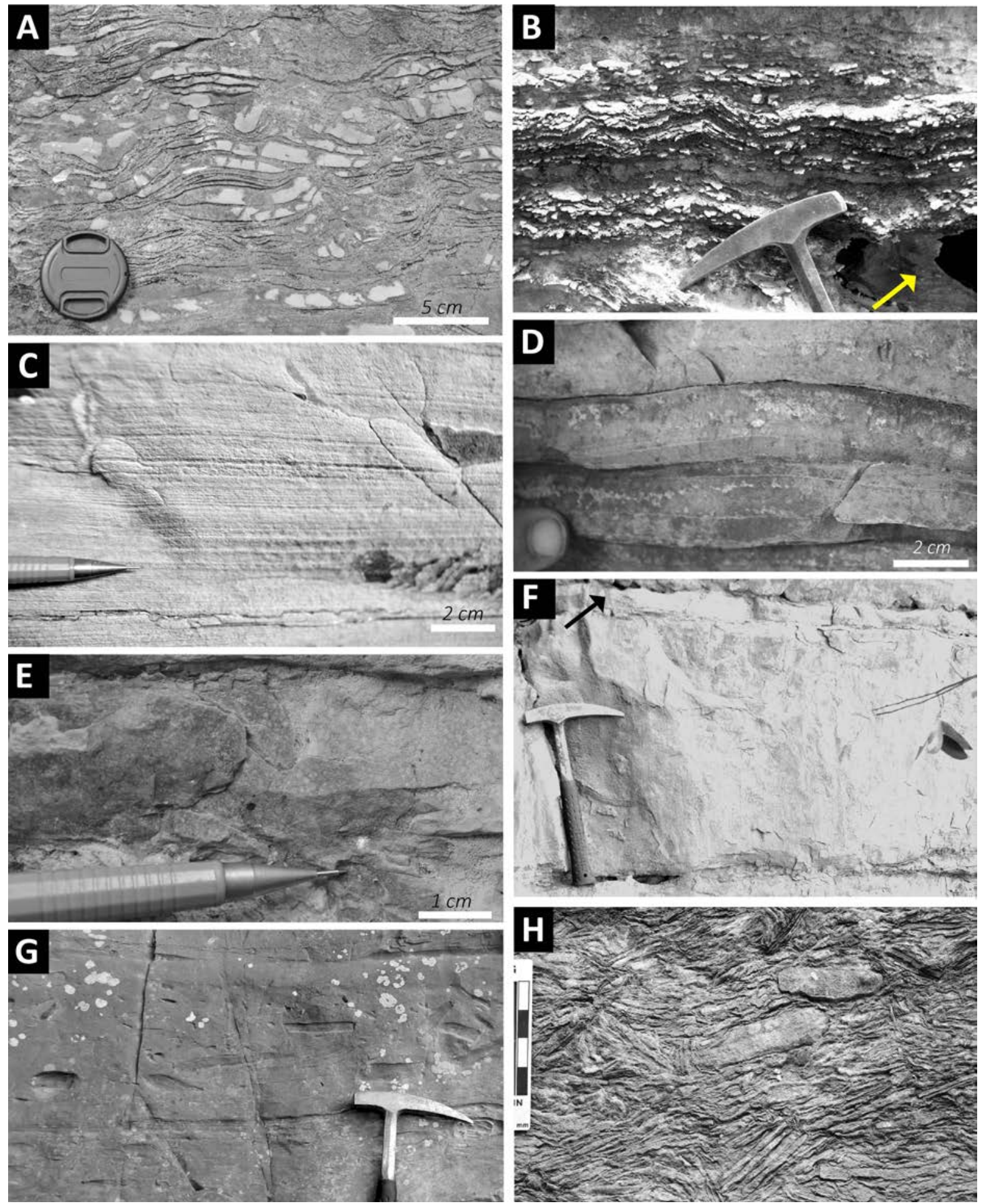

Figura 15. Facies recognized in the windows study (continuation). (A) Pseudobreccia with tepees (F3a subfacies); (B) Silicified pseudobreccia (F3a subfacies). Note an increase of the porosity (yellow arrow); (C) Laminite (F4 facies); (D) Cross-laminated wackestone (F6 facies); (E) Massive peloidal-ooidal packstone/ wackestone (F7 facies); (F) Massive intraclastic peloidal grainstone/packstone (F8 facies). Highlights for the load (flame) structure at bed base (black arrow); (F) and (H) Intraclastic floatstone/rudstone (F9 facies). 


\section{MIDDLE RAMP WITH MICROBIAL INFLUENCE (FA1)}

\section{$\underline{\text { Description }}$}

This facies association comprises laminite and cross-laminated wackestone (F4 and F6; Table 1). F4 facies consists of the lighter micropeloidal and darker microbial laminae that show mm- scale lamination with two types of laminae: crinkly or smooths surfaces (Fig. 15A). The thinly laminated is flat but also are described $\mathrm{cm}$ - scale roll-up structures and low angle cross-stratification. F6 facies consists of the cross-laminated wackestone, with fine- to coarse grains (peloids, intraclasts and terrigenous). Their beds may be separated by thin marly layers. FA1 exhibit geometry strictly tabular with lateral extension of the order of tens- to hundreds of meters.

\section{Interpretation}

Wave ripples and undulate to low angle cross stratification suggest the action of oscillatory process (Dumas and Arnott, 2006). The presence of the microbial mats (F4 facies) indicates microbial- mediated carbonates precipitation and grain trapping and binding process. Rollup structures can be evidence both an in situ microbial mat action and soft-sediment deformation structures (Simonson and Carney, 1999; Pruss et al., 2010). Wackestone texture (F6 facies) needs a tranquil depositional condition (Flugel, 2010). Thus, F4 and F6 facies require quiet water into low- energy environment alternating with moderate depositional energy. Similar facies has been interpreted as deposits of deep subaqueous setting (Grotzinger and Al-Rawahi, 2014; Muniz and Bosence, 2016; Labaj and Pratt, 2016). Here, FA1 is associated with the deepest setting of the study area, formed in a subaqueous environment, between fair-weather wave base and storm wave base. 


\section{SHALLOW INNER TO PROXIMAL MIDDLE RAMP (FA2)}

\section{$\underline{\text { Description }}$}

This facies association consist mostly of intraclastic floatstone/rudstone (F9 facies), with oolitic lime grainstone- packstone matrix, and intraclasts of microbialite. These beds show lenticular or wedge-shaped geometry, rarely tabular, and are amalgamated with sharp to few erosional bases. F9 is interleaved with massive peloidal-ooidal packstone/wackestone (F7 facies) and massive intraclastic peloidal grainstone/packstone (F8 facies). Despite having a wide area of occurrence, the thickness of the FA2 is quite variable, from tens of centimeters to several meters.

\section{Interpretation}

The facies indicate deposits generated by a re-sedimentation process, common on storminduced deposits (Demmico and Hardie, 1994). For example, ooids as the main allochemical grains of the mud- rich facies (F7) implies the occurrence of textural inversion, with grains typical of high-energy environments, such as shoals, transported to lower depositional energy configurations through re-sedimentation action. The occurrence of these facies suggests a high- to moderate energy depositional conditions, near or below fair-weather wave base and above storm wave base, probably linked on a shallow inner ramp, such a complex shoals, to a proximal middle ramp (Amour et al., 2012). 


\section{INNER RAMP MICROBIAL DOMINATED (FA3)}

\section{$\underline{\text { Description }}$}

FA3 is mainly characterized by facies, and their subfacies, which comprises of autochthonous carbonate facies group (Stratiform stromatolite (F1) and stromatolite (F2); Table 1), with variable thickness (centimeter- to decimeter-thick). Other not so common facies are edgewise conglomerates (F10), up to $30 \mathrm{~cm}$ thick, and intraclastic floatstone/rudstone (F9), until up $400 \mathrm{~cm}$ thick. FA3 forms packages with tabular geometry and expressive lateral tracing.

\section{Interpretation}

F1 and F2 and their subfacies indicate microbialite builds from grain trapping/binding and/or direct precipitation of automicrite in interaction with benthic microbial communities (e.g., Riding, 2000; Noffke et al., 2001, 2003; Dupraz et al., 2009). Their morphologies type allowed interpreting FA3 as an inner ramp, more precisely the proximal intertidal/subtidal domain (e.g., Logan et al., 1964). The presence of the F10 facies, which may be associated with the supratidal/proximal intertidal settings, strengthens this interpretation (Shinn, 1983). Restricted occurrence of the F9 indicates reworked of the early cement lime mud by stormssurge (Demmico and Hardie, 1994).

\section{SUBAERIAL RAMP/SABKHA (FA4)}

\section{$\underline{\text { Description }}$}

FA4 comprises diagenetic breccias (F3 facies; sensu Flugel, 2010), and stratiform stromatolites (F1), mainly their F1c subfacies. F3 is subdivided into 2 subfacies. 
Pseudobreccia with tepees (F3a) congregates "intraclastics" with mud- to grain-packstone textures and partly dolomitized. Microbial mats may be common. Silicified pseudobreccia (F3b) consists of silicified microbialites with superimposed brecciation. Both show pseudomorphs after evaporitic minerals, as also tepees and desiccation cracks structures, and thickness up to few tens centimeters. At massive levels of F1, gypsum (sulfate mineral) and authigenic euhedral feldspar may occur in the automicrite. FA4 is strictly tabular and is concentered in the top of the stratigraphic TOR 4 section.

\section{Interpretation}

Macroscopic and microscopic features of the facies indicate chemical and/or microbial induced precipitation of carbonate (automicrite) followed by subaerial exposure as well as actuation of diagenetic processes, such as early marine cementation (e.g., Tucker, 1976; Assereto and Kendal, 1977; El Tabakh et al., 1999). These features are diagnostic of deposition in an environment with low energy, such a saline to hypersaline settings, into within limits of the protected inner ramp, under arid climate. Silicified or replaced by calcite nodules are a common occurrence in carbonates formed in environments of proximal and supratidal- type sabkha and authigenic euhedral feldspar and gypsum crystals are good indicators of post-depositional deposition in supratidal/sabkha environments. Grains, evidenced by grainstone to packstone layers, may occur due to storm action. 


\subsection{SEQUENCE STRATIGRAPHY FRAMEWORK}

Sequential analysis at high resolution distinguished elementary and small-scale sequence only within intervals with a predominance of the FA3 and FA4.

\section{ELEMENTARY SEQUENCES}

A complete sedimentary cycle, that is, facies/subfacies or features indicative of a tendency to deepening followed by features that indicate shallowing (Fig. 16) circumscribes an elementary sequence, here so called sequence A, or sequence A-type. Into within limits of on a shallow ramp such a FA3 and FA4 facies associations, sequence A may be pointed from the sequencing-based mainly on the morphological variation of the stratiform stromatolites (F1 facies, and their subfacies; Fig. 16A), and on the presence of facies indicative of more shallow settings, such a edgewise conglomerates (F10 facies; Fig. 16B) and/or diagenetic features which suggests subaerial exposure (F3 facies; Fig. 16C). A maximum flood zone (mfz A-type) can be marked by the occurrence of microbialite subfacies whose growth requires a higher water depth (e.g., F1d subfacies, Figs.14D), or by the presence of facies such as massive mudstone to wackestone (F5 facies; Fig. 15D). For example, an ideal stratiform stromatolite succession of the elementary sequence A-type is formed from bottom to top by F1b subfacies, followed by F1c, F1d, F1c, F1b and F1a subfacies (Fig. 16A).

Sequence $A$ is easily recognizable in the field because its limits commonly mark discontinuities in outcrops (Fig. 17). In general, within the limits the same sequence A-type, total count of vertical gamma ray profile pointed to lower values associated with the 
shallowing facies, and relatively higher values, associated with the facies that suggest deeper sea water.

Dome and columnar stromatolites (F2a and F2b subfacies) not included in the cyclical analysis. These subfacies were described in the LDO-3, LDO-1, TOR-5 and TOR-4 sections, just in the passage from FA3 to FA2. The level at which they occur can be correlated laterally, mainly in the TOR-5 and TOR-4 sections. Sometimes on a top or nearly of the stromatolites bodies may be marked by erosion surface.

\section{SMALL-SCALE SEQUENCES}

A small-scale sequence, here named by "sequence B-Type", consists of successive sequences A-type. The criteria for their delimitation are (1) sedimentological data (Fig. 18) and (2) evaluation of the total count of vertical gamma ray profile. A first criterion is made from the analysis of the vertical arrangement of the different sequences $A$. For example, the tendency of shallowing in a sequence B is associated with the occurrence of facies indicative of less accommodation availability of a sequence $A$, where the thickness of this facies is anomalous, often with abundant structures that suggest subaerial exposure, as shown in Figure 18. The second criterion, however, requires the evaluation of the total count of vertical gamma ray log and specifies observations of the Th and $U$ radioelements given two steps. The first need identifies the highest total count values that have a clear correspondence to the interval identified in an elemental sequence $A$ as an increase of accommodation, indicates a maximum flood zone ( $\mathrm{mfz}$ B-type). The second is identifying between two mfz-B-type the facies with features indicates of subaerial exposure, such a 
pseudobreccia with tepees facies (F3a). Normally this facies will linked with lowest total count of vertical gamma ray log present between the two different $\mathrm{mfz}$ B-type and coincide with a maximum regressive surface of the sequences $A$. This surface will be too a maximum regressive surface of the sequences B. Sequences B is easily recognized from sedimentological parameters mainly of the intervals marked by the succession between facies of FA3 and FA4.

\section{Medium-sCALe SEQUenCE}

A total of six medium-scale sequences hereafter referred to as sequences C-type were recognized in the study window. From the bottom to the top are called C1, C2, and C3... respectively, and are vertically arranged into a few tens of meters thick. These sequences were delineated from the FA changes; implies in vertical succession between the different FA types, antagonistic into proximity or distality, in relation to the coastline.

The sequence $\mathrm{C} 1$ is very incomplete (ca. 1,7 m; section LDO-2). C2 is complete and shows at least $38 \mathrm{~m}$ thick. C3 is probably complete and exhibits approximately $40 \mathrm{~m}$ thick (Fig.19). The sequence $\mathrm{C} 1$ being with transgressive deposits represented mainly by stromatolites (F1 - subfacies F1a, b and $c$; and F2, subfacies F2a) of the FA 3. A maximum flooding zone, codified by "mfz C-type 1" is suggested due to a thicker FA1 interval, which exhibits most high values of the gamma ray logs. This zone closes the transgressive systems tract (TST; Strasser et al., 1999; Catuneanu et al. 2010; 2011). The sequence C2 shows another transgressive phase, mainly represented by FA3. Some silicified levels may be associated with a proximal intertidal domain. 
The occurrence of significant levels of intraclastic floatstone/rudstone (F9) may be associated with FA3 or FA2. It is unclear, and the lack of correlation with other sections precludes a better interpretation. At the time, it is linked to FA3, which passes vertically to an important interval of FA1 (LDO-2A and TOR-4 sections). Gamma ray logs exhibit high values and a maximum flood zone ( $\mathrm{mfz} \mathrm{C}$-type 2 ) was inferred at the base of the section LDO_2A. This zone is correlated with FA1 intervals at the base of the TOR_4 section. It is also presented an anomalous gamma value total.

The regressive systems tract (RST; Johnson and Murphy, 1984; Embry and Johannessen, 1992; Catuneanu et al. 2010; 2011) is characterized by FA3 domain and is best exposed in the LDO_2A and TOR_4 sections. A maximum flood zone (mfz C-type 3 ) is inferred, but unclear. Regressive systems tract (mrs C-type 3) is suggest from base of the LDO_6 and LDO_3 sections, there is little thickness, but these are the best profiles for field visualization of the maximum regressive surface (mrs C-type 3 ). This surface is equivalents on a sequence boundary of a medium scale sequence, and limits sequences C3 and C3. In the LDO_6 section this mrs C-type 3 is characterized by the concentration of features indicative of shallow waters, as F1a subfacies, fenestrae pores- rich. In the LDO_3 section, it is observed that the top layers of the C3 sequence present a pinch-out to the north. Spectral gamma data associated with the sequence boundary between the sequence $\mathrm{C} 3$ and $\mathrm{C} 4$ show a change in the general signature, especially when the total count channel is analyzed. 
(A)
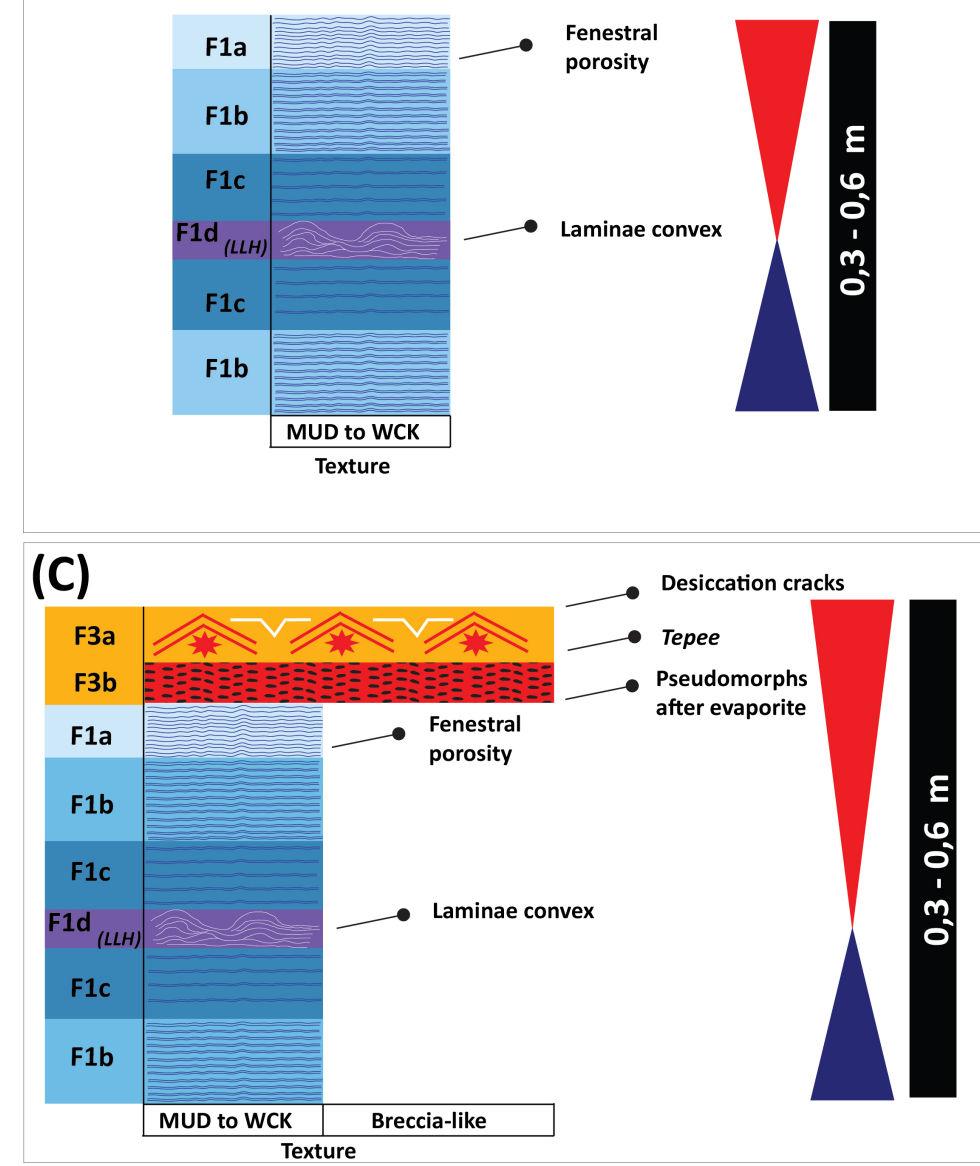

Key Accommodation Paleoenvironments

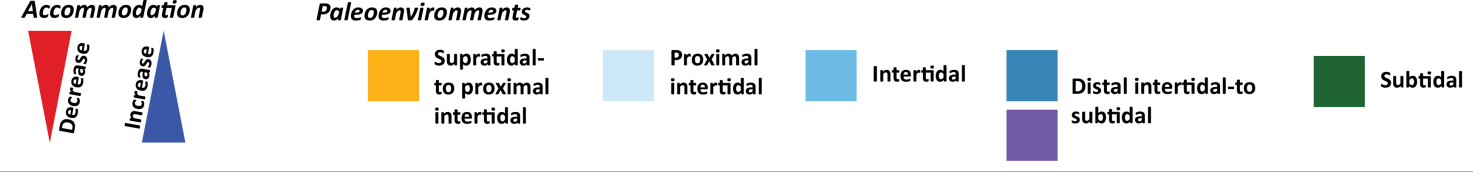

4 CRITERIA TO HIGH-RESOLUTION STRATIGRAPHYOF A MICROBIAL-DOMINATED CARBONATE RAMP SYSTEMI PÁGINA 77
Figura 16. Different elementary sequences recognized in the study area. 


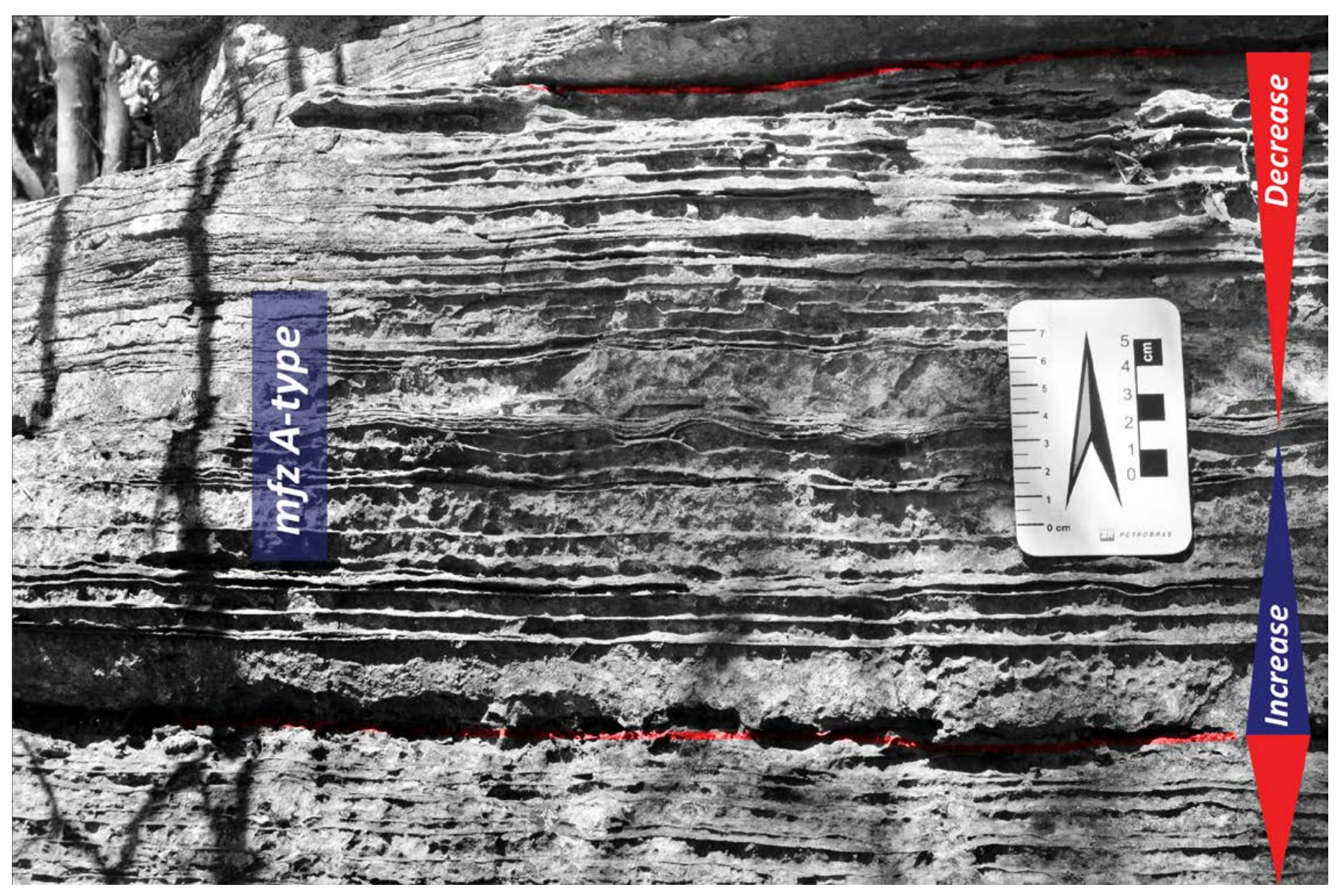

Figura 17. Visualization of a maximum flooding zone of the sequence elementary (mfz A-type). Sequence $A$ is easily recognizable in the field because its limits commonly mark discontinuities in outcrops.

4 CRITERIA TO HIGH-RESOLUTION STRATIGRAPHY OF A MICROBIAL-DOMINATED CARBONATE RAMP SYSTEMI PÁGINA 78 


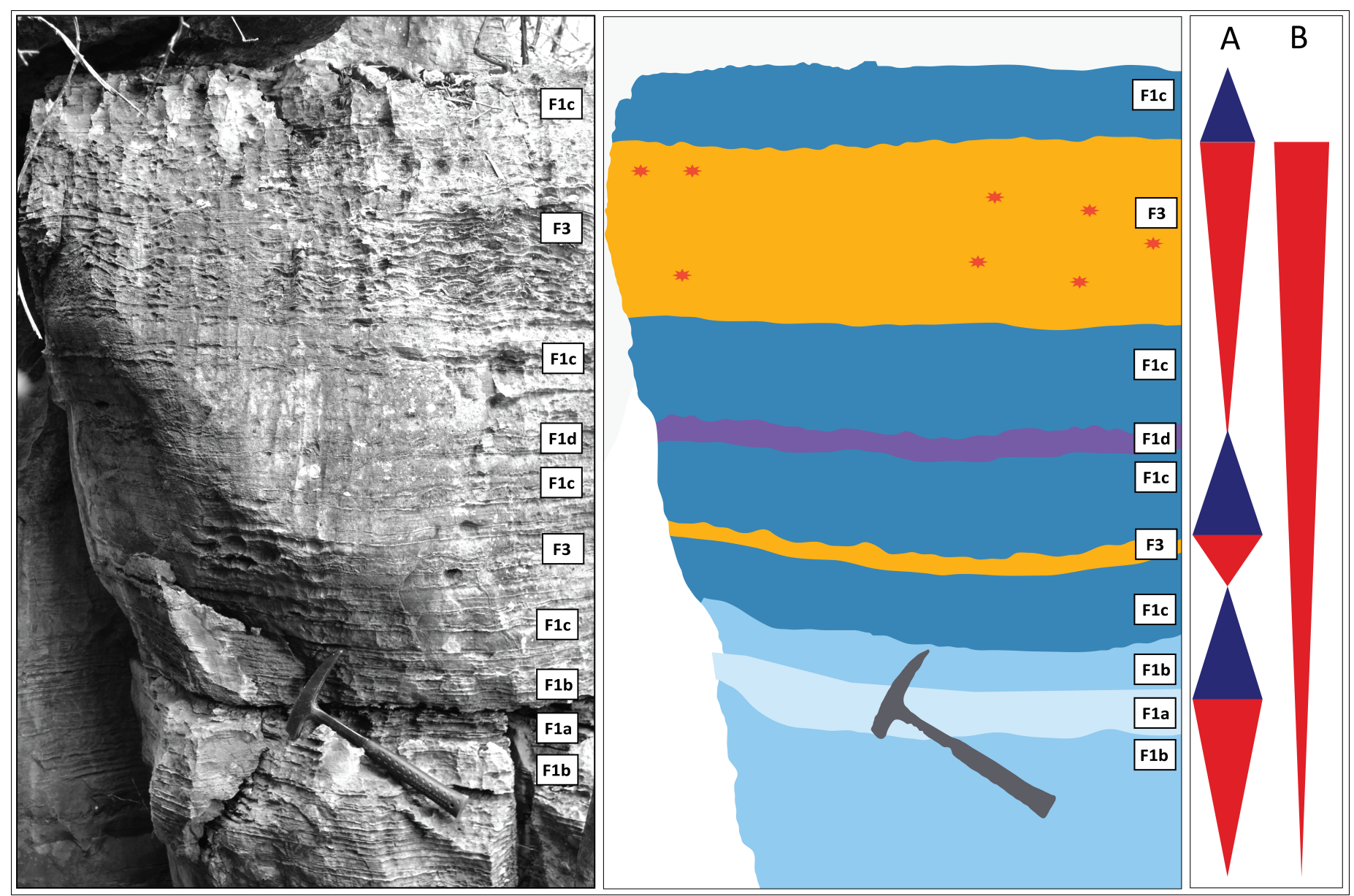

Figura 18. Vertical arrangement of the different sequences $A$ allows observed tendency of shallowing in a sequence $B$, normally associated with the occurrence of facies indicative of less accommodation availability of a sequence $A$, where the thickness of this facies is anomalous, often with abundant structures that suggest subaerial exposure. See Table 1 for facies and subfacies descriptions and Fig. 16 to legend. 
The sequence C4 begins with a predominance of facies correlated to FA3. Their transgressive systems tract ends with a thick portion with the F1c, LDO-6 section. A new regressive systems tract ends at the top of FA3 with deployment of the FA1 and / or FA2. Sometimes on a top or nearly of the stromatolites bodies may be marked by erosion surface (mrs C-type 4).

In the LDO 2 sections, there is an outstanding level of large columnar stromatolites, domal stromatolites before the implantation of these faciological associations. In the different sections, a maximum flood zone ( $\mathrm{mfz}$ C-type 5 ) was inferred from cited facies associations levels. Other regressive systems tract is marked by the succession between facies of FA3 and FA4, with a clear predominance of the FA4 to the top, especially of the diagenetic breccia (F3 facies; mrs C-type 5), following a clear regressive trend until that form a tepee belt intervals, highlights at top of the TOR-4 section (Fig. 19). Different system tract context of the sequence C-type shows different sequences A-type. Consequently, the content of a Btype sequence, and also its thickness, will vary in response according to the characteristics of the elementary sequences constructing it.

\section{LARGE-SCALE SEQUENCE}

One large-scale sequence is inferred and is incomplete. This sequence is named by "sequence D-type" and comprises the medium-scale sequences before described. Sequence D have tens of meters thick. Into their vertical organization occurs a maximum flooding zone, indicates from concentration of the $\mathrm{mfz}$ C-type, and a maximum regressive surface (mrs Dtype), that coincide with the mrs C-type 3 marked on a tepee belt (Fig.19).

4 CRITERIA TO HIGH-RESOLUTION STRATIGRAPHYOF A MICROBIALDOMINATED CARBONATE RAMP SYSTEMI PÁGINA 80 


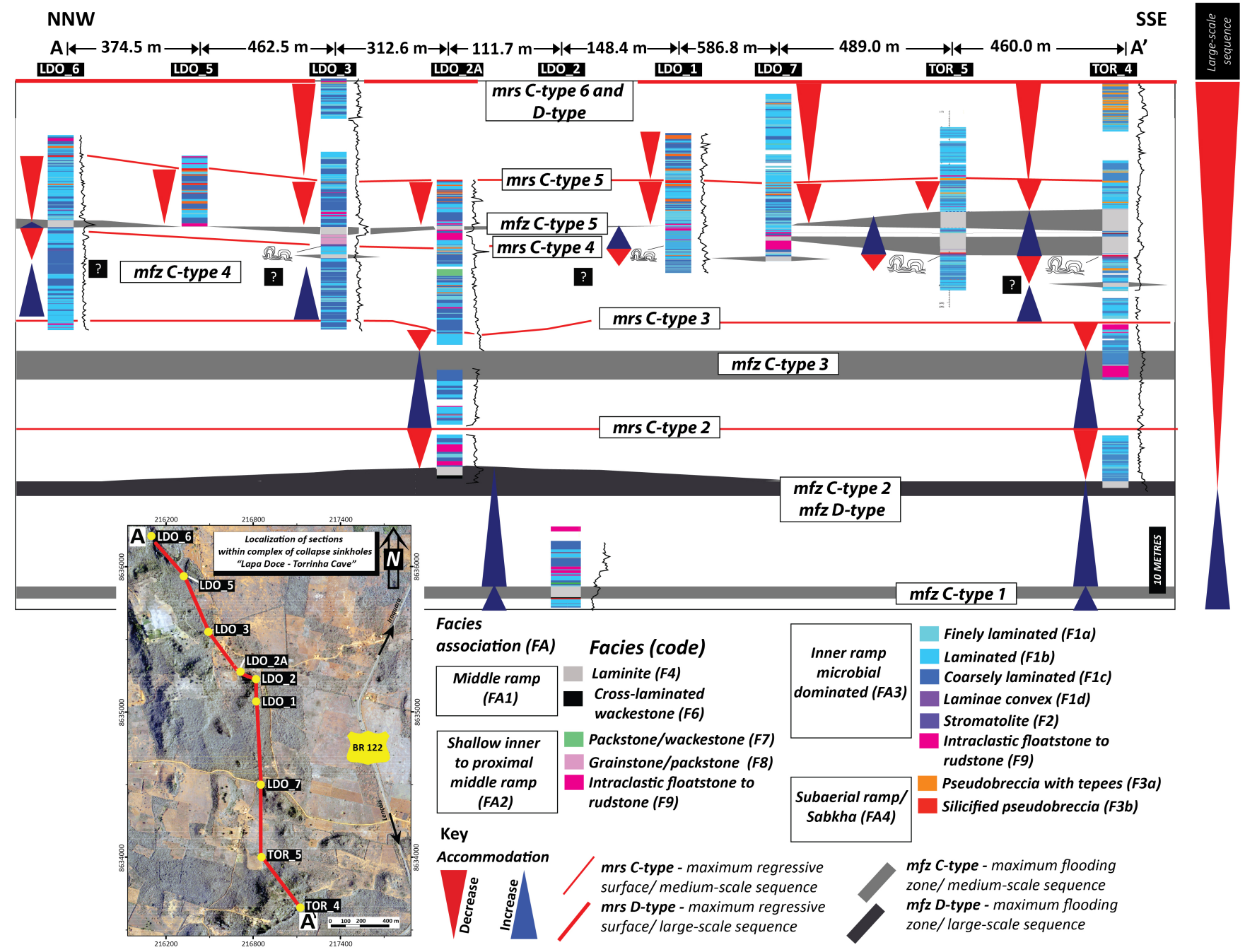

Figura 19. Cross-sections correlates the medium-scale sequences described.

4 CRITERIA TO HIGH-RESOLUTION STRATIGRAPHY OF A MICROBIAL-DOMINATED CARBONATE RAMP SYSTEM | PÁGINA 81 


\subsection{RAMP FEATURES}

Facies associations described corresponds to a typical framework of shallow marine carbonate system (Fig. 20; Burchette and Wright, 1992) which possibly developed in a platform whose form that best adequate is a homoclinal carbonate ramp, as referred by Read (1982), due to (1) absence facies deposited by slumping and/or sliding process, and (2) an clear predominance of tabular bodies with wide lateral continuity. The homoclinal ramp can be developed in epeiric platforms, mostly in a cratonic area (e.g., Shaw, 1964). Firstly, this ramp has been developed under humid climatic settings; factor suggested due to a less erosion verified, and probably associates to rapidly rising sea-level conditions (e.g., Strasser, 2015). It's that, therefore, not allowed a best developed of facies with subaerial exposure structures. Posteriorly, this development occurs under an arid climate, which is evidenced by a significant presence of facies- rich pseudomorphs after evaporates, as well as by the presence of facies containing subaerial exposure features (F3).

\subsection{SEQUENCES HIERARCHY}

Main hierarchical systems for stratigraphic sequences classifications was build upon dividing sequences of different orders of magnitude by the time interval attributed to their development (Catuneanu et al. 2009; 2011). Six distinct orders were recognized, from the 1st order, the longest time interval (hundreds of millions of years) of the depositional record, to successively shorter time intervals until the 6th order (the shortest). However, due to limitations in the application of such classification based on a precise geochronological framework, especially to Proterozoic aged strata, researchers must classify the stratigraphic sequence orders based on their recurrence in the geological record, as discussed in 


\section{Large-scale sequences - Incomplete, ca. $80 \mathrm{~m}$}

\section{Medium-scale sequences C-type}

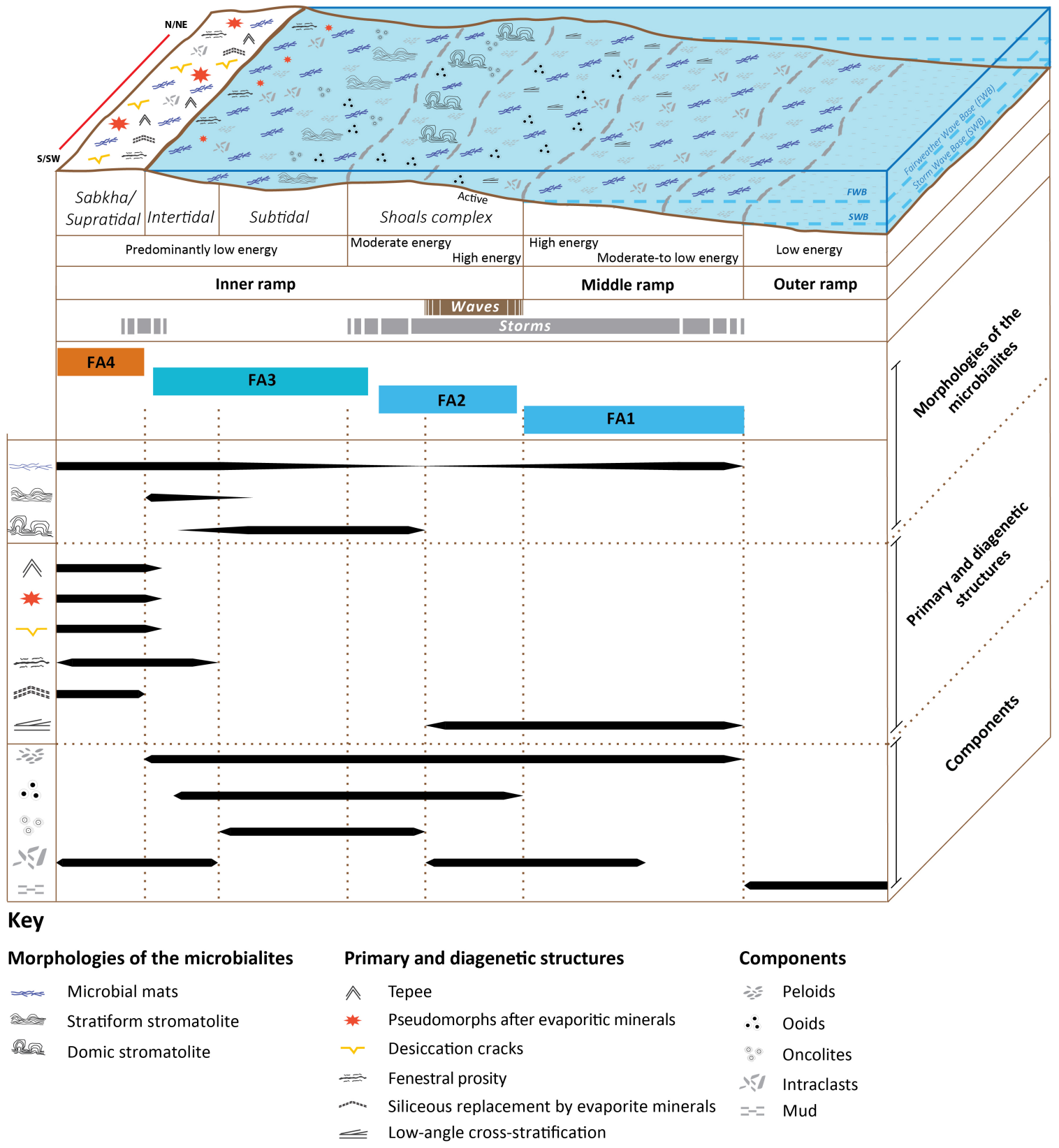

Figura 20. General ramp model and facies associations' distributions. Subdivision in accordance with Burchette and Wright, 1992. 
Catuneanu et al. (2009) and Catuneanu et al. (2011). Using this concept, sequences can be classified in relative terms on the frequency it occurs. Thus, lower order sequences are strongly influenced by processing influencing for longer times, as the complete filling of a sedimentary basin; while higher order sequences are controlled by shorter time processes, as orbital to suborbital driven climatic changes. Figure 19 shows the correspondence between different orders and frequencies, from higher to lower. Several authors have argued that each one of these stratigraphic orders is related to a particular control, either tectonic and/or climatic, being climatic control attributed to the 4th to 6 th order, as together with the 3rd order, have duration similar to orbital to suborbital cycles (Milankovitch and minor cycles). Other cycles, with a higher frequency of occurrence in the geological record and also responsible for variations in the quantity of heat received by the Earth are millennial-scale cycles as defined by Tucker et al. (2009). These cycles can define the very high-frequency order $\left(6^{\text {th }}\right.$ order).

In the study area, sequences occur in an obvious ordering: elementary sequences compose lower order sequences that edify medium scale sequences. The more recurrent the geological record, the higher the frequency, and the sequence will be a high-resolution candidate. Thus, as verified earlier, elementary and small-scale sequences are the natural candidates to appear as sequences of higher resolution, corresponding respectively to the 6th and 5th order. This simplification for the hierarchy of the different sequences, although plausible and unique because they are Neoproterozoic rocks, is also supported by the comparison between the data of the present research and studies developed in similar 
carbonaceous sequences of the Phanerozoic that have a large collection of geochronological data, biostratigraphic, among others, that allow the insertion in the hierarchical classification constructed from temporal variation.

Thus, sequences A and B-type, 6th and 5th order, respectively, are the most important for a better understanding of the flow lines of a similar reservoir, or control of primary mineralization, since their limits tend to be constituted by important permo-porous zones constituted By facies with diagenetic features of exposure, such as F3 facies, for example, which commonly exhibits canal and vugular porosities.

The inference of the controlling factors for the formation of the different sequences identified in the research also follows an analogous reasoning: sequences of higher frequency, elementary sequences, may be reflecting millennial-scale cycles of the 5th order (small-scale) sequence. They are generated from the conjunction of orbital cycles of precession and obliquity, while those with the lowest relative frequency, 4th order - mean scale sequences -, and should represent the eccentricity response. As postulated by several authors (Strasser et al., 1999; Freire, 2012; and others), the eccentricity cycles should reflect more gradual changes in the climate and, consequently, in the register of the sequences of 4a order, medium scale.

However, the $5^{\text {th }}$ order small-scale sequences usually show abrupt changes in facies that possibly reflect the interaction between the precession and obliquity cycles that imply faster changes in the position of the Earth's axis of rotation. Analogous to the well-studied 
sequences in the Phanerozoic, it is possible to admit that the control for the formation of a certain sequence, within its scale of observation, was governed by the same processes.

\subsection{CONCLUSIONS}

A integrate study was carried out within the microbial-dominated carbonate ramp of the intermediate to the superior portion of Unit B, Salitre Formation, Brazil. Ten facies and 8 subfacies were characterized. The vertical succession of these facies allowed the definition of four distinct facies associations (FAs): Middle ramp with microbial influence (FA1); Shallow inner to the proximal middle ramp (FA2); Inner ramp microbial-dominated (FA3); Subaerial ramp/Sabkha (FA4). Facies associations described to a typical framework of shallow marine carbonate developed in a homoclinal carbonate ramp. Firstly, this ramp has been developed under humid climatic settings; after, under an arid climate.

Sequential analysis at high resolution distinguished elementary and small scale sequence within intervals with a predominance of the FA3 and FA4. Elementary sequences (A-type) show typical thicknesses of the few tens of centimetres. These sequences were delineated in the field mainly after the identification of orderly factors series, such facies or subfacies that by vertical succession demonstrated a periodicity/variability of the microbial growth. Smallscale sequences (B-type) are constituted by elementary sequences. Their demarcation occurred from sedimentological criteria and aid of the gamma ray log. 
Medium-scale sequences were delineated from the FA changes; implies in vertical succession between the different FA types, antagonistic in proximity or distality, in relation to the coastline. The large-scale sequence was inferred and shown tens of meters thick. This sequence suggests a more significant cyclical change of environmental conditions. However, we believe that a framework of medium and large-sequence will be best delineated only from regional study employees with the same detail and method as implemented here.

The high-resolution stratigraphic analysis method in Neoproterozoic marine microbial carbonate systems was improved with the pioneering integration of conventional data (sedimentological and petrographic) and spectral gamma-ray log. Thus, this work provided criteria that contribute to the development of predictive models in Neoproterozoic microbial carbonates that applied to the areas with mineral occurrence into the basin. for example, could imply in greater traceability of porosity and permeability zones, commonly associated with diagenetic processes.

At the petroleum industry, sequence framework at high resolution often may be made parameters for a better understanding of the flow lines in reservoirs in microbialite domain, or even in other reservoirs where there is a clear cyclic control, establishing the generation of different facies/diagenetic features, such as variation in the base level. In view of the greater random character of the carbonates facies, especially in depositional contexts where shallow water conditions prevail, the analysis from the perspective of high-frequency stratigraphy is fundamental and provides predictive parameters for successful exploitations. 


\section{Acknowledgements}

This work is part of the doctoral project carried out by the first author, and also is part of a research project on carbonates, which represents a collaboration of the UFRGS and PETROBRAS ("Microbial Project"). We thank Brazilian Research Agency - CNPq (grant 160391/2011-3) and PETROBRAS/Brazil for their financial support. We thank Cícero da Paixão Pereira (Universidade Federal da Bahia), geologists of PETROBRAS University, and undergraduate and graduate students for their assistance in the field and/or processing data. Fora da Trilha Agency, Lapa Doce, and Torrinha Cave staffs are thanked for providing field assistance. F. Guadagnin is acknowledged for constructive comments.

\section{References}

Aigner, T., Schauer, M., Junghans, W.D., Reinhardt, L., 1995. Outcrop gamma-ray logging and its applications: examples from the German Triassic. Sediment. Geol. 100, 47-61. doi:10.1016/0037-0738(95)00102-6

Alkmim, F.F., Marshak, S., Pedrosa-Soares, A.C., Peres, G.G., Cruz, S.C.P., Whittington, A., 2006. Kinematic evolution of the Araçuaí-West Congo orogen in Brazil and Africa: Nutcracker tectonics during the Neoproterozoic assembly of Gondwana. Precambrian Res. 149, 43-64. doi:10.1016/j.precamres.2006.06.007

Amour, F., Mutti, M., Christ, N., Immenhauser, A., Agar, S.M., Benson, G.S., Tom??s, S., Alway, R., Kabiri, L., 2012. Capturing and modelling metre-scale spatial facies heterogeneity in a Jurassic ramp setting (Central High Atlas, Morocco). Sedimentology 59, 1158-1189. doi:10.1111/j.1365-3091.2011.01299.x

Argenio, B., Ferreri, V., Amodio, S., Pelosi, N., 1997. Hierarchy of high-frequency orbital 
cycles in Cretaceous carbonate platform strata. Sediment. Geol. 113, 169-193. doi:10.1016/S0037-0738(97)00076-6

Assereto, R. L., \& Kendall, C. G. , 1977. Nature, origin and classification of peritidal tepee structures and related breccias. Sedimentology. 24(2), 153-210.

Awramik S.M.,; Benmore W.C.., 1979, Stromatolite morphogenesis-Progress and problems: Canadian Journal of Earth Sciences, v. 16, p

Badenas, B., Aurell, M., Bosence, D., 2010. Continuity and facies heterogeneities of shallow carbonate ramp cycles (Sinemurian, Lower Jurassic, North-east Spain). Sedimentology 57, 1021-1048. doi:10.1111/j.1365-3091.2009.01129.x

Bahniuk, A.M., Anjos, S., França, A.B., Matsuda, N., Eiler, J., Mckenzie, J.A., Vasconcelos, C., 2015. Development of microbial carbonates in the lower cretaceous Codó Formation (north-east Brazil): Implications for interpretation of microbialite facies associations and palaeoenvironmental conditions. Sedimentology 62, 155-181. doi:10.1111/sed.12144

Barbosa, J.S.F., Sabaté, P., 2004. Archean and Paleoproterozoic crust of the São Francisco Craton, Bahia, Brazil: Geodynamic features. Precambrian Res. 133, 1-27. doi:10.1016/j.precamres.2004.03.001

Bento-Freire, E., 2012 Caracterização estratigráfica em alta resolução das sequências calcárias de origem microbiana do intervalo paleocênico da Formação Yacoraite (Sequência Balbuena IV) na região de Salta - Argentina. Master thesis, Universidade Federal do Rio de Janeiro, Instituto de Geociências, X p.

Bialik, O.M., Korngreen, D., Benjamini, C., 2012. Lithofacies and cyclicity of Mohilla evaporite basins on the rifted margin of the Levant in the Late Triassic, Makhtesh Ramon, southern Israel. Sedimentology 59, 2097-2124. doi:10.1111/j.1365-3091.2012.01336.x 
Bomfim, L. F. C., Rocha, A. J. D., Pedreira, A. J., MoraisFilho, J. C., Guimarães, J. T., Tesch, N. A. , 1985. Projeto Bacia de Irecê, Relatório Final. Salvador: CPRM.

Bosence D.W.J., Prokter E., Aurell, M., Kahla, A.B., Boudaghjer-Fadel, M., Casaglia, F., Cirilli, S., Mehdie, M., Nieto, L., Rey, J., Scherreiks, R., Soussi, M., Waltham, D., 2009.A dominant tectonic signal in high-frequency, peritidal carbonate cycles? A regional analysis of Liassic platforms from western Tethys. Journal of Sedimentary Research 79, 389-415.

Branner, J.C., 1911. The papers of Stanford Expedition to Brazil.

Palo Alto: Stanford University.

Burchette, T.P., Wright, V.P., 1992. Carbonate ramp depositional systems. Sediment. Geol. 79, 3-57. doi:10.1016/0037-0738(92)90003-A

Burne, R.V., Moore, L.S., 1987. Microbialites: Organosedimentary deposits of benthic microbial communities. Palaios 2, 241-254.

Catuneanu, O., Abreu, V., Bhattacharya, J. P., Blum, M. D., Dalrymple, R. W., Eriksson, P. G., ... \& Giles, K. A., 2009. Towards the standardization of sequence stratigraphy. EarthScience Reviews, 92(1), 1-33.

Catuneanu, O., Bhattacharya, J. P., Blum, M. D., Dalrymple, R. W., Eriksson, P. G., Fielding, C. R., ... \& Giles, K. A., 2010. Thematic Set: Sequence stratigraphy: common ground after three decades of development. First break, 28(1), 41-54.

Catuneanu, O., Galloway, W.E., Kendall, C.G.S.C., Miall, A.D., Posamentier, H.W., Strasser, A., Tucker, M.E., 2011. Sequence Stratigraphy: Methodology and Nomenclature. Newsletters Stratigr. 44, 173-245. doi:10.1127/0078-0421/2011/0011

Caxito, F., Halverson, G. P., Uhlein, A., Stevenson, R., Dias, T. G., \& Uhlein, G. J., 2012. 
Marinoan glaciation in east central Brazil. Precambrian Research, 200, 38-58.

Chemale Jr., F., Alkmim, F.F., Endo, I., 1993. Late Proterozoic tectonism in the interior of theSão Francisco craton. In: Findlay, R.H., Unrug, R., Banks, M.R., Veevers, J.J. (Eds.), Gondwana Eight: Assembly, Evolution and Dispersal. Balkema, Rotterdam, 29-41.

Choquette, P. W. and Pray, L. C., 1970. Geologic nomenclature and classification of porosity in sedimentary carbonates. AAPG bulletin, 54(2), 207-250.

Cruz, S.C.P., Alkmim, F.F., 2006. The tectonic interaction between the Paramirim Aulacogen and the Araçuaí Belt, São Francisco craton region, Eastern Brazil. An. Acad. Bras. Cienc. 78, 151-173. doi:/S0001-37652006000100014

Dalrymple, R.W., 2010. Tidal depositional systems. In: James, N.P., Dalrymple, R.W. (Eds.), Facies Models 4. Geological Association of Canada, pp. 201-231.

Dardenne, M.A., 1978. Síntese sobre a estratigrafia do Grupo Bambuí no Brasil Central, in: Congresso Brasileiro de Geologia. pp. 597-610.

Demmico and Hardie, 1994. Sedimentary Structures and Early Diagenetic Features of Shallow Marine Carbonate Deposits. SEPM (Society of Sedimentary Geology), Universidade de Michigan, 265 pp.

Duarte, K.S., Vasconcellos, R.V.A., Rugenski, A., 2012. Contribuição da Geofísica na definição do potencial petrolífero da Bacia do Irecê. pp. 1-3.

Dumas, S., Arnott, R.W.C., 2006. Origin of hummocky and swaley cross-stratification- the controlling influence of unidirectional currentstrength andaggradation rate. Geology 34, 1073-1076

Dunham, R.J., 1962. Classification of Carbonate Rocks According to Depositional Texture. In: Ham, W.E., Ed., Classification of Carbonate Rocks, AAPG, Tulsa, 108-121. 
Dunn, P.R., Thomson, B.P., Rankama, K., 1971. Late Pre-Cambrian Glaciation in Australia as a stratigraphic boundary. Nature 231, 498-502.doi: 10.1038/231498a0

Dupraz, Christophe, et al., 2009. Processes of carbonate precipitation in modern microbial mats. Earth-Science Reviews, 96.3: 141-162.

Dibenedetto, S., Grotzinger, J., 2005. Geomorphic evolution of a storm-dominated carbonate ramp ( c. 549 Ma), Nama Group, Namibia. Geol. Mag. 142, 583. doi:10.1017/S0016756805000890

El Tabakh, M., Riccioni, R., SCHREIBER, B. CHARLOTTE. Evolution of late Triassic rift basin evaporites (Passaic Formation): Newark Basin, Eastern North America. Sedimentology, 1997, 44.4: 767-790.

Embry, A. F., Johannessen, E. P., 1992. T-R sequence stratigraphy, facies analysis and reservoir distribution in the uppermost Triassic-Lower Jurassic succession, western Sverdrup Basin, Arctic Canada. In: Vorren, T. O., Berg - sager, E., Dahl-Stamnes, O. A., Holter, E., Johansen, B., Lie, E., Lund, T. B. (Eds.), Arctic Geology and Petroleum Potential, vol. 2 (Special Publication). Norwegian Petroleum Society (NPF), pp. 121-146. Embry, A. F., \& Klovan, J. E. (1971). A late Devonian reef tract on northeastern Banks Island, NWT. Bulletin of Canadian Petroleum Geology, 19(4), 730-781.

Figueiredo, F.T., 2008. Fácies sedimentares e proveniência da Formação Bebedouro, Neoproterozóico (BA). Master thesis, Universidade de São Paulo, Instituto de Geociências, 121 p.

Figueiredo, F.T., Almeida, R.P., Tohver, E., Babinski, M., Liu, D., Fanning, C.M., 2009. Neoproterozoic glacial dynamics revealed by provenance of diamictites of the Bebedouro Formation, São Francisco Craton, Central Eastern Brazil. Terra Nov. 21, 375- 
385. doi:10.1111/j.1365-3121.2009.00893.x

Flügel, E., 2010. Microfacies of Carbonate Rocks: Analysis, Interpretation and Application. doi:10.1007/978-3-642-03796-2

Grotzinger, J.P., 1986. Upward shallowing platform cycles: A response to 2.2 billion years of low-amplitude, high-frequency (Milankovitch band) sea level oscillations. Paleoceanography 1, 403-416. doi:10.1029/PA001i004p00403

Grotzinger, J., Al-Rawahi, Z., 2014. Depositional facies and platform architecture of microbialite-dominated carbonate reservoirs, ediacaran-cambrian ara group, sultanate of Oman. Am. Assoc. Pet. Geol. Bull. 98, 1453-1494. doi:10.1306/02271412063

Guadagnin, F., Chemale Junior, F., Magalhães, A.J.C., Alessandretti, L., Bállico, M.B., Jelinek, A.R., 2015b. Sedimentary petrology and detrital zircon U-Pb and Lu-Hf constraints of Mesoproterozoic intracratonic sequences in the Espinhaço Supergroup: Implications for the Archean and Proterozoic evolution of the São Francisco Craton. Precambrian Res. 266, 227-245. doi:10.1016/j.precamres.2015.05.027

Guimarães, J.T., 1996. A Formação Bebedouro no Estado da Bahia: Faciologia , Estratigrafia e Ambientes de Sedimentação. Universidade Federal da Bahia, Instituto de Geociências, $146 p$.

Guimarães, J. T.; Alkmim, F. F.; Cruz, S. C. P., 2012. In: Barbosa, J. S. (Coord.), Geologia da Bahia, Pesquisa e Atualização. CBPM, Série Publicações especiais, Salvador, Brazil, 3385.

Halverson, G.P., Shields-Zhou, G., 2011. Chapter 4 Chemostratigraphy and the Neoproterozoic glaciations. Geol. Soc. London, Mem. 36, 51-66.

Karfunkel, J., Hoppe, A., 1988. Late Proterozoic glaciation in central-eastern Brazil: Synthesis 
and model. Palaeogeogr. Palaeoclimatol. Palaeoecol. 65, 1-21. doi:10.1016/00310182(88)90108-3

Labaj, M.A., Pratt, B.R., 2016. Depositional dynamics in a mixed carbonate-siliciclastic system:

Middle-Upper Cambrian Abrigo Formation, southeastern Arizona, U.S.A. J. Sediment. Res. 86, 11-37. doi:10.2110/jsr.2015.96

Leão, Z.M.A.N. \& Dominguez, J.M.L. 1992. Plataformas carbonáticas pré-cambrianas: o exemplo da Formação Salitre, Proterozóico Superior, Estado da Bahia. Rev Bras de Geoc. 22(4):407-421.

Logan, B. W., Rezak, R., \& Ginsburg, R. N., 1964. Classification and environmental significance of algal stromatolites. The Journal of Geology, 72(1), 68-83.

Loureiro, H.S.C., Lima, E.S., Macedo, E.R., Silveira, F.V., Bahiense, I.C., Arcanjo, J.B.A., Moraes Filho, J.C., Neves, J.P., Guimarães, J.T., Teixeira, L.R., Abram, M.B., Santos, R.A., Melo,R.C., 2008. Projeto Barra-Oliveira dos Brejinhos Geological map. Brazilian Geological Survey and Bahia Mineral Research Company, scale 1:200.000.

Lucia, F. J. , 2007. Carbonate reservoir characterization: an integrated approach. Springer Science \& Business Media.

Magalhães, A. J.C., Raja Gabaglia, G.P., Scherer, C.M.S., Bállico, M.B., Guadagnin, F., Bento Freire, E., Silva Born, L.R., Catuneanu, O., 2015. Sequence hierarchy in a Mesoproterozoic interior sag basin: from basin fill to reservoir scale, the Tombador Formation, Chapada Diamantina Basin, Brazil. Basin Res. n/a-n/a. doi:10.1111/bre.12117

Misi, A., Kaufman, A.J., Azmy, K., Dardenne, M.A., 2011. Neoproterozoic successions of the Sao Francisco Craton , Brazil: the Bambuí, Una, Vazante and Vaza Barris / Miaba groups 
and their glaciogenic deposits. Geol. Rec. Neoproterozoic Glaciat. 36, 509-522. doi:10.1144/M36.48

Misi, A., Kaufman, A.J., Veizer, J., Powis, K., Azmy, K., Boggiani, P.C., Gaucher, C., Teixeira, J.B.G., Sanches, A.L., Iyer, S.S.S., 2007. Chemostratigraphic correlation of Neoproterozoic successions in South America. Chem. Geol. 237, 161-185. doi:10.1016/j.chemgeo.2006.06.019

Misi, A., Kyle, J.R., 1994. Upper Proterozoic carbonate stratigraphy, diagenesis, and stromatolite phosphorite formation, Irece Basin, Bahia, Brazil. J. Sed. Res. A64, 299-310.

Misi, A., Veizer, J., 1998. Neoproterozoic carbonate sequences of the Una Group, Irecê Basin, Brazil: chemostratigraphy, age and correlations. Precambrian Res. 89, 87-100. doi:10.1016/S0301-9268(97)00073-9

Misi, A., 1979. O Grupo Bambuí no Estado da Bahia. In: Inda, H.V. (Ed.), Geologia e Recursos Minerais do Estado da Bahia, Textos Básicos. Salvador, Brazil, 120-54.

Misi, A., Kaufman, A.J., Azmy, K., Dardenne, M.A., 2011. Neoproterozoic successions of the Sao Francisco Craton, Brazil : the Bambuı, Una, Vazante and Vaza Barris / Miaba groups and their glaciogenic deposits. Geol. Rec. Neoproterozoic Glaciat. 36, 509-522. doi:10.1144/M36.48

Misi, A., Kaufman, A.J., Veizer, J., Powis, K., Azmy, K., Boggiani, P.C., Gaucher, C., Teixeira, J.B.G., Sanches, A.L., Iyer, S.S.S., 2007. Chemostratigraphic correlation of Neoproterozoic successions in South America. Chem. Geol. 237, 161-185. doi:10.1016/j.chemgeo.2006.06.019

Misi, A., Kyle, R., 1994. Upper Proterozoic carbonate stratigraphy, diagenesis, and stromatolitic phosphorite formation, Irecê basin, Bahia, Brazil. J. Sediment. Res. A64, 
299-310. doi:1073-130X/94/0A64-299

Misi, A., Veizer, J., 1998. Neoproterozoic carbonate sequences of the Una Group, Irecê Basin, Brazil: chemostratigraphy, age and correlations. Precambrian Res. 89, 87-100. doi:10.1016/S0301-9268(97)00073-9

Misi, A., Souto, P.G., 1975. Controle estratigráfico das mineralizações de Pb-Zn-F-Ba do Grupo Bambui, parte leste da Chapada de Irecê (Bahia). Rev. Bras. Geociências, 5, 3045.

Montanez, I. P., \& Read, J. F., 1992. Fluid-rock interaction history during stabilization of early dolomites, upper Knox Group (Lower Ordovician), US Appalachians. Journal of Sedimentary Research, 62(5).

Muniz, M.C., Bosence, D.W.J., 2015. Pre-salt microbialites from the Campos Basin (offshore Brazil): image log facies, facies model and cyclicity in lacustrine carbonates. Geol. Soc. London, Spec. Publ. 418, 221-242. doi:10.1144/SP418.10

Noffke, N., Gerdes, G., \& Klenke, T., 2003. Benthic cyanobacteria and their influence on the sedimentary dynamics of peritidal depositional systems (siliciclastic, evaporitic salty, and evaporitic carbonatic). Earth-Science Reviews, 62(1), 163-176.

Noffke, N., Gerdes, G., Klenke, T., \& Krumbein, W. E. (2001). Microbially Induced Sedimentary Structures--A New Category within the Classification of Primary Sedimentary Structures: PERSPECTIVES. Journal of Sedimentary Research, 71(5), 649656.

Pawellek, T., Aigner, T., 2004. Dynamic stratigraphy as a tool in economic mineral exploration: ultra-pure limestones (Upper Jurassic, SW Germany). Mar. Pet. Geol. 21, 499-516. 
Pedreira, A.J.C.L., Oliveira, J.E., Silva, B.C., Pedrosa, C.J., 1975. Projeto Bahia. Relatório final. CPRM, Salvador (unpublished)

Riding, R., 2000. Microbial carbonates : the geological record of calcified bacterial-algal mats and biofilms. Sedimentology 47, 179-214. doi:10.1046/j.1365-3091.2000.00003.x

Rooney, A.D., Strauss, J.V., Brandon, A.D., Macdonald, F.A., 2015. A Cryogenian chronology: Two long-lasting synchronous Neoproterozoic glaciations. Geology 43, 459-462. doi:10.1130/G36511.1

Santana, A.V.A,, 2016. Análise estratigráfica em alta resolução em rampa carbonática dominada por microbialitos, Formação Salitre, Bacia d Irecê, Bahia. Thesis, Universidade de Brasília, Instituto de Geociências, 205 p.

Santana, A. V.A., Chemale Jr, F., Scherer, C., Guadagnin, F., Pereira, C. (in prep.) New insights of gulf carbonate sedimentation of the Irecê Basin, São Francisco Craton, based on stratigraphic analyses and isotope studies. Submitted to Journal of South American Earth Sciences.

Shaw, A.B., 1964. Time in Stratigraphy. McGraw-Hill, New York, N.Y., 365 pp.

Shin, E.A., 1983. Tidal flat environments. American Association of Petroleum Geologists Memoir 33:172-210.

Silveira, E.M., Söderlund, U., E.P. Oliveira, R.E. Ernst, Menezes Leal, A.B., 2013. First precise U-Pb baddeleyite ages of 1500 Ma mafic dykes from the São Francisco Craton, Brazil, and tectonic implications Lithos, 174, 144-156

Simonson, B.M., Carney, K.E., 1999.Roll-up structures: Evidence of in situ microbial mats in Late Archean deep shelf environments Palaios, 14, 13-24 
Souza, J. D., Kosin, M.,; Melo, R. C., Santos, R. A., Teixeira, L. R., Sampaio, A. R., Guimarães, J. T., Vieira Bento, R., Borges, V. P., Martins, A. A. M., Arcanjo, J. B., Loureiro,H. S. C., Angelim, L. A. A., 2003. Mapa Geológico do Estado da Bahia - Escala 1:1.000.000. Salvador: CPRM, 2003. Versão 1.1. Programas Carta Geológica do Brasil ao Milionésimo e Levantamentos Geológicos Básicos do Brasil (PLGB). Convênio de Cooperação e Apoio Técnico-Científico CBPM-CPRM.

Souza, S.L., Brito, P.C.R., Silva, R.W.S., 1993. Estratigrafia, Sedimentologia e Recursos Minerais da Formação Salitre na Bacia de Irecê, Bahia. Integração e Síntese por Augusto J. Pedreira. CBPM, Salvador (34 pp.)

Strasser, A., 2015. Hiatuses and condensation: an estimation of time lost on a shallow carbonate platform. Depos. Rec. 1, 91-117. doi:10.1002/dep2.9

Strasser, A., Pittet, B., Hillgärtner, H., Pasquier, J.B., 1999. Depositional sequences in shallow carbonate-dominated sedimentary systems: Concepts for a high-resolution analysis. Sediment. Geol. 128, 201-221. doi:10.1016/S0037-0738(99)00070-6

Strasser, A., Védrine, S., Stienne, N., 2012. Rate and synchronicity of environmental changes on a shallow carbonate platform (Late Oxfordian, Swiss Jura Mountains). Sedimentology 59, 185-211. doi:10.1111/j.1365-3091.2011.01236.x

Teles, G., Chemale, F., dthese Oliveira, C.G., 2015. Paleoarchean record of the detrital pyritebearing, Jacobina Au-U deposits, Bahia, Brazil. Precambrian Res. 256, 289-313. doi:10.1016/j.precamres.2014.11.004

Terra, J.G.S., Spadini, A.R., França, A.B., Sombra, C.L., Zambonato, E.E., Juschaks, L.C.S., Arienti, L.M., Erthal, M.M., Blauth, M., Franco, M.P., Matsuda, N.S., Goulart, N., Moretti Junior, P.A., D’Ávila, R.S.F., Souza, R.S., Tonietto, S.N., Anjos, S.M.C., Campinho, V.S., 
Winter, W.R., 2010. Classificação de rochas carbonáticas aplicável às bacias sedimentares brasileiras. B. Geoci. Petrobras, Rio Janeiro 18, 9-29.

Torquato, J.R., Misi, A., 1977. Medidas Isotópicas de Carbono e Oxigênio em Carbonatos do Grupo Bambuí, na Região Centro-Norte do Estado da Bahia. Rev. Bras. Geociências 7, $14-24$

Tucker, M. E., 1976. Replaced evaporites from the late Precambrian of Finnmark, arctic Norway. Sedimentary Geology, 16(3), 193-204.

Tucker and Wright, 1990. Carbonate Sedimentology. Blackwell, Oxford, 482 pp.

Zhou, C., Tucker, R., Xiao, S., Peng, Z., Yuan, X., Chen, Z., 2004. New constraints on the ages of Neoproterozoic glaciations in south China. Geology 32, 437-440. doi:10.1130/G20286.1 


\title{
5 NEW INSIGHTS OF GULF
}

\section{CARBONATE SEDIMENTATION OF THE IRECÊ BASIN, SÃO FRANCISCO CRATON, BASED ON STRATIGRAPHIC ANALYSES AND ISOTOPE STUDIES}

\author{
Ana Santana ${ }^{a, b, c}$; Farid Chemale Júnior ${ }^{a}$; Claiton Scherer ${ }^{b}$; Felipe Guadagnin ${ }^{c}$; Cícero \\ Pereira $^{d}$
}

Instituto de Geociências, Universidade de Brasília, Campus Universitário, Asa Norte, 70910-900, Brasília, DF, Brazil; ana.santana.geo@gmail.com

$b \quad$ Petroleum Geology Research Group, Instituto de Geociências, Universidade Federal do Rio Grande do Sul, Campus do Vale, Av. Bento Gonçalves 9500, 91509-900, Porto Alegre, RS, Brazil.

c Sedimentary Geology Research Group, Universidade Federal do Pampa, Campus de Caçapava do Sul, Av. Pedro Anunciação 111, 96570-000, Caçapava do Sul, RS, Brazil.

d Instituto de Geociências, Universidade Federal da Bahia, Campus de Ondina, Rua Barão de Jeremoabo, 147, 40170-115, Salvador, BA, Brazil. 
01/03/2017 Homepage

Ref: SAMES_2016_146

Title: New insights of gulf carbonate sedimentation of the Irecê Basin, São Francisco Craton, based on stratigraphic analyses and isotope studies Journal: Journal of South American Earth Sciences Dear Dr. Chemale Junior,

Thank you for submitting your manuscript to Journal of South American Earth Sciences. We have completed the review of your manuscript. A summary is appended below. While revising the paper please consider the reviewers' comments carefully. We look forward to receiving your detailed response and your revised manuscript.

To submit your revised manuscript:

Log into EVISE $^{\circledR}$ at:

http://www.evise.com/evise/faces/pages/navigation/NavController.jspx?JRNL_ACR=SAMES

Locate your manuscript under the header 'My Submissions that need Revisions' on your 'My

Author Tasks' view Click on 'Agree to Revise'

Make the required edits

Click on 'Complete Submission' to approve

What happens next?

After approving your submission you will receive a notification that the submission is complete. To track the status of your paper throughout the editorial process, log into EVISE ${ }^{\circledR}$ at: http://www.evise.com/evise/faces/pages/navigation/NavController.jspx?JRNL_ACR=SAMES

Enrich your article to present your research with maximum impact. This journal supports the following Content Innovations:

Explain your research in your own words and attract interest in your work using AudioSlides : 5-minute webcast-style presentations that are displayed next to your published article and can be posted on other websites. You will receive an invitation email to create an AudioSlides presentation within three weeks after your paper has been accepted.

Interactive Map Viewer: Interactive viewer displaying geospatial data on a Google Map . Please use the online KML/KMZ Editor or your own geographic information system (GIS) to create the file required for uploading. GIS users can preview how files will display here.

Interactive Plots: Interactive plot viewer providing easy access to the data behind plots. Please prepare a .CSV file with your plot data and test it online here before submitting as supplementary material.

I look forward to receiving your revised manuscript as soon as possible. Kind regards, Professor Fuck Regional Editor

Journal of South American Earth Sciences https://www.evise.com/evise/faces/pages/homepage/homepage.jspx?_adf.ctrl-state=16j7tba3yo $4 \quad 1 / 3$ 


\section{Abstract}

Neoproterozoic carbonate sequence overlying the Middle Cryogenian glacial deposits of the Sao Franscico Craton (CSF) in the central region of Bahia is investigated to provide information on the stratigraphy framework, the maximum age of deposition and possible source areas for the stratigraphic Unit B of the Salitre Formation, southern region of the syncline of Irecê. Based on stratigraphic analyses, in the basal and intermediate section of Unit B were characterized three different depositional zones: basin, outer ramp, and middle ramp settings. Isotope $(C, O)$ data obtained show slightly negative $\delta{ }^{13} \mathrm{C}$ values, close to $0.0 \%$, and suggest a typical ocean isotopic signature. U-Pb SHRIMP zircon analyses were carried out in tuffacceous siltstone (tuffite) and rock with significant epiclastic contribution. The maximum depositional age of Salitre Formation is restricted by the age of the youngest zircon, close to $670 \mathrm{Ma}$, obtained from the tuffite, which it can be associated with distal magmatism in the passive margin or active margin of the surrounding Brasiliano mobile belts. The obtained detrital zircon age distribution of carbonate platform is marked by peaks at 3.38, 2.56 and $2.07 \mathrm{Ga}$ at the base of Unit B, and in the intermediate section of the Unit B contain not only Archean and Paleoproterozic zircon grains but also Mesoproterozoic ones with peaks at 1.78 and $1.50 \mathrm{Ga}$. Older zircon detrital ages are mainly from local sources such as granite-gneiss rocks and greenstone belts associated with the Gavião Block (> 2.0 Ga; CSF basement in the study area), where the younger one is associated with volcanic events preserved in the Espinhaco Group. The zircon age distribution of the Unit B and sedimentary record suggests intra-Unit B unconformity, indicates by change detrital zircon age patterns. Our results point the Irece Basin formed in a gulf-like basin as extension of the Neoproterozoic passive margin at the end of the Sturtian glaciation.

Keywords: Neoproterozoic gulf basin; U-Pb SHRIMP zircon dating; carbonate platform; Irecê Basin; São Francisco Craton. 


\subsection{INTRODUCTION}

The presence of at least three low-latitude glaciations in the Neoproterozoic Era, MiddleCryogenian (ca. 717-660 Ma; Halverson and Zhou, 2011; Rooney et al., 2015), EndCryogenian, or Marinoan event (ca. 635 Ma; Dunn et al., 1971; Halverson and Zhou, 2011) and Gaskiers event (ca. 580 Ma; Knoll et al., 2004) which are bounded with the later diversification of animal life after the youngest glaciation, represents a singular event in Earth's history. Similar features are found in each glacial cycle worldwide, such as banded iron formations, cap carbonates with negative $\delta^{13} \mathrm{C}$ anomalies covering the glacial deposits and $\delta^{13} \mathrm{C}$ positive excursion in the upper carbonate platform deposits (Hoffmann et al., 1998). Neoproterozoic aged carbonate sequences overlying widespread glacial deposits are well preserved the São Francisco Craton as part of the São Francisco Supergroup (e.g., Misi et al., 2011). Those sequences crop out in two distinct basins: (1) the Irecê Basin, which it comprises here the Irecê, Una-Utinga, Salitre and Ituaçu synclines, in the central portion of the craton within the Paramirim Aulacogen (Figs. 21B and C); and (2) the São Francisco Basin, in the western portions of the craton (e.g. Dardenne, 1978; Misi, 1979; Misi et al., 2007). These sequences are marked by the presence of the glacial deposits, cap carbonate with negative $\delta^{13} \mathrm{C}$ excursion and overlying carbonate platform with positive $\delta^{13} \mathrm{C}$ excursion (Misi et al., 2007).

The São Francisco Basin is characterized by two major sequences, the basal one is composed by glacial deposits of the Jequitaí Formation/Macaúbas Group and cap carbonate units dated at $740 \pm 22 \mathrm{Ma}(\mathrm{Pb}-\mathrm{Pb}$ whole-rock isochron; Babinski et al., 2007), deposited during the 
Sturtian glacial epoch ( 717-660 Ma; Halverson and Zhou, 2011; Rooney et al., 2015). Cap carbonates in this sequence correspond to the lowermost units of Sete Lagoas Formation, Bambuí Group (Dardenne, 1978; Vieira et al., 2007). Overlying sequences correspond to siliciclastic-carbonate platform of Sete Lagoas Formation. Middle portion of Sete Lagoas Formation presents maximum depositional age of $593 \pm 1.7 \mathrm{Ma}$, whereas upper portion presents maximum depositional age of ca. $557 \mathrm{Ma}$ (Paula-Santos et al., 2015). Warren et al. (2014) described also fossil assemblage Cloudina shells and very rare Corumbella werneri suggesting Late Ediacaran age to the deposition of Sete Lagoas Formation.

On other side, the age constrains of Irecê Basin are based mostly on ${ }^{87} \mathrm{Sr} /{ }^{86} \mathrm{Sr}$ ratios and C-O isotopes which point to a deposition between 750 to $600 \mathrm{Ma}$ for the glacial (Bebedouro Formation) and dominant carbonate platform (Salitre Formation) of the Una Group (Misi et al.,1998, 2007). Detrital U-Pb zircon data have been presented for the Bebedouro Formation with maximum depositional age of $874 \pm 9 \mathrm{Ma}$ and distribution age pattern of Archean, Paleoproterozoic and Mesoproterozoic (Figuereido et al., 2009). Santos et al. (2011) obtained detrital zircon ages of sandstones and arcosian sandstones rocks from Una Group units in the Salitre Syncline (Fig. 21 C), where the Bebedouro Formation, basal stratigraphic unit, and the lower section of the carbonate platform of the Salitre Formation, the upper stratigraphic unit, contains the youngest zircon of $1477 \pm 13 \mathrm{Ma}$ and $1703 \pm 15 \mathrm{Ma}$, respectively.

Indeed, the precise dating of deposition of Salitre Formation carbonates is hampered by the lack of index fossil and direct dating of deposition from intercalated volcanic sequences 
and/or minimum ages derived from reliable dating of diagenesis, intrusion, deformation and/or metamorphism (Misi et al., 1998) and the detrital zircon ages from the analyzed sections provide older ages as those obtained by isotope chemostratigraphy. On the other hand, volcanic and volcaniclastic rocks (tuff levels) intercalated with coupled diamictite-cap carbonate, which are characteristic of Neoproterozoic successions worldwide (Kennedy, 1996; Hoffman and Schrag, 2002), have been reported in East and West Gondwana (Frimmel et al., 1996; Key et al., 2001; Fanning, 2004; Fanning and Link, 2006; Hoffman et al., 2004; and others).

Nonetheless, during Gondwana supercontinent assembly, a series of orogenic systems developed in between the older continents. The assembly processes occurred through oceanic crust subduction and continental collision with related oceanic and continental arc magmatism mainly between ca. 880 and 600 Ma (e.g. Pimentel et al., 1999; Alkmim et al., 2006). This magmatic activity bordered São Francisco Craton and influenced sedimentation and/or served as source areas to the associated basins, such as the São Francisco basin (Pimentel et al., 2011; Paula-Santos et al., 2015). The Irecê Basin is bordered by the Rio Preto, Riacho do Pontal, Sergipana and Araçuaí mobile belts (Fig. 21 B). There is no record of juvenile volcanic rock (e.g., volcanogenic material such as tuffs or volcaniclastic sandstones) that could be connected with the main magmatic activity associated to the subduction processes of the Rio Preto, Riacho do Pontal, Sergipana mobile belts (Fig. 21 B). This magmatism associated to the subduction processes occurred between 640 Ma to 575 Ma (Pedrosa-Soares et al., 2008; Oliveira et al., 2010; Caxito and Uhlein, 2013). 
In order to understand the depositional ages and the contribution of magmatic and detrital zircon grains in the carbonate sequences of Salitre Formation, stratigraphic, geochemical and isotope studies were carried out in carbonate and associated rocks. Based on facies analysis of carbonate sequences in the Salitre Formation, it is recognized tuffite layers in the Salitre Formation basal section, deposited just above cap carbonates. U-Pb dating of detrital zircon grains allows the establishment of the maximum depositional age of this section and therefore defines the main sources of siliciclastic material to the carbonate platform. This study is conducted at detailed scale and was derived from field investigations in the Rio Preto Quarry, Chapada Diamantina region, central Bahia State, Brazil (UTM coordinates, South American Datum 1969, 24th zone: 219,013 mE; 8,622,856 mN; see Supplementary .kml file) which corresponds to lower Unit B of Salitre Formation (Misi and Souto, 1975; Misi, 1979). In addition, one detrital zircon sample from middle Unit B is used to compare the detrital zircon ages with published data from São Francisco Craton (Figueiredo et al., 2009; Guadagnin et al., 2015b) to unravel primary and secondary detrital zircon sources, understand the tectonic evolution of the Irecê Basin and its implications to the evolution of the western portion of Gondwana supercontinent. 

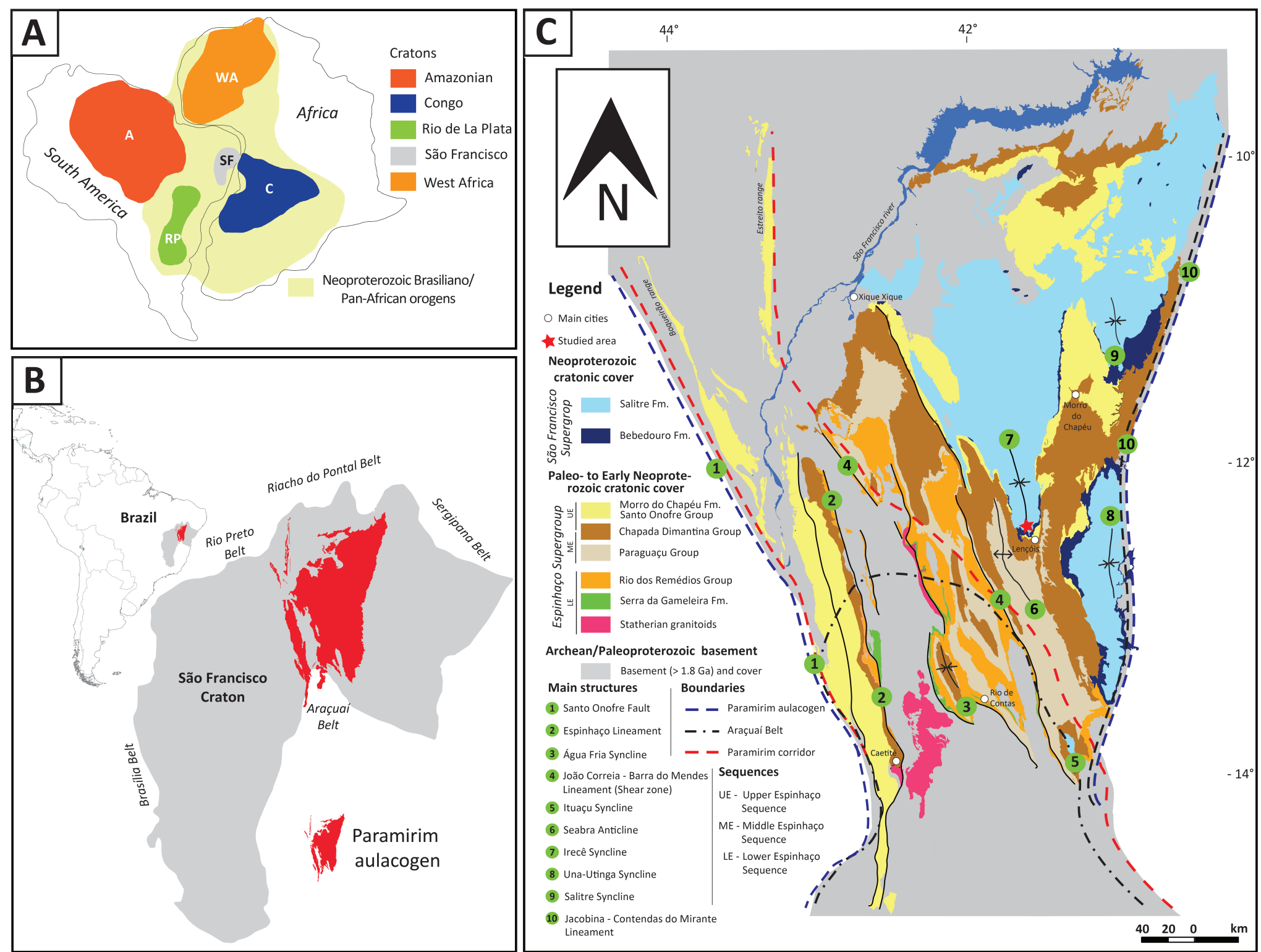

Figura 21. Geological context. (A) São Francisco Craton in the tectonic scenario of West Gondwana (modified from Alkmim et al., 2006). (B) Detail of the central portion of the São Francisco Craton and external mobile belts. (C) Simplified geological map of the Paramirin Aulacogen with localization of the studied area (star; modified from Souza et al., 2003; Guimarães et al., 2012). 


\subsection{Geological Setting}

The Irecê Basin units are distributed in the Irecê, Una-Utinga, Salitre and Ituaçu synclines in the Chapada Diamantina area domain of the São Francisco Craton (Fig. 21 C). It is divided into formations, the basal Bebedouro Fm. and overlying Salitre Fm. (e.g. Misi et al., 1998; and reference therein). These units occur above nonconformity with the cratonic basement (older than 1.8 Ga; Gavião Block; Barbosa and Sabaté, 2004) and an angular unconformity with the Paleoproterozoic to Neoproterozoic sedimentary cover (Espinhaço Supergroup). The Gavião Block is composed of Paleo- to Mesoarchean tonalite-trondhjemite-granodiorite (TTGs), Paleoarchean to Paleoproterozoic greenstone belt associations and rift-related basins (Peucat et al., 2002; Barbosa and Sabaté, 2004; Teles et al., 2015). Those units were partially reworked during the Transamazonian/Eburnean Cycle, from ca. 2.2 to $2.0 \mathrm{Ga}$ (Barbosa and Sabaté, 2004; Cruz et al., 2016). The cratonic cover is represented by sedimentary and rarely volcanoclastic rocks of Espinhaço Supergroup (Chemale Jr. et al. 1993; Alkmim and Martins-Neto, 2012), deposited from ca. 1.8 to 0.9 Ga in three megasequences, the Lower (ca. 1.8 - $1.6 \mathrm{Ga}$ ), Middle (ca. 1.55 - $1.38 \mathrm{Ga}$ ) and Upper Espinhaço sequences (ca. 1.2 - 0.9 Ga; Chemale Jr. et al., 2012; Guadagnin et al., 2015b).

The São Francisco Supergroup (hereafter SFS) is deposited in an intracratonic basin (Misi et al., 2011). In the Chapada Diamantina region it is comprised by Bebedouro and Salitre formations. Bebedouro Formation is composed of glacial-marine deposits (Karfunkel and Hoppe, 1988; Guimarães, 1996; Figueiredo et al., 2009). Youngest detrital zircon grains dated at $874 \pm 9$ Ma sets the maximum depositional age for the Bebedouro Formation, 
although several authors linked this unit to an Sturtian glacial epoch (e.g. Misi and Veizer, 1998; Figueiredo et al., 2009; ). Salitre Formation consists of marine platform carbonate deposits (Misi, 1979). It is in contact through a regional unconformity with the underlying stratigraphic unit (e.g. Guimarães, 1996). This formation has been subdivided into five informal units from bottom to top nominated by C, B, B1, A and A1 units (Fig. 22; Misi, 1979; Misi and Kyle, 1994). Unit C is formed by red argillaceous dolomite and stromatolites deposited in a restricted basin setting. This unit has been associated with cap dolomites, due to $\delta^{13} \mathrm{C}$ values close to $-6.04 \%$ and aragonite crystal fans (Torquato and Misi, 1977; Misi and Kyle, 1994; Figueiredo, 2008), which are considered from post-Sturtian successions (Hoffman and Schrag, 2002; Vieira et al., 2016; and references therein). Unit B comprises deep ramp and subtidal-to intertidal deposits (this study; Misi and Kyle, 1994) and unit B1 is formed predominantly by supratidal deposits (Misi and Kyle, 1994). Both units represent one typical shallowing-upward carbonate cycle (hundreds of meters thick), named Cycle I (Fig. 22; Misi, 1979). Units A and A1 comprise respectively deep platformal and subtidal deposits. These units represent a second shallowing-upward carbonate cycle (Cycle II; Fig. 22; Misi, 1979).

The SFS sequences were deformed into large-scale by two superimposed non-coaxial deformation patterns, (i) NNW-SSE trending open double doubly plunging folds, reverse faults and thrusts that are connected with deformation of Paramirim aulocogen, with tectonic vergence to the west; (ii) E-W strinking southern vergent tectonic structures with flexural to shear folds, thrusts and folding associated with to south-verging Riacho do Pontal and Rio Preto mobile belts (Chemale et al., 1993; Alkmim et al., 1996; Caxito and Uhlein, 2013). The deformation decreases towards south, so that the N-S trending Irecê, Una-Utinga, 
Salitre and Ituaçu Synclines display very well preserved sedimentary strata (Fig. 21 C). Structural and geophysical data suggest that the Irecê Basin was located on a faulted and tilted substrate with thickest sedimentary packages in the NW region, which it is characterized by the negative Bouguer anomaly (Duarte et al., 2012). However, this region is marked by the more intense deformation to that S-SE segment of Irecê Basin where the sedimentary rocks are just deformed mainly by open to gentle folded and preserve very well the primary structures.

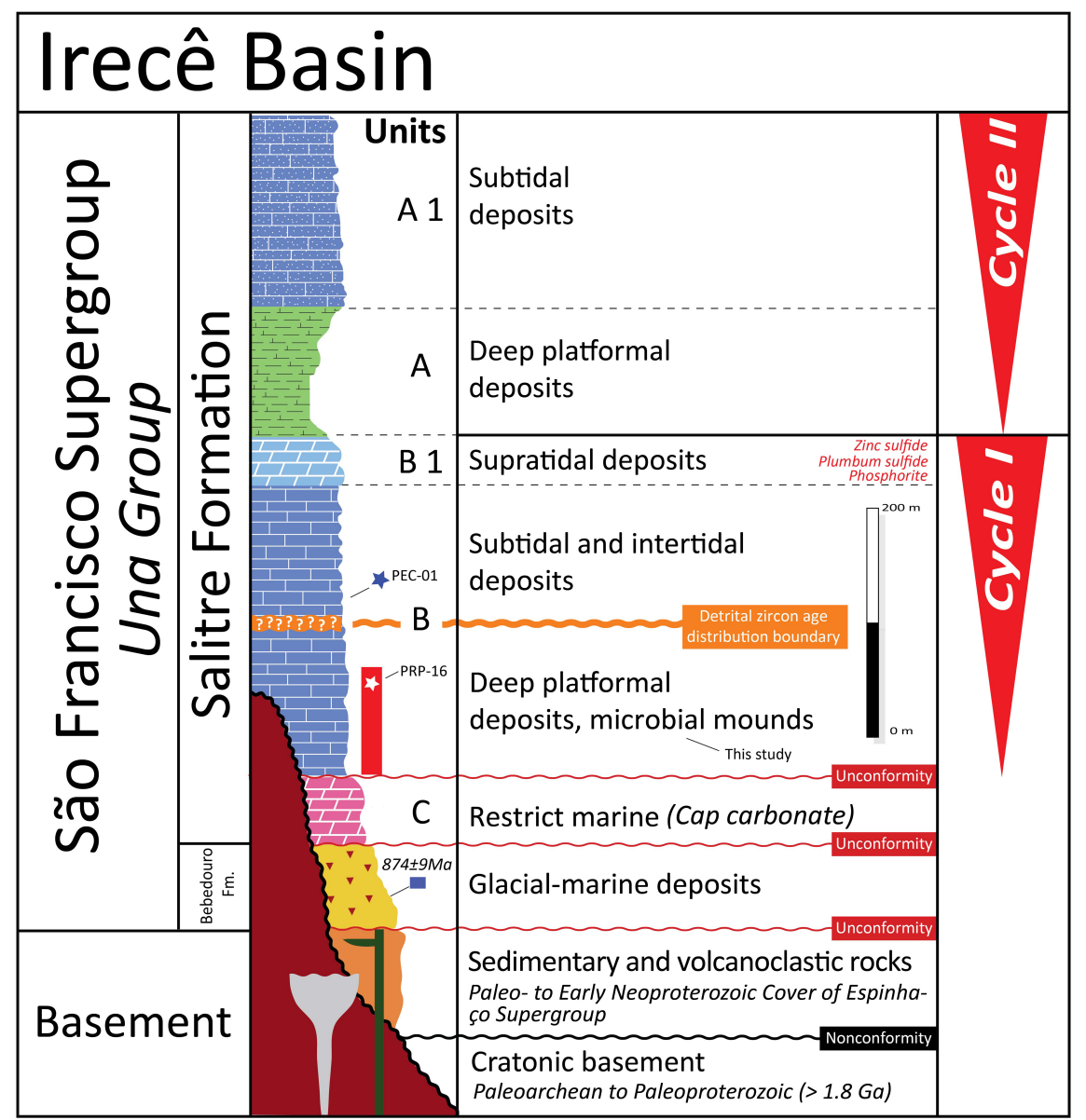

Figura 22. Stratigraphic column of the São Francisco Supergroup in Irecê Basin showing shallowing-upward carbonate cycles (hundreds of meters thick) identified in the Salitre Formation (Misi,1979; Misi and Kyle,1994). The red bar indicates location of the investigated section; star the analyzed sample location in this study; square indicates maximum depositional age for Bebedouro Formation (Figueiredo et al., 2009). 


\subsection{MATERIALS AND METHODS}

The methods applied include detailed facies analysis, elemental and stable isotope geochemistry and $\mathrm{U}-\mathrm{Pb}$ zircon dating. Facies analysis includes description of sedimentary facies in 1:20 scale vertical logs. Textural and mineral compositional analyses were made in thin sections and some samples were selected for mineral compositional analysis with X-ray diffractometry (XRD), Siemens D 5000 X-ray Diffractometer, at Universidade Federal do Rio Grande do Sul. The DECOMPXR ${ }^{\circledR}$ and NEWMOD ${ }^{\circledR}$ softwares (Reynolds, 1985) were used to improved characterization of clay components present in the samples. Chemical composition was determined by ICP-AES and the rare earth (REE) and refractory elements were determined by ICP-MS at Acme Analytical Laboratories. $\mathrm{Rb}$ and $\mathrm{Sr}$ contents were also measured using an X-Ray Fluorescence (Rigaku Primus II) at Universidade de Brasília.

For the stable isotopic analyses, $300 \mu \mathrm{g}$ of carbonate powders were obtained from 16 fresh samples at the Geochronology Laboratory of the Universidade de Brasília. The process was carried out by microdrilling of non-weathered and homogeneous samples. The isotopic ratios were obtained from reaction between $\mathrm{CO}_{2}$ extracted in a high vacuum chamber after reaction of powdered samples with $100 \% \mathrm{H}_{3} \mathrm{PO}_{4}$. The $\mathrm{C}$ and $\mathrm{O}$ isotopic compositions and $\mathrm{CO}_{2}$ values extracted were measured in a Delta Plus Advantage mass spectrometer. The results are expressed in $\delta$ notation and reported in parts per thousand (\%o), according to details relative to Vienna Pee Dee Belemnite standard (V-PDB notation; Verkouteren and Klinedinst, 2004) and calibrated against NBS-18 and NBS-19 standards, as mentioned in Alvarenga et al. (2014). To evaluate whether the isotope signature obtained is original or modified by postdepositional events, $\mathrm{Mn} / \mathrm{Sr}$ ratios were evaluated (Kaufman and Knoll, 1995; Brand and Veizer, 1980; Misi and Veizer, 1998).

5 NEW INSIGHTS OF GULF CARBONATE SEDIMENTATION OF THE IRECÊ BASIN 
Isotopic ratios were determined using Sensitive High Resolution Ion Microprobe (SHRIMP) analytical method at the Center of Geochronological Research (CPGeo) of Universidade de São Paulo and at the Western Australian University. U-Pb zircon analysis from one tuffite sample in the outcrop in log (Fig. 23; sample PRP-16) and a marlstone layer (sample PEC-16) were carried out at the Laboratory of High Resolution Geochronology of the Institute of Geosciences of University of São Paulo (GeoLab-IGc-USP) using SHRIMP I| e equipment. Tuffite and marlstone samples interlayered in the carbonate rocks and about $1 \mathrm{~kg}$ of rock was sampled from the bed for petrographic and geochronological studies.

U-Pb SHRIMP zircon preparation was done by hand picking zircon grains that were extracted by standard crushing, milling, then sieving $(0.150-0.063 \mathrm{~mm})$, wiffley table, and heavy liquid techniques (bromoform - density $=2.85$, and methylene iodine -density =3.32). Magnetic to non-magnetic fractions were separated using the Frantz ${ }^{\circledR}$ isodynamic separator and after removing ferromagnetic minerals with a hand magnet. Handpicked zircons were mounted in epoxy discs along with zircon standards (Temora 2), ground and polished, photographed in transmitted and reflected light, and imaged using scanning electron microscope (backscattered electrons - BSE) and cathode luminescence. Mounts were then cleaned and gold-coated in preparation for SHRIMP analysis.

Analytical procedures and data treatment follow the methods presented by Sato et al. (2014). Zircon grains were analyzed with a $2-3 \mathrm{nA}, 10 \mathrm{kV}$ primary 02 - beam focused to a 30 $\mu \mathrm{m}$ diameter spot with a beam density around 2.5-7 ๆA (dependent on brightness aperture). 
At mass resolution between 5000 and 5500 the $\mathrm{Pb}$, Th and $\mathrm{U}$ isotopes were resolved from all major interferences. Reduction of raw data and age calculation were carried out using Squid 1.06 and Isoplot-Ex (Ludwig, 2008). $U$ and Th concentrations were determined relative to those measured in the standard SL13, and Temora 2 was used to calculate the ${ }^{206} \mathrm{~Pb} /{ }^{238} \mathrm{U}$ age. Isoplot 3 software (Ludwig, 2003) was used to generate concordia diagrams, histograms and relative probability plots. For the concordia age calculations, frequency histograms and probability plots, only analyses that were within $100 \pm 10 \%$ of concordance were used.

\subsection{INTEGRATEd StUdies OF THe Rio Preto QuARRY SECTION}

\subsubsection{FACIES ANALYSIS}

A total of $40 \mathrm{~m}$ of rock succession are described. Seven facies are identified in the study section (Fig. 23) and their main characteristics are summarized in Table 2.

\section{MASSIVE MICROBIAL BOUNDSTONE (MM)}

Macroscopically, this facies consist of mud-supported limestone. It is show grey to dark grey colour and generally massive structure. Disseminated and/or small pockets of pyrite as well as dissolution seams are common. Internally, this facies shows peloidal and irregularly clotted microfabrics, and rare terrigenous grains, silt-size. Facies $\mathrm{Mm}$ have essentially tabular geometry and extents for hundreds of meters (Figs. 24 A and B) with thickness varies between few centimeters to up to $70 \mathrm{~cm}$. Lower contact is mainly abrupt but sometimes gradational with facies $\mathrm{He}, \mathrm{Fw}, \mathrm{Fl}$ or $\mathrm{Fl}_{(\mathrm{v})}$. Upper surfaces are irregular to sharp with facies $\mathrm{Fl}$, $\mathrm{Fw}, \mathrm{He}, \mathrm{Lm}$ and $\mathrm{Fl}_{(\mathrm{v})}$. Mud-supported fabric suggests a low energy deposition, to settle in calm waters (e.g., below storm wave base; Flügel, 2010). The presence of clotted and peloidal textures indicates which this facies was deposited in place due biologically induced 
and/or mediated precipitation from microbial process (Riding, 2000), consisting of microbialites (sensu Burne and Moore, 1987).

\section{Peloidal Wackestone- to packstone (WP)}

It corresponds to limestone composed of lime mud and peloids, terrigeneous and intraclasts of microbialites (ca. $15 \%$ until $60 \%$ of thin-section). It is grey colored and grain size vary from fine up to very-coarse grained sand. It is common the presence of dissolution features in WP facies and massive structure. Incipient parallel lamination and low-angle lamination are rare. Facies WP shows tabular geometry reaching ca. $1 \mathrm{~m}$ thick. The texture description suggests which facies WP were deposited in an environment with low- to moderate bottom hydrodynamics conditions (Flügel, 2010). The presence of low angle dipping laminae suggests the acting of oscillatory flow combined with unidirectional currents causing a lateral transport component, possibly because storm induced (Bádenas and Aurell, 2001). The occurrence of peloids sand-size grains suggests bottom reworking by current-induced from storm waves and that strengthen this hypothesis (Calvet and Tucker, 1988; Flügel, 2010). This implies a depositional setting below normal wave base and above storm wave base level. 


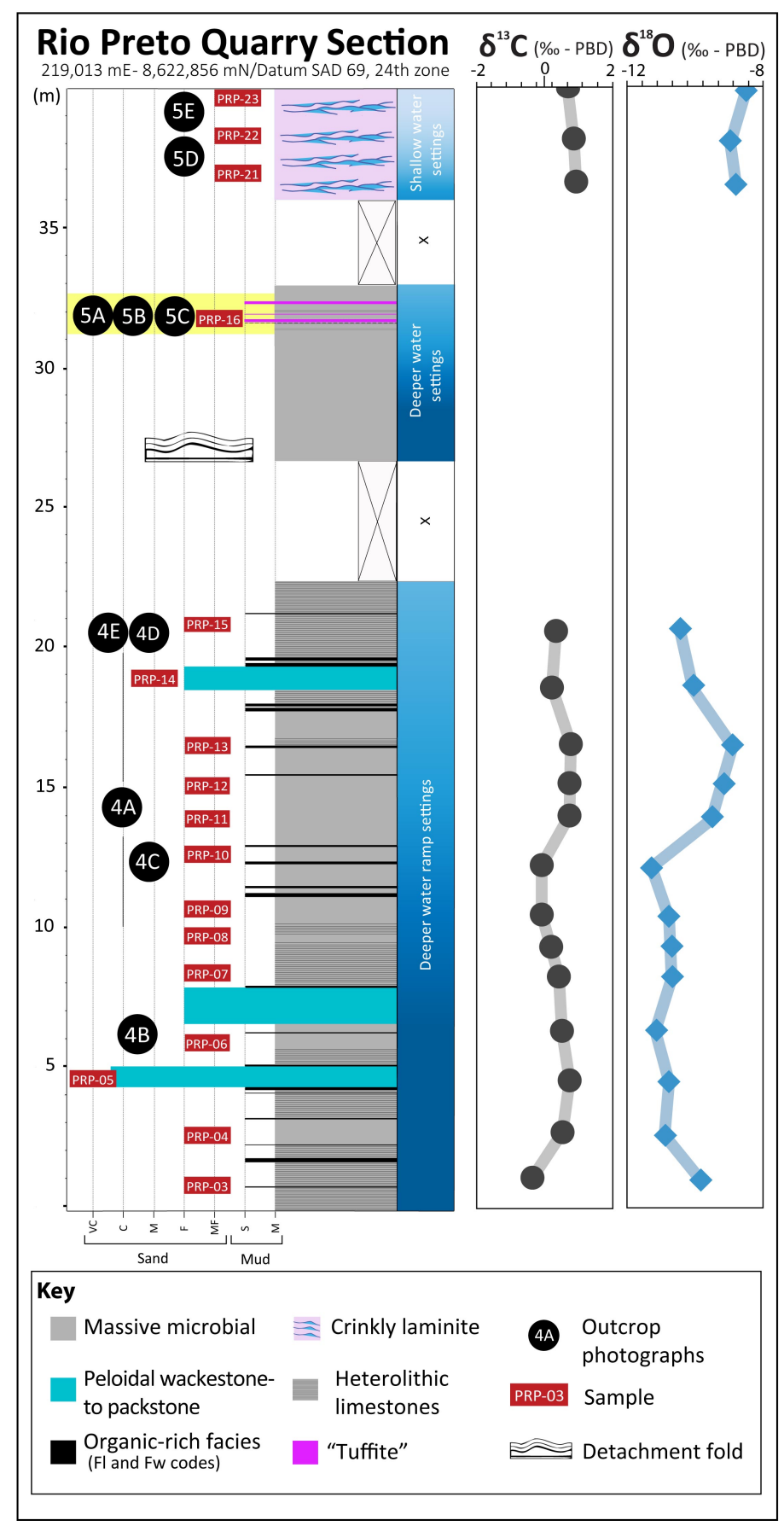

Figura 23. Facies, $C$ and $O$ profiles in the studied section. The tuffaceous interval what has geochronological analysis it's highlighted by a yellow stripe. The outcrop photographs mentioned in the article is also displayed. 
Table 2. Summary of the main characteristic features of the facies types identified in the Salitre Formation in this study area (Article 2)

\begin{tabular}{|c|c|c|c|c|c|c|c|c|}
\hline Facies (code) & Colour & $\begin{array}{l}\text { Major components (Petrology analysis and } \\
X^{*} \mathrm{D}^{*} \text { ). }\end{array}$ & Sedimentary structures & $\begin{array}{l}\text { Thickness } \\
(\mathrm{cm})\end{array}$ & Geomety and extent & Contacts & Processes & Interpretation \\
\hline \multicolumn{9}{|l|}{ Carbonate facies } \\
\hline $\begin{array}{l}\text { Massive microbial } \\
\text { boundstone (Mm) }\end{array}$ & Grey to dark grey & $\begin{array}{l}\text { Lime mud. Rare terrigenous grains, sitt-size. } \\
\text { Peloidal and irregularly clotted microfabrics }\end{array}$ & Massive & Up to 70 & $\begin{array}{l}\text { Tabular (Biostrome-type). Extends } \\
\text { by hundreds of meters }\end{array}$ & \begin{tabular}{|l|} 
Lower contact is abrupt, rarely gradational with \\
facies He, FI of Fw. Upper superficies is iriregular \\
to sharp with facies $\mathrm{Fl}, \mathrm{He}$ and $\mathrm{F} / \mathrm{F}$.
\end{tabular} & $\begin{array}{l}\text { Induced and/or mediated } \\
\text { precipitation from microbial } \\
\text { process }\end{array}$ & $\begin{array}{l}\text { Organosedimentary deposits. Low energy depositional } \\
\text { environment on below the storm wave base level. } \\
\text { Outer ramp }\end{array}$ \\
\hline Crinkly laminite (Lm) & Light grey-to grey & $\begin{array}{l}\text { Lime mud. Peloidal and clotted microfabrics. } \\
\text { Remains of microbial mats }\end{array}$ & | Irregular to wrinkled lamination & ca. 400 & $\begin{array}{l}\text { Tabular (Biostrome-type). Bodies } \\
\text { extends hundreds of meters }\end{array}$ & - & Trapping and binding & $\begin{array}{l}\text { Organosedimentary deposits. Low- to moderada } \\
\text { energy deposition. Below normal wave base and above } \\
\text { storm wave base level. Middle ramp }\end{array}$ \\
\hline $\begin{array}{l}\text { Peloidal wackestone- to } \\
\text { packstone (WP) }\end{array}$ & Grey & $\begin{array}{l}\text { Lime mud and peloids, terrigeneous and } \\
\text { intraclasts of microbialites (ca. 15\% until } \\
60 \% \text { of thin-section) }\end{array}$ & \begin{tabular}{|l} 
Massive. Incipient parallel \\
lamination and low-angle \\
lamination
\end{tabular} & ca. 100 & $\begin{array}{l}\text { Tabular. Extends by hundreds of } \\
\text { meters }\end{array}$ & 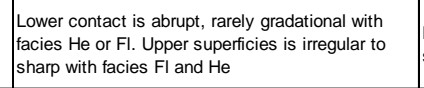 & $\begin{array}{l}\text { Bottom reworking by currents, } \\
\text { storm induced }\end{array}$ & $\begin{array}{l}\text { Low-to moderada energy deposition. Below normal } \\
\text { wave base and above storm wave base level. Middle } \\
\text { ramp }\end{array}$ \\
\hline \multicolumn{9}{|l|}{ Carbonaceous-rich facies } \\
\hline \begin{tabular}{|l} 
Laminated siliciclastic \\
mudstone (FI)
\end{tabular} & Black - Dark grey & $\begin{array}{l}\text { Quartz and K-feldspar, silt-size. Pyrite and } \\
\text { carbonaceous material }\end{array}$ & Horizontal lamination & $5-15$ & $\begin{array}{l}\text { Tabular. A few amalgamated. } \\
\text { Packages extends hundreds of } \\
\text { meters }\end{array}$ & $\begin{array}{l}\text { Lower boundary is sharp or irregular with facies } \\
\text { Mm, WP or He. Upper boundary is sharp or } \\
\text { gradatational with facies He }\end{array}$ & Suspension & $\begin{array}{l}\text { Low energy depositional enuironment. Below storm } \\
\text { wave base level. Water oxygen impoverishment. } \\
\text { Basin }\end{array}$ \\
\hline $\begin{array}{l}\text { Wayy carbonaceous } \\
\text { calcitic-siliciclastic } \\
\text { mudstone (Fw) }\end{array}$ & Black & Carbonaceous material. Quartz, silt-size & Massive. Wawy bedding & $5-15$ & $\begin{array}{l}\text { Tabular. A few amalgamated. } \\
\text { Packages extends hundreds of } \\
\text { meters }\end{array}$ & $\begin{array}{l}\text { Lower boundary is sharp or irregular with facies } \\
\text { Mm, WP or He. Upper boundary is sharp or } \\
\text { gradatational with facies He }\end{array}$ & $\begin{array}{l}\text { Suspension. Occasional wave } \\
\text { reworking }\end{array}$ & $\begin{array}{l}\text { Low energy depositional enuironment. Below storm } \\
\text { wave base level. Water oxygen impoverishment. } \\
\text { Basin }\end{array}$ \\
\hline \multicolumn{9}{|l|}{ Heterolithic facies } \\
\hline $\begin{array}{l}\begin{array}{l}\text { Heterolithic limestones } \\
(\mathrm{He})\end{array} \\
\end{array}$ & \begin{tabular}{|l} 
Light grey and black \\
color
\end{tabular} & $\begin{array}{l}\text { Lime mud and siliciclastic/organic-rich } \\
\text { material }\end{array}$ & $\begin{array}{l}\text { Wawy and lenticular bedding. } \\
\text { Planar or faint undulating beds }\end{array}$ & Up to 100 & $\begin{array}{l}\text { Tabular. Bodies extends hundreds of } \\
\text { meters }\end{array}$ & $\begin{array}{l}\text { Lower boundary is gradational with facies } \mathrm{Fl} \text { or } \mathrm{FW} \\
\text { Upper normaly is irregular or sharp with facies } \mathrm{Mm} \\
\text { and WP }\end{array}$ & $\begin{array}{l}\text { Alternation of suspension } \\
\text { processes and oscillatory } \\
\text { flows }\end{array}$ & $\begin{array}{l}\text { Low energy depositional environment. Below storm } \\
\text { wave base level. Occasional storm wave action. Outer } \\
\text { ramp }\end{array}$ \\
\hline \multicolumn{9}{|c|}{ Mixture pyroclastic and epiclastic facies } \\
\hline $\begin{array}{l}\text { Tuffaceous siltstone- to } \\
\text { mudstone }\left(F_{(v)}\right)\end{array}$ & ochre & Quartz. Sanidine* and Smectite ${ }^{\star}$ & Horizontal lamination & $5-10$ & Tabular & $\begin{array}{l}\text { Lower and upper contacts are irregular to sharp } \\
\text { with facies } \mathrm{Mm}\end{array}$ & Suspension & $\begin{array}{l}\text { Represents low energy sedimentation with volcanic } \\
\text { material addition during deposition. Outer ramp }\end{array}$ \\
\hline
\end{tabular}




\section{LAMINATED SILICICLASTIC MUDSTONE (FL)}

Facies $\mathrm{Fl}$ consist of laminated claystone and siltstone intercalated with millimeter- to centimeter-thick of very fine-grained sandstones and thin carbonaceous-rich material. It is a dark coloured facies with horizontal lamination (Fig. 24 C).This facies is laterally extensive in the order of hundreds of meters. It is interpreted as low energy suspension sedimentation products due settling of marine pelagic from the water column (Flügel, 2010). Lack of wavy influenced structures suggests deposition well below storm wave base level (Calvet and Tucker, 1988).

\section{WAVY CARBONACEOUS CALCITIC-SILICICLASTIC MUDSTONE (FW)}

This facies comprises an organic-rich mudstone. It is massive, with faint/unclear lamination, rarely with wavy bedding (symmetric wavy ripples) on top of some beds. Facies Fw is black in colour and have finely disseminated pyrite. Amalgamated small-scale wavy ripples from 4 to $6 \mathrm{~cm}$ wavelength occur within up to $15 \mathrm{~cm}$ thick tabular beds (Fig. $24 \mathrm{~B}$ ). Facies Fw is laterally extensive in the order of hundreds of meters. The presence of the symmetrical ripples indicates oscillatory flows action and suggests reworking of the bottom sediment by storm wavy (Bádenas and Aurell, 2001).

\section{Heterolithic limestones (He)}

Facies He consists in levels of rhythmical intercalations of clearly segregated siliciclastic/organic-rich material and lime mud. Lime mud dominated beds have 2 to $5 \mathrm{~cm}$ thickness and light grey color. These levels exhibit wavy bedding (Figs. 24 D and E). Siliciclastic mud/organic-rich material dominated beds are consisted of silt-size and minor 
proportions of lime mud, with few centimeters thickness and black color. Some symmetrical wavy ripples with 3 to $6 \mathrm{~cm}$ wavelength, occurs mainly in the basal portion of measured vertical-log. Facies He have tabular geometry with significant lateral continuity and up to 1 meter thick. This facies is interpreted to be deposited by the alternation of suspension processes and oscillatory flows (Calvet and Tucker, 1988; Bádenas and Aurell, 2001; Flügel, 2010).

\section{TUfFACEOUS SILTSTONE- TO MUDSTONE $\left(\mathrm{FL}_{(\mathrm{V})}\right)$}

Tuffaceous siltstone- to mudstone facies $\left(\mathrm{FI}_{(\mathrm{v})}\right)$, herein also referred to as "tuffite" (sensu Le Maitre, 2002), are composed of a mixture of both pyroclasts and epiclasts, and the amount of pyroclasts may vary from $75 \%$ to $25 \%$. It is light brown and horizontal laminated (Figs. $25 \mathrm{~A}$ and B), composed of silt-size grains ( 15\%) immersed in a very-fine grained matrix. Pyroclasts are consisted of fragments of subhedral to euhedral crystals of Quartz and KFeldspar. Mineral phases also identified with XRD are Smectite, Clorite, Calcite, Sanidine and Ankerite. Facies $\mathrm{Fl}_{(\mathrm{v})}$ have tabular bed geometry, up to $10 \mathrm{~cm}$ thick. Lower and upper contacts are irregular to sharp with facies $\mathrm{Mm}$. Observed horizontal lamination and texture are particularly indicative of deposition in low energy environment from suspension process (Simons et al., 1965). Crystals clast strongly indicates an volcanic material addition during deposition (Schmid, 1981). 


\section{CRINKLY LAMINITE (LM)}

Facies Lm consists of limestone with fine- to very fine-grained carbonates levels arranged in irregular to wrinkled lamination with millimeter scale (Figs. $25 \mathrm{D}$ and E). This facies is light grey- to grey colored with brown/ocher laminae and shows abundance of dissolution features. The texture is characterized by peloidal and clotted features. Lm facies commonly presents tabular bodies arranged into up to $4 \mathrm{~m}$ thick (Fig. $25 \mathrm{D}$ ). This facies is interpreted as resulting from organic production that resulted in the trapping and binding process carried out through colonies of cyanobacteria. Microfabrics and irregular- to wrinkled laminae are highly suggestive of microbially influenced sedimentation and serve as clear confirmation of organosedimentary deposits (Burne and Moore, 1987; Terra et al., 2010; Grotzinger and AlRawahi, 2014). Their tabular geometry indicates biostrome-type build-ups (Walter, 1976). 

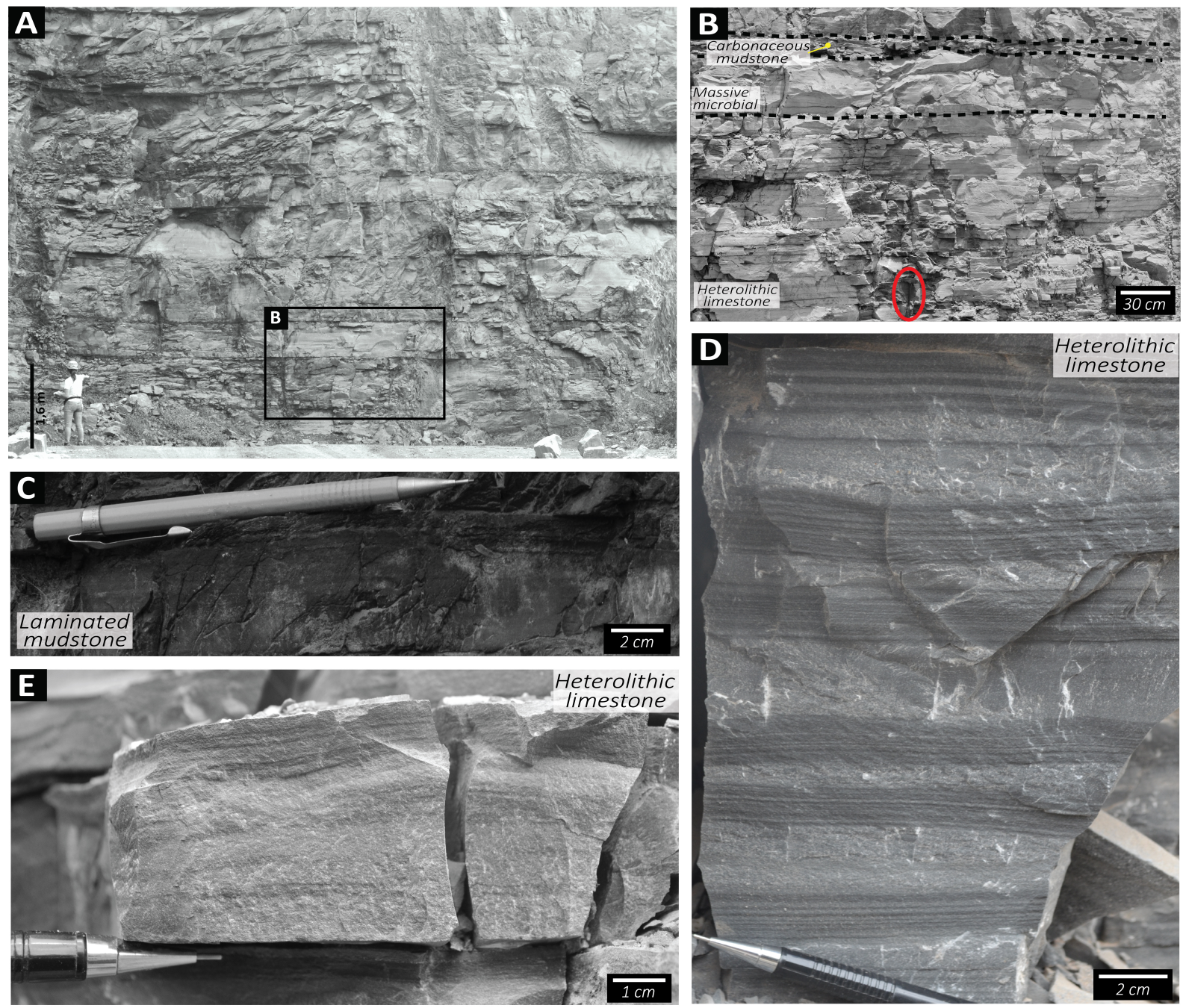

Figura 24. Characteristic examples of facies described in the Salitre Formation in the Rio Preto Quarry. (A) General view showing tabular geometry and extensive of the bodies. $(B)$ Inset of $(A)$, show the contacts irregular-to sharp of the different facies. (C) Horizontally laminated claystone- to siltstone, in the facies FI. (D) and (E) Levels exhibit wavy bedding (facies He). 

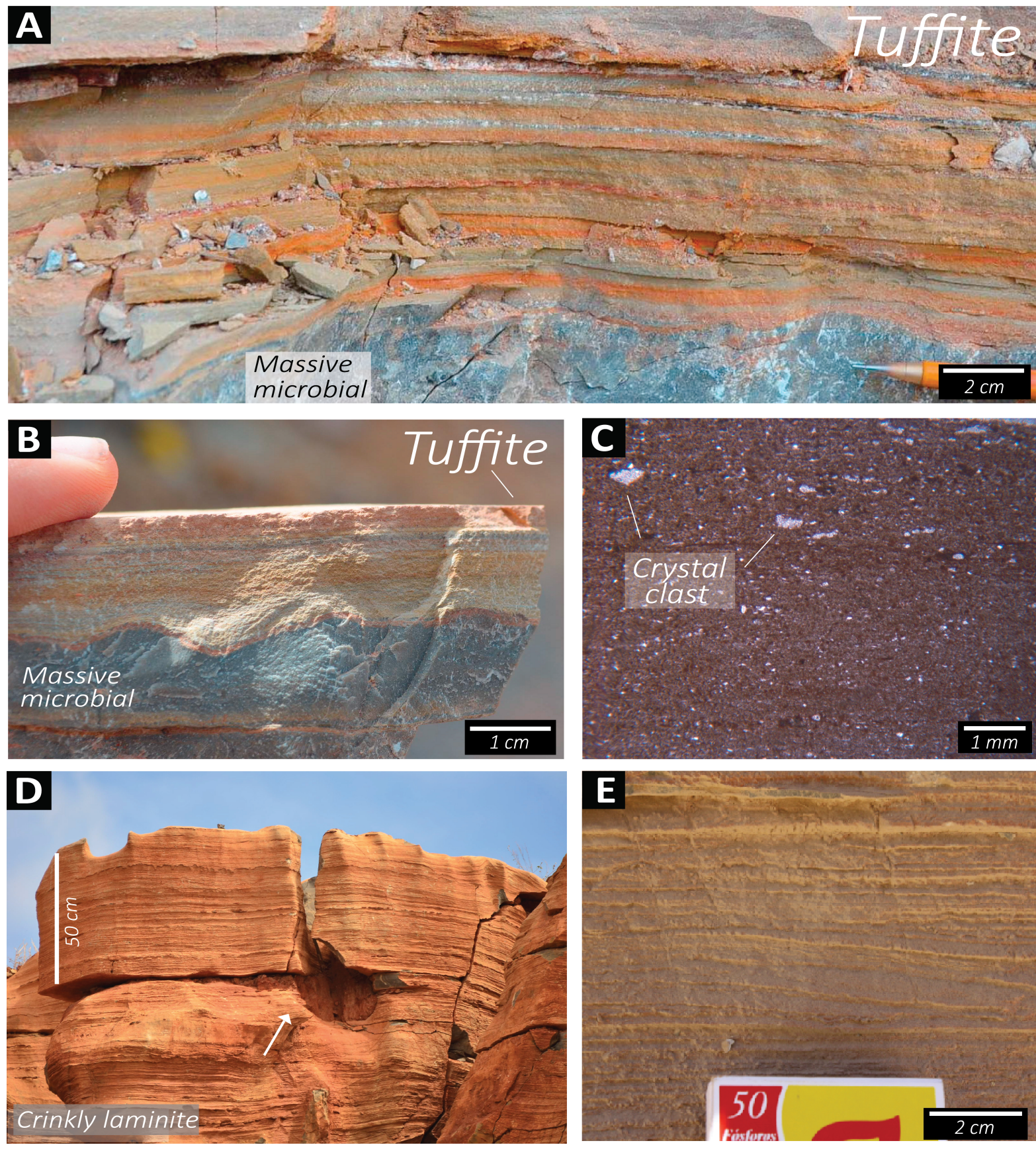

Figura 25. Characteristic examples of facies described in the Salitre Formation in the Rio Preto Quarry (continuation). (A) and (B) Horizontally laminated in the tuffaceous siltstone- to mudstone, facies FI(v). (C) Main petrographic characteristics of the facies $F I(v)$ are abundant crystals clast immersed in a very-fine-grained matrix. (D) Crinkly laminite facies highlight to occurrence the karst topography (arrow). (E) Fine- to very finegrained carbonate levels arranged in irregular to wrinkled lamination with $\mathrm{mm}$-scale, facies $\mathrm{Lm}$. 


\subsubsection{AND O STABLE ISOTOPE RESULTS}

Samples analyzed for stable isotopes were collected at the base and top of measured vertical-log (Fig. 23). Values of $\delta^{13} \mathrm{C}$ and $\delta^{18} \mathrm{O}$ for each sample are shown in the Table 3. Uncertainties of isotopes measurements were $0.05 \%$ for $\delta^{13} \mathrm{C}$, and $0.10 \%$ o for $\delta^{18} \mathrm{O}$. Additionally, $\mathrm{Mn}$ and $\mathrm{Sr}(\mathrm{ppm})$, as well as specific ratios used to evaluate samples preservation are also presented in Table 3. Element composition, detection limits, samples weights and QA/QC data are reported in the Supplementary material 1. Lower interval of measured vertical-log has $\delta^{13} \mathrm{C}$ values ranging between -1.12 and $-0.28 \%$ and $\delta^{18} \mathrm{O}$ values from -12.22 to $-9.45 \%$ o (Fig. 23). Sr concentration in this interval is between 197 and 300 ppm; Mn contents is between 465 and $852 \mathrm{ppm}$; and Mn/Sr ratios ranging from 2.21 to 3.21 . Upper interval has $\delta^{13} \mathrm{C}$ values between -0.37 and $-0.22 \%$ and $\delta^{18} \mathrm{O}$ values from -9.49 to 9.12\%o (Fig. 23). Sr concentration in this interval is higher, reaching $600 \mathrm{ppm}$; Mn contents are lower, down to $155 \mathrm{ppm}$; and $\mathrm{Mn} / \mathrm{Sr}$ ratios ranging from 1.74 and 0.39 . 
Table 3. $\mathrm{C}$ and $\mathrm{O}$ isotopes values, $\mathrm{Mn}$ and $\mathrm{Sr}$ contents (ppm) and geochemical ratios obtained on analyzed carbonates. Error $\delta^{13} \mathrm{C}=0,05 \%$; $\delta^{18} \mathrm{O}=0,10 \%$.

\begin{tabular}{|l|l|l|l|l|l|}
\hline Sample & $\boldsymbol{\delta}^{13} \mathbf{C} \%$ & $\boldsymbol{\delta}^{18} \mathbf{O} \%$ & $\mathbf{M n}(\mathbf{p p m})$ & $\mathrm{Sr}(\mathbf{p p m})$ & $\mathbf{M n} / \mathrm{Sr}$ \\
\hline PRP-23 & -0.37 & -9.12 & 232.00 & 600 & 0.39 \\
\hline PRP-22 & -0.24 & -9.49 & 697.00 & 400 & 1.74 \\
\hline PRP-21 & -0.22 & -9.34 & 155.00 & 300 & 0.52 \\
\hline PRP-15 & -1.12 & -10.88 & 852.00 & 209 & 4.08 \\
\hline PRP-14 & -0.99 & -10.22 & 465.00 & 348.7 & 1.33 \\
\hline PRP-13 & -0.72 & -10.60 & 620.00 & 197.2 & 3.14 \\
\hline PRP-12 & -0.39 & -10.68 & 542.00 & 243.1 & 2.23 \\
\hline PRP-11 & -0.69 & -10.29 & 542.00 & 222.3 & 2.44 \\
\hline PRP-10 & -0.28 & -9.43 & 465.00 & 217.7 & 2.14 \\
\hline PRP-09 & -0.35 & -9.89 & 852.00 & 230.9 & 3.69 \\
\hline PRP-08 & -0.93 & -11.22 & 465.00 & 265.6 & 1.75 \\
\hline PRP-07 & -0.97 & -10.86 & 465.00 & 210.2 & 2.21 \\
\hline PRP-06 & -0.54 & -10.78 & 542.00 & 256.6 & 2.11 \\
\hline PRP-05 & -0.50 & -11.17 & 620.00 & 212.8 & 2.91 \\
\hline PRP-04 & -0.33 & -10.86 & 697.00 & 267.6 & 2.60 \\
\hline PRP-03 & -0.47 & -10.95 & 852.00 & 294.5 & 2.89 \\
\hline
\end{tabular}




\subsubsection{GEOCHEMISTRY OF THE TUFFITE LAYER}

Tuffite facies (sample PRP-16) is identified at the 31.45 meter of measured vertical-log (Fig. 23) and their stratigraphic position is shown in Figure 22. Facies description is presented in Section 6.4.1 and geochemical composition is presented in Supplementary material 1. Mixed pyroclastic-epiclastic deposits have complex chemical compositions; they reflect a mixture of the different components subjected to various modification processes. Thus, it is very difficult to determine the amount each source rock contributed to these rock types and gather provenance information (McLennan et al., 1993), whereas several authors describe the chemical compositions of such deposits (e.g., Le Bas et al., 1986; Roser and Korsch, 1988; McLennan et al., 1993; Wedepohl, 1995; Alessandretti et al., 2013; Guadagnin et al., 2015a; and others). Trace and rare-earth elements are best suited for petrologic analysis of such deposits because they are less mobile than major elements and reflect original composition mixed in varying proportions (McLennan et al., 1993). Comparison of tuffite composition with composition of the Upper Continental Crust (Taylor and McLennan, 1985) and Proterozoic volcanics and sedimentary rocks (Condie, 1993) help to constrain tuffite sources.

Only trace and rare-earth elements (REE) were used. High-field strength elements totaled 185.6 ppm, mostly represented by $\mathrm{Zr}$, which has $151.6 \mathrm{ppm}$ in analyzed sample. Large-ion lithophile elements totaled 1,648.4 ppm, mostly represented by Ba which has 1,398 ppm (Supplementary material 2). Total REE content is 161.78 ppm; Light REE (LREE) are enriched relative to the Heavy REE (HREE). LREE/HREE ratio is 10.93; La/Yb ratio is 19.94; and La/Yb(n) ratio is 13.48 . $\mathrm{La} / \mathrm{Sm}(\mathrm{n})$ ratio is 3.81 and $\mathrm{Gd} / \mathrm{Yb}(\mathrm{n})$ ratio is $2.22 . \mathrm{Gd}(\mathrm{n})$ value is 15.49 . Eu anomaly (Eu/Eu*) is 0.61 (Supplementary material 3). Compared to the composition of the 
UCC (Taylor and McLennan, 1985), Tuffite composition is enriched in $\mathrm{Ba}, \mathrm{Rb}, \mathrm{Sc}, \mathrm{V}, \mathrm{Th}$ and $\mathrm{U}$, and depleted in $\mathrm{Co}, \mathrm{Zr}, \mathrm{Hf}, \mathrm{Nb}$ and Ta, whereas highly depleted in Sr (Fig. $26 \mathrm{~A}$ ). This pattern is partially similar to felsic volcanic rocks, volcaniclastic wackestones and shales (Fig. $26 \mathrm{~A}$; Condie, 1993). Tuffite LREE are enriched relative to the UCC, whereas HREE are depleted, except for Gd (Fig. 26 A). This pattern is quite similar to Cryogenian Alkaline Syenites REE composition (Rosa et al., 2005). Tuffite total REE composition is enriched relative to the UCC, as well as LREE/HREE, La/Yb, (La/Yb)n and $(\mathrm{Gd} / \mathrm{Yb}) \mathrm{n}$ ratios and $\mathrm{Gdn}$; Eu anomaly and (La/Sm)n ratio are slightly depleted relative to the UCC (Fig. $26 \mathrm{~A}$ ).

Depletion of $\mathrm{Zr}, \mathrm{Hf}, \mathrm{Nb}$ and $\mathrm{Ta}$ in Tuffite relative to the UCC is suggestive of derivation from Andesite sources (Fig. 26 A; Supplementary material 2; Condie, 1993). In the classification of igneous rocks scheme based on Winchester and Floyd (1977), from $\mathrm{Zr} / \mathrm{TiO} 2$ and $\mathrm{Nb} / \mathrm{Y}$ ratios, samples plot in the Trachy-Andesite field (Fig. 26 B), partially overlapping the composition of Paleozoic subaqueous fine-grained volcaniclastic rocks ("Tonsteins"; Formoso et al., 1999; Dani et al., 2011) and close to the composition of the Upper Continental Crust (UCC; Taylor and McLennan, 1985). Tuffite composition plot in Granite of volcanic arc field in the $\mathrm{Yb}$ versus Ta diagram (Pearce et al., 1984) close to Andesite and volcaniclastic wackestone compositions (Fig. 26 C; Condie, 1993). Chondrite normalized REE show enrichment of LREE relative to HREE, negative Eu anomalies and flat HREE pattern, which is similar to volcaniclastic wackestones (Fig. 26 D; Condie, 1993; Boynton, 1984). 

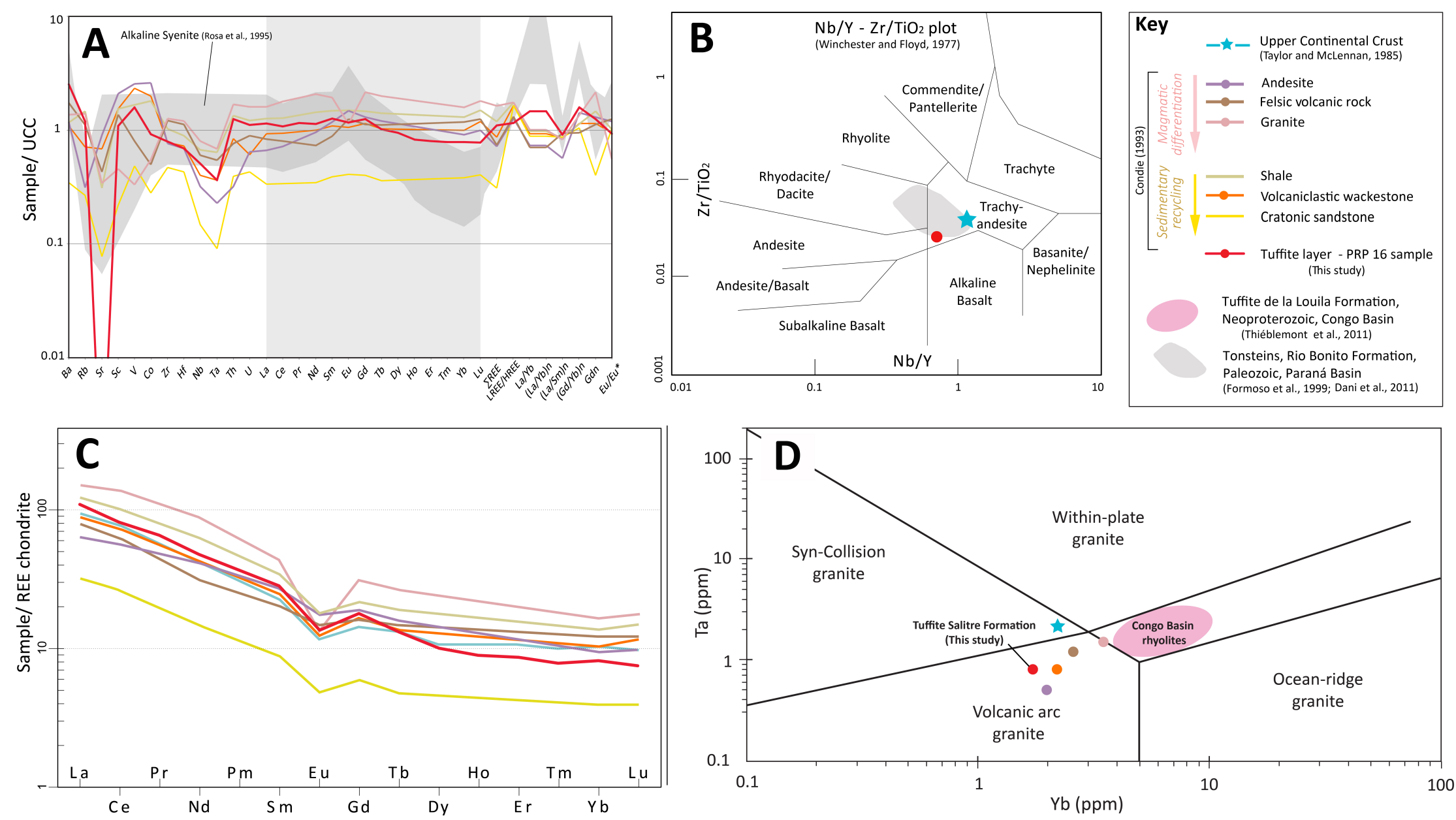

Figura 26. Geochemical plots for the tuffite layer. A - A volcanic rocks discrimination diagram (Winchester and Floyd, 1977) with tuffite sample plotted as signature of the magma with a trachyandesitic composition. Taylor and McLennan (1985) and Formoso et al. (1999) show the data used for the comparison. B - Data plotted in the specific multielement diagram normalized to chondrite (Thompson, 1982). C - Spider diagram of the REE normalized to the chondrite (Boynton, 1984) which indicates the tuffite layer and reference values from different rocks of the Proterozoic obtained in Condie (1993) and Taylor and McLennan (1985). 


\subsubsection{GEOCHRONOLOGICAL DATA}

In order to determine the source and maximum depositional age of the carbonate package of the Unit B, U-Pb zircons analyses of the tuffite layer (sample PRP-16) and marlstone layer (sample PEC-01) have carried out. The PEC-01 is exposed in the uppermost section of the carbonate platform, approximately 150 meters above the PRP-16 tuffite (Fig. 23). The U-Pb analytical data are presented in the Table 4.140 zircon grains within $100 \pm 10 \%$ of concordance of sample PRP-16 have been analyzed. Zircon grains from the sample PRP-16 are typically elongated and short prismatic crystals with mean diameter from 50 to $200 \mu \mathrm{m}$ (Fig. 27).

Others grains were rounded to sub-rounded, and few consisted of broken crystal fragments. In general, the grains are zoned in the cathodoluminescence images, with only some grains homogeneous. The youngest zircon grain of the tuffite (PRP-16) dates in the Cryogenian period (850-635 Ma) and presents a concordia age of $669 \pm 14 \mathrm{Ma}(1 \sigma)$, which is interpreted as a maximum depositional age for this stratigraphic interval or as the depositional age as in the case this grain is a juvenile volcanic zircon grain. The rest of zircon populations can be grouped of three major groups: Archean (3388 Ma), Neoarchean (2565 Ma) and Rhyacian (2075 Ma). Few zircon grains (6) formed between $1.8 \mathrm{Ga}$ and $1.3 \mathrm{Ga}$ (Fig. $28 \mathrm{~A}$ ). The overlying marlstone layer (sample PEC-1) contains a distinct zircon age distribution pattern with main peak at $3292 \mathrm{Ma}, 2630 \mathrm{Ma}, 2102 \mathrm{Ma}$ as the main zircon population (Fig. $28 \mathrm{~B}$ ). There also a significant contribution of zircons formed during the Statherian (peak at 1755Ma) and Calymnian (at $1500 \mathrm{Ma}$ ). 


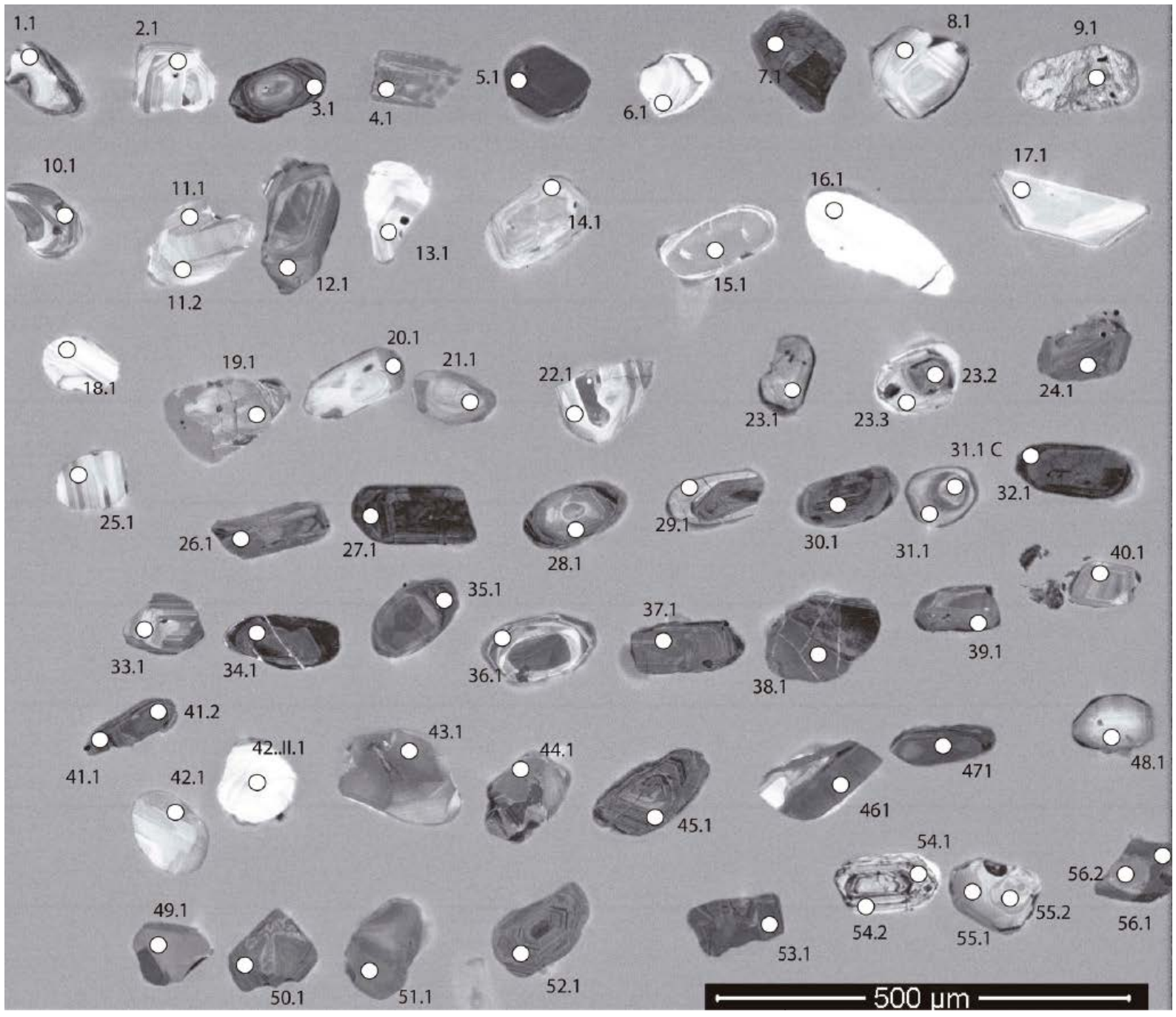

Figura 27. Cathodoluminescence images of the zircon grains from the tuffite sample. 
Table 4. Summary of SHRIMP U-Pb zircon data for samples PRP-16 (Rio Preto Quarry) and PEC-01 (Pecuária Cave).

\begin{tabular}{|c|c|c|c|c|c|c|c|c|c|c|c|c|c|c|c|c|}
\hline Spot Name & $\begin{array}{l}\text { (1) } \% \\
{ }^{206} \mathrm{~Pb}_{\mathrm{c}} \\
\end{array}$ & $\begin{array}{c}\mathrm{ppm} \\
\mathrm{U}\end{array}$ & $\begin{array}{c}\mathrm{ppm} \\
\mathrm{Th}\end{array}$ & $\begin{array}{l}{ }^{232} \mathrm{Th} \\
\mathrm{I}^{238} \mathrm{U} \\
\end{array}$ & $\begin{array}{c}(1) \\
{ }^{206} \mathrm{~Pb} \\
{ }^{238} \mathrm{U} \\
\text { Age }\end{array}$ & $\begin{array}{l}1 \mathrm{~s} \\
\text { err }\end{array}$ & $\begin{array}{c}(1) \\
{ }^{207} \mathrm{~Pb} \\
\rho^{206} \mathrm{~Pb} \\
\text { Age }\end{array}$ & $\begin{array}{l}1 \mathrm{~s} \\
\text { err }\end{array}$ & $\begin{array}{c}\% \\
\text { Dis- } \\
\text { cor- } \\
\text { dant } \\
\end{array}$ & $\begin{array}{c}(1){ }^{207} \mathrm{~Pb}^{*} \\
{ }^{206} \mathrm{~Pb}^{*}\end{array}$ & $\begin{array}{l}\% \\
\mathrm{err}\end{array}$ & $\begin{array}{c}\text { (1) }{ }^{207} \mathrm{~Pb}^{\star} \\
\mathrm{I}^{235} \mathrm{U}\end{array}$ & $\begin{array}{l}\% \\
\text { err }\end{array}$ & $\begin{array}{c}(1){ }^{206} \mathrm{~Pb}^{*} \\
{ }^{238} \mathrm{U} \\
\end{array}$ & $\begin{array}{l}\% \\
\text { err }\end{array}$ & $\begin{array}{l}\text { err } \\
\text { corr }\end{array}$ \\
\hline \multicolumn{17}{|l|}{ Sample PRP-16 } \\
\hline PRP-16-B-31.1 & 0.90 & 272 & 68 & 0.26 & 664.3 & 21 & 645 & 116 & -3 & .0612 & 5.4 & 0.915 & 6.3 & 0.109 & 3.3 & .52 \\
\hline PRP-16B-31.C & 0.44 & 315 & 72 & 0.24 & 675.4 & 20 & 655 & 50 & -3 & .0614 & 2.3 & 0.936 & 3.9 & 0.110 & 3.1 & .79 \\
\hline prp-16--24A.1 & 0.133 & 195 & 98 & 0.517 & 2647 & 37 & 2652 & 11 & 0 & 1799 & 0.66 & 12.594 & 1.8 & 0.508 & 1.7 & 0.93 \\
\hline prp-16--24A.2 & 0.300 & 89 & 47 & 0.546 & 2706 & 48 & 2449 & 54 & -10 & .1594 & 3.16 & 11.463 & 3.8 & 0.522 & 2.2 & 0.57 \\
\hline prp-16--24A.3 & 1.267 & 123 & 178 & 1.499 & 1892 & 32 & 2122 & 37 & 11 & .1318 & 2.12 & 6.201 & 2.9 & 0.341 & 1.9 & 0.68 \\
\hline prp-16--24A.4 & 0.283 & 132 & 57 & 0.445 & 2646 & 71 & 2679 & 14 & 1 & .1828 & 0.83 & 12.793 & 3.4 & 0.507 & 3.3 & 0.97 \\
\hline prp-16--24A.5 & 0.030 & 212 & 106 & 0.515 & 2623 & 35 & 2627 & 10 & 0 & .1772 & 0.59 & 12.267 & 1.7 & 0.502 & 1.6 & 0.94 \\
\hline prp-16--24A.6 & 1.387 & 143 & 127 & 0.917 & 1753 & 31 & 2100 & 37 & 17 & .1302 & 2.09 & 5.607 & 2.9 & 0.312 & 2.0 & 0.70 \\
\hline prp-16--24A.7 & 0.061 & 204 & 120 & 0.609 & 2632 & 35 & 2550 & 10 & -3 & .1692 & 0.61 & 11.765 & 1.7 & 0.504 & 1.6 & 0.94 \\
\hline prp-16--24A.8 & 0.083 & 164 & 94 & 0.594 & 2921 & 40 & 2880 & 10 & -1 & .2067 & 0.62 & 16.341 & 1.8 & 0.573 & 1.7 & 0.94 \\
\hline prp-16--24A.10 & 0.078 & 122 & 165 & 1.402 & 2175 & 35 & 2091 & 19 & -4 & .1295 & 1.07 & 7.166 & 2.2 & 0.401 & 1.9 & 0.87 \\
\hline prp-16--24A.13 & 0.411 & 284 & 283 & 1.030 & 1989 & 41 & 2102 & 16 & 5 & .1303 & 0.88 & 6.494 & 2.5 & 0.362 & 2.4 & 0.94 \\
\hline prp-16--24A.14 & 0.400 & 116 & 42 & 0.373 & 2682 & 43 & 2642 & 34 & -2 & .1788 & 2.02 & 12.716 & 2.8 & 0.516 & 2.0 & 0.70 \\
\hline |prp-16--24.B-1 & 0.000 & 77 & 36 & 0.481 & 2968 & 50 & 3171 & 12 & 6 & .2479 & 0.76 & 19.988 & 2.2 & 0.585 & 2.1 & 0.94 \\
\hline |prp-16--24.B-2 & 0.000 & 82 & 35 & 0.443 & 2376 & 57 & 2469 & 18 & 4 & .1613 & 1.05 & 9.913 & 3.0 & 0.446 & 2.8 & 0.94 \\
\hline prp-16--24.B-3 & 0.073 & 151 & 59 & 0.402 & 2600 & 37 & 2639 & 13 & 1 & .1785 & 0.77 & 12.222 & 1.9 & 0.497 & 1.8 & 0.92 \\
\hline prp-16--24.B-4 & 0.090 & 199 & 112 & 0.581 & 2637 & 36 & 2649 & 11 & 0 & .1796 & 0.66 & 12.515 & 1.8 & 0.505 & 1.7 & 0.93 \\
\hline prp-16--24.B-5 & 0.000 & 163 & 97 & 0.616 & 2659 & 38 & 2663 & 11 & 0 & 1811 & 0.66 & 12.748 & 1.9 & 0.511 & 1.7 & 0.93 \\
\hline prp-16--24.B-6 & 0.994 & 58 & 37 & 0.663 & 2700 & 53 & 2569 & 32 & -5 & 1712 & 1.92 & 12.275 & 3.1 & 0.520 & 2.4 & 0.78 \\
\hline prp-16--24.B-7 & 0.637 & 85 & 83 & 1.002 & 2100 & 38 & 2054 & 32 & -2 & .1268 & 1.83 & 6.731 & 2.8 & 0.385 & 2.1 & 0.76 \\
\hline prp-16--24.B-8 & 0.114 & 56 & 29 & 0.537 & 2560 & 52 & 2661 & 20 & 4 & .1809 & 1.19 & 12.157 & 2.7 & 0.487 & 2.5 & 0.90 \\
\hline prp-16--24.B-10 & 0.122 & 255 & 136 & 0.551 & 2705 & 32 & 2593 & 11 & -4 & .1736 & 0.67 & 12.482 & 1.6 & 0.521 & 1.5 & 0.91 \\
\hline prp-16--24.B-11 & 0.000 & 53 & 29 & 0.557 & 2581 & 54 & 2614 & 25 & 1 & .1759 & 1.52 & 11.940 & 3.0 & 0.492 & 2.6 & 0.86 \\
\hline prp-16-24.C.5 & 0.133 & 248 & 125 & 0.52 & 2546 & 33 & 2685 & 8 & 5 & .1835 & 0.49 & 12.255 & 1.6 & 0.484 & 1.6 & 0.95 \\
\hline prp-16-24C.3 & 0.034 & 416 & 279 & 0.69 & 2294 & 28 & 2587 & 7 & 11 & .1730 & 0.40 & 10.196 & 1.5 & 0.427 & 1.4 & 0.96 \\
\hline prp-16-24C.4 & 0.222 & 92 & 91 & 1.02 & 2658 & 43 & 2858 & 25 & 7 & .2040 & 1.55 & 14.350 & 2.5 & 0.510 & 2.0 & 0.78 \\
\hline prp-16-24.C.5 & 0.000 & 57 & 118 & 2.133 & 2056 & 42 & 2094 & 26 & 2 & .1297 & 1.49 & 6.719 & 2.8 & 0.376 & 2.4 & 0.85 \\
\hline prp-16-24C. 6 & 0.028 & 102 & 54 & 0.55 & 2428 & 40 & 2574 & 14 & 6 & .1716 & 0.81 & 10.821 & 2.1 & 0.457 & 2.0 & 0.92 \\
\hline prp-16-24C.7 & 0.049 & 153 & 118 & 0.80 & 2157 & 33 & 2451 & 13 & 12 & .1596 & 0.77 & 8.741 & 2.0 & 0.397 & 1.8 & 0.92 \\
\hline prp-16-24C.9 & 0.362 & 107 & 61 & 0.59 & 2439 & 40 & 2551 & 19 & 4 & . 1694 & 1.11 & 10.739 & 2.3 & 0.460 & 2.0 & 0.87 \\
\hline prp-16-24.C.10 & 0.000 & 103 & 57 & 0.576 & 2614 & 39 & 2680 & 14 & 2 & .1830 & 0.87 & 12.621 & 2.0 & 0.500 & 1.8 & 0.90 \\
\hline prp-16-24.C.11 & 0.037 & 101 & 58 & 0.593 & 2497 & 46 & 2546 & 19 & 2 & .1688 & 1.13 & 11.007 & 2.5 & 0.473 & 2.2 & 0.89 \\
\hline prp-16-24C.12 & 0.110 & 482 & 208 & 0.45 & 1745 & 23 & 2001 & 22 & 13 & .1231 & 1.26 & 5.274 & 1.9 & 0.311 & 1.5 & 0.76 \\
\hline prp-16-24.C.15 & 0.043 & 89 & 36 & 0.418 & 2661 & 43 & 2689 & 16 & 1 & .1840 & 0.99 & 12.962 & 2.2 & 0.511 & 2.0 & 0.89 \\
\hline prp-16-24.C.16 & 0.000 & 166 & 123 & 0.761 & 2050 & 27 & 2073 & 17 & 1 & .1282 & 0.94 & 6.616 & 1.8 & 0.374 & 1.6 & 0.86 \\
\hline prp-16-24.C.17 & 0.080 & 91 & 49 & 0.549 & 2708 & 43 & 2609 & 16 & -4 & .1753 & 0.98 & 12.617 & 2.2 & 0.522 & 1.9 & 0.89 \\
\hline prp-16-24.C.18 & 0.000 & 160 & 77 & 0.498 & 2616 & 79 & 2687 & 35 & 3 & .1838 & 2.14 & 12.681 & 4.3 & 0.500 & 3.7 & 0.87 \\
\hline prp-16-24.C.19 & 0.197 & 520 & 334 & 0.665 & 1907 & 19 & 2866 & 8 & 33 & .2049 & 0.49 & 9.726 & 1.2 & 0.344 & 1.1 & 0.92 \\
\hline prp-16-24.C.20 & 0.207 & 255 & 160 & 0.649 & 2423 & 27 & 2604 & 11 & 7 & .1748 & 0.65 & 10.996 & 1.5 & 0.456 & 1.3 & 0.90 \\
\hline prp-16-24.C.21 & 0.127 & 81 & 47 & 0.599 & 2670 & 43 & 2645 & 17 & -1 & .1791 & 1.05 & 12.669 & 2.2 & 0.513 & 2.0 & 0.88 \\
\hline prp-16-24.C.25 & 0.000 & 145 & 59 & 0.421 & 2067 & 29 & 2052 & 20 & -1 & .1266 & 1.12 & 6.599 & 2.0 & 0.378 & 1.6 & 0.82 \\
\hline prp-16-24.C.26 & 0.000 & 312 & 104 & 0.344 & 1988 & 22 & 2084 & 11 & 5 & .1290 & 0.65 & 6.425 & 1.4 & 0.361 & 1.3 & 0.89 \\
\hline prp-16-24C.27 & 0.272 & 279 & 194 & 0.72 & 2327 & 30 & 2471 & 17 & 6 & .1615 & 1.02 & 9.676 & 1.8 & 0.435 & 1.5 & 0.83 \\
\hline prp-16-24C.28 & 0.111 & 362 & 229 & 0.65 & 2243 & 28 & 2581 & 15 & 13 & .1724 & 0.92 & 9.897 & 1.7 & 0.416 & 1.5 & 0.85 \\
\hline рrp-16-24С.29 & 0.089 & 85 & 31 & 0.37 & 2462 & 73 & 2635 & 14 & 7 & .1781 & 0.85 & 11.423 & 3.6 & 0.465 & 3.5 & 0.97 \\
\hline prp-16-24C.30 & 0.211 & 215 & 124 & 0.59 & 2634 & 35 & 2688 & 16 & 2 & .1838 & 0.98 & 12.796 & 1.9 & 0.505 & 1.6 & 0.85 \\
\hline prp-16-24С.31 & 0.205 & 370 & 239 & 0.67 & 2471 & 30 & 2636 & 29 & 6 & .1782 & 1.72 & 11.478 & 2.3 & 0.467 & 1.5 & 0.65 \\
\hline prp-16-24.C.32 & 0.140 & 153 & 80 & 0.537 & 2533 & 34 & 2656 & 13 & 5 & .1804 & 0.79 & 11.968 & 1.8 & 0.481 & 1.6 & 0.90 \\
\hline prp-16-24.C.33 & 0.018 & 253 & 72 & 0.293 & 3168 & 34 & 3246 & 7 & 2 & .2599 & 0.44 & 22.742 & 1.4 & 0.635 & 1.3 & 0.95 \\
\hline prp-16-24.C.35 & 0.079 & 96 & 65 & 0.702 & 2642 & 109 & 2626 & 16 & -1 & .1771 & 0.98 & 12.372 & 5.1 & 0.507 & 5.0 & 0.98 \\
\hline prp-16-24.С.36 & 0.032 & 356 & 212 & 0.615 & 2606 & 26 & 2651 & 8 & 2 & .1798 & 0.51 & 12.345 & 1.3 & 0.498 & 1.2 & 0.92 \\
\hline prp-16-24.C.37 & 0.185 & 100 & 76 & 0.787 & 2598 & 40 & 2615 & 17 & 1 & .1760 & 1.00 & 12.044 & 2.1 & 0.496 & 1.9 & 0.88 \\
\hline prp-16-24С.38 & 0.055 & 271 & 141 & 0.54 & 2503 & 33 & 2635 & 8 & 5 & .1780 & 0.50 & 11.649 & 1.7 & 0.475 & 1.6 & 0.95 \\
\hline
\end{tabular}

Errors are 1-sigma; $\mathrm{Pb}_{\mathrm{c}}$ and $\mathrm{Pb}^{*}$ indicate the common and radiogenic portions, respectively.

Error in Standard calibration was $0.43 \%$ (not included in above errors but required when comparing data from different mounts).

(1) Common $\mathrm{Pb}$ corrected using measured ${ }^{204} \mathrm{~Pb}$. 
Table 4 (cont). Summary of SHRIMP U-Pb zircon data for samples PRP-16 (Rio Preto Quarry) and PEC01 (Pecuária Cave).

\begin{tabular}{|c|c|c|c|c|c|c|c|c|c|c|c|c|c|c|c|c|}
\hline Spot Name & $\begin{array}{l}\text { (1) } \% \\
{ }^{206} \mathrm{~Pb}_{c}\end{array}$ & ppm & $\begin{array}{l}\mathrm{ppm} \\
\text { Th }\end{array}$ & $\begin{array}{l}{ }^{232} \mathrm{Th} \\
{ }^{238} \mathrm{U}\end{array}$ & $\begin{array}{c}(1) \\
{ }^{206} \mathrm{~Pb} \\
{ }^{238} \mathrm{U} \\
\text { Age }\end{array}$ & $\begin{array}{l}1 \mathrm{~s} \\
\text { err }\end{array}$ & $\begin{array}{c}(1) \\
{ }^{207} \mathrm{~Pb} \\
{ }^{206} \mathrm{~Pb} \\
\text { Age }\end{array}$ & $\begin{array}{l}1 \mathrm{~s} \\
\text { err }\end{array}$ & $\begin{array}{c}\% \\
\text { Dis- } \\
\text { cor- } \\
\text { dant }\end{array}$ & $\begin{array}{l}\text { (1) }{ }^{207} \mathrm{~Pb}^{*} \\
\mathrm{p}^{206} \mathrm{~Pb}^{*}\end{array}$ & $\begin{array}{l}\% \\
\text { err }\end{array}$ & $\begin{array}{c}\text { (1) }{ }^{207} \mathrm{~Pb}^{\star} \\
{ }^{235} \mathrm{U}\end{array}$ & $\begin{array}{l}\% \\
\text { err }\end{array}$ & $\begin{array}{c}\text { (1) }{ }^{206} \mathrm{~Pb}^{*} \\
{ }^{238} \mathrm{U}\end{array}$ & $\begin{array}{l}\% \\
\text { err }\end{array}$ & $\begin{array}{l}\text { err } \\
\text { corr }\end{array}$ \\
\hline
\end{tabular}

Sample PEC-01

\begin{tabular}{|c|c|c|c|c|c|c|c|c|c|c|c|c|c|c|c|c|}
\hline PEC-1.1 & 2.54 & 536 & 209 & 0.40 & 931 & 23 & 1596 & 68 & 71 & .0985 & 3.7 & 2.111 & 4.5 & 0.155 & 2.6 & .59 \\
\hline PEC-2.1 & 0.31 & 70 & 38 & 0.56 & 2113 & 51 & 2107 & 22 & 0 & .1307 & 1.3 & 6.989 & 3.1 & 0.388 & 2.8 & .91 \\
\hline PEC-3.1 & 0.87 & 44 & 60 & 1.40 & 1514 & 40 & 1482 & 107 & -2 & .0927 & 5.7 & 3.384 & 6.4 & 0.265 & 3.0 & .47 \\
\hline PEC-4.1 & 0.79 & 69 & 60 & 0.90 & 2630 & 61 & 2705 & 25 & 3 & .1858 & 1.5 & 12.904 & 3.2 & 0.504 & 2.8 & .88 \\
\hline PEC-5.1 & 0.17 & 140 & 86 & 0.63 & 3241 & 69 & 3294 & 8 & 2 & .2679 & 0.5 & 24.130 & 2.7 & 0.653 & 2.7 & .98 \\
\hline PEC-6.1 & 1.83 & 531 & 1001 & 1.95 & 1250 & 32 & 1573 & 50 & 26 & .0973 & 2.7 & 2.871 & 3.9 & 0.214 & 2.8 & .72 \\
\hline PEC-7.1 & 0.59 & 84 & 76 & 0.93 & 2141 & 52 & 2087 & 36 & -3 & .1292 & 2.0 & 7.018 & 3.5 & 0.394 & 2.8 & .81 \\
\hline PEC-8.1 & 0.71 & 83 & 59 & 0.73 & 2611 & 59 & 2620 & 19 & 0 & .1765 & 1.2 & 12.150 & 3.0 & 0.499 & 2.8 & .92 \\
\hline PEC-9.1 & 0.33 & 99 & 135 & 1.40 & 2815 & 62 & 2815 & 13 & 0 & .1987 & 0.8 & 14.999 & 2.8 & 0.548 & 2.7 & .96 \\
\hline PEC-10.1 & 0.35 & 251 & 301 & 1.24 & 2720 & 59 & 2896 & 8 & 6 & .2087 & 0.5 & 15.106 & 2.7 & 0.525 & 2.6 & .98 \\
\hline PEC-11.1 & 2.42 & 31 & 31 & 1.03 & 1628 & 45 & 1595 & 178 & -2 & .0985 & 9.5 & 3.900 & 10.0 & 0.287 & 3.1 & .31 \\
\hline PEC-12.1 & 0.83 & 194 & 317 & 1.69 & 2445 & 54 & 2587 & 15 & 6 & .1730 & 0.9 & 11.003 & 2.8 & 0.461 & 2.7 & .95 \\
\hline PEC-13.1 & 0.26 & 232 & 136 & 0.61 & 2615 & 57 & 2642 & 9 & 1 & .1789 & 0.6 & 12.338 & 2.7 & 0.500 & 2.6 & .98 \\
\hline PEC-14.1 & 0.89 & 119 & 82 & 0.72 & 1511 & 37 & 1494 & 46 & -1 & .0933 & 2.4 & 3.400 & 3.6 & 0.264 & 2.7 & .75 \\
\hline PEC-15.1 & 0.23 & 156 & 125 & 0.83 & 2686 & 59 & 2626 & 11 & -2 & .1772 & 0.7 & 12.628 & 2.8 & 0.517 & 2.7 & .97 \\
\hline PEC-16.1 & 0.83 & 103 & 96 & 0.97 & 1818 & 43 & 1770 & 49 & -3 & .1083 & 2.7 & 4.863 & 3.9 & 0.326 & 2.7 & .71 \\
\hline PEC-17.1 & 1.62 & 104 & 165 & 1.63 & 2023 & 48 & 2033 & 44 & 0 & .1253 & 2.5 & 6.368 & 3.7 & 0.369 & 2.7 & .74 \\
\hline PEC-28.1 & 1.11 & 254 & 395 & 1.61 & 2034 & 47 & 2840 & 17 & 40 & .2017 & 1.0 & 10.314 & 2.9 & 0.371 & 2.7 & .94 \\
\hline PEC-29.1 & 0.31 & 157 & 149 & 0.98 & 2039 & 85 & 2370 & 22 & 16 & .1522 & 1.3 & 7.807 & 5.0 & 0.372 & 4.9 & .97 \\
\hline PEC-30.1 & 0.34 & 103 & 134 & 1.35 & 1774 & 43 & 1791 & 34 & 1 & .1095 & 1.9 & 4.783 & 3.4 & 0.317 & 2.8 & .83 \\
\hline PEC-31.1 & 0.91 & 96 & 43 & 0.46 & 2388 & 55 & 2417 & 23 & 1 & .1564 & 1.3 & 9.671 & 3.1 & 0.448 & 2.8 & .90 \\
\hline PEC-34.1 & 0.46 & 130 & 84 & 0.67 & 1825 & 43 & 1776 & 25 & -3 & .1086 & 1.4 & 4.901 & 3.0 & 0.327 & 2.7 & .89 \\
\hline PEC-18.1 & 0.46 & 130 & 84 & 0.67 & 1134 & 28 & 1776 & 25 & 57 & .1086 & 1.4 & 2.879 & 3.0 & 0.192 & 2.7 & .89 \\
\hline PEC-18.1 & 2.18 & 518 & 358 & 0.71 & 1068 & 28 & 1697 & 71 & 59 & .1040 & 3.9 & 2.583 & 4.8 & 0.180 & 2.8 & .59 \\
\hline PEC-19.1 & 0.52 & 526 & 265 & 0.52 & 2384 & 53 & 3166 & 9 & 33 & .2471 & 0.6 & 15.248 & 2.7 & 0.448 & 2.7 & .98 \\
\hline PEC-20.1 & 0.28 & 235 & 145 & 0.64 & 2189 & 49 & 2137 & 13 & -2 & .1329 & 0.8 & 7.409 & 2.8 & 0.404 & 2.7 & .96 \\
\hline PEC-21.1 & 0.26 & 130 & 69 & 0.54 & 1519 & 37 & 1498 & 36 & -1 & .0935 & 1.9 & 3.426 & 3.3 & 0.266 & 2.7 & .82 \\
\hline PEC-22.1 & 0.57 & 90 & 88 & 1.02 & 2091 & 49 & 2068 & 30 & -1 & .1278 & 1.7 & 6.752 & 3.3 & 0.383 & 2.8 & .85 \\
\hline PEC-36.1 & 1.54 & 104 & 89 & 0.88 & 1742 & 44 & 1762 & 58 & 1 & .1078 & 3.2 & 4.610 & 4.3 & 0.310 & 2.9 & .67 \\
\hline PEC-37.1 & 0.81 & 245 & 226 & 0.95 & 1784 & 42 & 1794 & 32 & 1 & .1097 & 1.8 & 4.823 & 3.3 & 0.319 & 2.7 & .84 \\
\hline PEC-39.1 & 0.84 & 165 & 329 & 2.06 & 2824 & 62 & 2983 & 12 & 6 & .2203 & 0.7 & 16.692 & 2.8 & 0.550 & 2.7 & .96 \\
\hline PEC-40.1 & 0.53 & 197 & 99 & 0.52 & 3070 & 65 & 3194 & 9 & 4 & .2514 & 0.6 & 21.147 & 2.7 & 0.610 & 2.7 & .98 \\
\hline PEC-42.1 & 3.11 & 462 & 547 & 1.22 & 1512 & 36 & 1925 & 73 & 27 & .1180 & 4.1 & 4.298 & 4.9 & 0.264 & 2.7 & .55 \\
\hline PEC-43.1 & 3.11 & 462 & 547 & 1.22 & 2255 & 52 & 1925 & 73 & -15 & .1180 & 4.1 & 6.812 & 4.9 & 0.419 & 2.7 & .56 \\
\hline PEC-44.1 & 0.55 & 346 & 298 & 0.89 & 1808 & 43 & 1882 & 18 & 4 & .1151 & 1.0 & 5.140 & 2.9 & 0.324 & 2.7 & .94 \\
\hline PEC-45.1 & 1.55 & 99 & 88 & 0.92 & 1960 & 46 & 2403 & 33 & 23 & .1551 & 2.0 & 7.600 & 3.4 & 0.355 & 2.7 & .81 \\
\hline PEC-46.1 & 0.63 & 163 & 49 & 0.31 & 2138 & 49 & 2119 & 30 & -1 & .1315 & 1.7 & 7.134 & 3.2 & 0.393 & 2.7 & .85 \\
\hline PEC-47.1 & 0.16 & 243 & 122 & 0.52 & 2882 & 61 & 2929 & 9 & 2 & .2131 & 0.6 & 16.559 & 2.7 & 0.564 & 2.6 & .98 \\
\hline PEC-48.1 & 0.56 & 81 & 41 & 0.53 & 1864 & 46 & 1810 & 48 & -3 & .1106 & 2.7 & 5.113 & 3.9 & 0.335 & 2.8 & .73 \\
\hline PEC-49.1 & 1.20 & 206 & 120 & 0.60 & 1477 & 35 & 1386 & 49 & -6 & .0882 & 2.5 & 3.129 & 3.7 & 0.257 & 2.7 & .73 \\
\hline PEC-50.1 & 1.22 & 231 & 160 & 0.71 & 1435 & 35 & 1907 & 37 & 33 & .1168 & 2.0 & 4.012 & 3.4 & 0.249 & 2.7 & .80 \\
\hline PEC-52.1 & 1.16 & 321 & 360 & 1.16 & 1365 & 33 & 1743 & 33 & 28 & .1067 & 1.8 & 3.468 & 3.2 & 0.236 & 2.6 & .82 \\
\hline PEC-53.1 & 0.71 & 136 & 85 & 0.64 & 3334 & 70 & 3478 & 13 & 4 & .3016 & 0.8 & 28.158 & 2.8 & 0.677 & 2.7 & .95 \\
\hline PEC-55.1 & 0.38 & 129 & 98 & 0.78 & 2633 & 58 & 2547 & 18 & -3 & .1689 & 1.1 & 11.750 & 2.9 & 0.504 & 2.7 & .93 \\
\hline PEC-56.1 & 0.17 & 224 & 120 & 0.56 & 3379 & 70 & 3290 & 6 & -3 & .2672 & 0.4 & 25.384 & 2.7 & 0.689 & 2.7 & .99 \\
\hline PEC-57.1 & 2.77 & 62 & 84 & 1.40 & 2373 & 57 & 2557 & 46 & 8 & .1699 & 2.8 & 10.427 & 4.0 & 0.445 & 2.9 & .72 \\
\hline PEC-58.1 & 0.04 & 250 & 232 & 0.96 & 2751 & 59 & 2651 & 8 & -4 & .1798 & 0.5 & 13.196 & 2.7 & 0.532 & 2.6 & .99 \\
\hline PEC-59.1 & 0.36 & 110 & 115 & 1.08 & 2150 & 52 & 2104 & 21 & -2 & .1305 & 1.2 & 7.123 & 3.1 & 0.396 & 2.9 & .92 \\
\hline PEC-60.1 & -0.03 & 72 & 74 & 1.05 & 2711 & 62 & 2623 & 15 & -3 & .1768 & 0.9 & 12.743 & 2.9 & 0.523 & 2.8 & .95 \\
\hline PEC-61.1 & 0.67 & 163 & 105 & 0.66 & 2569 & 57 & 2616 & 17 & 2 & .1760 & 1.0 & 11.883 & 2.9 & 0.490 & 2.7 & .93 \\
\hline PEC-62.1 & 1.12 & 223 & 123 & 0.57 & 2044 & 47 & 2368 & 22 & 16 & .1520 & 1.3 & 7.821 & 3.0 & 0.373 & 2.7 & .90 \\
\hline PEC-63.1 & 0.90 & 80 & 49 & 0.63 & 3309 & 72 & 3271 & 13 & -1 & .2641 & 0.8 & 24.424 & 2.9 & 0.671 & 2.8 & .96 \\
\hline PEC-64.1 & 0.51 & 94 & 61 & 0.67 & 2234 & 52 & 2163 & 26 & -3 & .1350 & 1.5 & 7.708 & 3.1 & 0.414 & 2.8 & .88 \\
\hline PEC-65.1 & 0.07 & 81 & 83 & 1.05 & 2148 & 51 & 2078 & 20 & -3 & .1285 & 1.1 & 7.007 & 3.0 & 0.395 & 2.8 & .93 \\
\hline PEC-66.1 & 0.76 & 53 & 30 & 0.59 & 3340 & 75 & 3291 & 16 & -1 & .2675 & 1.0 & 25.039 & 3.1 & 0.679 & 2.9 & .94 \\
\hline
\end{tabular}


A)
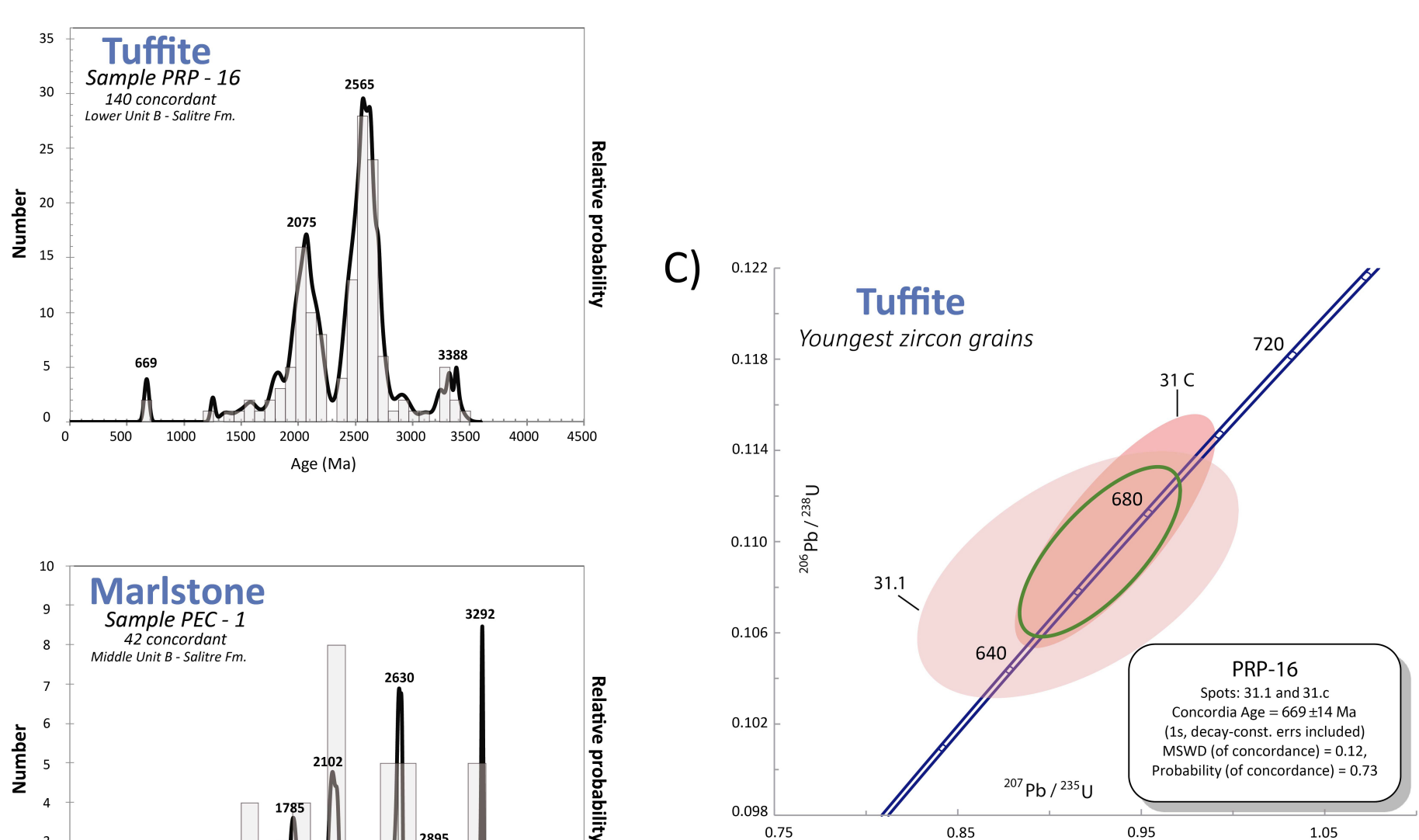

B)

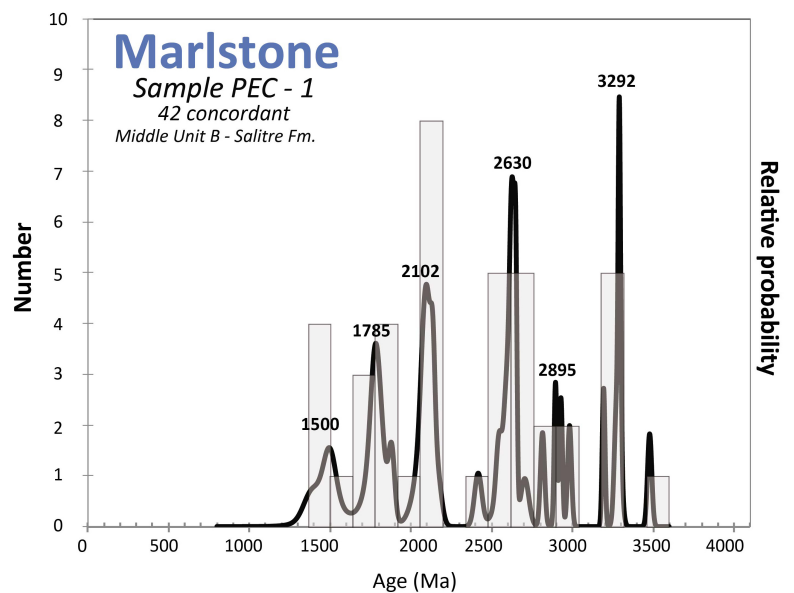

Figura 28. Histogram with U-Pb ages from detrital zircons of Salitre Fm., Irecê Basin, Bahia

(samples PRP-16 and PEC-1). 


\subsection{DISCUSSION}

A synthesis of the stratigraphic, petrologic and geochronologic data presented herein allows derivation of the depositional model, provenance and its implications for west Gondwana evolution.

\subsubsection{SETTINGS AND PALEOENVIRONMENTAL PARAMETERS}

Mud-dominated carbonate deposits with geometry essentially tabular and packages with extension lateral from several tens to hundreds of meters has been described and related the deepwater ramp in different basins formed in an intracratonic setting (Calvet and Tucker, 1988; Burchette and Wright, 1992; Thomson et al., 2014). Especially regarding the carbonate sequences of a post- Sturtian cap carbonate, is very common the establishment of carbonate platform and abundant examples in literature the geological record with such characteristics in Brazil and worldwide (Vieira et al., 2007; and references therein). However, the definition of paleobathymetry is a difficult task due absence of the fossiliferous assembly. Nevertheless, based on the facies zones model of carbonate ramp well established in the literature (e.g. Burchette and Wright, 1992), and guided by sedimentological data previously described, it is suggested of at least three different depositional zones: basin, outer ramp and middle ramp. The basin zone is characterized by $\mathrm{Fl}$ and $\mathrm{Fw}$ organic facies, interpreted as hemipelagic sedimentation in the context in which dominate water oxygen impoverishment conditions, above pycno-/thermocline level (Flügel, 2010). After, it is the outer ramp is composed by facies $\mathrm{He}, \mathrm{Mm}$ and $\mathrm{FI}(\mathrm{v})$ proposed occurrence of outer ramp zone, primarily pointing the transition to zones well-oxygenated waters represents by facies He. The presence of wave 
ripples at distal portion of outer ramp may indicate occasional storm wave action (Bádenas and Aurell, 2001). However, in the outer ramp prevails mud sedimentation on welloxygenated waters circumstances, herein represented by facies $\mathrm{Mm}$. This facies indicate deeper waters typically below wave base (e.g. Bahamonde et al., 2004) that which indicates microbial boundstone is not restricted to the euphotic zones. Facies $\mathrm{FI}$ (v) suggest active volcanism during mud sedimentation. The middle ramp is characterized by facies WP and Lm, being interpreted as deposition in the context in which dominates bottom hydrodynamics conditions low to moderate. Although facies $\mathrm{Lm}$ can be occurs both shallow and deeper water settings (Grotzinger and Al-Rawahi, 2014), the remarkable decreases of the terrigenous content and clearer colors are factors highly suggestive of have been deposited in the context of shallow water.

\subsubsection{ISOTOPE CHEMOSTRATIGRAPHY}

Isotopic techniques, especially those related to the variation of stable isotopes as $\delta^{13} \mathrm{C}$ values, have proved to be important tool in research on paleoenvironment and paleoclimate. Stable isotopes as $\delta^{18} \mathrm{O}$ values are easily changed by weathering processes and Neoproterozoic records are usually disposed of paleoenvironmental analysis (Sharp, 2007). Commonly applied in Neoproterozoic carbonate sequences such studies have applications mainly to discussions on the environmental conditions at the time of deposition, and to qualitative evaluation in the processes post-depositional changes. Another common application has been for indirect dating: In the absence of fossils or geochronological data, $\delta^{13} \mathrm{C}$ variation trends had guided chronostratigraphic correlations, because it is admitted as a datum of negative anomalies related to glacial events well documented in Brazil and worldwide, 
according to research carried out by different authors (Misi et al., 2007; Halverson et al., 2010; Alvarenga et al., 2014; and others).

More recently, the applications of this technique for extrabasinal correlations have been questioned, and even discouraged (Frimmel, 2010). This author suggests that carbon isotope age curves constructed from Neoproterozoic seawater reveals a high level of inconsistency, then it is not appropriate to use for indirect dating. However, the use of such isotope in order to infer the reservoir is possible and regarding the isotope composition of the depositional environment. Studies based on isotopic techniques require prior evaluation of data that should be the original signal of the isotopic record (Brand and Veizer, 1980). Thus, it becomes imperative to use estimation of the preservation of samples. Usually this estimatation is conducted from the analysis of $\mathrm{Mn} / \mathrm{Sr}$ ratios and $\mathrm{Sr}$ concentration $(\geq 300$ ppm), and the best samples are the ones that have high Sr and low Mn contents (Brand and Veizer, 1980; Kaufman and Knoll, 1995; Misi and Veizer, 1998).

However, the high Mn content (>200 ppm) could suggest a proxy for seawater (Halverson et al., 2005), constituting the original isotopic signal. Alternatively, the isotope signal can be modified by post- depositional events from diagenetic or hydrothermal fluid interaction with solutions enriched in this element (Lin and Taillefert, 2014). Considering the parameters defended in Veizer (1983), only four samples of our sample set are useful as primary and adequate to analyze the isotopic signature of depositional environment (Table 3). The well preserved samples show slight negative $\delta^{13} \mathrm{C}$ values ranging from -0,99 \%о (sample PRP-15; Tab. 2) to lower negative values, between $-0,37$ and $-0,22 \%$, recorded in the middle ramp 
deposits. The other analyzed samples present siliciclastic sediments on matrix admixture, verified in field and petrographic observations. All samples are just overlying the cap carbonates (Fig. 21) and show slight negative $\delta^{13} \mathrm{C}$ values, with isotopic signatures as provided in the literature (e.g., Misi et al., 1998).

\subsubsection{PROVEnANCE DETERMINATIONS}

We distinguish two set of zircon age distribution in the platform ramp of the Irecê Basin. The first one, which it is positioned at base of Unit B (Rio Preto Quarry; Supplementary Geospatial data; Fig. 23) is marked by a dominant Archean ages, with peaks at 3.38 and 2.56 $\mathrm{Ga}$, and Paleoproterozoic ages with peak at $2.07 \mathrm{Ga}$. There is a presence of a younger age of $669 \pm 14 \mathrm{Ma}$ Ga which it is interpreted as maximum depositional age for the carbonate platform. Toulkeridis et al. (1999) 700.9 $\pm 5.5 \mathrm{Ma}$ (Rb-Sr isochron age) of authigenic chlorite applied to clay minerals of black limestone collected from unit B of the Salitre Fm, in the basal section of the Rio Preto Quarry (personal comm. by M. Babinski). The present results suggest that the depositional age of the platform basal section of the Salitre Fm. (or Unit B after Misi \& Veizer, 1998) is between $700.9 \pm 5.5 \mathrm{Ma}$ ans $669 \pm 14 \mathrm{Ma}$.

The overlying layer, situated at Pecuária Cave (PEC-1; Supplementary Geospatial data; Fig. 23) display a different zircon pattern distribution with Archean (3.29, 2,89 and $2.63 \mathrm{Ga}$ ), Paleoproterozoic (2.10 Ga), Statherian (1.78 Ga) and Calymmian (1.50 Ga) peaks. These ages distribution is similar to the subjacent Mesoproterozoic rocks of the Espinhaço Supergroup as already discussed by Guadagnin et al. (2015b) and Guadagnin and Chemale Jr. (2015). The zircon grains with these age peaks are interpreted to be derived from the denudation of 
Gavião Block, an Archean Paleoplate (Barbosa and Sabaté, 2004) and/or the Lower and Middle Espinhaço Sequences (Chemale et al., 2012; Guadagnin et al., 2015a). Provenance analysis of Chapada Diamantina region, including Espinhaço Supergroup (1.8 to 0.9 Ga) and Bebedouro Formation (< ca. $875 \mathrm{Ma}$ ), reveals local sources for the sediments (Figueiredo et al., 2009; Guadagnin et al., 2015b). Derivation is related to either the Gavião Block within basement of SFC (> ca. 2.0 Ga); volcanism at ca. $1.75 \mathrm{Ga}, 1.5 \mathrm{Ga}$ and $1.38 \mathrm{Ga}$ (Rio dos Remédios Group, Bomba and Tombador formations, respectively); and/or recycled sediments (Guadagnin et al., 2015a; b).

If we compare the age distribution of the two analyzed samples with the subjacent units, namely Middle and Upper Espinhaço sequences (Guadagnin et al., 2015b; Guadagnin and Chemale, 2015) and Bebedouro Fm. (Figuereido et al., 2009; Santos et al., 2011), the basal unit located at the Rio Preto quarry (sample PRP-16) present a similar age peaks as those of the Lower Tombador (Middle Espinhaço Sequence). On other side, the upper unit (Middle Unit B, sample PEC-1) has a zircon distribution similar to the Upper Tombador Fm. (Upper Espinhaço Sequence) and Bebedouro Fm. (Fig. 29). The present data suggest that the Lower Unit B is mostly derived of Lower Tombador Fm. units, as sedimentary recycling process. However the zircon age distribution of the Middle Unit B reproduce that pattern of Upper Tombador and Bebedouro formations, so that we interpret that probable the Bebedouro units were source area for the Middle Unit B, while during the deposition Lower Unit B the Bebedouro did not outcrop in the studied area. 


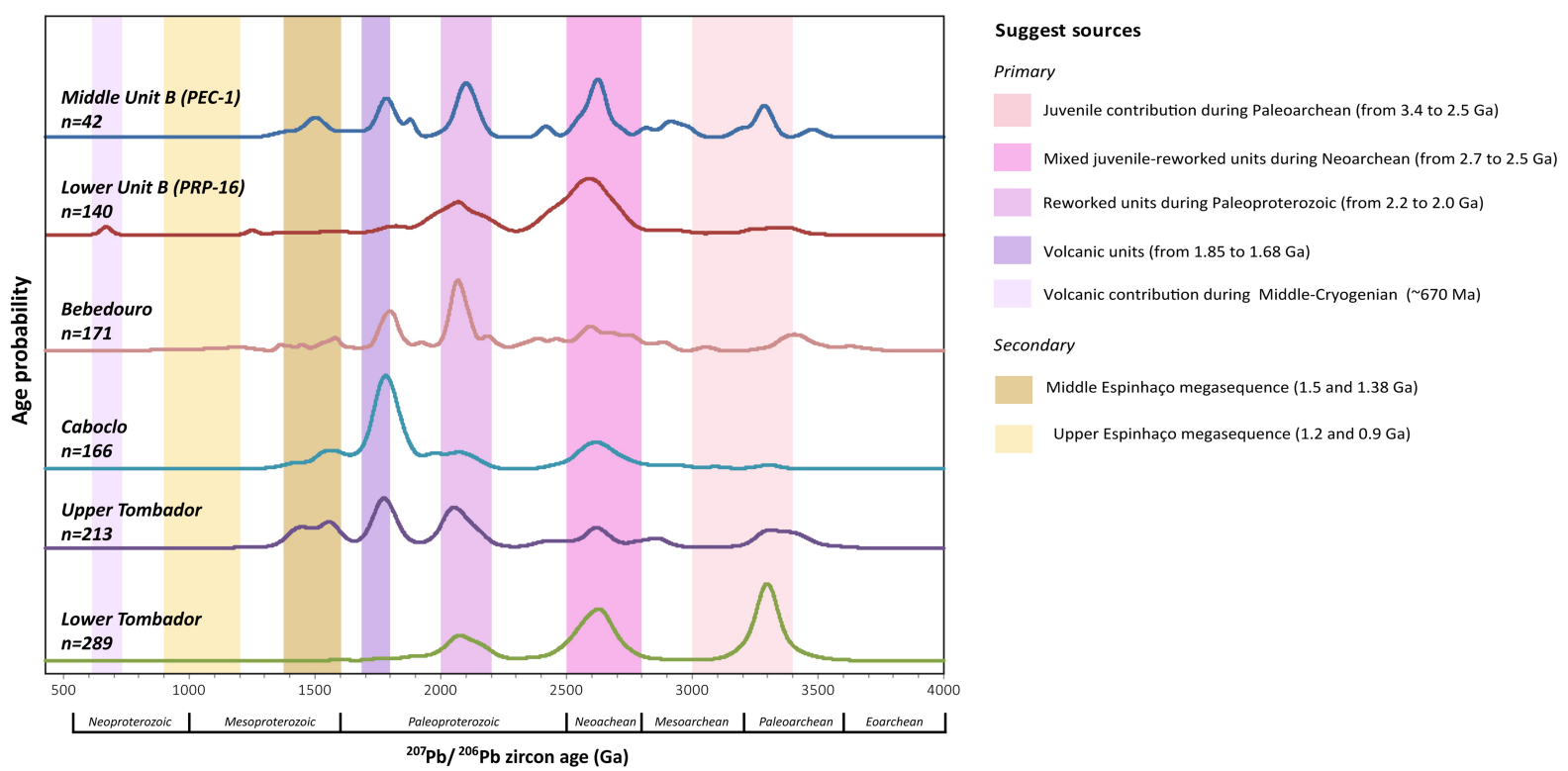

Figura 29. Normalized age probability histogram of the detrital zircon U-Pb ages from

Salitre Formation (Samples PRP-16 and PEC-1; this study), Bebedouro Formation (after Santos, 2011 and Figueiredo et al., 2009), Caboclo Formation and Upper and Lower Tombador Formation (after Guadagnin et al., 2015b).

\subsubsection{IRECÊ BASIN FILLING AND WEST GONDWANA EVOLUTION}

The basal section of Irecê Basin is formed by the Bebedouro Fm. and Units C and B (Figure 2) which corresponds to a gulf area similar to the Mexico Gulf, deposited on the São Francisco Craton units during the Neoproterozoic. It is interpreted as gulf because the sedimentation occurred on the basement in continental environment and connected to a passive margin or a extension of ocean into the São Francisco Paleoplate (see below). The basement rocks of the Irecê Basin are the intracratonic sedimentary rocks of the Espinhaço Supergroup (>0.93 to $1.8 \mathrm{Ga}$ ), Paleoproterozoic to Archean Terranes of the Gavião Block. The Bebedouro Fm. and Units C and B of the Irecê Basin (Fig. 22) were connected to a rift-related passive margin well documented in the Rio Preto, Riacho do Pontal, Sergipana and Araçuaí Belts which 
started around 0.8 Ga to $0.7 \mathrm{Ga}$ (Pedrosa-Soares et al., 2008; Oliveira et al., 2010; Caxito and Uhlein, 2013, Caxito et al., 2014).

The locus of deposition of the SFS in the Chapada Diamantina area, where occurs the Irecê Basin, it was generated by an initial fragmentation process with generation of mafic dyke swarms, which evolved to rifting process during the Tonian rift stage (started at ca. $900 \mathrm{Ma}$; Silva et al., 1995). This process has been associated with break-up of the supercontinent Rodinia (e.g., Rosset et al., 2007; Evans et al., 2010; Silveira et al., 2013; and others). At study area, the first record of this igneous event is represented by dyke swarms dated at $934 \pm 14$ Ma (Loureiro et al., 2008), which intruded the entire Espinhaço Supergroup and does not cut the Bebedouro Fm. units.

Evidences of the mantle-activated rift system also has been related from others parts of the SFC (e.g., Pedro Lessa Suite, ca. 930 Ma; Dussin and Chemale, 2011), in the Congo, WestCongo Belt (Zanidian and Mayumbian Groups) and West African cratons, as well as others, and all are marked by large volumes of A-type bimodal magmatism considered anorogenic then related to intracratonic extensional stage (Pedrosa-Soares and Alckmin, 2011, and references therein).

Rio Preto Fold Belt, situated to NW of Chapada Diamantina area, is interpreted to have glacial deposits formed in rift-related environment, the Canabravinha Formation, which contains similar age distribution pattern of the Bebedouro Fm. and youngest zircon grains of $898 \pm 9.3 \mathrm{Ma}$ (Caxito et al., 2014). These authors suggest that this rifted margin evolved to an 
active margin (close to $600 \mathrm{Ma}$ ). The Araçuaí orogeny, part of the Araçuaí-West Congo orogen (AWCO; Alkmim et al., 2006), is located to the south of Chapada Diamantina. AWCO is described to have a complete Wilson Cycle with rifting processes started at $0.9 \mathrm{Ga}$, Early to Middle Cryogenian glacial deposits (Macaúbas Fm.), passive margin with Late Cryogenian to Ediacaran ophiolite slivers and the Ediacaran Rio Doce magmatic arc with orogenic basins.

The pre-, syn and post orogenic magmatism took place between $630 \mathrm{Ma}$ to $500 \mathrm{Ma}$ (e.g., Pedrosa-Soares et al., 2011; Alkmim et al., 2006). The Riacho do Pontal Fold Belt and Sergipana Fold Belt, are situated just northern and northeastern of Irecê Basin (Fig. 21B), respectively. They have similar geological evolution with passive margin developed between 800 to $640 \mathrm{Ma}$ and subduction related magmatism started at $630 \mathrm{Ma}$ and extended up to $570 \mathrm{Ma}$. This scenario suggests that Irecê Basin a gulf context was an extension inboard continent (Paleoplate São Francisco) of a widespread passive margin to the Northern, NW and NE segment developed from $\sim 0.8 \mathrm{Ga}$ to $\sim 0.64 \mathrm{Ma}$.

Tuffite beds, hence it is associated with mud-rocks and interpreted as formed by the incorporation of tuffaceous material into the water-lain sediment and suggests active volcanism during sedimentation. However, due to the zircon age distribution, the present tuffite contains dominant inherited Archean zircon ages (peaks at 3.38 and $2.56 \mathrm{Ga}$ ), and Paleoproterozoic ages (peak at $2.07 \mathrm{Ga}$ ). The presence of zircon formed at $669 \pm 14 \mathrm{Ma}$ that can be associated with any igneous activity at the continent or even passive margin of São Francisco-Congo Paleoplate. One main alternative would be late magmatic activity related to the blue sodalite syenites which intruded the basement of the SFC (some hundred $\mathrm{km}$ to 
south of study area) at as that described by $696 \pm 3$ Ma Da Rosa et al. (2007) and $685 \pm 3$ Ma Oliveira et al. 2014).

The platform carbonate of Unit B shows slightly negative $\delta{ }^{13} \mathrm{C}$ values and overlying cap carbonate rocks (Unit C, Fig. 22), located between the glacial-marine deposits of Bebedouro Fm. and the carbonate layers of lower Unit B, have strong negative $\delta 13 \mathrm{C}$ and is part of initial stage of deglaciation period. The present data suggests that glacial-marine deposits of Bebedouro Fm. probable formed during the Sturtian glacial epoch (ca. 717 - $660 \mathrm{Ma}$ ) which is covered by the cap dolomite in restricted basin scenario $\left(\delta^{13} \mathrm{C}\right.$ values up to $-6.04 \%$; Torquato and Misi, 1977). The same scenario has been identified in the Bambui Group, a pelite - carbonate cover of the São Francisco craton. Cap carbonates of the Sete Lagoas Formation, the lowermost unit of the Bambui Group, displays strong and regional negative $\delta{ }^{13} \mathrm{C}$ values (e.g. Iyer et al., 1995; Vieira et al. 2007) and yielded a $\mathrm{Pb}-\mathrm{Pb}$ isochron age of 740 \pm 22 Ma (Babinski et al., 2007), supporting a Middle Cryogenian-age for the underlying glacial deposits. The development of carbonate ramp (Unit B) occurred just after deposition of the cap dolomite, with mild conditions and negative $\delta{ }^{13} \mathrm{C}$ values close to zero, with deposition at $669 \pm 14 \mathrm{Ma}$ (Fig. 30). Our data support the interpretation of Misi et al. (2007) who interpreted the cap carbonate as a Sturtian cap carbonate. The present study suggests that the sedimentation rate in the carbonate ramp of Unit B was probably very low, and it was connected to an open ocean to the North and Northeast. Indeed, the passive margin sediments in the Riacho do Pontal and Sergipana mobile belts are represented by thicker 
sedimentary package deformed during the Brasiliano Cycle (Oliveira et al., 2010; Caxito and Uhlein, 2013) which probable had higher sedimentation rate.

In the intermediate section of the Unit B changes detrital zircon age patterns suggesting in this study an unconformity. Possible key factors controlling change of detrital zircon age patterns is associated with tectonic uplift with exposure of the Bebedouro glacial deposits. Indeed, the shift of detrital zircon age patterns suggests basin reorganization. Second-order sequences represent the major subdivisions of a first order sequence and reflect cycles of change in pattern observed at $10^{2}-\mathrm{m}$ scale. Similar to the Bambuí Group, which has a thick psamo-pelitic-carbonate sequence formed from $\leqslant 590$ to $\sim 500 \mathrm{Ma}$ in a foreland tectonic environment (e.g. Pimentel al, 2011, Paula-Santos et a., 2015), the carbonate of the Unit A (Cycle II; Fig. 22) of the Irecê Basin present also high positive $\delta{ }^{13} \mathrm{C}$. This unit probable represents the distal foreland deposits formed during the late to post-orogenic stage of Brasiliano Cycle of the Riacho Pontal and Sergipana fold belts. 


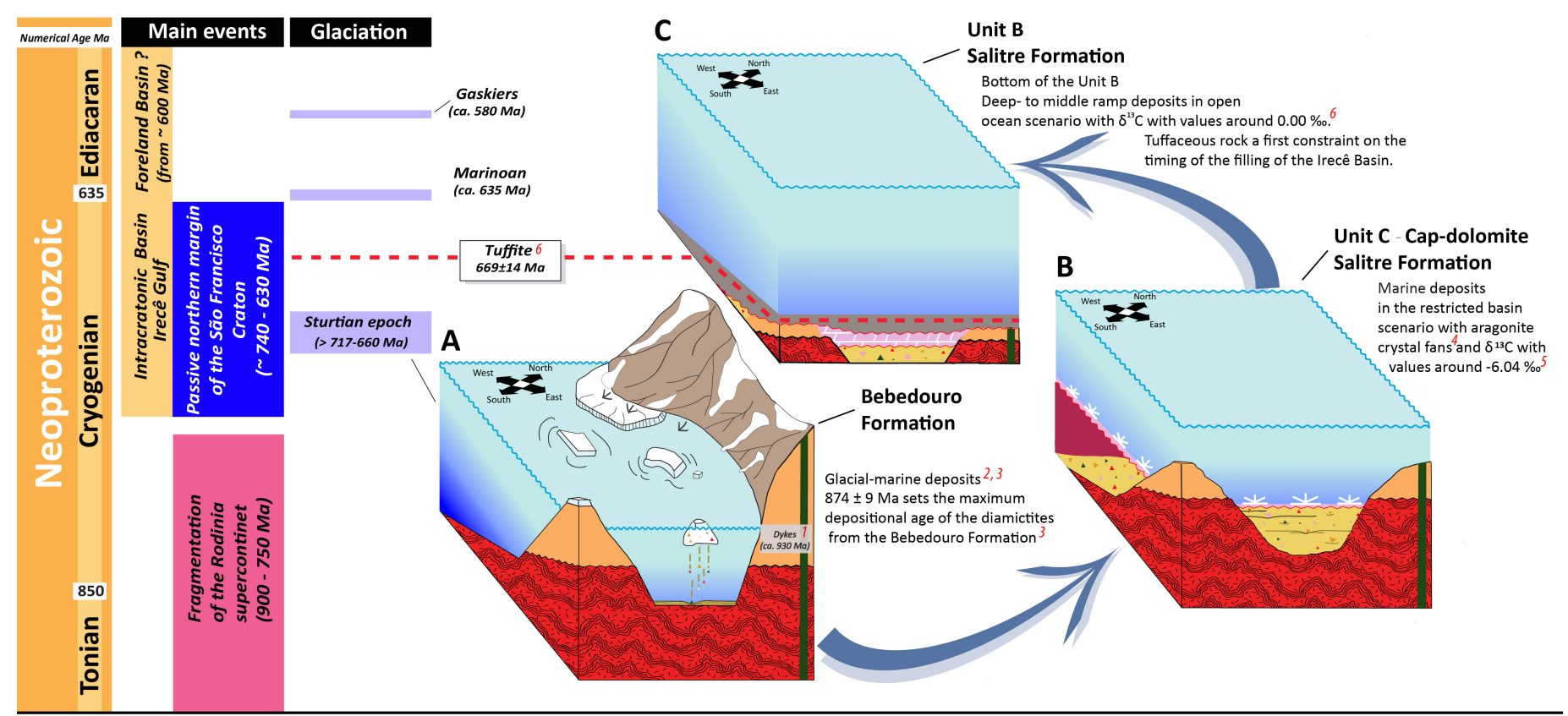

Key

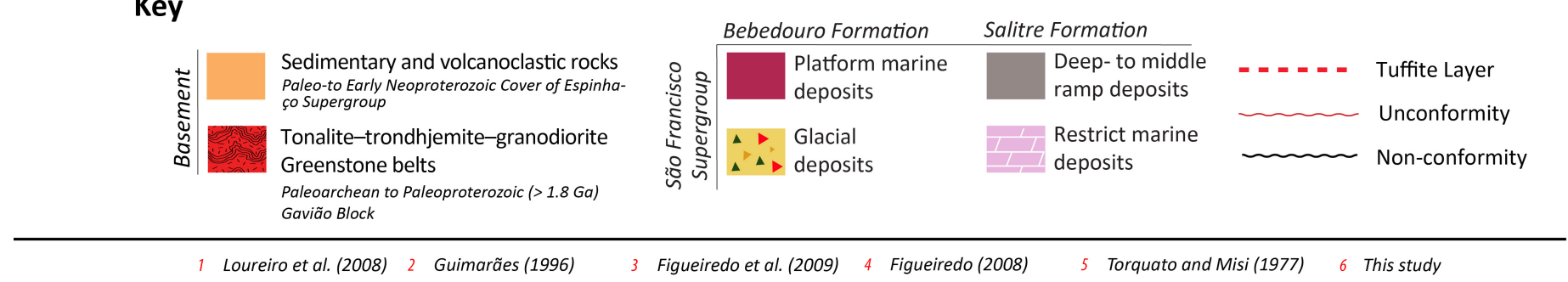

1 Loureiro et al. (2008) 2 Guimarães (1996) 3 Figueiredo et al. (2009) 4 Figueiredo (2008) 5 Torquato and Misi (1977) 6 This study

Figura 30. Schematic chart illustrating the evolution of the Irecê Basin from 720 Ma to > 580 Ma showing the A. rifting (glacial deposits of the Bebedouro Fm.) associated with

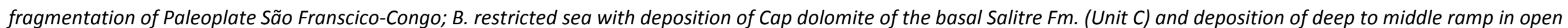
sea connected to the passive margin (Unit B). This sequence is covered by the foreland deposits (Unit A, A1, see figure 22), which it is not represented in this sketch (see text for explanation). 


\subsection{CONCLUSIONS}

The following conclusions can be outlined from stratigraphic, isotope chemostratigraphy and geochronological data, as follow:

- Facies analysis points to that the basal portion of the Unit B (Salitre Fm.) is a preserved carbonate marine sedimentation, probably deposited in a context of platform that prevailed ramp-type morphology.

- Slightly negative $\delta{ }^{13} \mathrm{C}$ values close to $0.0 \%$ support depositional marine environment after strong negative values of cap carbonates directly connected to underlying glacial deposits of the Bebedouro diamictites as part of Sturtian glacial epoch (Middle Cryogean).

- The identification of tuffite layer between the deposits of basal section of Unit B indicates volcanic activities. Geochemical data of this layer guided simply in High Field Strength Elements (HFSE) and the concentrations the rare earth element (REE) patterns plotted on specific diagrams suggests that tuffite may be formed after magma with a trachyandesitic composition. The REEs patterns are quite similar to the volcaniclastic greywackes of Precambrian. The tuffite displays a pattern quite similar to Cryogenian Alkaline Syenites REE composition. 
- In the study of provenance, we highlight precise and pioneer U-Pb zircon age (ca. $669 \mathrm{Ma}$ ) determination from tuffite layer, at volcanic (or magmatic; juvenile volcanic material) grain. The maximum depositional age of the Salitre Fm. is constrained by the age of the volcanic zircon grain at $669 \pm 14 \mathrm{Ma}$ for the basal units of the Unit B. This volcanism records are interpreted to be a later phase of initial rifting process either associated with alkaline intrusions in the basement close to the basin or any distal magmatism in the earlier stage of the passive margin.

- The analysis of the zircon age distribution pattern reveals the Gavião crustal block as the main source for sedimentary infilling of Irecê Gulf. However, a change in sedimentation pattern is recognized in the Unit B carbonate platform, where the basal portion of this unit contains Paleoarchean, Neo Archean and Rhyacian sources and the intermediate portion contain Archean and Paloeproterozoic sources and also subordinate Statherian and Calamnyan ones. The later sedimentation pattern is similar to the glacial underlying units, the Bebedouro Fm. or Caboclo and Upper Tombador Fm. of the Upper Espinhaço sequence, whereas basal section of the Unit B has zircon age contribution as those of Middle Espinhaço sequence (Lower Tombador and Açuruá Fm.).

- A gulf-like sedimentation is herein the best depositional model for the region where the sedimentation process started with the record of the Cryogenian rift stage (final phase of fragmentation of the Rodinia supercontinent) probable during the 
Sturtian Glacial period, represented by the basal diamictite and associated sedimentary rocks, the Bebedouro Formation, which was covered by the cap carbonates of Unit C. This sequence evolved to an intracratonic (gulf- like) connected to a passive margin (open ocean) sedimentation during Cryogenian period with deposition of Unit B in the Irecê Basin.

\section{Acknowledgments}

We thank Brazilian Research Agency - CNPq (grant 160391/2011-3) and PETROBRAS/Brazil for their financial support. This work benefits the comments and revision of Evandro Lima, Léo Rodrigues Teixeira and Luciano Alessandretti. We thank also thank Eduardo Mancini and Marcelo Soares for the stable isotope and geochemistry analyses. Ana Spohr, Andrei Vechini, Hemeli Ligabue, Henrique Lima, Hugo Vidal, Júlio Sanglard, Luan Krug, Marina Duarte, Ricardo Maahs, Tiago Morais and Viviane Araújo are thanked for assistance in collecting samples and data processing.

\section{Appendix A. Supplementary data}

Supplementary data associated with this article can be found in the online version. Consists of (1) Geospatial data kml of the study area; (2) Analytical data, and (3) Comparison of tuffite composition with composition of the Upper Continental Crust (Taylor and McLennan, 1985) and Proterozoic volcanics and sedimentary rocks (Condie, 1993). 


\section{References}

Alessandretti, L., Philipp, R.P., Chemale, F., Brückmann, M.P., Zvirtes, G., Matté, V., Ramos, V. A., 2013. Provenance, volcanic record, and tectonic setting of the Paleozoic Ventania Fold Belt and the Claromecó Foreland Basin: Implications on sedimentation and volcanism along the southwestern Gondwana margin. J. South Am. Earth Sci. 47, 12-31. doi:10.1016/j.jsames.2013.05.006

Alkmim, F.F., Marshak, S., Pedrosa-Soares, A.C., Peres, G.G., Cruz, S.C.P., Whittington, A., 2006. Kinematic evolution of the Araçuaí-West Congo orogen in Brazil and Africa: Nutcracker tectonics during the Neoproterozoic assembly of Gondwana. Precambrian Res. 149, 43-64. doi:10.1016/j.precamres.2006.06.007

Alkmim, F.F., Martins-Neto, M.A., 2012. Proterozoic first-order sedimentary sequences of the São Francisco craton, eastern Brazil. Mar. Pet. Geol. 33, 127-139. doi:10.1016/j.marpetgeo.2011.08.011

Alvarenga, C.J.S., Santos, R. V., Vieira, L.C., Lima, B.A.F., Mancini, L.H., 2014. MesoNeoproterozoic isotope stratigraphy on carbonates platforms in the Brasilia Belt of Brazil. Precambrian Res. 251, 164-180. doi:10.1016/j.precamres.2014.06.011

Amthor, J.E., Grotzinger, J.P., Schröder, S., Bowring, S.A., Ramezani, J., Martin, M.W., Matter, A., 2003. Extinction of Cloudina and Namacalathus at the Precambrian-Cambrian boundary in Oman. Geology 31, 431-434. doi:10.1130/00917613(2003)031<0431:EOCANA>2.0.CO;2

Araújo, C.E.G., Rubatto, D., Hermann, J., Cordani, U.G., Caby, R., Basei, M.A.S., 2014. Ediacaran 2,500-km-long synchronous deep continental subduction in the West Gondwana Orogen. Nat. Commun. 5, 5198. doi:10.1038/ncomms6198 
Arnaud, E., Halverson, G.P., Shields-Zhou, G., 2011. Chapter 1 The geological record of Neoproterozoic ice ages. Geol. Soc. London, Mem. 36, 1-16. doi:10.1144/M36.1

Babinski, M., Pedrosa-Soares, A.C., Trindade, R.I.F., Martins, M., Noce, C.M., Liu, D., 2012. Neoproterozoic glacial deposits from the Araçuaí orogen, Brazil: Age, provenance and correlations with the São Francisco craton and West Congo belt. Gondwana Res. 21, 451-465. doi:10.1016/j.gr.2011.04.008

Babinski, M., Vieira, L.C., Trindade, R.I.F., 2007. Direct dating of the Sete Lagoas cap carbonate (Bambuí Group, Brazil) and implications for the Neoproterozoic glacial events. Terra Nov. 19, 401-406. doi:10.1111/j.1365-3121.2007.00764.x

Bádenas, B., Aurell, M., 2001. Proximal-distal facies relationships and sedimentary processes in a storm dominated carbonate ramp (Kimmeridgian, Northwest of the Iberian Ranges, Spain. Sediment. Geol. 139, 319-340. doi:10.1016/S0037-0738(00)00151-2

Bahamonde, J.R., Kenter, J.A.M., Della Porta, G., Keim, L., Immenhauser, A., Reijmer, J.J.G., 2004. Lithofacies and depositional processes on a high, steep-margined Carboniferous (Bashkirian-Moscovian) carbonate platform slope, Sierra del Cuera, NW Spain. Sediment. Geol. 166, 145-156. doi:10.1016/j.sedgeo.2003.11.019

Barbosa, J.S.F., Sabaté, P., 2004. Archean and Paleoproterozoic crust of the São Francisco Craton, Bahia, Brazil: Geodynamic features. Precambrian Res. 133, 1-27. doi:10.1016/j.precamres.2004.03.001

Boynton, W.V., 1984. Cosmochemistry of the rare earth elements: meteorite studies. In: Henderson, P. (Ed.), Rare Earth Element Geochemistry. Elsevier, Amsterdam, 63-114. Brand, U., Veizer, J., 1980. Chemical Diagenesis of a Multicomponent Carbonate System-1: Trace Elements. J. Sediment. Petrol. Vol. 50, 1219 -1236. doi:10.1306/212F7BB7-2B24- 


\section{D7-8648000102C1865D}

Brito Neves, B.B.D., Costa Campos Neto, M., Fuck, R., 1999. From Rodinia to western Gondwana: An approach to the Brasiliano-Pan African cycle and orogenic collage. Episodes 22, 155-166.

Burchette, T.P., Wright, V.P., 1992. Carbonate ramp depositional systems. Sediment. Geol. 79, 3-57.

Burne, R.V., Moore, L.S., 1987. Microbialites: Organosedimentary deposits of benthic microbial communities. Palaios 2, 241-254.

Calvet, F., Tucker, M., 1988. Outer ramp cycles in the Upper Muschelkalk of the Catalan Basin, northeast Spain. Sediment. Geol. 57, 185-198.

Caxito, F.A., Uhlein, A., 2013. Arcabouço tectônico e estratigráfico da Faixa Riacho Do Pontal, divisa Pernambuc-Piauí-Bahia. Geonomos 21, 19-37.

Caxito, F.D.A., Dantas, E.L., Stevenson, R., Uhlein, A., 2014. Detrital zircon (U-Pb) and Sm-Nd isotope studies of the provenance and tectonic setting of basins related to collisional orogens: The case of the Rio Preto fold belt on the northwest São Francisco Craton margin, NE Brazil. Gondwana Res. 26, 741-754. doi:10.1016/j.gr.2013.07.007

Chaves, A.O., Menezes, C.B., Paula, S.C., 2014. Litoquímica dos diques máficos de Formiga/Pedro Lessa (Brasil) e Kinga-Comba/Sembé-Ouesso (África): marcadores da tafrogênese toniana no craton São Francisco-Congo. Brazilian J. Geol. 44, 05-11. doi:10.5327/Z2317-4889201400010002.

Chemale Jr., F., Alkmim, F.F., Endo, I., 1993. Late Proterozoic tectonism in the interior of theSão Francisco craton. In: Findlay, R.H., Unrug, R., Banks, M.R., Veevers, J.J. (Eds.), Gondwana Eight: Assembly, Evolution and Dispersal. Balkema, Rotterdam, 29-41. 
Chemale, F., Dussin, I.A., Alkmim, F.F., Martins, M.S., Queiroga, G., Armstrong, R., Santos, M.N., 2012. Unravelling a Proterozoic basin history through detrital zircon geochronology: The case of the Espinhaço Supergroup, Minas Gerais, Brazil. Gondwana Res. 22, 200-206. doi:10.1016/j.gr.2011.08.016

Condie, K.C., 1993. Chemical composition and evolution of the upper continental crust: contrasting results from surface samples and shales. Chemical Geology 104, 1-37.

Cruz, S.C.P., Alkmim, F.F., 2006. The tectonic interaction between the Paramirim Aulacogen and the Araçuaí Belt, São Francisco craton region, Eastern Brazil. An. Acad. Bras. Cienc. 78, 151-173. doi:/S0001-37652006000100014

Cruz, S.C.P., Barbosa, J.S.F., Pinto, M.S., Peucat, J.J., Paquette, J.L., Souza, J.S., Martins, V.S., Chemale Jr, F., Carneiro, M.A., 2016. The Siderian-Orosirian magmatism in the Gavião Paleoplate, Brazil: U-Pb geochronology, geochemistry and tectonic implications. J. South Am. Earth Sci. 69, 43-79. doi:10.1016/j.jsames.2016.02.007

Cruz, S.C.P., Peucat, J.J., Teixeira, L., Carneiro, M.A., Marques Martins, A.A., Santana, J.D.S., De Souza, J.S., Barbosa, J.S.F., Leal, A.B.M., Dantas, E., Pimentel, M., 2012. The Caraguataí syenitic suite, a ca. 2.7 Ga-old alkaline magmatism (petrology, geochemistry and U-Pb zircon ages). Southern Gavião block (São Francisco Craton), Brazil. J. South Am. Earth Sci. 37, 95-112. doi:10.1016/j.jsames.2011.11.006

Danderfer Filho, A., Lana, C.C., Nalini Júnior, H.A., Costa, A.F.O., 2014. Constraints on the Statherian evolution of the intraplate rifting in a Paleo-Mesoproterozoic paleocontinent: New stratigraphic and geochronology record from the eastern São Francisco craton. Gondwana Res. 28, 668-688. doi:10.1016/j.gr.2014.06.012

Dardenne, M.A., 1978. Síntese sobre a estratigrafia do Grupo Bambuí no Brasil Central, in: 
Congresso Brasileiro de Geologia. pp. 597-610.

Duarte, K.S., Vasconcellos, R.V.A., Rugenski, A., 2012. Contribuição da Geofísica na definição do potencial petrolífero da Bacia do Irecê. pp. 1-3.

Dunn, P.R., Thomson, B.P., Rankama, K., 1971. Late Pre-Cambrian Glaciation in Australia as a stratigraphic boundary. Nature 231, 498-502.doi: 10.1038/231498a0

Dussin, I.A., Chemale Jr., F., 2011. Geologia Estrutural e Estratigrafia do Sistema EspinhaçoChapada Diamantina e sua aplicação nas Bacias Mesocenozóicas da Margem Passiva Brasileira. Final Report.FUNDEP, Belo Horizonte (302 pp.).

Fanning, C.M., Link, P., 2006. Constraints on the timing of the Sturtian glaciation from southern Australia; ie for the true Sturtian. Abstr. with Programs - Geol. Soc. Am. 38, 115.

Fanning, C.M., Link, P.K., 2004. U-Pb SHRIMP ages of Neoproterozoic (Sturtian) glaciogenic Pocatello Formation, southeastern Idaho. Geology 32, 881-884. doi:10.1130/G20609.1 Figueiredo, F.T., 2008. Fácies sedimentares e proveniência da Formação Bebedouro, Neoproterozóico (BA). Master thesis, Universidade de São Paulo, Instituto de Geociências, 121 p.

Figueiredo, F.T., Almeida, R.P., Tohver, E., Babinski, M., Liu, D., Fanning, C.M., 2009. Neoproterozoic glacial dynamics revealed by provenance of diamictites of the Bebedouro Formation, São Francisco Craton, Central Eastern Brazil. Terra Nov. 21, 375385. doi:10.1111/j.1365-3121.2009.00893.x

Flügel, E., 2010. Microfacies of Carbonate Rocks: Analysis, Interpretation and Application. doi:10.1007/978-3-642-03796-2

Formoso, M.L.L.; Garcia, A.J.V.; Misuzaki, A.M.; Alves, D.B.; Calarge, L.M.; Gomes, M.E.B., 
1997. Permian Tonsteins from the Paraná Basin, Rio Grande do Sul, Brazil, in: Clays for Our Future - Proceedings of the 11th International Clay Conferenc., Ottawa, Canada, pp. $615-621$.

Frimmel, H.E., Klötzli, U.S., Siegfried, P.R., 1996. New Pb-Pb Single Zircon Age Constraints on the Timing of Neoproterozoic Glaciation and Continentaal Break-up in Namíbia. J. Geol. 104, 459-469.

Grotzinger, J., Al-Rawahi, Z., 2014. Depositional facies and platform architecture of microbialite-dominated carbonate reservoirs, Ediacaran-Cambrian Ara Group, Sultanate of Oman. Am. Assoc. Pet. Geol. Bull. 98, 1453-1494. doi:10.1306/02271412063

Guadagnin, F., Chemale, F., 2015. Detrital zircon record of the Paleoproterozoic to Mesoproterozoic cratonic basins in the São Francisco Craton. J. South Am. Earth Sci. 60, 104-116. doi:10.1016/j.jsames.2015.02.007

Guadagnin, F., Chemale, F., Magalhães, A.J.C., Santana, A., Dussin, I., Takehara, L., 2015a. Age constraints on crystal-tuff from the Espinhaço Supergroup - Insight into the Paleoproterozoic to Mesoproterozoic intracratonic basin cycles of the Congo-São Francisco Craton. Gondwana Res. 27, 363-376. doi:10.1016/j.gr.2013.10.009

Guadagnin, F., Chemale Junior, F., Magalhães, A.J.C., Alessandretti, L., Bállico, M.B., Jelinek, A.R., 2015b. Sedimentary petrology and detrital zircon U-Pb and Lu-Hf constraints of Mesoproterozoic intracratonic sequences in the Espinhaço Supergroup: Implications for the Archean and Proterozoic evolution of the São Francisco Craton. Precambrian Res. 266, 227-245. doi:10.1016/j.precamres.2015.05.027

Guimarães, J.T., 1996. A Formação Bebedouro no Estado da Bahia: Faciologia , Estratigrafia e Ambientes de Sedimentação. Universidade Federal da Bahia, Instituto de Geociências, 
$146 p$.

Guimarães, J. T.; Alkmim, F. F.; Cruz, S. C. P., 2012. In: Barbosa, J. S. (Coord.), Geologia da Bahia, Pesquisa e Atualização. CBPM, Série Publicações especiais, Salvador, Brazil, 3385.

Halverson, G.P., Hoffman, P.F., Schrag, D.P., Maloof, A.C., Rice, A.H.N., 2005. Toward a Neoproterozoic composite carbon-isotope record. Bull. Geol. Soc. Am. 117, 1181-1207. doi:10.1130/B25630.1

Halverson, G.P., Shields-Zhou, G., 2011. Chapter 4 Chemostratigraphy and the Neoproterozoic glaciations. Geol. Soc. London, Mem. 36, 51-66. doi:10.1144/M36.4

Halverson, G.P., Wade, B.P., Hurtgen, M.T., Barovich, K.M., 2010. Neoproterozoic $\begin{array}{llll}\text { chemostratigraphy. } & \text { Precambrian } & \text { Res. }\end{array}$ doi:10.1016/j.precamres.2010.04.007

Hoffman, P.F., 1998. A Neoproterozoic Snowball Earth. Science 281, 1342-1346. doi:10.1126/science.281.5381.1342

Hoffman, P.F., Schrag, D.P., 2002. The snowball Earth hypothesis: testing the limits of global change. Terra Nov. 14, 129-155. doi:10.1046/j.1365-3121.2002.00408.x

Hoffmann, K.H., Condon, D.J., Bowring, S.A., Crowley, J.L., 2004. U-Pb zircon date from the Neoproterozoic Ghaub Formation Namibia: Constraints on Marinoan glaciation. Geology 32, 817-820. doi:10.1130/G20519.1

Karfunkel, J., Hoppe, A., 1988. Late Proterozoic glaciation in central-eastern Brazil: Synthesis and model. Palaeogeogr. Palaeoclimatol. Palaeoecol. 65, 1-21. doi:10.1016/00310182(88)90108-3

Kaufman, A.J., Knoll, A.H., 1995. Neoproterozoic variations in the C-isotopic composition of 
seawater: stratigraphic and biogeochemical implications. Precambrian Res. 73, 27-49. doi:10.1016/0301-9268(94)00070-8

Keeley, J.A., Link, P.K., Fanning, C.M., Schmitz, M.D., 2012. Pre- to synglacial rift-related volcanism in the Neoproterozoic (Cryogenian) Pocatello Formation, SE Idaho: New SHRIMP and CA-ID-TIMS constraints. Lithosphere 5, 128-150. doi:10.1130/L226.1

Kennedy, M.J., 1996. Stratigraphy, sedimentology, and isotopic geochemistry of Australian Neoproterozoic Postglacial cap-dolostones: Deglaciation, $\mathrm{S}^{13} \mathrm{C}$ excursions, and carbonate precipitation. J. Sediment. Res. 66, 1050-1064.

Key, R.M., Liyungu, A.K., Njamu, F.M., Somwe, V., Banda, J., Mosley, P.N., Armstrong, R.A., 2001. The western arm of the Lufilian Arc in NW Zambia and its potential for copper mineralization. J. African Earth Sci. 33, 503-528. doi:10.1016/S0899-5362(01)00098-7

Knoll, A.H., Walter, M.R., Narbonne, G.M., Christie-blick, N., 2004. A New Period for the Geologic Time Scale. Science (80-. ) 305, 621-623.

Lagoeiro, L.E., 1990. Estudo da Deformação nas Sequências Carbonáticas do Grupo Una na região de Irecê, BA. Master thesis, Universidade Federal de Ouro Preto, Escola de Minas, $105 \mathrm{p}$.

Le Bas, M.J., Le Maitre, R.W., Streckeisen, A., Zanettin, B., 1986. A chemical classification of volcanic rocks on the total alkali-silica diagram. Journal of Petrology $27,745-750$.

Le Maitre, R.W. (Ed.), 2002. Igneous Rocks: A Classification and Glossary of Terms., Cambridge Univ Press. doi:10.1017/S0016756803388028

Loureiro, H.S.C., Lima, E.S., Macedo, E.R., Silveira, F.V., Bahiense, I.C., Arcanjo, J.B.A., Moraes Filho, J.C., Neves, J.P., Guimarães, J.T., Teixeira, L.R., Abram, M.B., Santos, R.A., Melo,R.C., 2008. Projeto Barra-Oliveira dos Brejinhos Geological map. Brazilian 
Geological Survey and Bahia Mineral Research Company, scale 1:200.000.

Ludwig, K.R., 2008. Manual for Isoplot 3.7: Berkeley Geochronology Center Special Publication No. 4.

Ludwig, K.R., 2003. Using Isoplot/Ex, version 3.00, A Geochronological Toolkit for Microsoft Excel. Berkeley Geochronology Center Special Publication No. 1.

Marshak, S., Alkmim, F.F., Whittington, A., Pedrosa-Soares, A.C., 2006. Extensional collapse in the Neoproterozoic Araçuaí orogen, eastern Brazil: A setting for reactivation of asymmetric crenulation cleavage. J. Struct. Geol. 28, 129-147. doi:10.1016/j.jsg.2005.09.006

McLennan, S.M., Hemming, S.,McDaniel, D.K., Hanson, G.N., 1993. Geochemical approaches to sedimentation, provenance, and tectonics. In: Johnsson,M.J., Basu, A. (Eds.), Processes Controlling the Composition of Clastic Sediments. Geol. Soc. of America Special Paper 284, 21-40.

Menezes, R.C.L., Conceição, H., Rosa, M.D.L.D.S., Macambira, M.J.B., Galarza, M.A., Rios, D.C., 2012. Geoquímica e geocronologia de granitos anorogênicos Tonianos (ca. 914-899 Ma) da Faixa Araçuaí no sul do Estado da Bahia. Geonomos 20, 1-13.

Misi, A., 1979. O Grupo Bambuí no Estado da Bahia. In: Inda, H.V. (Ed.), Geologia e Recursos Minerais do Estado da Bahia, Textos Básicos. Salvador, Brazil, 120-54.

Misi, A., Kaufman, A.J., Azmy, K., Dardenne, M.A., 2011. Neoproterozoic successions of the Sao Francisco Craton , Brazil: the Bambuí, Una, Vazante and Vaza Barris / Miaba groups and their glaciogenic deposits. Geol. Rec. Neoproterozoic Glaciat. 36, 509-522. doi:10.1144/M36.48

Misi, A., Kaufman, A.J., Veizer, J., Powis, K., Azmy, K., Boggiani, P.C., Gaucher, C., Teixeira, 
J.B.G., Sanches, A.L., Iyer, S.S.S., 2007. Chemostratigraphic correlation of Neoproterozoic successions in South America. Chem. Geol. 237, 161-185. doi:10.1016/j.chemgeo.2006.06.019

Misi, A., Kyle, J.R., 1994. Upper Proterozoic carbonate stratigraphy, diagenesis, and stromatolite phosphorite formation, Irece Basin, Bahia, Brazil. J. Sed. Res. A64, 299-310. Misi, A., Veizer, J., 1998. Neoproterozoic carbonate sequences of the Una Group, Irecê Basin, Brazil: chemostratigraphy, age and correlations. Precambrian Res. 89, 87-100. doi:10.1016/S0301-9268(97)00073-9

Misi, A., Souto, P.G., 1975. Controle estratigráfico das mineralizações de Pb-Zn-F-Ba do Grupo Bambui, parte leste da Chapada de Irecê (Bahia). Rev. Bras. Geociências, 5, 3045.

Narbonne, G.M., 2005. The Ediacara Biota: Neoproterozoic Origin of Animals and Their Ecosystems. Annu. Rev. Earth Planet. Sci. 33, 421-442. doi:10.1146/annurev.earth.33.092203.122519

Oliveira, E.P., Windley, B.F., Araújo, M.N.C., 2010. The Neoproterozoic Sergipano orogenic belt, NE Brazil: A complete plate tectonic cycle in western Gondwana. Precambrian Res. 181, 64-84. doi:10.1016/j.precamres.2010.05.014

Paula-Santos, G.M., Babinski, M., Kuchenbecker, M., Caetano-Filho, S., Trindade, R.I., Pedrosa-Soares, A.C., 2015. New evidence of an Ediacaran age for the Bambuí Group in southern São Francisco craton (eastern Brazil) from zircon U-Pb data and isotope chemostratigraphy. Gondwana Res. 28, 702-720. doi:10.1016/j.gr.2014.07.012

Pedrosa-Soares, A.C., Alkmim, F.F., 2011. How many rifting events preceded the development of the Araçuaí-West Congo orogen?.Geonomos 19, 244-251. 
Pedrosa-Soares, A.C., Alkmim, F.F., Tack, L., Noce, C.M., Babinski, M., Silva, L.C., MartinsNeto, M.A., 2008. Similarities and differences between the Brazilian and African counterparts of the Neoproterozoic Aracuai-West Congo orogen. Geol. Soc. London, Spec. Publ. 294, 153-172. doi:10.1144/SP294.9

Peucat, J.J., Mascarenhas, J.F., Barbosa, J.S.F., Souza, S.L., Marinho, M.M., Fanning, C.M., Leite, C.M.M., 2002. 3.3 Ga SHRIMP U-Pb zircon age of a felsic metavolcanic rock from the Mundo Novo greenstone belt in the São Francisco craton, Bahia (NE Brazil). J. South Am. Earth Sci. 15, 363-373. doi:10.1016/S0895-9811(02)00044-5

Pearce, J. A., Harris, N. B. W., Tindle, A. G., 1984. Trace element discrimination diagrams for the tectonic interpretation of granitic rocks. Journal of Petrology 25, 956-983.

Pimentel, M.M., Dardenne, M.A., Fuck, R.A., Viana, M.G., Junges, S.L., Fischel, D.P., Seer, H.J., Dantas, E.L., 2001. Nd isotopes and the provenance of detrital sediments of the Neoproterozoic Brasilia Belt, central Brazil. J. South Am. Earth Sci. 14, 571-585.

Pimentel, M.M., Fuck, R.A., Botelho, N.F., 1999. Granites and the geodynamic history of the Neoproterozoic Brasilia belt, Central Brazil: A review. Lithos 46, 463-483. doi:10.1016/S0024-4937(98)00078-4

Pimentel, M.M., Rodrigues, J.B., DellaGiustina, M.E.S., Junges, S., Matteini, M., Armstrong, R., 2011. The tectonic evolution of the Neoproterozoic Brasília Belt, central Brazil, based on SHRIMP and LA-ICPMS U-Pb sedimentary provenance data: A review. J. South Am. Earth Sci. 31, 345-357. doi:10.1016/j.jsames.2011.02.011

Renne, P.R., Onstott, T.C., D'Agrella-Filho, M.S., Pacca, I.G., Teixeira, W., 1990. 40Ar/39Ar dating of 1.0-1.1 Ga magnetizations from the São Francisco and Kalahari cratons: tectonic implications for Pan-African and Brasiliano mobile belts. Earth and Planetary 
Science Letters 101, 349-366. doi: 10.1016/0012-821X(90)90165-T

Reynolds, R.C., 1985. NEWMOD - A computer program for the calculation of onedimensional diffraction patterns of mixed-layered clays. 8 Brook Rd., Hanover, NH 03755

Riding, R., 2000. Microbial carbonates : the geological record of calcified bacterial-algal mats and biofilms. Sedimentology 47, 179-214. doi:10.1046/j.1365-3091.2000.00003.x

Rosa, M.L.S., Conceição, H., Macambira, M.J., Galarza, M.A., Cunha, M.P., Menezes, R.C.L., Marinho, M.M., Cruz Filho, B.E., Rios, D.C., 2007. Neoproterozoic anorogenic magmatism in the Southern Bahia Alkaline Province of NE Brazil: U-Pb and $\mathrm{Pb}-\mathrm{Pb}$ ages of the blue sodalite syenites. Lithos 97, 88-97. doi:10.1016/j.lithos.2006.12.011

Rosa, M.L.S., Conceição, H., Macambira, M.J.B., Marinho, M.M., Menezes, R.C.L., Cunha, M.P., Rios, D.C., 2005. Magmatismo Neoproterozóico no Sul do Estado da Bahia, Maciço Sienítico Serra das Araras: Geologia, Petrografia, Idade e Geoquímica. Rev. Bras. Geociências 35, 111-121.

Rooney, A.D., Strauss, J.V., Brandon, A.D., Macdonald, F.A., 2015. A Cryogenian chronology: Two long-lasting synchronous Neoproterozoic glaciations. Geology 43, 459-462. doi:10.1130/G36511.1

Roser, B.P., Korsch, R.J., 1988. Provenance signatures of sandstone-mudstone suites determined using discriminant function analysis of major-element data. Chemical Geology 67, 119-139.

Rosset, A., Min, A., Marques, L.S., Macambira, M.J.B., Ernesto, M., Renne, P.R., Piccirillo, E.M., 2007. Genesis and geodynamic significance of Mesoproterozoic and Early Cretaceous tholeiitic dyke swarms from the São Francisco craton (Brazil). J. South Am. 
Earth Sci. 24, 69-92. doi:10.1016/j.jsames.2007.02.002

Santos, T.C., Pimentel, M.M., Brito Neves, B.B., 2011. Proveniência de Sedimentos Detríticos da Porção Nordeste do Grupo Una/Bambuí, Chapada Diamantina-BA : Dados U-Pb SHRIMP, in: XIII Congresso Brasileiro de Geoquímica. 1263-1266.

Sato, K., Tassinari, C. C. G., Basei, M. A. S., Siga Jr, O., Onoe, A. T., Souza, M. D., 2014. Microssonda lônica de Alta Resolução e de Alta Sensibilidade (SHRIMP Ile/MC) do Instituto de Geociências da Universidade de São Paulo, Brasil: método analítico e primeiros resultados. Geol. USP, Sér. cient. 14, 3-18. doi:10.5327/Z1519$874 X 201400030001$.

Schmid, R., 1981. Descriptive nomenclature and classification of pyroclstic deposits and fragments: Recommendations of the IUGS Subcommission on the Systematics of Igneous Rocks. Geology 9, 41-43.

Silva, L.C., Pedrosa-Soares, A.C., Teixeira, L.R., Armstrong, R., 2008. Tonian rift-related, Atype continental plutonism in the Araçuaí Orogen, eastern Brazil: New evidence for the breakup stage of the São Francisco-Congo Paleocontinent. Gondwana Res. 13, 527-537. doi:10.1016/j.gr.2007.06.002

Silveira, E.M., Söderlund, U., Oliveira, E.P., Ernst, R.E., Leal, A.B.M., 2013. First precise U-Pb baddeleyite ages of 1500Ma mafic dykes from the São Francisco Craton, Brazil, and tectonic implications. Lithos 174, 144-156. doi:10.1016/j.lithos.2012.06.004

Simons, D.B., Richardson, E.V., Nordin Jr., C.F., 1965. Sedimentary structures generated by flow in alluvial channels. In: Middleton, G.V. (Ed.), Primary SedimentaryStructures and Their Hydrodynamic Interpretation., Soc. Sed. Geology, Spec. Publ., 34-52.

Taylor, S.R., McLennan, S.M., 1985. The continental crust: its composition and evolution. 
Blackwell, Oxford.

Teles, G., Chemale, F., de Oliveira, C.G., 2015. Paleoarchean record of the detrital pyritebearing, Jacobina Au-U deposits, Bahia, Brazil. Precambrian Res. 256, 289-313. doi:10.1016/j.precamres.2014.11.004

Terra, J.G.S., Spadini, A.R., França, A.B., Sombra, C.L., Zambonato, E.E., Juschaks, L.C.S., Arienti, L.M., Erthal, M.M., Blauth, M., Franco, M.P., Matsuda, N.S., Goulart, N., Moretti Junior, P.A., D’Ávila, R.S.F., Souza, R.S., Tonietto, S.N., Anjos, S.M.C., Campinho, V.S., Winter, W.R., 2010. Classificação de rochas carbonáticas aplicável às bacias sedimentares brasileiras. B. Geoci. Petrobras, Rio Janeiro 18, 9-29.

Thompson, R.N., 1982. Magmatism of the British Tertiary volcanic province. Scottish Journal of Geology, 18, 49-107.

Thompson, D., Rainbird, R.H., Dix, G., 2014. Architecture of a neoproterozoic intracratonic carbonate ramp succession: Wynniatt Formation, Amundsen Basin, Arctic Canada. Sediment. Geol. 299, 119-138. doi:10.1016/j.sedgeo.2013.11.005

Torquato, J.R., Misi, A., 1977. Medidas Isotópicas de Carbono e Oxigênio em Carbonatos do Grupo Bambuí, na Região Centro-Norte do Estado da Bahia. Rev. Bras. Geociências 7, $14-24$.

Toulkeridis, T., Babinski, M., Buchwaldt, R., Brito Neves, B. B., Todt, W., Santos, R. 1999. Are Varangian or Sturtian the glacial deposits on the Sao Francisco Craton? Evidence from age determination of sedimentary rocks and minerals of the Neoproterozoic Una Group. II South American Symposium on Isotope Geology, Cordoba, Ext. Abstr., 453-456.

Trompette, R., Uhlein, A., Silva, M.E., Karmann, I., 1992. The Brasiliano São Francisco craton revisited (central Brazil). J. South Am. Earth Sci. 6, 49-57. doi:10.1016/0895- 
9811(92)90016-R

Verkouteren, R.M., Klinedinst, D.B., 2004. NIST Special Publication 260-149 2004 Edition.

Vicat, J.P., Pouclet, A., 2000. Palaeo- and Neoproterozoic granitoids and rhyolites from the West Congolian Belt (Gabon, Congo, Cabinda, north Angola): Chemical composition and geotectonic implications. J. African Earth Sci. 31, 597-617. doi:10.1016/S0899$5362(00) 80009-3$

Vieira, L.C., Nédélec, A., Fabre, S., Trindade, R.I.F., Almeida, R.P., 2015. Aragonite crystal fans in Neoproterozoic Cap Carbonates: A case study from Brazil and implications for the Post-snowball earth coastal environment 285-300. doi:10.2110/jsr.2015.21

Vieira, L.C., Trindade, R.I.F, Nogueira, A.C.R., Ader, M., 2007. Identification of a Sturtian cap carbonate in the Neoproterozoic Sete Lagoas carbonate plataform, Bambuí Group, Brazil. C. R.Geoscience 339, 240-258.

Warren, L. V, Quaglio, F., Riccomini, C., Simões, M.G., Poiré, D.G., Strikis, N.M., Anelli, L.E., Strikis, P.C., 2014. The puzzle assembled: Ediacaran guide fossil Cloudina reveals an old proto-Gondwana seaway. Geology 42, 391-394. doi:10.1130/G35304.1\r10.1130/00917613(2003)0310431:EOCANA2.0.CO;2;

Wedepohl, K.H., 1995. The composition of the continental crust. Geochimica et Cosmochimica Acta 59,217-1־-239. doi: 10.1016/0016-7037(95)00038-2.

Winchester, J.A., Floyd, P.A., 1977. Geochemical discrimination of different magma series and their differentiation products using immobile elements. Chem. Geol. 20, 325-343. doi:10.1016/0009-2541(77)90057-2

Zhou, C., Tucker, R., Xiao, S., Peng, Z., Yuan, X., Chen, Z., 2004. New constraints on the ages of Neoproterozoic glaciations in south China. Geology 32, 437-440. doi:10.1130/G20286.1 


\section{CONCLUSÕES}

- Um arcabouço de estratigrafia de sequências em alta resolução é proposto para o trecho superior da Unidade B, sul do sinclinal de Irecê, na bacia homônima. O trabalho de detalhe possibilitou caracterizar dez fácies e oito subfácies. A sucessão vertical de fácies mostra diferentes associações faciológicas: rampa intermediária com influência microbial (FA1); rampa interna rasa/rampa intermediária proximal (FA2); rampa interna dominada por microbialitos (FA3); rampa subaérea/sabkha (FA4). Estas FAs compõem um típico sistema carbonático marinho desenvolvido em uma rampa homoclinal.

- A análise sequencial em alta resolução distinguiu sequências elementares e de pequena escala em intervalos com a predominância das FA3 e FA4. Sequências elementares possuem espessura de algumas dezenas de centímetros. Estas sequências foram delineadas em campo a partir de uma série de fatores ordenados que pela sucessão vertical demonstrasse uma periodicidade/variabilidade no tipo do crescimento microbial.

- Sequências de pequena escala são constituídas a partir das sequências elementares. Sua demarcação ocorre a partir de critérios sedimentológicos e com o auxílio de perfis gama espectral. Sequências de média escala foram delineadas a partir de mudanças paleoambientais, como mudanças de associações 
faciológicas. Uma sequência de ampla escala, dezenas de metros de espessura, sugere a mais significativa mudança nas condições ambientais.

- A porção basal a intermediária da Unidade B foi investigada com foco em sedimentologia e proveniência de sedimentar, com análises isotópicas de $\mathrm{C}$ e $\mathrm{O}$ e $\mathrm{U} / \mathrm{Pb}$ SHRIMP. Valores pouco negativos de $\delta^{13} \mathrm{C}$, próximos de $0,0 \%$, sugerem uma assinatura isotópica típica de oceano. As análises $\mathrm{U} / \mathrm{Pb}$ foram realizadas em 2 amostras, sendo uma de siltito tufáceo - fácies FI (v) - e outra de calcário com níveis pelíticos. Uma abordagem geoquímica preliminar do nível com contribuição vulcânica indicou uma composição traquiandesítica e assinatura de Elementos Terra Raras compatível com a de grauvacas vulcanoclásticas do PréCambriano (Condie, 1993).

- A análise dos padrões de proveniência revelou a existência de 2 intervalos, com distintas assinaturas na distribuição de zircões. O primeiro foi identificado na amostra PRP-16, proveniente da base da Unidade B e coletada na Pedreira Rio Preto, e o segundo está representado na amostra selecionada na porção intermediária da unidade estudada, PEC-1, coletada na escarpa contígua ao Povoado Bom Jesus da Pecuária.

- Na base da Unidade B predominam idades arqueanas (3,38 - 2,56 Ga) e paleoproterozoicas (2,07 Ga). A idade mais nova obtida é de $669 \pm 14 \mathrm{Ma}$. A amostra PEC-1 apresenta um padrão diferente, e os grãos têm idade arqueana (3.29, 2,89 e 2,63 Ga), Paleoproterozoica (2,10 Ga), Estateriana (1,78 Ga) e 
Calaminiana (1,50 Ga). No estudo sobre proveniência, destaca-se a idade de cerca de 670 Ma obtida na camada de tufito. A idade de deposição máxima da Unidade B é limitada por esta determinação, cuja fonte pode estar vinculada ao magmatismo distal em ambiente tectônico de margem passiva, que à época se processava a NNE, na faixa Riacho do Pontal, ou ao sul, na Faixa Araçuaí, ou ainda, ao processo extensional de grande escala, bem documentado na contraparte africana da paleoplaca São Francisco-Congo, o vulcanismo La Loiula. Alternativamente, este magmatismo poderia estar relacionado com as atividades orogenéticas da Faixa Brasília.

- Especula-se que as demais fontes de sedimento são locais, como rochas granito-gnáissicas e greesntone belts associados do Bloco Gavião (>aprox. 2,0 Ga), embasamento do CSF na área de estudo, e rochas associadas aos vulcanismos Rio dos Remédios, Bomba e Tombador (aprox. 1,75 Ga, 1,5 e 1,38 Ga, respectivamente). Registros para inferência de fontes secundárias foram reportados apenas na amostra PEC-1.

- O estudo de proveniência, aliado à análise estratigráfica, sugere que durante a deposição da Unidade $B$, a Bacia de Irecê possuía uma configuração condizente com uma bacia sag, intracratônica, possivelmente tratando-se de um golfo associado ao desenvolvimento da margem passiva que se implantou nas faixas Riacho do Pontal e Sergipana, localizadas a NNE. 


\section{REFERÊNCIAS}

AITKEN, J.F.; HOWELL, J. High resolution sequence stratigraphy: innovations, applications and future prospects. In: HOWELL, J.A.; AITKEN, J.F. (Ed). High resolution sequence stratigraphy: Innovations and applications. London: Geological Society (Geological Society special publication, 104), 1996. p. 1-9.

ALKMIM, F.F. O que faz de um cráton um cráton? O Cráton do São Francisco e as revelações almeidianas ao delimita-lo. In: MANTESSO-NETO V.; BARTORELLI A.; CARNEIRO C.D.R.; BRITONEVES B.B. (Orgs.) Geologia do Continente Sul-Americano: evolução da obra de Fernando Flávio Marques de Almeida. São Paulo: Beca, 2004. p.17-35.

ALKMIM, F.F.; MARTINS-NETO, M.A. Proterozoic first-order sedimentary sequences of the Sãoo Francisco craton, Eastern Brazil. Mar. Petrol. Geol., v. 33, 2012. p.127-139.

ALKMIM F.F.; NEVES, B.B.B.; ALVES, J.A.C. Arcabouço Tectônico do Cráton do São Francisco Uma Revisão. O Cráton do São Francisco, II Simpósio sobre o Cráton do São Francisco, Salvador, Bahia, 1993.

ALMEIDA, F.F.M. Origem e evolução da plataforma brasileira. Boletim da Divisão de Geologia e Mineralogia, Departamento Nacional de Produção Mineral (DNPM), Ministério de Minas e Energia, Rio de Janeiro, v. 241, 36 p. 1967. 
ALMEIDA, F.F. O Craton do Sao Francisco. Revista Brasileira Geociências, v. 4, p. 349-364. 1977.

ALVARENGA, C.J.S.; SANTOS, R.V.; DANTAS, E.L. C-O-Sr isotopic stratigraphy of cap carbonates overlying Marinoan-age glacial diamictites in the Paraguay Belt, Brazil. Precambrian Research, v. 13, , 2004. p.1-21.

BABINSKI, M.; PEDREIRA, A.J.; BRITO NEVES, B.B.; VAN SCHMUS, W.R. Contribuição à geocronologia da Chapada Diamantina. In: Pedreira, A.J. (Ed.), VII Simpósio Nacional de Estudos Tectônicos. Brazilian Geological Society, 1999. p.118-120.

BARBOSA, J.S.F.; SABATÉ, P. Archean and Paleoproterozoic crust of the São Francisco Craton, Bahia, Brazil: geodynamic features. Precambrian Res., v. 133, 2005. p. 1-27.

BASTOS LEAL, L.R.; CUNHA, J.C.; CORDANI, U.G.; TEIXEIRA, W.; NUTMAN, A.P.; MENEZES LEAL, A.B.; MACAMBIRA, M.J.B. SHRIMP U-Pb, 207Pb/206Pb zircon dating and Nd isotopic signature of the Umburanas greenstone belt, Northern São Francisco Craton, Brazil. J. of South American Earth Sciences, 15, 2003. p.775-785.

BENTO FREIRE, E.; TERRA, G.J.; RAJA GABAGLIA, G.; LYKAWKA, R.; RODRIGUES, E.B. Searching for potential analogues for the Pre-Salt Santos Basin, Brazil: High Resolution Stratigraphic studies of microbialite-bearing sucessions from Salta Basin, Argentina. In: AAPG International Conference and Geologists, 2011. p. 35. 
BENTO FREIRE, E. Caracterização estratigráfica em alta resolução das sequências calcárias de origem microbiana do intervalo paleocênico da Formação Yacoraite (Sequência Balbuena IV) na região de Salta - Argentina. 2012. 243 p. Mestrado em Geologia Programa de Pós-graduação em Geologia, Instituto de Geociências, Universidade Federal do Rio de Janeiro, Rio de Janeiro, 2012.

BIZZI, L.A.; SCHOBBENHAUS, C.; VIDOTTI, R.M.; GONÇALVES, J.H. (Eds.) Geologia, Tectônica e Recursos Minerais do Brasil - Texto, Mapas e SIG. CPRM - Serviço Geológico do Brasil, Brasília, 674 p., 2003.

BOMFIM, L.F.C.; ROCHA, A.J.D.; PEDREIRA, A.J.; MORAIS, J.C., P; GUIMARES, J.T.; TESCH, N.A. Projeto Bacia de Irecê. Salvador, CPRM. (Relatório Final), 1985.

BRANCO , J.J.R.; COSTA, M.T. 1961. Roteiro para a excursão Belo Horizonte - Brasília. XV Congresso de Geologia. Escola de Engenharia da Universidade de Minas Gerais. Belo Horizonte, v. 15, 25 p.

BRANNER, J.C. Aggraded limestone plains of the interior of Bahia and climatic changes suggested by them. Geological Society of America Bulletin, v. 22, n. 2, 1911. p. 187-206.

BRITO NEVES, B.B.; CAMPOS NETO, M.C.; FUCK, R.A. From Rodinia to Western Gondwana: an approach to the Brasiliano-Pan African Cycle and orogenic collage. Episodes, Ottawa, v. 22, n. 3, 1999. p. $155-166$. 
BROWN, L.F.; FISHER W.L. Seismic-stratigraphic interpretation of depositional systems: Examples from Brazilian rift and pull-apart basins. In: PAYTON, C.E. (Ed.) Seismic Stratigraphy-applications, 1977. p. 213-248.

BURNE, R.V.; MOORE, L.S. Microbialites: organossedimentary deposits of benthic microbial communities. Palaios, v .2, 1987. p. 241-254.

CATUNEANU, O. Principles of Sequence Stratigraphy. 1 ed. Amsterdam: Elsevier, 2006, v. 1, $375 \mathrm{p}$.

CATUNEANU, O. et al. Towards the standardization of sequence stratigraphy.

Earth-Science Reviews, Elsevier, v. 92, ed.1-2, p. 1-33, 2009.

CATUNEANU, O. et al. Sequence Stratigraphy: Methodology and Nomenclature. Newsletters on Stratigraphy, Stuttgart, v. 44, n. 3, p.173-245, 2012.

CHEMALE JR. F.; DUSSIN, I.A.; ALKMIM, F.F.; MARTINS, M.S.; QUEIROGA, G.; ARMSTRONG, R.; SANTOS, M.N. Unravelling a Proterozoic basin history through detrital zircon geochronology: the case of the Espinhaço Supergroup, Minas Gerais, Brazil. Gondwana Res., v. 22, p. 200206, 2012.

CHOQUETTE, P.W.; PRAY, L.C. Geologic nomenclature and classification of porosity in sedimentary carbonates. AAPG Bulletin, v. 54, n. 2, p. 207-244, 1970. 
COHEN, K.M.; FINNEY, S.C.; GIBBARD, P.L.; FAN, J.X. The ICS International Chronostratigraphic Chart. Episodes, v. 36, p. 199-204, 2013.

CRUZ, S.C.P. A interação tectônica entre o Aulacógeno do Paramirim e o Orógeno AraçuaíOeste Congo. 2004. 503 p. Tese de Doutorado, Departamento de Geologia, Universidade Federal de Ouro Preto, 2004.

CRUZ, S.C.P.; ALKMIM, F.F. The tectonic interaction between the Paramirim Aulacogen and the Araçuaí Belt, São Francisco Craton region, Easter Brazil. Anais da Academia Brasileira de Ciências, v. 78, n. 1, p. 151-174, 2006.

DANDERFER, A. Análise estrutural descritiva e cinemática do Supergrupo Espinhaço na região da Chapada Diamantina (BA). 1990. 99 p. Dissertação de Mestrado, Departamento de Geologia, Universidade Federal de Ouro Preto, 1990.

DANDERFER, A. Geologia sedimentar e evolução tectônica do Espinhaço Setentrional, Estado da Bahia. 2000. 494 p. Tese de Doutoramento, Instituto de Geociências, Universidade de Brasília, 2000.

DARDENNE, M.A. Síntese sobre a estratigrafia do Grupo Bambuí no Brasil Central. In: Congresso Bras. Geologia, 31, Soc. Bras. Geologia, Recife, Anais..., v. 2, p. 597-610, 1978.

DAVIES, S.J.; ELLIOTT, T. Spectral Gamma ray characterization of high resolution sequence stratigraphy: examples from Upper Carboniferous fluvio-deltaic systems, County Clare, 
Ireland. In: HOWELL, J.A.; AITKEN, J. F. (Ed). High resolution sequence stratigraphy: Innovations and applications. London: Geological Society, (Geological Society special publication, 104), 1996. p. 25-35.

DERBY, O.A. Contribuição para o estudo da geologia do Vale do Rio São Francisco. Arquivos do Museu Nacional, v. 4, 1880.

DERBY, O.A. Notas geológicas sobre o Estado da Bahia. Sec. Agr. Via Ind. e O. Púb. Bol. Ano III, v. VII, n 1-3, p. $13-31,1905$.

DERBY, O.A. The Serra do Espinhaço, Brazil. Journal of Geology. v. 14, p. 374-401, 1906.

DOMINGUEZ, J.M.L.; ROCHA, A.J.D. Grupos Chapada Diamantina (Proterozóico Médio) e Una (Proterozóico Superior). In: O Proterozóico Médio e superior no Brasil: sedimentologia e evolução estrutural - conferência de campo, Morro do Chapéu. Roteiro de Excursão Geológica. Salvador: CPRM, 1991. 42p.

DOMINGUEZ, J.M.L. As coberturas do Cráton do São Francisco: uma abordagem do ponto de vista da análise de bacias. Em: DOMINGUEZ, J.M.L.; MISI, A. (Eds). O Cráton do São Francisco. Salvador. SBG, 1993. p. 137-159.

DUNHAM, R.J. Classification of carbonate rocks according to depositional texture. In: HAM, W.E. (Ed.) Classification of carbonate rocks. Tulsa: American Association of Petroleum Geologists, 1962, Memoir 1. p. 108-122. 
EMBRAPA. Embrapa Monitoramento por Satélite, 2011. Disponível em: <http://www.relevobr.cnpm.embrapa.br>. Acessado em 15 abril de 2011.

EMBRY, A.F.; KLOVAN, J.E. A Late Devonian Reef Tract on Northeastern Banks Island, Northwest Territories. Bulletin of Canadian Petroleum Geology, v. 19, n. 4, p. 730-781, 1971.

ESRI. ArcGIS, 2010. Disponível em: <http://www.esri.com/software/arcgis/index.html>. Acessado em 12 abril 2010.

FIGUEIREDO, F.T. Fácies sedimentares e proveniência da Formação Bebedouro, Neoproterozóico (BA). 2008. 121 p. Dissertação de Mestrado, Instituto de Geociências, Universidade de São Paulo, São Paulo, 2008.

FLINT, A.R. The Geology and Mineralization of the Irecê region. North-East Brazil 1979. 165 p. B.Sc. dissertation, University of London, London, 1979.

FLÜGEL, E. Microfacies of carbonate rocks: analysis, interpretation and application. Berlim: Springer, 2010.976p.

FOLK, R.L. Practical petrographic classification of limestones. Bull. Amer. Assoc. Petrol. Geol. v. $43 / 1$, p.1-38, 1959. 
FOLK, R.L. Petrology of Sedimentary Rocks. Austin: Hemphill, 1980. 182 p.

FOLK, R.L. Spectral Subdivision of Limestone Types. In: HAM, W.E. (Ed.), Classification of Carbonate Rocks. Tulsa: American Association of Petroleum Geologists, Memoir 1, 1962. p. 62-85.

FOLK, R.L. Detection of organic matter in thin sections of carbonate rocks using a white card. Sedimentary Geology, v. 54, p. 193-200, 1987.

FRIMMEL, H.E. Trace element distribution in Neoproterozoic carbonates as palaeoenvironmental indicator. Chemical Geology, v. 258, p. 338-353, 2009.

GUADAGNIN, F.; CHEMALE JR. F.; MAGALHÃES, A. J.; SANTANA, A.; DUSSIN, I.A.; TAKEHARA, L. Age constrains on crystal-tuff from the Espinhaço Supergroup - Insight into the Paleoproterozoic to Mesoproterozoic intracratonic basin cycles of the São Francisco Craton. Gondwana Res. v. 27, p. 363-376, 2015.

GUIMARÃES, J.T. A Formação Bebedouro no estado da Bahia: faciologia, estratigrafia e ambientes de sedimentação. 1996. 155f. Dissertação (Mestrado em Geociências) - Instituto de Geociências, Curso de Pós-Graduação em Geociências, Universidade Federal da Bahia, Bahia, 1996.

GUIMARÃES, J.T. Projeto Ibitiara - Rio de Contas. Salvador: CPRM \& CBPM, p. 182, 2005. 
GUIMARÃES, J.T.; SANTOS, R.A.; MELO, R.C. Geologia da Chapada Diamantina Ocidental (Projeto Ibitiara-Rio de Contas). Salvador: CPRM \& CBPM, 2008, Série Arquivos Abertos, 2008, v. 31, 68 p.

GUIMARÃES, J.T.; ALKMIM, F.F.; CRUZ, S.C.P. In: BARBOSA, J.S. (Coord.) Geologia da Bahia, Pesquisa e Atualização, Salvador: CBPM, Série Publicações especiais, v. 13, 2012. p. 33- 85.

HALDAR, S.K. Introduction to Mineralogy and Petrology. Elsevier, 2013. 338 p.

HALL, S.; STERNER, M.; SHUKLA, R. Application of cuttings gas/oil analysis, rapid XRF and high-resolution photography to reservoir evaluation. World Oil, April 2013 issue, p. 163-168, 2013.

HALVERSON, G.P.; WADW, B.P.; HURTGENC, M.T.; BAROVICH, K.M. Neoproterozoic chemostratigraphy. Precambrian Research, v. 182, p. 337- 350, 2010.

HARTT, C.F. Geology and Physical Geography of Brazil. Fields Osgood \& Co., Boston, 1870. p.620.

HERRON, M.M.; MATTERSON, A. Elemental composition and nuclear parameters of some common sedimentary minerals. Nuclear Geophysics, v. 7, p. 383-406, 1993.

HURST, A. Natural gamma ray spectrometry in hydrocarbon-bearing sandstones from the Norwegian Continental Shelf. In: HURST, A.; LOVELL, M. A.; MORTON, A. C. (Ed) Geological Applications of Wireline Logs. London: Geological Society, 1990. p. 211-222. 
HOFFMAN, P.F. On Cryogenian (Neoproterozoic) ice-sheet dynamics and the limitations of the glacial sedimentary record. South African Journal Of Geology, v. 108, p. 557-576, 2005.

INDA, H.A.V.; BARBOSA, J.F. Texto explicativo para o mapa geológico do estado da Bahia. Escala 1:1.000.000. Salvador: CPRM, 1978, 137 p.

INPE. Imagem de Satélite. Brasília: Instituto Nacional de Pesquisas Espaciais, 2010. Disponível em: <http://www.inpe.gov.br>. Acessado em 12 janeiro de 2010.

JPL - Jet Propulsion Laboratory. SRTM - The Mission to Map the World, California Inst. of Techn. Disponível em: <http://www2.jpl.nasa.gov/srtm/>. Acessado em 10 janeiro de 2011.

KARAGODIN, Yu.N. Relações mútuas entre os complexos cíclicos sedimentares em seções de bacias contendo petróleo e gás. Dohl. Akad. Nauk. SSSR, n. 220, p. 1414-1416 (traduzido do russo por FUCS, A.), 1975.

KYLE, J.R.; MISI, A. Origin of Zn-Pb-Ag sulfide deposits within Upper Proterozoic phosphaterich carbonate strata, Irecê Basin, Bahia, Brazil. Intern. Geol. Rev., 39:383-399, 1997.

LE MAITRE, R.W. (Ed.) Igneous rocks A Classification and Glossary of Terms, Recommendations of the International Union of Geological Sciences Subcommission on the Systematics of Igneous Rocks. Cambridge University Press, Cambridge, 2002. p. 9. 
LOVLEY, D.R.; PHILIPS, E.J.P.; GORBY, Y.A.; LANDA, E.R. Microbial reduction of uranium. Nature, v. 350, p. 413-416, 1991.

LOUREIRO, H.S.C. et al. Projeto Barra e Oliveira dos Brejinhos. Salvador, Companhia de Pesquisa de Recursos Minerais (CPRM) 283 p, 2008.

LUCIA, F.J. Carbonate reservoir characterization. 2nd edition: New York: Springer-Verlag, 2007. 226 p.

LUDWIG, K.R., 2008, Manual for Isoplot 3.7: Berkeley Geochronology Center, Special Publication No. 4. rev. August 26, 2008, 77 p.

LUDWIG, K.R., 2003. Using Isoplot/Ex, Version 3.00, a Geochronological Toolkit for Microsoft Excel. Berkeley Geochronology Center (Special Publication No. 1).

MACKEY, E.A.; CHRISTOPHER, S.J.; LINDSTROM, R.M.; LONG, S.E.; MARLOW, A.F.; MURPHY, K.E.; ... NEBELSICK, J. Certification of Three NIST Renewal Soil Standard Reference Materials for Element Content: SRM 2709a San Joaquin Soil, SRM 2710a Montana Soil I, and SRM 2711a Montana Soil II. National Institute of Standards and Technology Special Publication, p. 0-39, 2010.

MARINHO, M.M. La Séquence Volcano-Sedimentaire de Contendas-Mirante et la Bordure Occidentale du Bloc Jequié (Cráton du São Francisco-Brésil): Un exemple de Transition 
Archéean-Protérozoique. 1991. 388 p. Ph.D. Thesis. Blaise Pascal University, Clermont Ferrand, França, 1991.

MCLENNAN, S.M.; BOCK, B.; HEMMING, S.R.; HUROWITZ, J.A.; LEV, S.M.; MCDANIEL, D.K. The role of provenance and sedimentary processes in the geochemistry of sedimentary rocks. In: D.R.Lentz (ed.) Geochemistry of Sediments and Sedimentary Rocks: Evolutionary Considerations to Mineral Deposit-Forming. Geological Association of Canada, GeoText4, 2003, p. 7-38.

MENDELEY. MANUAL Mendeley - english. Mendeley Desktop, 1-16. 2011. Disponível em: <http://www.mendeley.com>. Acessado em: 05 março de 2013.

MIALL, A.D. A Review of the Braided-River Depositional Environment. Earth-Science Reviews, v. 13, n. 1, p. 1-62, 1977.

MISI, A. 1973 As mineralizações de chumbo-zinco-fluor-bário do vale do rio Jacaré (BA) e seu aspecto geológico. Tese apresentada à UFBA. Professor Assistente.

MISI, A. 1978 Ciclos de sedimentação e mineralizações de chumbo-zinco nas sequências Bambuí (Supergrupo São Francisco), Estado da Bahia. In: Simpósio de Mineralizações Sedimentológicas, Anais XXX Congresso Brasileiro Geol Recife, v. 6, p. 2548-2561.

MISI, A. O Grupo Bambuí no Estado da Bahia. In: INDA, H.A.V. (Ed.). Geologia

e Recursos Minerais do Estado da Bahia: Textos básicos. Salvador: COM, 1979. v. 1, p. 119154. 
MISI. A.; KAUFMAN, A.J.; VEIZER, J.; POWIS, K.; AZMY, K.; BOGGIANNI, P.C.; GAUCHER, C.;TEIXEIRA, J.B.G.; SANCHES, A.L.; IYER, S.S S., 2007. Chemostratigraphic Correlation of Neoproterozoic Successions in South America. Chem. Geol., 237, 161-185.

MISI, A.; KYLE, J.R. Upper Proterozoic carbonate stratigraphy, diagenesis, and stromatolitic phosphorite formation, Irecê Basin, Bahia, Brazil: Jour. Sed. Research., v. A, 64, p. 299-310, 1994.

MISI, A.; SOUTO, P.G. Controle estratigráfico das mineralizações de Pb-Zn-F-Ba do Grupo Bambui, parte leste da Chapada de Irecê (Bahia). Rev. Bras. Geociências, v. 5, p. 30-45, 1975. MISI, A.; SILVA, M.G. Chapada Diamantina Oriental, Bahia. Geologia e Depósitos Minerais. Superintendência de Geologia e Recursos Minerais/Universidade Federal da Bahia, Salvador, 194 p. 1996.

MISI, A.; VEIZER, J. Chemostratigraphy of Neoproterozoic Carbonate Sequences of the Una Group, Irecê Basin, Brazil. In: CONGRESSO BRASILEIRO DE GEOLOGIA, 39, Salvador, 1996, Anais. Salvador, Soc. Bras. Geol., 1996. v. 5, p. 487-489.

MONTEIRO, M.D.; SILVA, A.B. DA; CARVALHO, M.P. DE; FRÓS, R.J.B.; SOUZA, S.L. 1987. Projeto Irecê-Lapão. Salvador, CBPM (Convênio SME-CBPM). 
NOFFKE, N.; GERDES, G.; KLENKE, T.; KRUMBEIN, W.E. Microbially induced sedimentary structures - a new category within the classification of primary sedimentary structures. Journal of Sedimentary Research, v. 71, n. 5, p. 649-656, 2001.

OLIVEIRA, A.I.; LEONARDOS, O.H. Geologia do Brasil. Com. Bras. 1943.

PEDREIRA, A. J. O Supergrupo Espinhaço na Chapada Diamantina centro-oriental, Bahia: Sedimentologia, Estratigrafia e Tectônica. 1994. 126f. Tese (Doutorado em Geociências) Instituto de Geociências, Curso de Pós-Graduação em Geociências, Universidade de São Paulo, São Paulo, 1994.

PEDREIRA, A. J. et al. Projeto Bacia do Irecê II: relatorio final. Texto. Salvador: CPRM, 1987. v. 1. $168 \mathrm{p}$.

POSAMENTIER, H.W.; VAIL, P.R. Eustatic controls on clastic deposition sequence and systems tract models. Special publication SEPM, p.125-154, 1988.

RAJA GABAGLIA, G.P. Cyclostratigraphy and climatic control on microbiol influenced sedimentation (Balbuena Supersequence, Yacoraite Formation, Paleocene, Salta Argentina). IAS Meeting of Sedimentology, v. 28, p. 51, 2011.

READING, H. G. Sedimentary Environments: Process, Facies and Stratigraphy. Blackwell Science, 1996, $688 \mathrm{p}$. 
RENGER, F. A geologia do pré-cambriano do Norte da Bahia - Oeste de Sergipe. Debates sobre temas escolhidos. In: I SIMPÓSIO DO PRÉ-CAMBRIANO DO CRATON SANFRANCISCANO E DA PARTE NORTE-ORIENTAL DO BRASIL, 1, Aracaju, 1973, Anais Aracaju, Soc. Bras. Geol., 1973. v. 2, p. 152.

REIS, H.L.S.; GOMES, C.J.S.; FRAGOSO, D.G.C.; KUCHENBECKER, M. O cinturão epidérmico de antepaís da Bacia de Irecê, Cráton do São Francisco: principais elementos estruturais e modelagem física analógica. Geologia USP, Sér. cient., v. 13, p. 112-139, 2013.

RIDING, R. Structure and composition of organic reefs and carbonate mud mounds: concepts and categories. Earth-Science Reviews, v. 58, p. 163-231, 2002.

RIDING, R. Microbial carbonates: the geological record of calcified bacterial-algal mats and biofilms. Sedimentology, v. 47, supplement 1, p. 179-214, 2000.

RIMANN, E. A Kimberlita no Brasil. Anais da Escola de Minas, Ouro Preto, v. 15, p. 27-32, 1917.

SANTOS, M.N.; CHEMALE JR., F.; DUSSIN, I.A.; MARTINS, M.; ASSIS, T.A.R.; JELINEK, A.R.; GUADAGNIN, F.; ARMSTRONG, R. Sedimentological and paleoenvironmental constraints of the Statherian and Stenian Espinhaço rift system, Brazil. Sedimentary Geology. v p. 47-59, 2013. 
SANTOS, T.C.; PIMENTEL, M.M.; BRITO NEVES, B.B., 2011. Proveniência de Sedimentos Detríticos da Porção Nordeste do Grupo Una/Bambuí, Chapada Diamantina-BA: Dados U-Pb SHRIMP, in: XIII Congresso Brasileiro de Geoquímica. p. 1263-1266.

SANTOS-PINTO, J.J.; PEUCAT, H.M.; BARBOSA, J.S.F.; FANNING, J.L. PAQUETTE. Crustal evolution between 2.0 and $3.5 \mathrm{Ga}$ in the southern Gavião block (Umburanas-BrumadoAracatu region), São Francisco Craton, Brazil: a 3.5-3.8 Ga proto-crust in the Gavião block? Journal of South American Earth Sciences, v. 40, p. 129-142, 2012.

SATO, K. et al. Microssonda lônica de Alta Resolução e de Alta Sensibilidade (SHRIMP Ile/MC) do Instituto de Geociências da Universidade de São Paulo, Brasil: método analítico e primeiros resultados. Geol. USP, Sér. cient. , v. 14, n. 3, p. 3-18. 2014.

SCHERER, C.M.S et al. Análise da arquitetura de fácies, geometria e heterogeneidades de sistemas siliciclásticos: o exemplo das Formações Guiné (Açuruá) e Tombador (Proterozoico), Chapada Diamantina. Relatório final de pesquisa. 2013.

SCHERER, C.M.S; SANTANA, A.V.A (Coord.). (2014). Análise estratigráfica de alta resolução em sistemas carbonáticos microbiais do Neoproterozoico - exemplo da Formação Salitre, BA. Relatório final de pesquisa. 252 p.

SCHOBBENHAUS, C.; KAUL, P.F.T. Contribuição à estratigrafia da Chapada Diamantina, Bahia Central. Mineração e Metalurgia, v. 53, n. 315, p. 116-120, 1971. 
SCHOBBENHAUS, C.; HOPPE, A.; BAUMMANN, A.; LORK, A. Idade U/Pb do vulcanismo Rio dos Remédios Chapada Diamantina, Bahia. In: 380 Congresso Brasileiro de Geologia, Resumos Expandidos. Sociedade Brasileira de Geologia, p. 397-398. 1994.

SCHOLLE, P.A.; ARTHUR, M.A.; EKDALE, A.A.; Pelagic environment. In: SCHOLLE, P.A.; BEBOUT, D.G.; MOORE, C.H (Eds.), Carbonate Depositional Environments, American Association of Petroleum Geologists Memoir 33, 1983, p. 619-691.

SCHOLLE, P.A.; ULMER-SCHOLLE, D.S.A. Color guide to the petrography of carbonate rocks: Grains, textures, porosity, diagenesis. AAPG Memoir 77, 2003, 486 p.

SOUZA, S.L.; BRITO, R.W.S.; SILVA, P.C.R. Estratigrafia, Sedimentologia e Recursos Minerais da Formação Salitre na Bacia de Irecê, Bahia. Salvador, Companhia Baiana de Pesquisa Mineral (CBPM), 36 p, 1993. (Série Arquivos Abertos 2).

SRIVASTAVA, N.K. 1982. Algumas Observações sobre os Estromatólitos dos Grupos Una (Bahia) e Vaza Barrís (Sergipe), Nordeste do Brasil. Ciências da Terra, 3, p. 7-11.

SRIVASTAVA, N.K. 1986. Os Estromatólitos do Projeto Bacia de Irecê II. Natal:s./s.n./.9 p. Trabalho de consultoria para CPRM. Inédito.

STRASSER, A.; PITTET, B.; HILLGÄRTNER, H.; PASQUIER, J.-B., 1999. Depositional sequences in shallow carbonate-dominated sedimentary systems: concepts for a high-resolution analysis. Sedimentary Geology 128, 201-221. 
TERRA, G.J.S.; SPADINI, A.R.; FRANÇA, A.B.; SOMBRA, C.L.; ZAMBONATO, E.E.; JUSCHAKS, L.C.S.;... WINTER, W.R. Classificação de rochas carbonáticas aplicável às bacias sedimentares brasileiras. Boletim de Geociências Petrobras, v. 18, n. 1, p. 9-29, 2010.

TORQUATO, J.R.F.; MISI, A. Medidas isotópicas de carbono e oxigênio em carbonatos do Grupo Bambuí a região Centro-Norte do Estado da Bahia: Rev. Bras. Geoc., v. 7, p. 14-24, 1977.

TOULKERIDIS T., BABINSKI M., BUCHWALDT R., BRITO NEVES B.B., TODT W. \& SANTOS, R. 1999. Are varangian or sturtian the glacial deposits on the São Francisco Cráton? Evidence from determination of sedimentary rocks and minerals of the Neoproterozoic Una Group. In: South American Symposium on Isotope Geology, 2, Cordoba, Anais, p. 453-456.

TUCKER, M.E.; WRIGHT, V.P. Carbonate Sedimentology. Oxford: Blackwell Science, 1990. $482 \mathrm{p}$.

TUCKER, M.E.; WRIGHT, V.P. Carbonate Sedimentology. 2.ed. Oxford: Blackweel Scientific, 2008.

ULBRICH, H.H.G.J.; ULBRICH, M.N.C.; FERREIRA, F.J.F.; ALVES, L.S.; GUIMARÃES, G.B.;FRUCHTING,A.Levantamentos gamaespectrométricos em Granitos Diferenciados. In: Revisão da Metodologia e do Comportamento Geoquímico dos Elementos K, Th e U. Revista Geologia USP Série Científica, v. 9, n. 1, p. 33-53, 2009. 
VAIL, P.R.; MITACHUM JUNIOR, R.M.; TODD, R.G., et al. Seismic stratigrapy and global changes of sea level. In: PAYTON, C. E. (Org.) Seismic Stratigaphy Applications to Hydrocarbon Exploration. Tulsa: AAPG, 1977. Memoir, 26. p. 49-212.

VIEIRA, L.C.; TRINDADE, R.F.; NOGUEIRA, A.C.R.; ADER, M. Identification of a Sturtian cap carbonate in the Neoproterozoic Sete Lagoas carbonate platform, Bambuí Group, Brazil. CR Geosciences, v. 339, p. 240-258, 2007.

WALKER, R.G.; JAMES, N.P. (Ed.) Facies Models: Response to sea level change. Toronto: Geological Association of Canada, 1992. 409 p.

WALTER, M.R. Stromatolites. Amsterdam, Elsevier Scientific Publishing Company, 1976. 790 p.

WENTWORTH, C.K. A scale of grade and class terms for clastic sediments. The Journal of Geology, Chicago, v. 30, n. 5, p. 377-392, 1922.

WILLIAMS, H.E. Estudos geológicos na Chapada Diamantina. Rio de Janeiro, Serviço Geológico e Mineralógico do Brasil, 1930.

WILLIAMS, I.S. U-Th-Pb geochronology by ion microprobe. In: McKibben, M.A.; Shanks III, W.C.; Ridley, W.I.; (Ed.), "Applications of microanalytical techniques to understanding 
mineralizing processes", Reviews in Economic Geology Special Publication, v. 7, p. 1-35, 1998.

WIZEVICH, M.C. Photomosaics of outcrops: useful photographic techniques. In: MIALL, A.D.; Tyler, N. (Ed.), The three-dimensional facies architecture of terrigenous clastic sediments, and its implications for hydrocarbon discovery and recovery. Tulsa: SEMP Geology, 1991. p. 22-24. 
APÊNDICES 


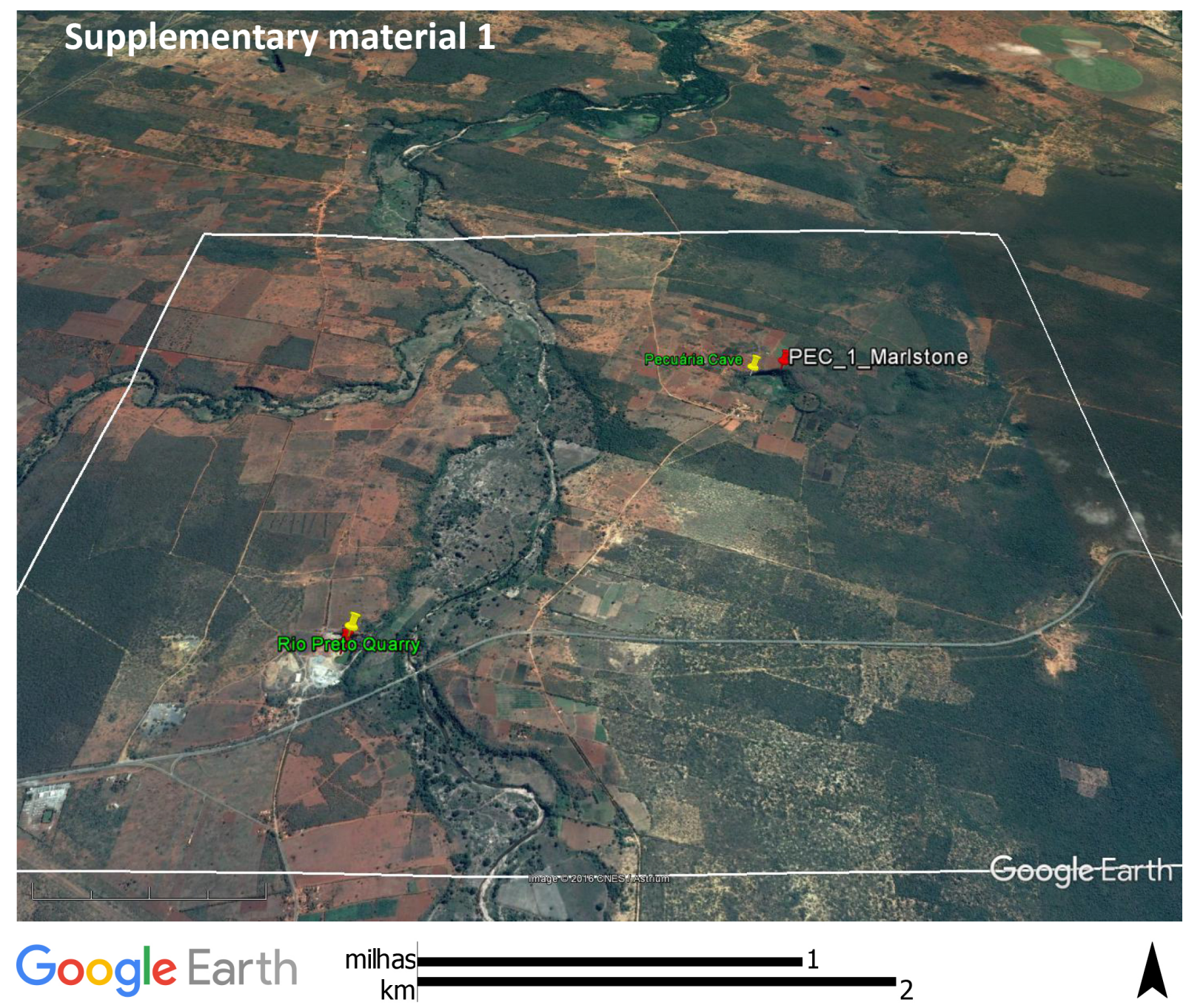

Supplementary material 1

Geospatial data . kml of the study area - VER CAPÍTULO 5 
Supplementary material 2 - Analytical data -VER CAPÍTULO 5

ACME ANALYTICAL LABORATORIES LTD.

Job Number:

GO112001722

Number of Samples:

Final Report

\begin{tabular}{|c|c|c|c|c|c|c|c|c|c|c|c|c|c|c|c|c|c|c|c|c|}
\hline & Method & WGHT & $4 x$ & $4 X$ & $4 x$ & $4 X$ & $4 x$ & $4 \mathrm{x}$ & $4 x$ & $4 x$ & $4 x$ & $4 x$ & $4 x$ & $4 x$ & $4 x$ & $4 x$ & 2A Leco & 2A Leco & $4 \mathrm{~B}$ & $4 B$ \\
\hline & Analyte & Wgt & $\mathrm{Al} 2 \mathrm{O} 3$ & $\mathrm{Ba}$ & $\mathrm{CaO}$ & $\mathrm{Cr} 2 \mathrm{O} 3$ & $\mathrm{Fe} 2 \mathrm{O} 3$ & K2O & LOI & MgO & $\mathrm{MnO}$ & $\mathrm{Na} 2 \mathrm{O}$ & P2O5 & $\mathrm{SiO} 2$ & SUM & TiO2 & TOT/C & TOT/S & $\mathrm{Rb}$ & $\mathrm{Sr}$ \\
\hline & Unit & KG & $\%$ & $\%$ & $\%$ & $\%$ & $\%$ & $\%$ & $\%$ & $\%$ & $\%$ & $\%$ & $\%$ & $\%$ & $\%$ & $\%$ & $\%$ & $\%$ & PPM & PPM \\
\hline & MDL & 0.01 & 0.01 & 0.01 & 0.01 & 0.001 & 0.01 & 0.01 & -5.11 & 0.01 & 0.01 & 0.01 & 0.01 & 0.1 & 0.01 & 0.01 & 0.02 & 0.02 & 0.1 & 0.5 \\
\hline Sample & Type & & & & & & & & & & & & & & & & & & & \\
\hline Sampie & Type & & & & & & & & & & & & & & & & & & & \\
\hline QUARTZ_GO & Prep Blank & & $<0.01$ & $<0.01$ & 0.03 & 0.005 & 0.76 & $<0.01$ & 0.02 & $<0.01$ & 0.01 & $<0.01$ & $<0.01$ & 98.8 & 99.56 & 0.03 & $<0.02$ & $<0.02$ & 0.3 & 2.4 \\
\hline QUARTZ_GO & Prep Blank & & $<0.01$ & $<0.01$ & 0.01 & 0.004 & 0.70 & $<0.01$ & -0.03 & $<0.01$ & $<0.01$ & $<0.01$ & $<0.01$ & 99.2 & 99.88 & 0.03 & $<0.02$ & 0.03 & 0.3 & 1.5 \\
\hline PRP-03 & Rock & 0.04 & 2.66 & 0.04 & 42.61 & 0.001 & 1.11 & 1.14 & 34.55 & 2.21 & 0.11 & 0.16 & 0.07 & 14.7 & 99.51 & 0.13 & 11.26 & 0.26 & 26.8 & 294.5 \\
\hline PRP-04 & Rock & 0.02 & 3.43 & 0.03 & 39.30 & 0.005 & 1.46 & 1.08 & 34.19 & 3.98 & 0.09 & 0.23 & 0.07 & 15.7 & 99.69 & 0.16 & 10.80 & 0.06 & 30.7 & 267.6 \\
\hline PRP-05 & Rock & 0.03 & 5.72 & 0.06 & 29.56 & 0.005 & 2.21 & 2.20 & 28.93 & 6.53 & 0.08 & 0.30 & 0.12 & 23.4 & 99.42 & 0.28 & 8.64 & 0.04 & 57.2 & 212.8 \\
\hline PRP-06 & Rock & 0.02 & 2.69 & 0.02 & 37.48 & 0.004 & 1.32 & 0.75 & 33.49 & 4.48 & 0.07 & 0.17 & 0.06 & 19.2 & 99.85 & 0.14 & 10.65 & $<0.02$ & 23.2 & 262.9 \\
\hline PRP-07 & Rock & 0.02 & 2.47 & 0.02 & 40.83 & 0.003 & 1.20 & 0.76 & 36.41 & 4.88 & 0.07 & 0.12 & 0.05 & 13.4 & 100.36 & 0.13 & 11.84 & 0.08 & 25.2 & 256.6 \\
\hline PRP-08 & Rock & 0.04 & 5.73 & 0.05 & 32.37 & 0.005 & 1.81 & 2.37 & 28.34 & 4.17 & 0.06 & 0.34 & 0.10 & 25.0 & 100.64 & 0.28 & 8.54 & $<0.02$ & 56.3 & 210.2 \\
\hline PRP-09 & Rock & 0.05 & 3.95 & 0.04 & 39.63 & 0.001 & 1.27 & 1.54 & 33.18 & 3.06 & 0.06 & 0.29 & 0.07 & 16.7 & 100.00 & 0.20 & 10.56 & 0.12 & 37.8 & 265.6 \\
\hline PRP-10 & Rock & 0.07 & 3.60 & 0.04 & 34.13 & 0.003 & 1.37 & 1.86 & 33.71 & 6.90 & 0.11 & 0.08 & 0.07 & 17.7 & 99.72 & 0.18 & 10.67 & 0.02 & 45.6 & 230.9 \\
\hline PRP-11 & Rock & 0.01 & 6.02 & 0.05 & 30.63 & 0.006 & 2.02 & 2.20 & 27.85 & 5.10 & 0.06 & 0.52 & 0.11 & 24.8 & 99.65 & 0.29 & 8.59 & $<0.02$ & 52.8 & 217.7 \\
\hline PRP-12 & Rock & 0.01 & 4.27 & 0.03 & 34.94 & 0.004 & 1.93 & 1.24 & 32.95 & 6.33 & 0.07 & 0.39 & 0.08 & 17.8 & 100.21 & 0.21 & 10.07 & 0.02 & 33.7 & 222.3 \\
\hline PRP-13 & Rock & 0.08 & 2.88 & 0.03 & 42.82 & 0.004 & 0.94 & 1.09 & 35.26 & 2.49 & 0.07 & 0.29 & 0.06 & 14.6 & 100.61 & 0.13 & 10.99 & 0.03 & 24.2 & 243.1 \\
\hline PRP-14 & Rock & 0.23 & 3.92 & 0.02 & 34.23 & 0.003 & 1.82 & 1.19 & 33.46 & 7.07 & 0.08 & 0.22 & 0.08 & 18.0 & 100.23 & 0.19 & 10.60 & $<0.02$ & 30.5 & 197.2 \\
\hline PRP-15 & Rock & 0.12 & 1.05 & $<0.01$ & 46.70 & 0.001 & 0.58 & 0.42 & 38.02 & 1.54 & 0.06 & 0.04 & 0.03 & 11.6 & 100.12 & 0.05 & 12.20 & 0.03 & 10.4 & 348.7 \\
\hline PRP-16 & Rock & 0.08 & 12.76 & 0.13 & 8.77 & 0.012 & 4.44 & 6.14 & 11.10 & 4.42 & 0.03 & 0.11 & 0.53 & 50.2 & 99.24 & 0.63 & n.a. & $<0.02$ & 134.0 & 52.1 \\
\hline PRP-19 & Rock & 0.05 & 4.46 & 0.04 & 33.11 & 0.004 & 1.51 & 1.92 & 33.01 & 8.05 & 0.11 & 0.08 & 0.12 & 17.7 & 100.30 & 0.21 & 10.20 & 0.02 & 41.6 & 209.0 \\
\hline
\end{tabular}

FLUORESCENCE LABORATORY I UNIVERSITY OF BRASILIA

Number of Samples:

\begin{tabular}{|c|c|c|c|c|c|c|c|c|c|c|c|c|c|c|c|c|c|c|c|c|}
\hline Method & X-Ray & icence & nnique & & & & & & & & & & & & & & & & & \\
\hline Analyte & & & $\mathrm{Al} 2 \mathrm{O} 3$ & $\mathrm{Ba}$ & $\mathrm{CaO}$ & $\mathrm{Cr} 2 \mathrm{O}$ & $\mathrm{Fe} 2 \mathrm{O} 3$ & K2O & LOI & $\mathrm{MgO}$ & $\mathrm{MnO}$ & $\mathrm{Na} 2 \mathrm{O}$ & P2O5 & $\mathrm{SiO} 2$ & SUM & TiO2 & TOT/C & TOT/S & $\mathrm{Rb}$ & $\mathrm{Sr}$ \\
\hline Unit & & KG & $\%$ & $\%$ & $\%$ & $\%$ & $\%$ & $\%$ & $\%$ & $\%$ & $\%$ & $\%$ & $\%$ & $\%$ & $\%$ & $\%$ & $\%$ & $\%$ & PPM & PPM \\
\hline PRP-21 & Rock & 0.01 & 1.96 & n.a. & 38.98 & n.a. & 0.76 & 0.51 & 40.38 & 5.96 & 0.02 & 0.23 & 0.05 & 10.8 & 100 & 0.1 & n.a. & n.a. & 1000 & 300 \\
\hline PRP-22 & Rock & 0.01 & 2.04 & n.a. & 44.49 & n.a. & 0.99 & 0.56 & 37.4 & 3.9 & 0.09 & 0.06 & 0.06 & 10.07 & 100 & 0.1 & n.a. & n.a. & 900 & 400 \\
\hline PRP-23 & Rock & 0.01 & 2.83 & n.a. & 36.88 & n.a. & 1.12 & 0.91 & 33.85 & 10.61 & 0.03 & 0.24 & 0.07 & 13.04 & 100 & 0.15 & n.a. & n.a. & 900 & 600 \\
\hline
\end{tabular}


Supplementary material 3 - (1) Comparison of tuffite composition with composition of the Upper Continental Crust (Taylor and McLennan, 1985) and Proterozoic volcanics and sedimentary rocks (Condie, 1993).

\section{MAJOR ELEMENTS}

\begin{tabular}{|c|c|c|c|c|c|c|c|c|}
\hline Sample & $\mathrm{SiO}_{2}$ & $\mathrm{Al}_{2} \mathrm{O}_{3}$ & $\mathrm{Fe}_{2} \mathrm{O}_{3} \mathrm{t}$ & $\mathrm{MgO}$ & $\mathrm{CaO}$ & $\mathrm{Na}_{2} \mathrm{O}$ & $\mathrm{K}_{2} \mathrm{O}$ & $\mathrm{TiO}_{2}$ \\
\hline TUFFITE OF SALITRE FM. (This study) - Sample PRP-16 & 50.20 & 12.76 & 4.44 & 4.42 & 8.77 & 0.11 & 6.14 & 0.63 \\
\hline Graywackes (Condie, 1993) & 66.10 & 15.00 & 5.80 & 2.10 & 2.60 & 2.80 & 2.50 & 0.77 \\
\hline Shale (Condie, 1993) & 63.10 & 17.50 & 5.65 & 2.20 & 0.71 & 1.06 & 3.62 & 0.64 \\
\hline Cratonic sandstone (Condie, 1993) & 92.15 & 3.87 & 1.32 & 0.55 & 0.45 & 0.51 & 0.88 & 0.17 \\
\hline Felsic volcanic rocks (Condie, 1993) & 73.30 & 13.20 & 2.70 & 0.50 & 1.20 & 3.20 & 4.00 & 0.25 \\
\hline Granite (Condie, 1993) & 73.30 & 13.50 & 2.30 & 0.42 & 1.30 & 3.20 & 4.80 & 0.28 \\
\hline UCC (Taylor and McLennan, 1985) & 66.00 & 15.20 & 5.00 & 2.20 & 4.20 & 3.90 & 3.40 & 0.50 \\
\hline
\end{tabular}

Notes: Large-ion lithophile, or LILE; High Field Strength Elements, or HFSE.

Eu/Eu* calculated according to Taylor \& McLennan (1985). 
Supplementary material 3 - cont. - (2) Comparison of tuffite composition with composition of the Upper Continental Crust (Taylor and McLennan, 1985) and Proterozoic volcanics and sedimentary rocks (Condie, 1993).

\begin{tabular}{|c|c|c|c|c|c|c|c|c|c|c|c|c|c|c|}
\hline \multirow[b]{3}{*}{ Sample } & \multicolumn{14}{|c|}{ TRACEELEMENTS } \\
\hline & \multicolumn{3}{|c|}{ LILE } & \multirow[b]{2}{*}{ Sc } & \multirow[b]{2}{*}{$v$} & \multirow[b]{2}{*}{ Co } & \multirow[b]{2}{*}{ Y } & \multicolumn{7}{|c|}{ HFSE } \\
\hline & $\mathrm{Ba}$ & $R b$ & $\mathrm{Sr}$ & & & & & $\mathrm{Zr}$ & $H f$ & $\mathrm{Nb}$ & $\mathrm{Ta}$ & $Y b$ & Th & $u$ \\
\hline TUFFITE OF SALITRE FM. (This study) - Sam ple PRP-16 & 1398 & 134 & 0.2 & 12 & 95 & 9.2 & & 151.6 & 4 & 12.7 & 0.8 & & 13.4 & 3.1 \\
\hline Grayw ackes (Condie, 1993) & 600 & 80 & 240 & 17 & 140 & 20 & 27 & 148 & 4.2 & 10 & 0.8 & 2.2 & 9 & 1.7 \\
\hline Shale (Condie, 1993) & 642 & 165 & 108 & 17 & 100 & 18 & 35 & 196 & 5.2 & 16.8 & 1.4 & 2.86 & 14.3 & 3.4 \\
\hline Cratonic sandstone (Condie, 1993) & 190 & 30 & 27 & 2.4 & 29 & 2.8 & 10.3 & 89 & 2.5 & 3.7 & 0.2 & 0.84 & 4.2 & 1.2 \\
\hline Andesite (Condie, 1993) & 648 & 35 & 315 & 23 & 154 & 26 & 22 & 145 & 4 & 8 & 0.5 & 2 & 3.4 & 1.8 \\
\hline Felsic volcanic rocks (Condie, 1993) & 952 & 125 & 150 & 15 & 48 & 5 & 35 & 230 & 6.5 & 15 & 1.2 & 2.6 & 8.2 & 2.5 \\
\hline Granite (Condie, 1993) & 750 & 156 & 120 & 5 & 20 & 5.5 & 45 & 240 & 7 & 20 & 1.5 & 3.5 & 18 & 4.5 \\
\hline UCC (Taylor and McLennan, 1985) & 550 & 112 & 350 & 11 & 60 & 10 & 22 & 190 & 5.8 & 25 & 2.2 & 2.2 & 10.7 & 2.8 \\
\hline
\end{tabular}

Notes: Large-ion lithophile, or LILE; High Field Strength Elements, or HFSE

Eu/EU* calculated according to Taylor \& McLennan (1985). 
Supplementary material 3 - cont. - (3) Comparison of tuffite composition with composition of the Upper Continental Crust (Taylor and McLennan, 1985) and Proterozoic volcanics and sedimentary rocks (Condie, 1993).

\begin{tabular}{|c|c|c|c|c|c|c|c|c|c|c|c|c|c|c|c|c|c|c|c|}
\hline Supplementary material 3 & & & & & & & & & & & & & & & & & & & \\
\hline & \multicolumn{14}{|c|}{ RARE EARTH EE EMENTS } & \multicolumn{5}{|c|}{ OTHERS } \\
\hline Sample & La & $\mathrm{Ce}$ & Pr & Nd & $S m$ & $E u$ & $G d$ & $T b$ & Dy & Ho & Er & $T m$ & $Y b$ & $L u$ & $E u / E u^{*}$ & $L a / Y b$ & $(L a / Y b) n$ & $\sum R E E$ & LREE/HREE \\
\hline TUFFITE OF SALITREFM. (This study) - Sample PRP-16 & 34.50 & 69.30 & 8.18 & 29.60 & 5.70 & 1.03 & 4.74 & 0.65 & 3.33 & 0.66 & 1.85 & 0.26 & 1.73 & 0.25 & 0.61 & 19.94 & 13.48 & 161.78 & 10.93 \\
\hline Grayw ackes (Condie, 1993) & 28.00 & 60.00 & & 26.00 & 4.90 & 0.93 & 4.34 & 0.66 & & & & & 2.20 & 0.38 & 0.62 & 12.73 & 8.60 & 127.41 & 15.69 \\
\hline Shale (Condie, 1993) & 38.00 & 81.70 & & 37.50 & 6.68 & 1.32 & 5.60 & 0.90 & & & & & 2.86 & 0.48 & 0.66 & 13.29 & 8.98 & 175.04 & 16.65 \\
\hline Cratonic sandstone (Condie, 1993) & 10.10 & 21.60 & & 9.00 & 1.75 & 0.36 & 1.52 & 0.23 & & & & & 0.84 & 0.13 & 0.67 & 12.02 & 8.13 & 45.57 & 15.38 \\
\hline Andesite (Condie, 1993) & 20.00 & 46.00 & & 25.00 & 5.30 & 1.30 & 4.94 & 0.77 & & & & & 2.00 & 0.32 & 0.78 & 10.00 & 6.76 & 105.63 & 11.99 \\
\hline Felsic volcanic rocks (Condie, 1993) & 25.00 & 51.20 & & 19.00 & 4.00 & 1.10 & 4.26 & 0.71 & & & & & 2.60 & 0.40 & 0.81 & 9.62 & 6.50 & 108.27 & 12.45 \\
\hline Granite (Condie, 1993) & 48.00 & 115.00 & & 54.00 & 8.70 & 1.00 & 8.17 & 1.28 & & & & & 3.50 & 0.58 & 0.36 & 13.71 & 9.27 & 240.23 & 16.68 \\
\hline UCC (Taylor and MCLennan, 1985) & 30.00 & 64.00 & 7.10 & 26.00 & 4.50 & 0.88 & 3.80 & 0.64 & 3.50 & 0.80 & 2.30 & 0.33 & 2.20 & 0.32 & 0.65 & 13.64 & 9.21 & 146.37 & 9.47 \\
\hline
\end{tabular}

Notes: Large-ion lithophile, or LLLE; High Field Strength Eements, or HFSE

Eu/Eu* calculated according to Taylor \& McLennan (1985). 